\title{
Scale-down and parallel operation of a riboflavin production process with Bacillus subtilis
}

\author{
Bettina Knorr \\ Vollständiger Abdruck der von der Fakultät für Maschinenwesen \\ der Technischen Universität München zur Erlangung des akademischen Grades eines \\ Doktor-Ingenieurs \\ genehmigten Dissertation.
}

Vorsitzender: Univ.-Prof. Dr.-Ing. habil. Boris Lohmann

Prüfer der Dissertation:

1. Univ.-Prof. Dr.-Ing. Dirk Weuster-Botz

2. Univ.-Prof. Dr. med., Dr. rer. nat. Adelbert Bacher

Die Dissertation wurde am 13.09.2005 bei der Technischen Universität München eingereicht und durch die Fakultät für Maschinenwesen

am 25.10.2005 angenommen. 

Meinen Eltern

Heide und Volker 



\section{Danksagung}

Ich möchte mich an dieser Stelle bei all denjenigen bedanken, die auf verschiedene Art und Weise zum Zustandekommen der vorliegenden Arbeit mit beigetragen haben.

Mein besonderer Dank gilt Prof. Dr.-Ing. Dirk Weuster-Botz für die hervorragende Betreuung und dafür, dass er mir die Möglichkeit gegeben hat, für meine Promotion ein sehr anwendungsorientiertes Thema zu bearbeiten. Das Vertrauen und die Freiheit, die er mir dabei gewährt hat, zusammen mit seiner Präsenz, wenn Diskussionen über Engpässe notwendig wurden, haben mir ein optimales Arbeiten ermöglicht. Darüber hinaus habe ich auch die beratenden Gespräche bezüglich meiner weiteren Entwicklung sehr geschätzt.

Ein weiteres Dankeschön geht an Dr. Heiner Schlieker, Dr. Peter Hohmann und Dr. Michael Hans von DSM Nutritional Products für die bemerkenswert offenen und konstruktiven Diskussionen und das persönlich sehr angenehme Klima während unserer Zusammenarbeit und für die Bereitstellung der Bacillus-Stämme. Außerdem an Dr. Nigel Mouncey, Andrea Kreutzer, René Faller und Dr. Jan Weber.

Dank auch an Prof. Dr. Dr. Adelbert Bacher vom Lehrstuhl für Organische Chemie und Biochemie der Technischen Universität München für sein freundliches Entgegenkommen bei der Ausgabe von DMRL.

Meinen Kollegen innerhalb der Gruppe "Hochdurchsatz-Bioprozessentwicklung" Robert Puskeiler, Andreas Kusterer und Andrea Vester gebührt mein großer Dank für ihre immer wieder tatkräftige fachliche Unterstützung und ihre Diskussionsbereitschaft. Ein super Team - es wäre nicht dasselbe ohne Euch gewesen!

Auch Karoline Geipel, Annkathrin Solf, Stephanie Heilmann, Stefanie Rubenwolf, Andreas Knepper, Angelika Beh, Ulrike Maurer, Claudia Drexler und Selina Biehl möchte ich noch einmal ausdrücklich danken. Eure Diplom- und Semesterarbeiten, Bachelor's Theses oder Praktika im Umfeld dieser Promotion haben wesentlich zu den Ergebnissen in der hier vorliegenden Form beigetragen. 
Georg Kojro aus der elektrischen und Norbert Werth und Karl-Heinz Zacher aus der mechanischen Werkstatt sei ein herzlicher Dank für die immer reibungslose Zusammenarbeit gesagt.

Im administrativen Bereich hat mir Irma Einsle sehr vieles erleichtert. Dafür, aber vor allem auch für unseren persönlichen Austausch, ein ganz besonderer Dank.

Die großartige Atmosphäre am Lehrstuhl für Bioverfahrenstechnik mit meinen übrigen Kollegen Maya, Julia, Hendrik, Helge, Jan, Vera, Andi \& Andy, Armin, Markus, Stefan, Dr. Greiner, Dr. Blumenberg und Dr. Hekmat hat in hohem Maße zu meinem Spaß an der Arbeit beigetragen. Besonders verbunden fühle ich mich Robert, Franco, Holger und Andrea für die freundschaftliche Nähe und die Unterstützung, die über fachliche Belange hinausging, und für die exzellente Stimmung im "Frauenzimmer".

Zusätzlich habe ich in den letzten vier Jahren auch im privaten Bereich viel Unterstützung und Verständnis erfahren. Danke dafür an alle meine Freunde, especially to Kristen and Klaudyne for your constant care and moral support, as well as to Vicki along with an extra thank you for proof-reading.

Abschließend möchte ich mich an meine Familie wenden, die mich auf den Weg gebracht hat, offen und kritisch die Welt zu betrachten und meinem Antrieb zu folgen, mich immer wieder neuen Herausforderungen zu stellen. Euer Vertrauen in mich stellt den wichtigsten Rückhalt in meinem Leben dar. Meine Dankbarkeit dafür lässt sich nicht in Worte fassen. 


\section{Preface}

This doctoral thesis is the result of research work carried out during four years at the Lehrstuhl für Bioverfahrenstechnik, Technische Universität München, Germany, under the supervision of Prof. Dr.-Ing. Dirk Weuster-Botz, in cooperation with DSM Nutritional Products (formerly Roche Vitamins), Basel, Switzerland.

The thesis is composed of seven chapters. The first one introduces the reader briefly to the area of bioprocess development relevant for this project. The thesis motivation and objective are described in the second chapter. The third chapter provides the theoretical background, upon which the discussion of the findings is based. The fourth chapter outlines the results of this project, which are summarized in the fifth chapter together with recommendations for future work. References can be found in the sixth chapter, before the final chapter provides a listing of the used symbols and abbreviations and details the applied materials and methods. 



\section{Table of Contents}

1 INTRODUCTION ............................................................................. 1

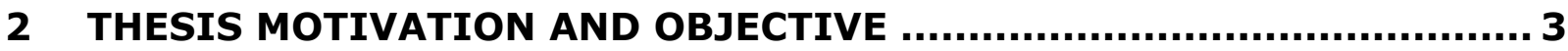

3 THEORETICAL BACKGROUND $\ldots \ldots \ldots \ldots \ldots \ldots \ldots \ldots \ldots \ldots \ldots \ldots \ldots \ldots \ldots \ldots \ldots \ldots \ldots \ldots \ldots$

3.1 Microbial production processes …......................................................... 7

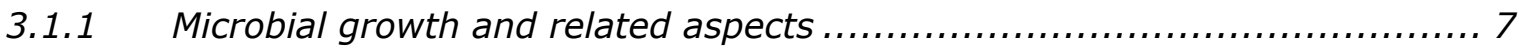

3.1.2 Oxygen transfer in aerated bioreactors .................................. 15

3.2 Scale-up and scale-down of aerobic bioprocesses ............................17

3.2.1 Approaches for scale-up of aerated bioreactors ........................... 17

3.2.2 Effects of substrate fluctuations in microbial cultivations..................... 19

3.3 Parallel experiments for bioprocess development ...........................21

3.3.1 Intention for the use of parallel experiments................................ 21

3.3.2 Classic and novel parallel screening systems..............................23

3.3.3 Automated setup with 48 stirred tank reactors at a milliliter scale ............25

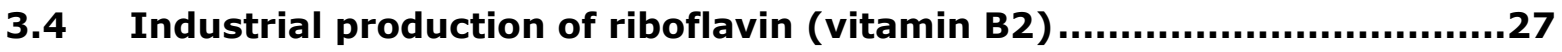

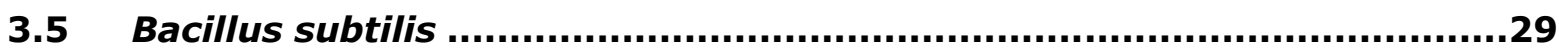

3.5.1 Central carbon metabolism in Bacillus subtilis .............................. 30

3.5.2 Riboflavin biosynthesis in Bacillus subtilis .................................. 31

3.5.3 Strain improvement of Bacillus subtilis.................................... 34 
4.1 Original B2 process at laboratory scale (reference) ............................. 37

4.2 Considerations for the transfer to the milliliter setup.......................... 48

$4.3 \quad$ Investigations at laboratory scale ................................................... 50

4.3.1 Effect of intermittent feeding during B2 processes ........................ 50

4.3.2 B2 processes at ambient pressure with oxygen addition ................... 57

4.3.3 B2 processes with intermittent feeding and $\mathrm{pH}$ control ......................60 60

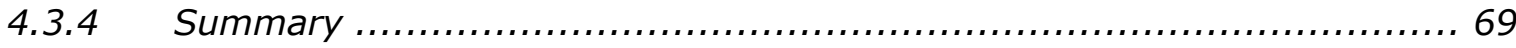

4.4 Parallel analyses for the automated milliliter process ........................ 71

4.4.1 Principle of the "all-in-one" analysis.................................... 71

4.4.2 Estimation of the dry cell mass ........................................ 72

4.4.3 Monitoring of the glucose concentration .................................. 74

4.4.4 Fluorimetric quantification of the riboflavin concentration................... 77

4.4.5 Summary .............................................................. 81

4.5 Automated parallel pH control at the milliliter scale........................... 83

4.5.1 Parallel pH measurement .............................................. 83

4.5.2 Resulting $\mathrm{pH}$ during $B 2$ processes in the milliliter setup ................... 87

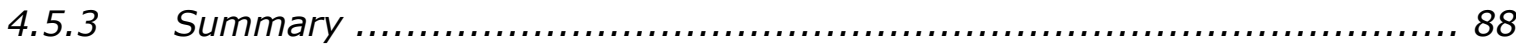

4.6 Automated parallel B2 processes at the milliliter scale ....................... 89

4.6.1 Scheduling the automated actions for 48 parallel B2 processes............. 89

4.6.2 Aseptic operation of the milliliter setup ................................. 91

4.6.3 Automated antifoam addition............................................. 94

4.6.4 Dissolved oxygen in the milliliter bioreactors............................. 95

4.6.5 Volumetric increase and evaporation in the milliliter setup ................. 100

4.6.6 Growth and productivity at the milliliter scale.............................. 104

4.6.7 Sequential and parallel reproducibility ................................... 110

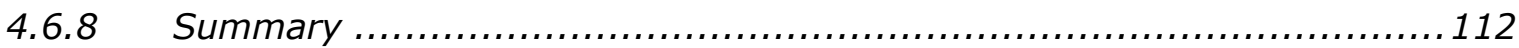

4.7 Guideline suggested for the scale-down of fed-batch processes......... 115 


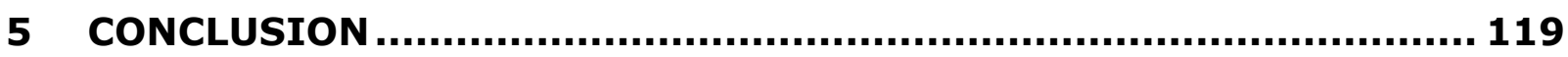

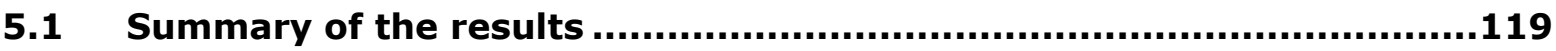

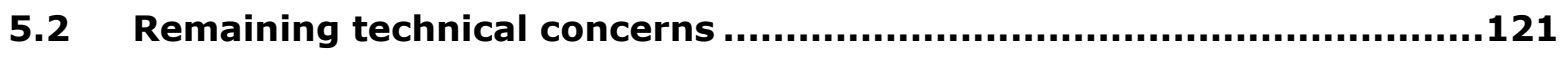

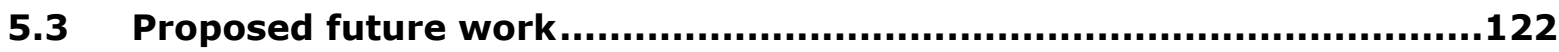

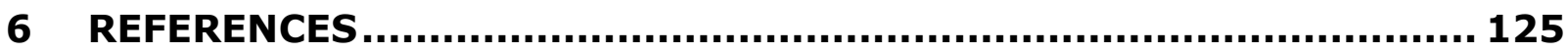

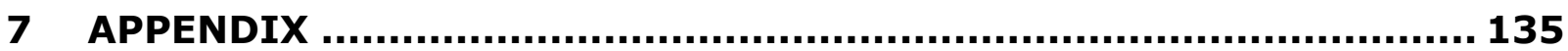

7.1 Abbreviations and symbols.......................................................... 135

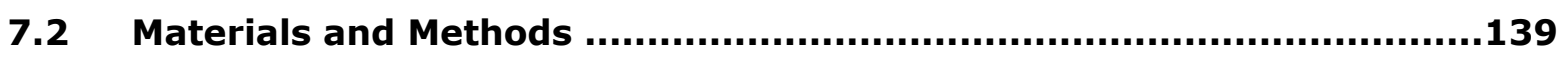

7.2.1 Equipment and Consumables........................................... 139

7.2.2 Solutions and media...................................................... 144

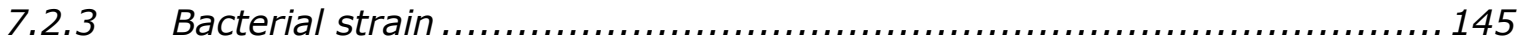

7.2.4 Preparation of cell stocks and seed cultures .............................. 145

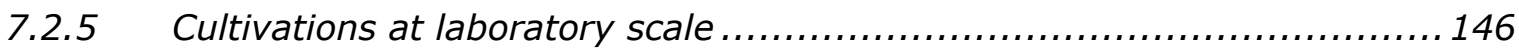

7.2.6 Cultivations in the automated milliliter setup .............................. 150

7.2.7 Gas input and measurement of dissolved oxygen in milliliter bioreactors. 152

7.2.8 Parallel pH measurement and control at the milliliter scale................... 154

7.2.9 Assessment of the dry cell mass concentration........................... 155

7.2.10 Quantification of riboflavin ............................................. 156

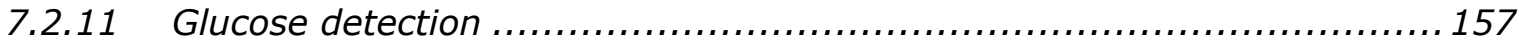

7.2.12 Liquid Handling by the laboratory robot................................... 159

7.2.13 Dilution scheme for the automated "all-in-one" analysis .................... 161

7.3 Additional information.

7.3.1 Settings of the plate reader for $p H, O D$, glucose, and riboflavin detection 162

7.3.2 IRIS scripts for intermittent operation at lab-scale....................... 164

7.3.3 Schematic outline of the 48 -fold schedule............................... 172

7.3.4 LabVIEW routines and GEMINI worklists integrated in GEMINI scripts..... 173

7.3.5 Liquid Classes .......................................................... 174 


\section{Introduction}

In the last two decades a growing number of biocatalytic production processes have been established in the pharmaceutical and fermentation industry either for new products or to substitute less effective chemical production processes. According to a recently updated report, the global market for fermentation products is expected to rise from US \$14.1 billion in 2004 at an average annual growth rate of $4.7 \%$ to US \$17.8 billion in 2009 (März, 2005). Today microorganisms as well as plant and animal cells are used for the manufacturing of enzymes, vitamins, aromatic compounds, antibiotics, proteins, antibodies, drug components and numerous other biochemical compounds.

Species of the genus Bacillus play an important role in industrial bioprocesses (Schallmey et al., 2004). They generate a variety of different products, such as the soya-based natto, numerous enzymes, insecticides, purine nucleotides, vitamin B2 (riboflavin), and the flavor agent ribose. The advantage of using Bacillus species for industrial purposes is that many of them are generally regarded as safe (GRAS) by the US Food and Drug Administration and they grow rather fast which allows short production cycles (Schallmey et al., 2004). In addition, the physiology and the genetics of Bacillus subtilis are well described, which allows directed metabolic engineering of the producer strains (Dauner and Sauer, 2001; Zamboni et al., 2003).

State of the art research activities often employ metabolic engineering and directed evolution for a constantly increasing output of new candidates, as promising production microorganisms or for further improved reactions (Chartrain et al., 2000; Hibbert et al., 2005). During the process development toward scale-up for industrial manufacture, the combination of different biocatalysts with various process strategies leads to matrix experiments with well over 100 single test runs (Freyer et al., 2004). Additionally, established production processes need to be revised regularly in order to remain competitive on the market. The large number of necessary screening experiments calls for devices suitable for highly parallel, preferably automated cultivations in order to save time and manpower (highthroughput bioprocess design). A miniaturization of the cultivations is particularly beneficial for reactions involving expensive media or substrates (Lye et al., 2003). 
Besides the most widely applied basic shaking flasks and improved instrumented variants thereof, the application of microtiter plates, as simple, highly parallel reactors for screening experiments, has gained importance (Weuster-Botz, 2005). Together with the advancing automation of the processing techniques at this scale (Lye et al., 2003), high throughput of test runs in these devices has become possible. However, the critical aspect in the evolution of automation at this scale is the ability to grow cultures in a manner reflective of large-scale bioreactors, which is where the productivity of the new strain or other attributes must ultimately have their impact (Parekh et al., 2000). Experiments aiming at a closer simulation of the actual process conditions in industrial stirred tank reactors are thus carried out in systems at a milliliter scale (Lamping et al., 2003; Puskeiler et al., 2004). These setups are intended to realize power input and oxygen transfer characteristics similar to the technical scale and provide a higher degree of monitoring and control capacity compared to the simple microtiter plate systems. 


\section{Thesis Motivation and Objective}

Stirred tank bioreactors at a milliliter scale, with magnetically driven gas-inducing impellers, have been developed during previous studies at the Lehrstuhl für Bioverfahrenstechnik (Technische Universität München) to enable high-throughput bioprocess design (Puskeiler et al., 2003, International Patent Application PCT EP03/14752; Puskeiler, 2004). These bioreactors are operated in a reaction block for up to 48 parallel vessels, together with a laboratory robot for automated dosing of additive solutions and for sample handling, and with a photometric plate reader for at-line analysis. Processes are controlled by the laboratory robot software which communicates with the other hardware components. This technique can be used for the screening of a relatively large number of parallel experiments under process conditions which are similar to laboratory or industrial scale conditions with respect to the generated oxygen transfer rates. Furthermore, the degree of process monitoring and control is enhanced compared to traditional shake flask cultures.

Cultivations with Escherichia coli have been carried out in batch or in fully automated intermittent fed-batch mode in the milliliter setup (5-10 mL) with automated compensation of $\mathrm{pH}$ changes and evaporation loss, and at-line monitoring of cell growth (Puskeiler et al., 2005a). The results have been reproduced in a standard stirred tank reactor at laboratory scale $(3 \mathrm{~L})$, mimicking the restricted conditions of the miniaturized system regarding the discontinuous addition of feed and base solution. However, it has never been attempted to replicate a microbial high performance production process, typically operated under optimized, unrestrained conditions in standard stirred tank reactors at a large scale, in the confined environment of the milliliter system. It is thus unknown, if the setup can be used for the screening of industrially relevant processes.

The primary goal of this thesis is to adapt an exemplary high performance process, operated in a fed-batch mode and with $\mathrm{pH}$ control, from a laboratory scale $(3 \mathrm{~L})$ to the automated 48 -fold miniaturized setup. The operational parameters that assure an equivalent process outcome, especially with respect to productivity and growth, shall be identified. This may allow the transfer of the results achieved in the milliliter setup to the laboratory or the technical scale. The production of 
riboflavin with Bacillus subtilis (B2 process) has been selected as a microbial production process with industrial relevance (DSM Nutritional Products, Switzerland) for the investigations during this thesis.

The milliliter technology available for this study is able to simulate process conditions in the miniaturized bioreactors remarkably similar to those found in conventional bench-top stirred tank bioreactors. However, certain attributes of the process operation at laboratory scale cannot be transferred to the automated screening setup. One example of this is the $\mathrm{pH}$ measurement which cannot be carried out online in the milliliter vessels. Another example is the liquid addition to the parallel reactors in this setup, which is conducted by the pipetting unit of the laboratory robot, resulting in the feed provision as well as the $\mathrm{pH}$ control not being continuous. Furthermore, the milliliter setup can only be operated at ambient pressure. Therefore, if excess pressure is applied at laboratory scale for increased oxygen uptake rates, this has to be compensated by oxygen addition to the gas flow in the milliliter setup.

The consequences of these operational modifications for the outcome of a high performance fed-batch production process are unknown. Generally the intention of using a fed-batch cultivation mode is to maintain a low growth rate of the employed microorganisms via a controlled provision of a growth-limiting substrate in a continuous nutrient flow. Thereby effects like substrate inhibition or overflow metabolism shall be prevented and the productivity shall be maximized. However, the use of the laboratory robot for the automated liquid addition in the milliliter setup and therefore the change from continuous feeding to an intermittent provision of nutrients, will result in dynamic shifts of the substrate concentrations. The peak concentrations may reach well above the critical values for substrate limitation and may provoke a change in the physiology of the used microorganisms, previously grown under limited conditions. Various physiological responses to substrate fluctuations have been reported for E. coli (Enfors et al., 2001; Lin and Neubauer, 2000; Weuster-Botz et al., 2001a). Due to these considerations, it is not clear, if the high performance of the originally continuously fed B2 process can be maintained during cultivations with dynamical changes due to an intermittent feeding mode. It is an objective of this thesis to investigate this matter.

In addition, analytical methods at a microliter scale shall be developed for fully automated, integrated at-line monitoring of the parallel B2 processes in the milliliter setup with respect to growth, substrate (glucose) consumption, and product (riboflavin) generation. 
Finally, a general guideline for the scale-down of other industrially or scientifically relevant fed-batch processes to the milliliter setup shall be established, based on the knowledge gained during the adaptation of the exemplary process.

For the iterative experimental scale-down of the B2 process to the automated milliliter system, the following tasks are recommended:

Laboratory scale (3 L)

- Establish the B2 process with Bacillus subtilis RB50::[pRF69] $]_{\mathrm{n}}$ at laboratory scale according to the original protocol from DSM Nutritional Products as a reference.

- Investigate the influence of intermittent substrate and base addition in pulses instead of continuous feeding and determine the optimal feeding interval for an unimpaired process performance.

- Investigate the impact of adding oxygen to the gas flow for sufficient oxygen supply to the process, instead of applying excess pressure.

Milliliter scale $(10 \mathrm{~mL})$ :

- Develop analytical procedures at a microliter scale suitable for an automated at-line determination of dry cell mass, glucose and riboflavin concentrations in the milliliter setup using a photometric reader for absorbance or fluorescence measurements in microtiter plates.

- Establish a parallel at-line $p H$ measurement and an intermittent $p H$ control, feasible for the use in the automated milliliter setup.

- Realize automated foam suppression in the milliliter setup.

- Develop a schedule (controlled by the laboratory robot) that allows the automated operation of 48 parallel riboflavin production processes at milliliter scale.

- Carry out automated parallel cultivations in the milliliter setup and assess the parallel and sequential reproducibility.

- Assess the occurrence of contaminations and develop strategies for avoiding contaminations or transfer of bacteria between different vessels in the milliliter setup via the robotic liquid handling. 


\section{Theoretical Background}

This chapter provides the theoretical background that forms the basis for the discussion of the results in this work. This includes some basic knowledge about biological cultivations (3.1) and general physical and physiological aspects that should be considered during the scale-up or scale-down of such processes (3.2). This is followed by the motivation for employing parallel experiments for process optimization, a comparison of currently available screening devices, and the description of the screening setup at milliliter scale which has been used during this project (3.3). The subsequent section briefly introduces the industrial production of riboflavin, focusing on the biotechnological production with Bacillus subtilis (3.4). The final part of this chapter describes the metabolism of this bacterium and its genetic modifications to yield a high-performance production strain (3.5).

\subsection{Microbial production processes}

\subsubsection{Microbial growth and related aspects}

The growth of heterotrophic microorganisms requires organic carbon compounds, for example glucose or glycerol, organic or inorganic nitrogen sources, mineral salts, and sometimes vitamins. These nutrients have to be provided with the growth media. An additional supply of a nitrogen source during microbial cultivations can be achieved by using ammonium hydroxide for $\mathrm{pH}$ adjustment. Oxygen is needed as a further substrate for growth of aerobic organisms.

Definition of specific rates regarding growth, substrate uptake and production

The specific growth rate $\mu$ is defined as the increase of the biomass concentration $c_{X}$ with time, divided by the actual biomass concentration (Equation 3-1).

Equation 3-1: $\quad \mu \equiv \frac{1}{c_{x}} \cdot \frac{d c_{x}}{d t}$

Analogous definitions render the specific substrate uptake rate $q_{s}$ (Equation $3-2$ ), the specific oxygen uptake rate $q_{0}$ (Equation 3-3), and the specific product forma- 
tion rate (Equation 3-4), using the change of the substrate concentration $c_{S}$, oxygen concentration $c_{O}$, or product concentration $c_{P}$, respectively.

Equation 3-2: $\quad q_{s} \equiv \frac{1}{c_{x}} \cdot \frac{d c_{s}}{d t}$

Equation 3-3: $\quad q_{0} \equiv \frac{1}{c_{x}} \cdot \frac{d c_{0}}{d t}$

Equation 3-4: $\quad q_{p} \equiv \frac{1}{c_{x}} \cdot \frac{d c_{p}}{d t}$

Formal kinetics and yield coefficients

The specific substrate uptake rate can be described as the sum of separate uptake rates, depending on the use of the substrate, for growth $q_{s, \mu}$, maintenance metabolism of the cells $q_{s, m}$, or product generation $q_{S, P}$ (Equation 3-5).

Equation 3-5: $\quad q_{S}=q_{S, \mu}+q_{S, m}+q_{S, P}$

The maintenance metabolism incorporates all the cellular processes necessary for essential metabolic functions such as the maintenance of an energized membrane, i.e. the proton-motive force, the repair of damage to cellular structures, and the motility of motile organisms (Shuler and Kargi, 1992). The substrate uptake rate for maintenance of a given system is often regarded as a constant, which is called the maintenance coefficient $m_{s}$.

According to formal kinetic model assumptions, the differential growth yield coefficients $Y_{x / S}^{*}$ and $Y_{x / O}^{*}$ on substrate or oxygen, respectively, and the differential product yield coefficient $Y_{P / S}^{*}$ are defined (Equation 3-6, Equation 3-7, and Equation 3-8).

Equation 3-6: $\quad Y^{*}{ }_{x / S} \equiv \frac{\mu}{q_{s, \mu}}=\frac{d c_{X} / d t}{d c_{s} / d t}=\frac{d c_{X}}{d c_{s}}$

Equation 3-7: $\quad Y^{*}{ }_{x / 0} \equiv \frac{\mu}{q_{0}}=\frac{d c_{x} / d t}{d c_{0} / d t}=\frac{d c_{x}}{d c_{0}}$

Equation 3-8: $\quad Y^{*}{ }_{P / S} \equiv \frac{q_{p}}{q_{S, P}}=\frac{d c_{p} / d t}{d c_{S} / d t}=\frac{d c_{p}}{d c_{S}}$ 
Typical values for the growth yield coefficient are $0.4-0.6 \mathrm{~g} \mathrm{~g}^{-1}$ for most bacteria growing aerobically on glucose, while the growth yield coefficient on oxygen is 0.9-1.4 $\mathrm{g} \mathrm{g}^{-1}$ (Shuler and Kargi, 1992).

Bringing the differential yield coefficients and the maintenance coefficient $m_{S}$ into Equation 3-5, the substrate uptake rate can be written as Equation 3-9.

Equation 3-9: $\quad q_{s}=\frac{\mu}{Y^{*}{ }_{X / S}}+m_{S}+\frac{q_{p}}{Y^{*}{ }_{P / S}}$

The differential yield coefficients are often afflicted with large errors due to the limited accuracy of measurement of the respective concentrations. Therefore, it is usually more practical to use integral yields for the characterization of microbial production processes. The integral yields are calculated based on the amount of biomass or product, generated during the period from the beginning of a process at $t_{0}$ until a certain time $t$, divided by the amount of substrate consumed during the same time (Equation 3-10 and Equation 3-11, respectively).

Equation 3-10: $\quad Y_{x / S}=-\frac{\left.\Delta c_{x}\right|_{t_{o}} ^{t}}{\left.\Delta c_{s}\right|_{t_{0}} ^{t}}$

Equation 3-11: $\quad Y_{P / S}=-\frac{\left.\Delta c_{P}\right|_{t_{0}} ^{t}}{\left.\Delta c_{S}\right|_{t_{0}} ^{t}}$

\section{Typical modes for the operation of bioprocesses}

There are essentially three different modes of operation for the cultivation of microorganisms, which are batch, fed-batch or continuous mode. During batch cultivations, all the necessary nutrients are contained in the initial growth medium, with the exception of oxygen, which is added continuously throughout the process via aeration, and possibly acid or base for the adjustment of the $\mathrm{pH}$.

A typical growth curve during batch cultivations includes the following phases: lag phase, acceleration phase, logarithmic or exponential growth phase, deceleration phase, stationary phase, and death phase, as depicted exemplarily in Figure 3-1. The lag phase occurs immediately after inoculation and is a period of adaptation of cells to the new environment. Growth is not observed before the beginning of the acceleration phase. At the end of this phase the cells reach the maximum growth rate, which is maintained during the subsequent exponential growth phase. The exponential growth phase is characterized by no growth limitation by any of the substrates. When one or several substrates become limiting, the cells 
enter the deceleration phase and the growth slows down. The stationary phase commences when the net growth rate is zero, which is either when there is no cell division or when the growth rate is equal to the death rate. In this phase, cells may still be metabolically active and produce secondary metabolites, which are not growth-related. In fact, the production of certain metabolites is enhanced during the stationary phase due to metabolite deregulation. In both the stationary and the subsequent death or decline phase, it is important to recognize that there is a distribution of properties among individuals in a population. In a narrow distribution, cell death will occur nearly simultaneously; in a broad distribution, however, a sub-fraction of the population may survive for an extended period (Shuler and Kargi 1992).

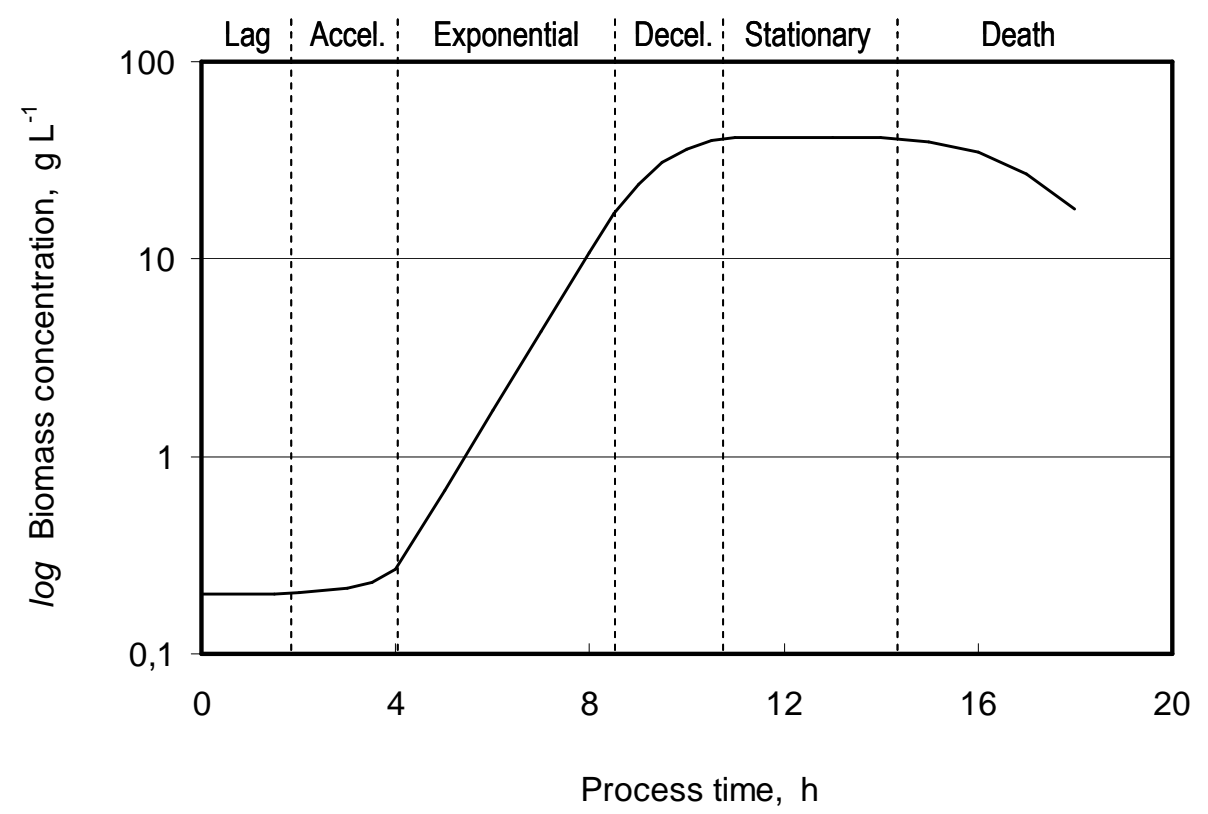

Figure 3-1: Typical batch growth for a bacterial population, divided into lag phase, acceleration phase (Accel.), exponential growth phase, deceleration phase (Decel.), stationary phase, and death phase.

The growth rate during the exponential growth phase and the deceleration phase can be described by a formal kinetic model according to Monod (1949) as a function of the concentration of one limiting substrate $c_{S}$. The simplest version of the model neglects maintenance metabolism and product generation (Equation 3-12) and assumes that further process variables like temperature and pressure remain constant.

Equation 3-12: $\quad \mu=\mu_{\max } \cdot \frac{c_{s}}{c_{s}+K_{s}}$ 
In Equation 3-12 $K_{S}$ is the saturation constant of the limiting substrate and $\mu_{\max }$ is the maximum specific growth rate, which is reached during the exponential growth phase when the substrate concentration is significantly higher than the saturation constant (unlimited growth). When the substrate concentration becomes equal to the saturation constant, the growth rate is only half of the maximum growth rate. The correlation is illustrated in Figure 3-2. It becomes approximately linear at very small substrate concentrations clearly below the saturation constant (reaction of $1^{\text {st }}$ order).

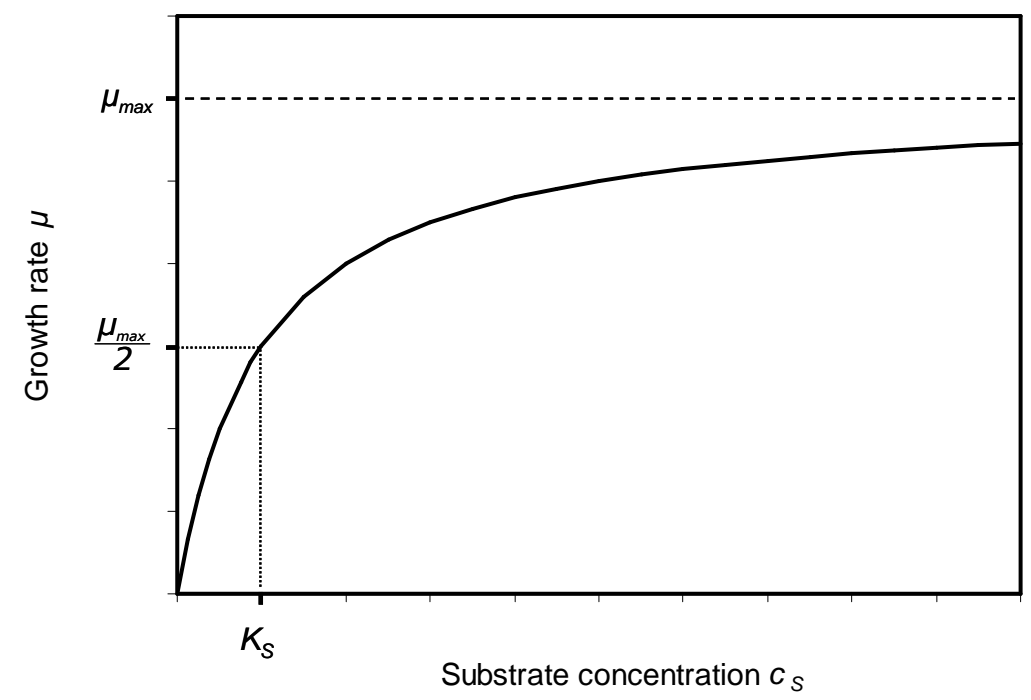

Figure 3-2: Growth rate dependence on the limiting substrate concentration according to the kinetic model of Monod (1949). $K_{S}$ designates the saturation constant of the limiting substrate.

A review of microbial growth kinetics which were assessed for different strains of the bacterium Escherichia coli, growing in batch cultures on glucose as the sole carbon source at $30-37^{\circ} \mathrm{C}$, reports saturation constants $K_{S}$ between 0.2 $2.3 \mathrm{mg} \mathrm{L}^{-1}$ and maximum specific growth rates $\mu_{\max }$ between $0.75-1.23 \mathrm{~h}^{-1}$ (Kovárová-Kovar and Egli, 1998).

During some processes the excess of a substrate can have an inhibitory effect on the cellular growth. Additionally, generated products or by-products may be inhibiting. It should be mentioned that these effects, as well as the maintenance metabolism and the influence of further limiting substrates, for example oxygen, can be integrated in the mathematical model of Monod.

When high substrate concentrations have no negative effects on the desired biological reactions, batch cultivations can be applied advantageously, particularly because of the low effort for process control. When substrate inhibition or overflow metabolism with a generation of undesired by-products occurs, the substrate 
concentration must be kept low for optimum growth or product formation. In this case, the fed-batch mode of operation is used.

In fed-batch cultivations, nutritional medium is continuously fed to the reactor, but no medium is removed, resulting in an increasing liquid volume with process time. Fed-batch operation allows the change from growth to metabolite overproduction by adapting the nutrient supply respectively, generally by keeping the substrate concentration in the vessel close to or below the saturation constant $K_{S}$. For this reason, a large number of industrial processes are carried out in fed-batch mode. Since this mode is characterized by very low growth rates at later stages, the relative importance of cellular maintenance processes increases. This aspect is thus of both scientific and industrial interest (Arbige et al., 1993).

The third mode of operation is the continuous cultivation, which is characterized by a permanent inflow of substrates to the reactor and outflow of medium including cells from the reactor, usually both with the same rate so that a steady state can be reached. According to the criterion controlling the flow rate, several types of continuous operation are obtained. The most important is the chemostat that is operated at a fixed inflow rate (Bellgardt, 2000). Investigations at steady state have become a valuable tool for the characterization of metabolic cellular stages independent of concentration gradients or dynamic variations. However, for industrial production continuous culture is not common, particularly not in the pharmaceutical industry. This is because the probability of contamination or mutation is higher compared to the other modes and the definition of a batch size is not evident (Thiry and Cingolani, 2002).

\section{Hydrogen ion concentration $(\mathrm{pH})$}

The $p H$ affects the activity of enzymes and therefore the microbial growth rate. The optimal $\mathrm{pH}$ for growth may differ from that for product formation. Generally, the acceptable $p H$ range varies about the optimum by \pm 1 to $2 p H$ units. During microbial cultivations, the $\mathrm{pH}$ can change dependent on the nitrogen source or because of the generation of organic acids, the utilization of acids, particularly amino acids, or the production of bases. In addition, the evolution of carbon dioxide can greatly influence the $\mathrm{pH}$ (Shuler and Kargi, 1992). Thus, $\mathrm{pH}$ control by means of a buffer or an active $\mathrm{pH}$ control system is important.

\section{Balances of the liquid phase in ideal bioreactor models}

The model assumption for ideal stirred tank reactors is that the concentrations $c_{i}$ and specific reaction rates $r_{i}$ of all components $i$, as well as the physical and chemical process parameters, such as pressure, temperature and $\mathrm{pH}$, are equal at 
any location in the vessel (isotropic reactor). Under these conditions, the mass balances for substrate, biomass and product concentration can be calculated according to Equation 3-13, in which $V_{R}$ is the reactor volume and $\dot{V}_{\text {in }}$ and $\dot{V}_{\text {out }}$ are the inflow and outflow rates, respectively.

Equation 3-13: $\quad \frac{d c_{i}}{d t}=\frac{\dot{V}_{\text {in }}}{V_{R}} \cdot c_{i, \text { in }}-\frac{\dot{V}_{\text {out }}}{V_{R}} \cdot c_{i}+c_{x} \cdot r_{i}$

During batch cultivations the inflow and outflow rates are zero; changes of concentration of the respective component are thus only the result of the reactions in the reactor. For continuous cultivations the balance equals zero (no change of concentrations with time). For fed-batch processes the mass balances for substrate uptake, cell growth and product formation are given by Equation 3-14, Equation 3-15 and Equation 3-16, respectively.

Equation 3-14: $\quad \frac{d c_{s}}{d t}=\frac{\dot{V}_{i n}}{V_{R}} \cdot\left(c_{s, i n}-c_{S}\right)-c_{x} \cdot q_{S}$

Equation 3-15: $\quad \frac{d c_{x}}{d t}=-\frac{\dot{V}_{\text {in }}}{V_{R}} \cdot c_{x}+c_{x} \cdot \mu$

Equation 3-16: $\quad \frac{d c_{p}}{d t}=-\frac{\dot{V}_{i n}}{V_{R}} \cdot c_{p}+c_{X} \cdot q_{P}$

Equation 3-15 can be simplified to Equation 3-17, when the dilution of the cell concentration by the inflow of feed solution during a certain period is small enough to be neglected.

Equation 3-17: $\quad \frac{d c x}{d t}=\mu \cdot c x$

Integration of this equation during the exponential growth phase of a culture renders Equation 3-18, if maintenance requirements are not considered.

Equation 3-18: $\quad c_{x}=c_{x O} \cdot e^{\mu_{\max } \cdot t} \quad\left(\right.$ or $\left.\quad \ln \frac{C_{x}}{C_{x O}}=\mu_{\max } \cdot t\right)$

in which $c_{x O}$ is the cell concentration at the beginning of the exponential growth phase at $t=0$.

Assuming a constant substrate concentration $c_{S}$ in the reactor, which is negligible compared to the substrate concentration in the inflowing nutrient solution $c_{S, i n}$ 
Equation 3-14, Equation 3-18 and Equation 3-9 can be used to calculate an exponential feed flow rate for a constant growth rate, if substrate uptake for product generation or maintenance metabolism are not considered (Equation 3-19).

Equation 3-19: $\quad \dot{V}_{\text {in }} \cong \frac{V_{R}}{c_{S, \text { in }}} \cdot c_{x 0} \cdot e^{\mu_{\text {max }} \cdot t} \cdot \frac{\mu}{Y^{*} x / s}$

A constant growth rate can only be maintained as long as no other substrate becomes limiting and none of the products inhibits the growth. However, during aerobic cultivations the oxygen supply often becomes a limiting factor, so that linear or constant feeding profiles are used during many applications rather than an exponential profile. In these cases, the growth rate declines continually with elevating process time.

\section{Balances of the gas phase}

Dissolved oxygen is an important substrate in aerobic cultivations and has to be supplied constantly via the process gas. The oxygen uptake rate OUR during a cultivation can be assessed via the oxygen balance of the process gas (Equation 3-20).

Equation 3-20: $\quad$ OUR $=\frac{\dot{V}_{g, \text { in }} \cdot \gamma_{0, \text { in }}-\dot{V}_{g, \text { out }} \cdot \gamma_{0, \text { out }}}{V_{R} \cdot V_{\text {mol }}}$

in which $\gamma_{0, \text { in }}$ and $\gamma_{0, \text { out }}$ are the molar oxygen fractions in the inlet and outlet gas flow $\dot{V}_{g, \text { in }}$ and $\dot{V}_{g, \text { out }}$, respectively, and $V_{m o l}$ is the molar volume of ideal gases $\left(=22.414 \mathrm{~mol} \mathrm{~L}^{-1}\right)$.

The major catabolic end product in most microbial cultivations is carbon dioxide $\left(\mathrm{CO}_{2}\right)$. A calculation analogous to Equation 3-20 allows the estimation of the carbon dioxide evolution rate CER during a process (Equation 3-21).

Equation 3-21: $\quad C E R=\frac{\dot{V}_{g, \text { out }} \cdot \gamma_{\mathrm{CO}_{2}, \text { out }}-\dot{V}_{g, \text { in }} \cdot \gamma_{\mathrm{CO}_{2} \text {, in }}}{V_{R} \cdot V_{\text {mol }}}$

Usually only the gas concentrations in the outlet gas flow are measured. The molar fraction of oxygen in the inlet gas flow is approximately $20.95 \%$ for aeration with air, while the inlet fraction of carbon dioxide in this case can be regarded as zero fairly accurately for most applications. If the inlet and outlet gas flow are considered approximately equal, the balances can be simplified to Equation 3-22 and Equation 3-23. 
Equation 3-22: $\quad$ OUR $=\frac{\dot{V}_{g}}{V_{R} \cdot V_{m o l}} \cdot\left(\gamma_{O, \text { in }}-\gamma_{O, \text { out }}\right)$

Equation 3-23: $\quad C E R=\frac{\dot{V}_{g}}{V_{R} \cdot V_{\text {mol }}} \cdot \gamma_{\mathrm{CO}_{2} \text {, out }}$

The ratio between the carbon dioxide evolution rate and the oxygen uptake rate is called the respiratory coefficient $R Q$ (Equation 3-24), which can indicate certain metabolic states of a microbial population during a cultivation.

Equation 3-24: $\quad R Q \equiv \frac{C E R}{O U R}=\frac{\gamma_{\mathrm{CO}_{2} \text { out }}}{\gamma_{0, \text { in }}-\gamma_{0, \text { out }}}$

Generally the consumption of dissolved oxygen during a process can be physically ("gas stripping"), via chemical reactions, or biologically via the growth of the cultivated microorganisms. During aerobic microbial production processes the role of the biological oxygen consumption is usually the most crucial, especially at high cell concentrations. When the oxygen consumption is exclusively caused by microbial growth, the oxygen uptake rate can be calculated by using the specific oxygen uptake rate $q_{0}$ and the growth yield coefficient on oxygen (Equation 3-7) for ideally mixed stirred tank reactors (Equation 3-25).

Equation 3-25: $\quad$ OUR $=c_{x} \cdot q_{O}=\mu \cdot \frac{c_{X}}{Y^{*}{ }_{x / O}}$

Besides the oxygen requirement for growth, additional oxygen may also be needed for product generation during some processes. If the oxygen uptake rate exceeds the rate of oxygen supply, this leads to oxygen limitation. Shuler and Kargi (1992) mention 40-200 $\mathrm{mmol} \mathrm{L}^{-1} \mathrm{~h}^{-1}$ as typical values for the oxygen uptake rate in large-scale systems. The critical dissolved oxygen concentration for bacteria is about $1 \%$ to $5 \%$ of the saturated oxygen concentration in cultivation media for aeration with air.

\subsubsection{Oxygen transfer in aerated bioreactors}

Oxygen is only poorly soluble in water. The saturated dissolved oxygen concentration in water at $25^{\circ} \mathrm{C}$ aerated with air at ambient pressure is approximately $7 \mathrm{mg} \mathrm{L}^{-1}$ (Shuler and Kargi, 1992). The presence of dissolved salts and organics can alter the solubility. The influence of various medium components on the solubility has been investigated by Schumpe et al. (1982). 
Because of the low solubility of oxygen in culture media, the mechanism of oxygen transfer from the bulk of the gas phase into the bulk liquid suspension of single cells is controlled by the liquid phase mass transfer resistance (Reuss, 1993). This is also reflected by the commonly used film model which assumes a laminar film layer on either side of the interface between the gas bubble and the liquid phase (Figure 3-3). According to this model, the mass transport through these layers is diffusive with constant mass transfer coefficients $k_{g}$ and $k_{L}$ in the gas film or the liquid film, respectively. The model further assumes locally constant oxygen concentrations in the gas bubble and in the liquid bulk (ideally mixed conditions), and thermodynamically balanced conditions at the interfacial transfer area.

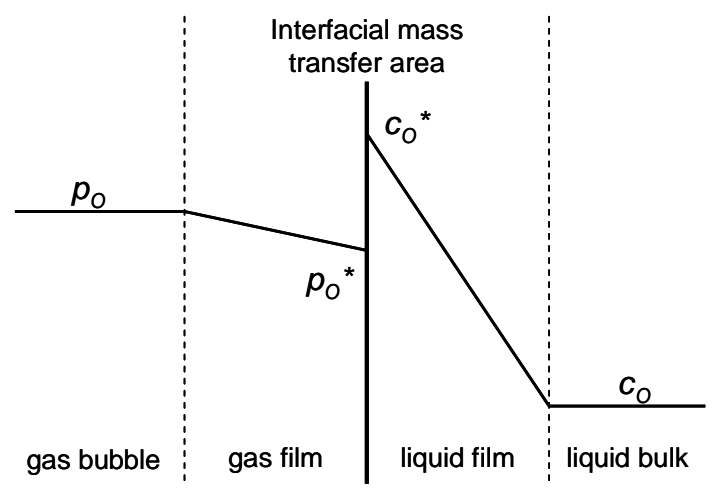

Figure 3-3: Film model for the oxygen transfer from gas bubbles into the liquid phase (refer to the text for a description).

The oxygen flux through the gas film depends on the gradient of the oxygen partial pressure $\left(p_{O}-p_{O}{ }^{*}\right)$ between the bulk of the bubble and the gas/liquid interface and on the mass transfer coefficient $k_{g}$, while the oxygen flux in the liquid film depends on the gradient of the oxygen concentration $\left(c_{O} *-c_{O}\right)$ between the interface and the liquid core and on the mass transfer coefficient in the liquid phase $k_{L}$. Since the mass transfer coefficient of oxygen in gas is significantly larger than that in the liquid phase, the mass transfer resistance in the gas film can be neglected. The oxygen mass flow from the gas into the liquid at balanced conditions can then be described as a function of the concentration gradient in the liquid and the transfer coefficient $k_{L}$. For the description of the oxygen transfer rate $O T R$ in a bioreactor, which expresses the total oxygen flux through the interfacial area of all gas bubbles with respect to the total reactor volume, the volumetric interfacial mass transfer area $a$ is introduced to yield Equation 3-26.

Equation 3-26: $\quad$ OTR $=k_{L} a \cdot\left(c_{O} *-c_{O}\right)$

As the oxygen concentration gradient $\left(c_{O}^{*}-c_{O}\right)$ in the liquid is the driving force for the oxygen transfer, the oxygen transfer rate can be improved by increasing 
the saturated oxygen concentration $c_{O} *$. According to Henry's Law (Equation 3-27), this can be achieved either by enriching the gas flow for aeration with oxygen or by elevating the total pressure of the system, because according to Dalton's Law the partial pressure $p_{O}$ depends on the molar fraction $\gamma_{O}$ of oxygen in the gas and on the total pressure $p$ (Equation 3-28). Hence both strategies result in an elevated partial oxygen pressure $p_{O} *$ at the interface between the gas and the liquid phase at saturation.

Equation 3-27: $\quad c_{O}^{*}=K_{H, O}(T) \cdot p_{O} *$

with the Henry constant for oxygen $K_{H, O}$, which depends on the temperature $T$.

Equation 3-28: $\quad p_{O}=\gamma_{0} \cdot p$

Alternatively, the oxygen transfer rate can be enhanced by increasing the volumetric oxygen transfer coefficient $k_{L} a$. This can be achieved by intensified agitation for a better dispersion of gas bubbles in the liquid, which results in an increase of the specific interfacial mass transfer area. Furthermore, a higher power input reduces the film layer between gas and liquid phase and thus increases the mass transfer coefficient $k_{L}$.

\subsection{Scale-up and scale-down of aerobic bioprocesses}

This section introduces general considerations for the design of stirred tank reactors for aerobic cultivations and describes microbial responses to gradients of substrate concentrations in large-scale reactors or in their scaled down models.

\subsubsection{Approaches for scale-up of aerated bioreactors}

A bioreactor must provide the proper physical and chemical environment, for example temperature, $\mathrm{pH}$, and substrate concentration, for the cells and must ensure fast transport of substrates and products between gas phase, bulk medium and cells with as low as possible effort in material, energy, and mechanics. In a stirred tank reactor, the most common vessel for biological production processes, the mechanical energy for mixing is supplied by a rotating impeller, which is also responsible for the gas dispersion. Gas is usually provided to the reactor via spargers which lead the gas flow close to the impellers where the local energy dissipation is highest. Generally stirred tank reactors are equipped with baffles located on the inside of the vessel walls designed to enhance turbulence and improve mixing. The upper limit for practicable volumes in stirred tank reactors is in the range of several $100 \mathrm{~m}^{3}$. 
Design goals for stirred tank reactors are good mixing properties resulting in low gradients of dissolved reactants and temperature, good heat exchange, and low costs of investment (Bellgardt, 2000). As most microbial production processes aim for high cell concentrations for high product titers, a sufficient oxygen transfer rate from the gas phase into the liquid phase is one of the most critical aspects for a successful aerobic cultivation. Consequently most of the common criteria for the scale-up of stirred bioreactors are more or less closely related to the oxygen supply to the organisms in the culture medium. These criteria include the volumetric oxygen transfer coefficient $k_{L} a$, the volumetric power input, the volumetric gas flow, the impeller tip speed or agitation speed, and the terminal mixing time. Much has been written about the principle of "similarity" for performing scale-up. This concept, however, can seldom be directly applied because the important similarity states (geometric, kinematic, and dynamic) are virtually impossible to maintain when going from laboratory to large scale operation (Reuss, 1993).

The liquid phase in stirred tank bioreactors is often assumed to be ideally mixed in order to simplify the modeling of mass and heat transfer phenomena. However, the mixing time in a reactor generally increases with scale and thus large fermentors often have regions that are not well-mixed. Although scale-up models and the use of characteristic time analysis are potentially attractive, a more immediate approach to the rational scaling of reactors is scale-down. The basic concept is to provide an experimental system at a smaller scale that duplicates exactly the same heterogeneity in environment that exists at larger scale. At a smaller scale, many parameters can be tested more quickly and inexpensively than at the production scale. Also, such a small-scale system can be used to evaluate proposed process changes for an existing operating process (Shuler and Kargi, 1992).

More than twenty years ago, Oosterhuis et al. (1985) simulated production-scale conditions with respect to oxygen gradients by employing a two-compartment system, of which one compartment could be gassed with pure oxygen. The investigations in this system allowed valuable conclusions about oxygen uptake, growth and product formation rates of the studied organisms under different oxygenic conditions. The authors highlighted the necessity of scale-down experiments for large-scale process optimization. Enfors et al. (2001) published the summarized results of several collaborating research groups who studied various aspects of the heterogeneity observed in large scale reactors using high-cell density $E$. coli cultures. They used a scale-down reactor furnished with a high-glucose concentration zone, mimicking the conditions in the feed zone of the large bioreactor. A progressive change in cell physiological state with respect to cytoplasmic membrane integrity was reported. Onyeka et al. (2003) investigated the additional impact of the $\mathrm{pH}$ control via different strategies for the addition of aqueous ammonia into 
this system. The same two-compartment reactor was previously already described by George et al. (1993) for studies of the metabolism in Saccharomyces cerevisiae. Amanullah et al. (2001) used a similar scale-down approach to simulate and study spatial $\mathrm{pH}$ variations for cultivations of Bacillus subtilis in large-scale bioreactors. Papagianni et al. (2003) designed a tubular loop reactor in order to imitate the circulation loops observed inside stirred tank reactors.

In all of these studies, the use of scale-down models allowed the investigation of the effects of defined heterogeneities, expected or observed at large scale bioreactors, on the cellular metabolism or productivity of the used microorganisms.

\subsubsection{Effects of substrate fluctuations in microbial cultivations}

The dynamics of a biological process have time scales of significantly different magnitude. Quite fast phenomena with time constants below seconds are catabolic reactions in the cells and local mixing due to turbulent flow. The circulation time through the entire reactor ranges from seconds up to minutes, depending on the reactor size and mixing characteristics, while the growth process proceeds in the range of hours (Bellgardt, 2000).

Inadequate mixing in large-scale reactors can cause localized gradients in largescale reactors with respect to temperature, $\mathrm{pH}$ and the concentration of the added substrates, i.e. mainly the carbon source and oxygen for aerobic processes. A pocket of liquid caught behind a baffle could easily become oxygen-depleted. Possible consequences of these short anaerobic periods are discussed by Konz et al. (1998). The authors relate the importance of oxygen to both its primary use as a nutrient and its secondary effects on metabolism and physiology. They point out that the shift from exponential to linear growth due to oxygen depletion, regarded as partial anaerobiosis or "microaerobic growth", can have significant impact on the physiology of the culture.

On the other hand, the same authors mention the exposure to elevated oxygen partial pressure as a source of potential scale-up problems. Typically, shake flask experiments and bench-scale bioreactors are run with ambient air. With scale-up, however, cells can encounter higher oxygen pressures for several reasons. Firstly, to avoid oxygen transfer limitation, bioreactors may be sparged with oxygenenriched gas. Secondly, in large bioreactors, the sparged air pressure may be over an atmosphere higher than in the head space due to the liquid head. Finally, the head space pressure of bioreactors may be chosen above ambient pressure to facilitate maintenance of sterility. These factors may result in localized oxygen partial pressures that are several-fold higher than those experienced in shake flasks or bench-scale reactors where the initial process development was done. 
The generation of reactive oxygen species like superoxide, hydrogen peroxide or hydroxyl radical may harm the cells. Konz et al. (1998) review potential damages, including metabolic changes, protein oxidation, DNA oxidation, and plasmid replication. Oosterhuis et al. (1984) reported diminished growth and product formation rates for Gluconobacter oxydans after short residence times in a reactor compartment which was gassed with pure oxygen.

In addition to the inhomogeneous distribution of oxygen or other gases in large scale fermentors, the gradients in the concentration of the added feed during fedbatch processes have to be considered at a technical scale. Obviously, substrate concentrations are highest next to the feeding port. In other parts of the reactor there may be zones with only very low or zero substrate concentration. Microorganisms moving through the vessel may be exposed to substrate limitation or even starvation in the latter zones and peak concentrations well above the average substrate concentration in the area near to the feed inlet. Depending on the size of the reactor and its mixing characteristics, the organisms experience these shifts on a timescale of a few minutes (Enfors et al, 2001). In regions with a high substrate concentration, the aerob consumption of this substrate can lead to local oxygen depletion at high cell densities, which may act as a stress factor and may cause increased by-product formation (Bylund et al., 1998). Bylund et al. (1999) reported decreased cell mass yields and enhanced by-product formation for glucose fluctuations between 5 and $4500 \mathrm{mg} \mathrm{L}^{-1}$ in E. coli cultivations. They attributed these observations not directly to the shifting glucose concentration, but to the secondary effect of probably occurring oxygen limitations due to increased glucose uptake rates in areas with greater substrate concentration.

Several groups have employed different strategies for the discontinuous provision of substrate, often in order to study the impact of fluctuating substrate concentrations on the physiology of various microorganisms. The results are diverse and sometimes contrasting. While Lin and Neubauer (2000) observed reduced productivity and growth at meanwhile higher viability for Escherischia coli in experiments with on/off feeding cycles of two minutes or 30 seconds, Weuster-Botz et al. (2001) reported increased productivity for $E$. coli at intermittent feed rates of six to seven minutes compared to continuously fed cultures. Bhargava et al. (2003) reported improved volumetric production of glucoamylase by Aspergillus niger when the substrate maltodextrin was fed for 90 seconds every five minutes. This was because more feed was added as soon as more dissolved oxygen was available. The increased amount of substrate consumed in this experiment also generated higher biomass concentration, but with the same growth yield on substrate.

There is certainly not the same physiological response of different microorganisms to substrate fluctuations. But it is very likely that cellular stress responses are 
triggered in any organism, if these fluctuations get drastic enough to lead to alternating over-feeding and starvation situations which last long enough to activate the respective physiological response. Teich et al. (1999) reported that E. coli reacted to glucose starvation with both the stringent response and the general stress response, indicated by the corresponding response regulators. Schweder et al. (1999) observed elevated mRNA levels for oxygen limitation genes immediately within seconds after $E$. coli cells entered glucose-rich, oxygen-depleted zones in a reactor. Konz et al. (1998) pointed out that the stress regulons have evolved to counter detrimental effects, so that the potential damage or limitation and the triggered cellular response have to be regarded as a dynamic system. For example, if the aeration is shifted from air to oxygen-enriched gas during the expression phase of a product, this may first lead to reduction of the product quality. Later, when the stress regulons are induced, the quality may increase again.

\subsection{Parallel experiments for bioprocess development}

This section provides the reason why it is necessary to employ parallel experiments for bioprocess development and then introduces various screening devices at different scales which are currently used for academic as well as for industrial purposes. The final subsection describes the particular screening setup used during this project.

\subsubsection{Intention for the use of parallel experiments}

When a biocatalytic reaction with potential industrial relevance is found, several phases of development have to be passed through until the actual production process is established, which is illustrated in Figure 3-4.

The phase of research includes the search for new or improved biocatalysts. Biocatalysts can be whole cells as well as free or immobilized enzymes. Nowadays the tools of metabolic and enzyme engineering are applied for the optimization of biocatalysts in the production of a wide range of pharmaceutically important molecules (Chartrain et al., 2000). The use of directed evolution has rapidly emerged to be the method of choice for the development and selection of mutated enzymes with enhanced properties. Directed evolution mimics natural evolution in the search of "fitter individuals", in this case improved variants of genes for enzymes with the activity of interest, and is directed in the sense that better variants are selected according to the set criteria (Marrs et al., 1999). It will clearly play a role in fine-tuning engineered metabolic pathways in the future, and in adapting whole-cell biocatalysts to the environment that is optimal for the stability and purification of the product (Hibbert et al., 2005). 
The optimization of process strategies during the development can generally be conducted either by changing one factor at a time or by varying several factors at the same time and looking for interactions using statistic analysis (Thiry and Cingolani, 2002). The use of a stochastic search strategy, for example the genetic algorithm, is particularly valuable when a large number of variables and a multimodal relationship exist for the target function (Weuster-Botz, 1999).

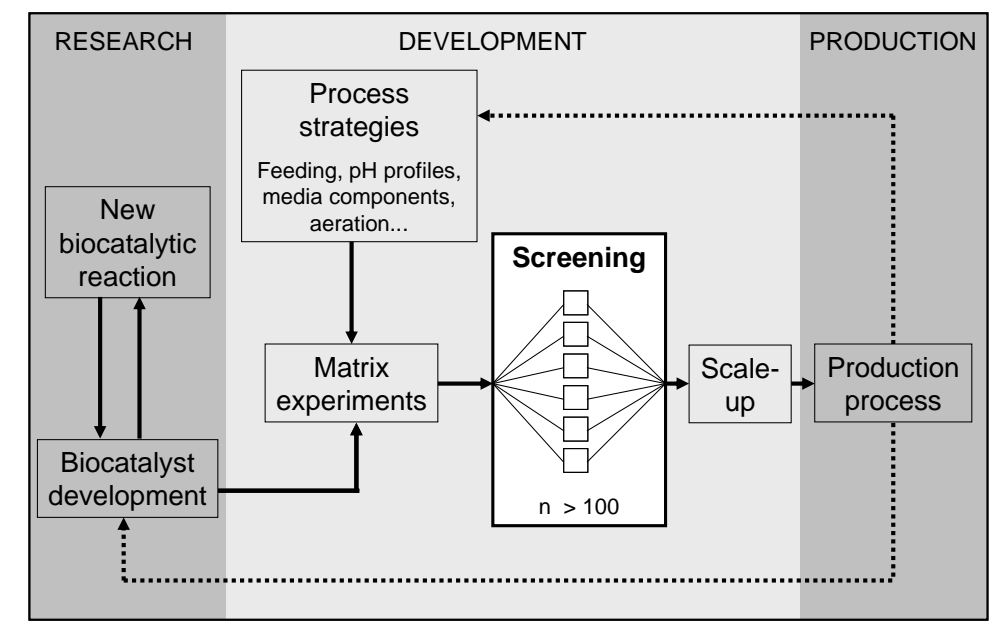

Figure 3-4: Schematic illustration of process development for biotechnological production processes. Biocatalysts can be whole cells as well as free or immobilized enzymes. The variety of different screening parameters leads to the design of matrix experiments which require a high number of parallel test runs (n). The dotted arrows indicate that also an established production process needs to be regularly revised and optimized regarding the used biocatalyst and the applied process strategy in order to remain competitive on the market.

The combination of numerous new or improved biocatalysts with various process strategies leads to matrix experiments with generally well over 100 single test runs (Freyer et al., 2004). In addition to the first screening cycles, which lead to the scale-up of the processes and to validated production protocols, also the established processes need to be revised regularly in order to remain competitive on the market.

The large number of screening experiments calls for fermentation devices suitable for highly parallel, preferably automated cultivations in order to save time and manpower. The key challenge in the evolution of automation is the ability to grow cultures on a much smaller scale, yet in a manner reflective of large-scale fermentors, which is where the new strain's productivity or other attributes must ultimately have their impact (Parekh et al., 2000). The required devices should thus allow modelling the setup relevant for production. Bellgardt (2000) defines a model as "an image of a real system that shows analogous behavior in the important properties, and that allows within a limited region a prediction of the behavior of the original system". The model can offer the advantages of being simpler, smaller, cheaper or faster than the original system. 
For the choice of the most suitable model device for a screening system, the stage during the course of the process development should be considered. Early on, when an enormous number of experiments need to be performed, the highest degree of parallelization is favorable, even if this usually implies a reduced similarity of the experiments with the envisioned production process. The further the development proceeds toward the final production protocol, the more important is the equivalence of the process conditions during the respective screening experiments to the actual production scale.

The following subsection provides a compilation of some of the established and some of the more recently developed systems intended for screening purposes. After the initial acknowledgement of the shake flask as the classic test vessel, the assortment is arranged by increasing reactor volume.

\subsubsection{Classic and novel parallel screening systems}

To date the most widely applied reaction vessel for parallel experiments is the incubated shaking flask, which is easy to handle, but usually offers very limited possibilities for substrate addition or process monitoring and control (Büchs, 2001). In recent years this system could be improved by instrumentation of the flasks and by a better characterization and understanding of the occurring mass transfer phenomena (Wittman et al., 2003; Anderlei et al., 2004).

In addition, various systems for screening experiments at different scales have been developed. A screening system with a high degree of monitoring and control capability is provided by a technology using microfluidic cell culture devices with six bioreactors per array (20-450 $\mu \mathrm{L}$ per reactor), of which several can be integrated in a robotic workstation with stations for fluidic control, sensing and incubation (SimCell ${ }^{\mathrm{TM}}$ technology by BioProcessors Corporation, Woburn, Massachusetts, USA; US Patent \#20050037485). At even smaller scale ( $5 \mu \mathrm{L}$ per unit), Zanzotto et al. (2004) reported almost equal growth of $E$. coli in a membraneaerated micro-bioreactor compared to cultivations in a $500 \mathrm{~mL}$ bench-top reactor operated at similarly low oxygen transfer rates ( $\mathrm{k}_{\mathrm{L}} \mathrm{a}$ values between $0.017 \mathrm{~s}^{-1}$ and $0.069 \mathrm{~s}^{-1}$ ). They monitored optical density, $\mathrm{pH}$ and dissolved oxygen.

A simpler approach is the use of shaken microtiter plates with 24, 48 or 96 wells, which also allow a high degree of parallelization while using easily available equipment and are thus very useful for screening steps early on during the course of development (Duetz et al, 2000; Bahia et al., 2005). However, parallel monitoring of process parameters as well as a parallel $\mathrm{pH}$ control or controlled substrate addition is still difficult in microtiter plates or not possible at all, which together with the substantial evaporation from the shaken plates often limits this system to the 
early applications which cannot simulate a production process. Several research groups worked on the refinement of microtiter plates regarding monitoring of process parameters and $\mathrm{pH}$ control (Elmahdi et al., 2003; Girard et al., 2001). Others developed methods for the measurement of the dissolved oxygen in microtiter plates (John et al., 2003) and characterized the oxygen transfer in shaken microtiter plates (Duetz and Witholt, 2004). An alternative approach for the gas input into 48-well plates was recently presented by Doig et al. (2005), who employed aeration through polyethylene frits at the base of the microtiter plate, yielding 48 miniaturized parallel bubble column bioreactors with a reaction volume of $2 \mathrm{~mL}$ each, and by Maharbiz et al. (2004), who established an electrochemical gas generation system for eightfold units with $250 \mu \mathrm{L}$ per micro-well along with a monitoring capacity for optical density and $\mathrm{pH}$, which could be controlled via the addition of carbon dioxide.

Kostov et al. (2001) described the design of a cuvette-based bioreactor $(2 \mathrm{~mL})$ and the monitoring of $\mathrm{pH}$, dissolved oxygen and optical density in this system. Danielson et al. (2004) used the same volume in shaken $15 \mathrm{~mL}$ culture tubes, which were modified with a baffle for improved oxygen transfer rates. Lamping et al. (2003) miniaturized a conventional baffled stirrer tank reactor to a working volume of $6 \mathrm{~mL}$, employing an impeller with three 6-bladed turbines for mixing and fiber optic probes for online measurements. The realization of automated operation of several of these reactors in parallel has not been published yet. Puskeiler et al. (2004 and 2005a) presented results of fully automated batch and fedbatch cultivations with $E$. coli in seven parallel milliliter stirred tank reactors in a setup for up to 48 parallel vessels. This system is described in more detail in the following subsection.

At a larger scale ( $200 \mathrm{~mL}$ working volume) up to 16 parallel bubble columns can be operated fully automated regarding continuous feeding and $\mathrm{pH}$ control (Altenbach-Rehm et al., 1999; Dilsen et al., 2001; Weuster-Botz et al., 2001b). This setup has been further improved by introducing stirrers to the system (WeusterBotz et al., 2002). DASGIP (Jülich, Germany) offers instrumented Erlenmeyer flasks or stirred flasks with magnetic stirrers for parallel operation, including the control of temperature, gas addition, and dosing of substrate or solutions for $\mathrm{pH}$ compensation. BioSpectra (Schlieren, Switzerland) announced the fully automated operation of 32 parallel bioreactors with $400 \mathrm{~mL}$ working volume. Already available are $6 \times 2 \mathrm{~L}$ stirred glass vessels from Biospectra, which can be fully automated and operated in parallel with even more monitoring, including for example the detection of exhaust gas concentrations. Similar systems are also available from other providers, like up to four parallel bench-top stirred tank reactors from Sartorius BBI Systems (Melsungen, Germany). 


\subsubsection{Automated setup with 48 stirred tank reactors at a milliliter scale}

One of the most popular methods for scaling-up is based on maintaining the volumetric oxygen transfer coefficient $k_{L} a$ constant (Reuss, 1993). It seems thus reasonable to use this criterion also as a measure of similarity for miniaturized process setups. Hence many of the small scale systems aim to attain $k_{L} a$ values similar to those obtained in technical scale stirred tank reactors, as they are intended to mimic the conditions during standard production procedures. Puskeiler et al. (2005a) compared the maximum $k_{L} a$ and biomass concentrations achieved in several of the setups described in the previous subsection. They reported the best performance for cultivations in parallel stirred bioreactors at a milliliter scale.

These miniature stirred tank reactors have been developed for the use with magnetically driven gas-inducing impellers (Puskeiler et al., International Patent Application PCT EP03/14752, 2003). They can be operated in a reaction block for up to 48 parallel vessels (arranged as six per row and eight per column), which is illustrated as a photograph in Figure 3-6 and as a cross-section through one column of eight parallel reactors in Figure 3-5.

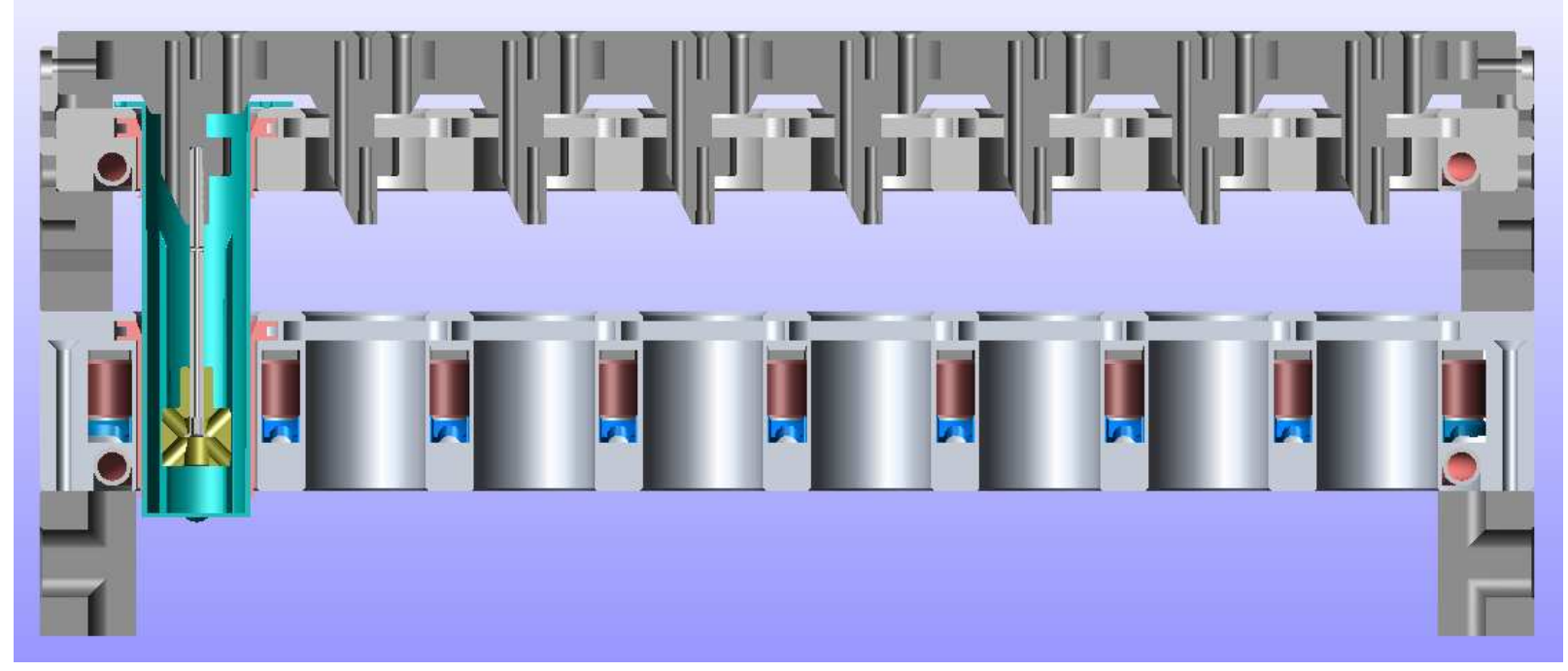

Figure 3-5: Cross-section through one column of the reaction block with one $\mathrm{mL}-$ reactor (green) and a magnetic turbine (yellow) on a central hollow axle. (Figure provided by $\mathrm{H}+\mathrm{P}$ Labortechnik AG, Oberschleissheim.)

The lower part of the reactors as well as the upper part (headspace) can be tempered separately to different desired temperatures. Aeration with sterile gas is through the cover lid into the headspace of the individual reactors, from where a fraction of the gas is sucked through two opposing holes in the upper part of the central hollow axles into the core of the magnetically driven turbines (gasinducing principle). There the gas phase meets the medium that is sucked in from the bottom, and the liquid phase, mixed with dispersed gas bubbles, is expelled 
through the diagonal channels of the impeller. This yields $k_{L} a$ values of up to $0.4 \mathrm{~s}^{-1}$, increasing with rising stirrer speed and decreasing liquid volume, assessed for liquid volumes between 7 and $14 \mathrm{~mL}$ (Puskeiler, 2004). Computational fluid dynamics simulation of single-phase flow in a volume of $11.2 \mathrm{~mL}$ yields a mean volumetric power input of $21.9 \mathrm{~W} \mathrm{~L}^{-1}$ at an impeller speed of $2800 \mathrm{rpm}$ (Puskeiler et al., 2005b).

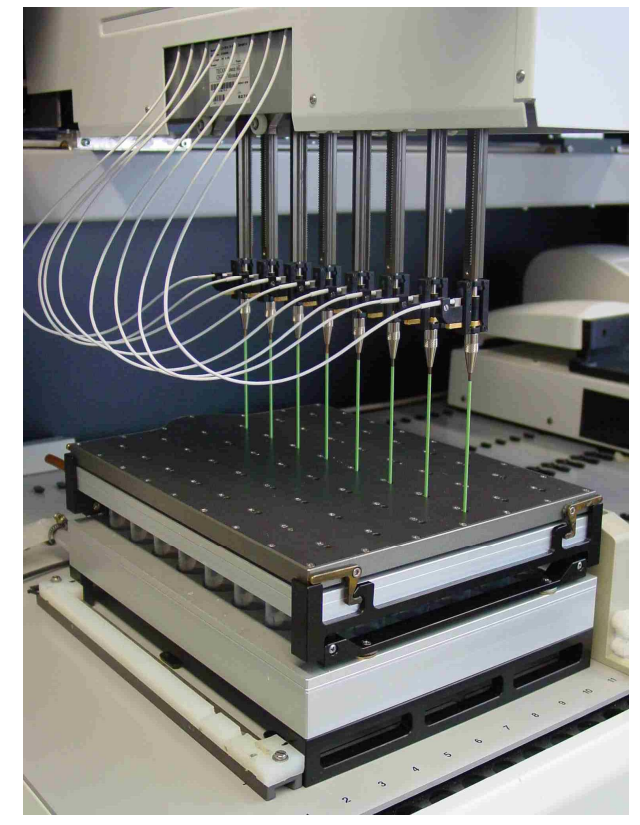

Figure 3-6: Photograph of the reaction block for up to 48 parallel milliliter reactors on the worktable of a laboratory robot with 8 individually controllable pipettes for liquid handling.

The setup is automated with a laboratory robot for intermittent liquid additions to realize feeding and $\mathrm{pH}$ adjustment and for parallel sampling from the reactors into microtiter plates for at-line analyses. The assessment of process samples takes place in a photometric plate reader. Furthermore the system comprises a plate washer for the recycling of the used microtiter plates. The control and communication of the individual hardware components can be integrated into the control software of the laboratory robot via software routines programmed in LabView (Puskeiler et al., 2005a).

This system allows the screening of a large number of parallel experiments under process conditions which resemble laboratory or industrial scale conditions with respect to the generated $k_{L} a$ and the degree of process monitoring and control. Cultivations can be carried out in batch or in fully automated intermittent fedbatch mode with automated parallel compensation of $\mathrm{pH}$ changes and evaporation loss (Puskeiler et al., 2005b). However, the discontinuous liquid addition implies modifications compared to the standard continuous fed-batch operation and causes concentration dynamics, which can possibly affect the physiology of the used microorganism and thereby the overall process performance. 


\subsection{Industrial production of riboflavin (vitamin B2)}

Riboflavin is a yellow, light-sensitive, water-soluble solid which has a molecular weight of $376 \mathrm{~g} \mathrm{~mol}^{-1}$. Its chemical structure is pictured in Figure 3-7. Riboflavin has the physiological role of a vitamin (vitamin B2) and serves as a precursor for the synthesis of the coenzymes flavin mononucleotide (FMN) and flavin adenine dinucleotide (FAD), which are needed as electron acceptors for many oxidoreductases (Perkins and Pero, 1993).

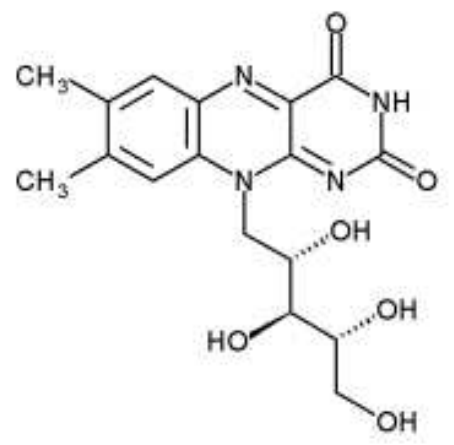

Figure 3-7: Chemical structure of riboflavin (vitamin B2).

Like the other vitamins of the B group, riboflavin supports energy production by aiding the metabolizing of fats, carbohydrates, and proteins. Whey, yeast, liver, kidney, egg yolk and green plants are major sources of riboflavin in human nutrition. Deficiency of vitamin B2 in men may cause inflammation of skin and mucosal membranes, disturbance of growth, and gastro-intestinal diseases (Brockhaus, 2000). Poultry and pigs react with growth retardation, poor utilization of feed, and diarrhoea to vitamin B2 deficiency (Stahmann et al., 2000).

Riboflavin is manufactured in bulk amounts for vitamin supplementation of human and animal nutrients. Since an excess riboflavin supply is harmless, because of efficient excretion via the urine, an important role for riboflavin is that of a colorant (E-101), for example for soft drinks and yogurt. In contrast to the highly purified riboflavin needed for multivitamin juices, colorant or pharmaceutical applications, more than $80 \%$ of the industrial production is used in a less pure form as animal feed additives (Stahmann et al., 2000). Fermentation processes are progressively replacing chemical manufacturing processes because of a significant cost reduction, and because they meet the "green" demand for replacing a mineral-oil-based process with one based on renewable resources (Stahmann et al., 2000; Van Loon et al., 1996). This development was initially prompted by the natural occurrence of bacteria, yeasts, and fungi, which produce riboflavin in levels exceeding their apparent metabolic requirements (Bacher et al., 2000). The key to the current competitiveness has been decades of strain development. 
When biotechnologically produced riboflavin entered the market in 1990, it contributed to about $5 \%$ of the global vitamin B2 market. In 2002 approximately $75 \%$ of the 4000 tons vitamin B2 market was produced via the cultivation of microorganisms (Karos et al., 2004). Three commercially relevant biotechnological processes are established using recombinant strains of either the hemiascomycete Ashbya gossypii (BASF, Ludwigshafen, Germany), the yeast Candida famata (Archer Daniels Midland, Decatur, IL, USA) or the bacterium Bacillus subtilis (DSM Nutritional Products, formerly Roche Vitamins, Basel, Switzerland) (Stahmann et al., 2000). Also strains of Corynebacterium ammoniagenes have been improved by means of metabolic engineering to produce more than $15 \mathrm{~g} \mathrm{~L}^{-1}$ riboflavin over 72 hours during repeated fed cultivations at laboratory scale ( 2 L) (Koizumi et al., 2000), which is comparable to the productivity reported for Bacillus subtilis (Perkins et al., 1999).

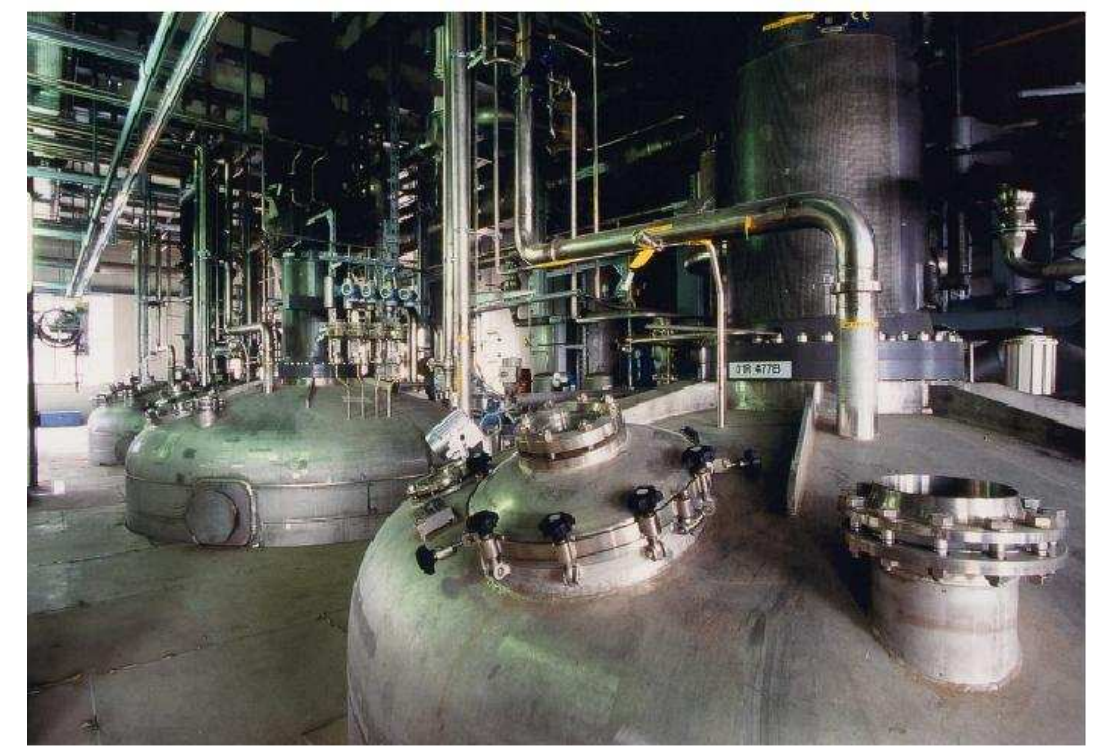

Figure 3-8: Production vessels for the biotechnological production of vitamin B2 (riboflavin) with recombinant Bacillus subtilis at DSM Nutritional Products, Switzerland.

During the process with Bacillus subtilis, the bacteria are grown in glucose-limited fed-batch cultivations at a $35 \mathrm{~m}^{3}$ scale (Bretzel et al., 1999) or larger. Figure 3-8 provides a picture of riboflavin production vessels at DSM Nutritional Products. Only minor amounts of the product remain inside the biomass, whereas most of the generated riboflavin accumulates in the culture medium, where it forms needle-shaped crystals due to its low solubility in neutral aqueous solutions (Van Loon et al., 1996) as represented in Figure 3-9 A+B+C.

After pasteurization of the fermentation broth, the crystals can easily be separated from the biomass using differential centrifugation. Subsequent washing in acid and re-crystallization provides a product with more than $99 \%$ riboflavin con- 
tent ("food/pharma-grade riboflavin"; Bretzel et al., 1999). The final formulation step leads to a powder product of vitamin B2 that dissolves in aqueous solution more easily than the needle-shaped crystals (Figure 3-9 D). The production of riboflavin with Bacillus subtilis meets at least the same product specifications and exceeds the quality criteria that are valid for the chemically synthesized products (Bretzel et al., 1999). More than 3000 tons per year of vitamin B2 are fabricated with this process.
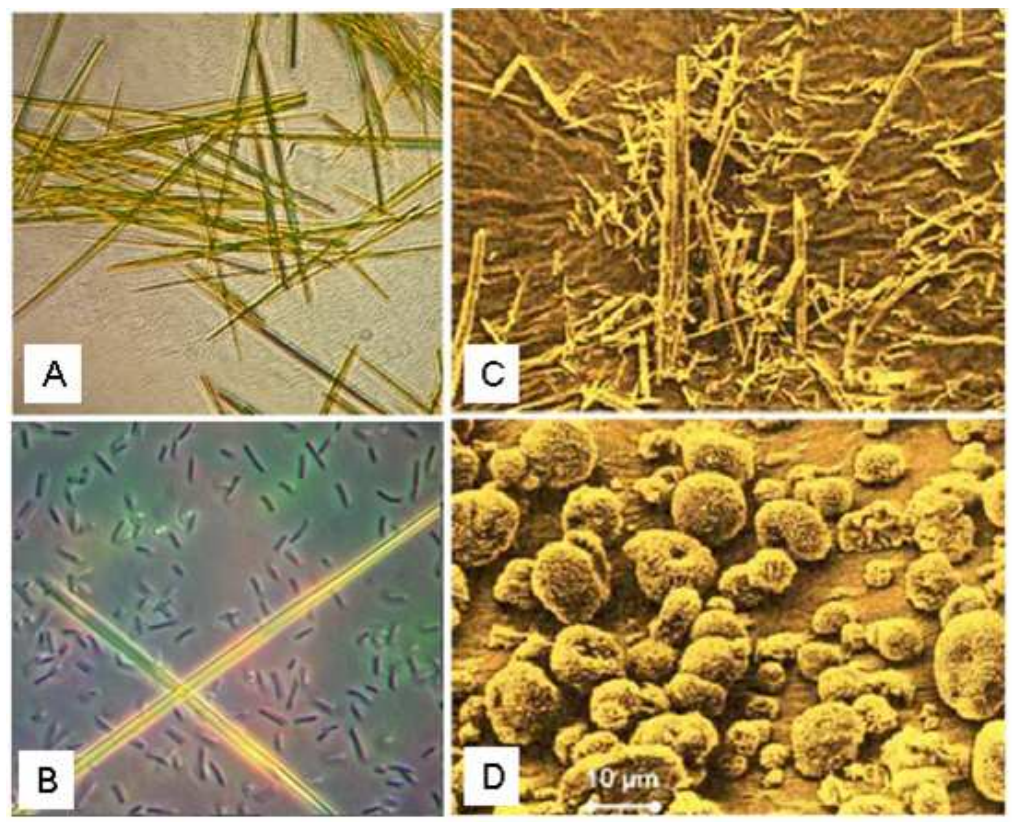

Figure 3-9: A and B: Microscopic photographs ( $40 \mathrm{x}$ and $100 \mathrm{x}$ magnification, respectively) of yellow needle-shaped riboflavin crystals generated during production processes with recombinant Bacillus subtilis (bacterial cells visible in B). C and D: Riboflavin crystals before and after the final formulation, respectively.

\subsection{Bacillus subtilis}

This section describes the Bacillus subtilis bacterium, in particular its metabolism. A major focus is on the biosynthesis of riboflavin and on the strategies for obtaining strains of this bacterium with up-regulated riboflavin productivity for the production of industrially relevant amounts of vitamin B2.

Bacillus subtilis is a gram positive, aerobically growing, rod-shaped bacterium, as illustrated in Figure 3-9 B. The wild-type Bacillus is motile and able to form endospores for the survival under hostile conditions (Priest, 1993). Species of the genus Bacillus play a dominant role in industrial bioprocesses (Schallmey et al., 2004). They produce a variety of different products, such as the fermented soybean product natto (Priest, 1993), several enzymes (Ferrari et al., 1993; Pero and Sloma, 1993), insecticides, purine nucleotides, vitamin B2 (riboflavin) (Perkins et al., 1999; Van Loon et al., 1996), and the flavor agent ribose (De Wulf et al., 
1997). The advantage of using Bacillus species for industrial purposes is that many of them are generally regarded as safe (GRAS) by the US Food and Drug Administration and that they grow rather fast which allows short production cycles (Schallmey et al., 2004). In addition the physiology and the genetics of Bacillus subtilis are well described, which allows directed metabolic engineering of the producer strains (Dauner and Sauer, 2001; Harwood and Wipat, 1996; Sauer et al. 1998; Zamboni et al., 2003).

\subsubsection{Central carbon metabolism in Bacillus subtilis}

The central pathways of carbon dissimilation via the glycolytic pathway and the tricarbonic acid (TCA) cycle, indicated with grey areas in Figure 3-10, are conserved in virtually all microorganisms.

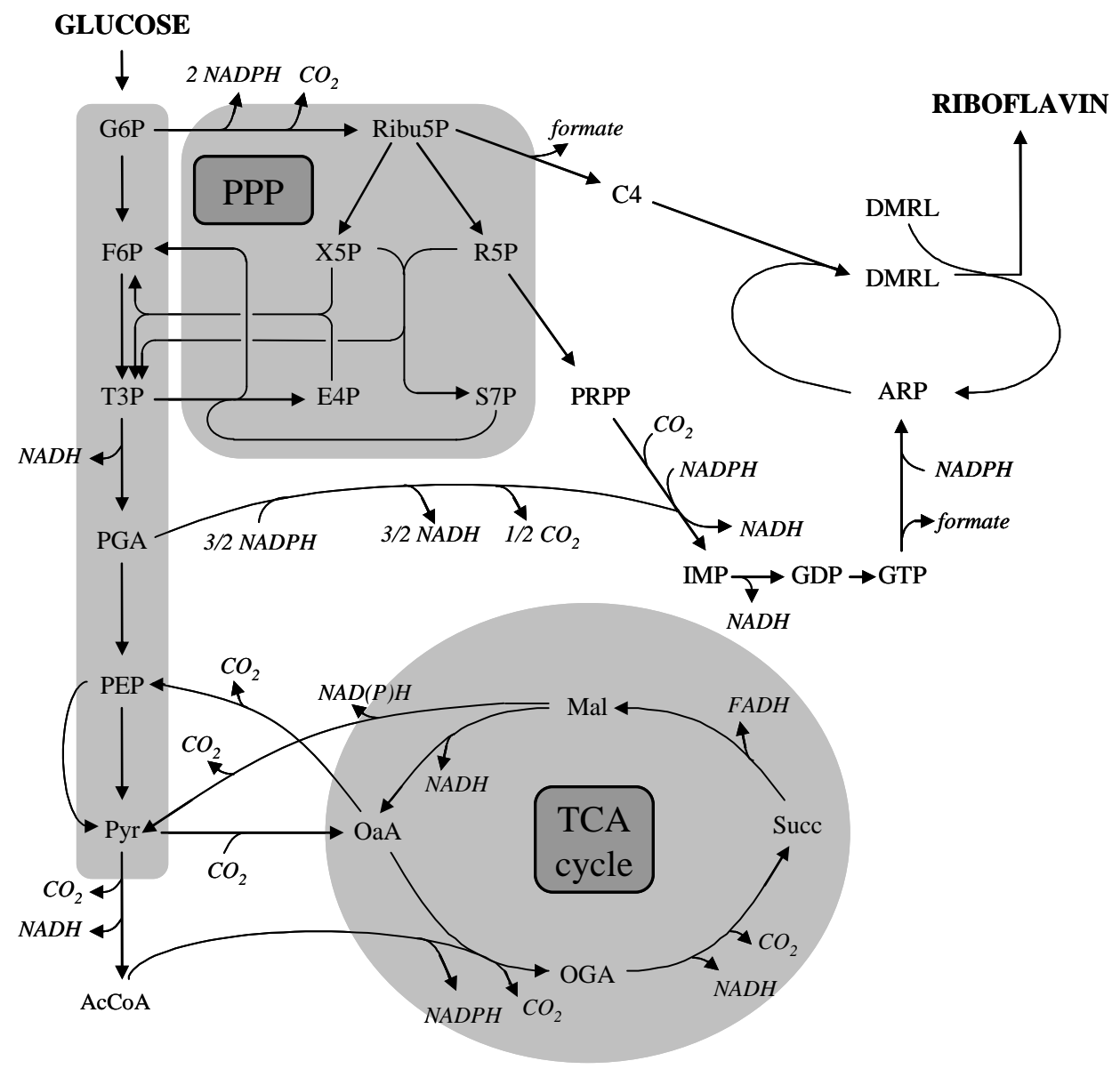

Figure 3-10: The biochemical reaction network in Bacillus subtilis (simplified from Sauer et al., 1996). Glycolytic reactions straight down to the left, pentose phosphate pathway (PPP), and the tricarbonic acid (TCA) cycle are shaded in grey. Abbreviations: ARP, 5-amino-6(ribitylamino)-2,4-(1H,3H)-pyrimidinedione; AcCoA, acetyl coenzyme A; C4, 3,4-dihydroxy-2-butanone; DMRL, 6,7-dimethyl-8-ribityllumazine; E4P, erythrose-4-phosphate; F6P, fructose-6-phosphate; G6P, glucose-6-phosphate; OaA, oxaloacetate; OGA, oxoglutarate; PEP, phosphoenolpyruvate; PGA, 3-phosphoglyceric acid; PRPP, phosphoribosyl diphosphate; Pyr, pyruvate; R5P, ribose-5-phosphate; Ribu5P, ribulose-5-phosphate; S7P, seduheptulose-7-phosphate; T3P, triose-3-phosphate; X5P, xylulose-5-phosphate. 
Not all of the substrate molecules that enter the glycolytic pathway are fully catabolized to carbon dioxide $\left(\mathrm{CO}_{2}\right)$ and water. Depending on the availability of nutrients, a significant fraction of the pyruvate or acetyl coenzyme $A$, generated by catabolism, may be converted to by-products that are excreted into the medium. The specific products are characteristic of the species, growth conditions, and growth phase (Sonenshein, 1993). For B. subtilis in chemostat cultures, these byproducts have been reported to be citrate, acetate, acetoin, butandiol, diacetyl or diacyl compounds (Amanullah et al., 2001; Dauner and Sauer, 2001; Dauner et al., 2001b).

Sauer et al. (1997) emphasized the importance of maintenance requirements of $B$. subtilis under conditions of slow growth. They reported maintenance coefficients of $0.08 \mathrm{~g} \mathrm{~g}^{-1} \mathrm{~h}^{-1}$ or $0.12 \mathrm{~g} \mathrm{~g}^{-1} \mathrm{~h}^{-1}$ for wild-type or recombinant Bacillus subtilis in chemostat cultures, respectively (Sauer et al., 1996). More recently also values of $0.21 \pm 0.04 \mathrm{~g} \mathrm{~g}^{-1} \mathrm{~h}^{-1}$ have been published by Dauner and Sauer (2001), using a model for the metabolic flux analysis which differed from the previous, for example in assuming different reaction reversibilities (Dauner et al., 2001a).

\subsubsection{Riboflavin biosynthesis in Bacillus subtilis}

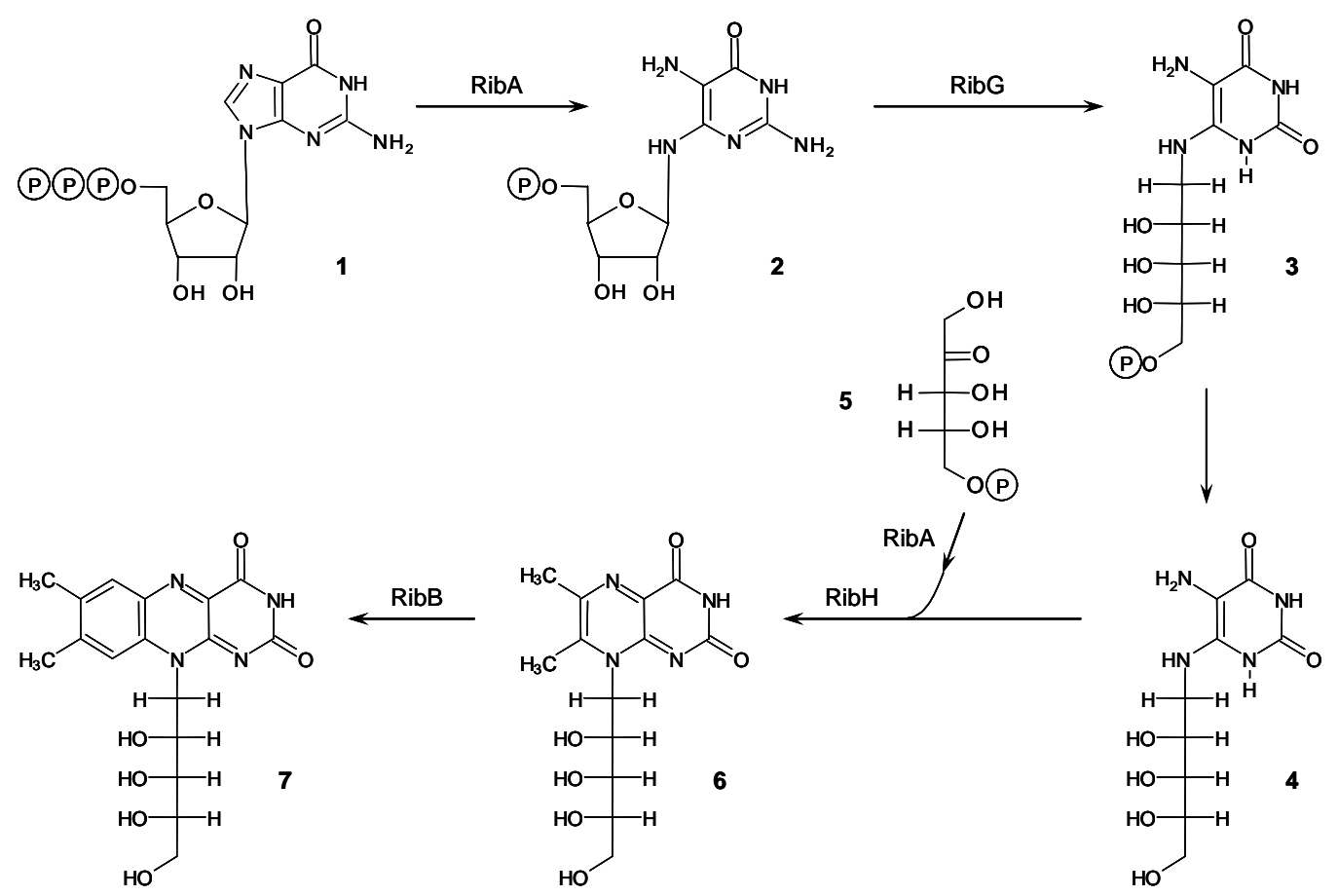

Figure 3-11: Riboflavin biosynthesis in bacteria (adapted from Bacher et al., 2000). Structures 1: guanosine triphosphate (GTP), 2: 2,5-diamino-6-ribosylamino-4(3H)-pyrimidinone 5'phosphate, 3: 5-amino-6-ribitylamino-2,4(1H,3H)-pyrimidinedione 5'-phosphate, 4: 5amino-6-ribitylamino-2,4(1H,3H)-pyrimidinedione, 5: ribulose 5-phosphate, 6: 6,7dimethyl-8-ribityllumazine (DMRL), 7: riboflavin. RibA, RibG, RibH, and RibB designate the gene products which catalyze the respective reactions. 
The biosynthesis of one molecule riboflavin requires one molecule of guanosine triphosphate (GTP) and two molecules of ribulose 5-phosphate as substrates. Figure 3-11 provides a simplified scheme of the biosynthetic pathway of riboflavin in bacteria.

The imidazole ring of GTP (structure 1) is hydrolytically opened, yielding a 2,5diaminopyrimidine (structure 2 ) which is converted to 5-amino-6-ribitylamino$2,4(1 \mathrm{H}, 3 \mathrm{H})$-pyrimidinedione (structure 4 ) by a sequence of deamination, side chain reduction and dephosphorylation. Condensation of 5-amino-6-ribitylamino2,4(1H,3H)-pyrimidinedione with 3,4-dihydroxy-2-butanone-4-phosphate, obtained from ribulose 5 -phosphate (structure 5) from the pentose phosphate pathway, affords 6,7-dimethyl-8-ribityllumazine (DMRL, structure 6). Dismutation of the lumazine derivative yields riboflavin (structure 7) and 5-amino-6-ribitylamino$2,4(1 \mathrm{H}, 3 \mathrm{H})$-pyrimidinedione (structure 4$)$, which is recycled in the biosynthetic pathway (Bacher et al., 2000).

In Bacillus subtilis, the genes for all the six enzymatic activities (RibA, RibG, RibH, RibB in Figure 3-11) that are involved in the riboflavin biosynthesis are clustered in the $4.3 \mathrm{~kb}$ riboflavin (rib) operon (Perkins et al., 1999). This is illustrated in Figure 3-12.
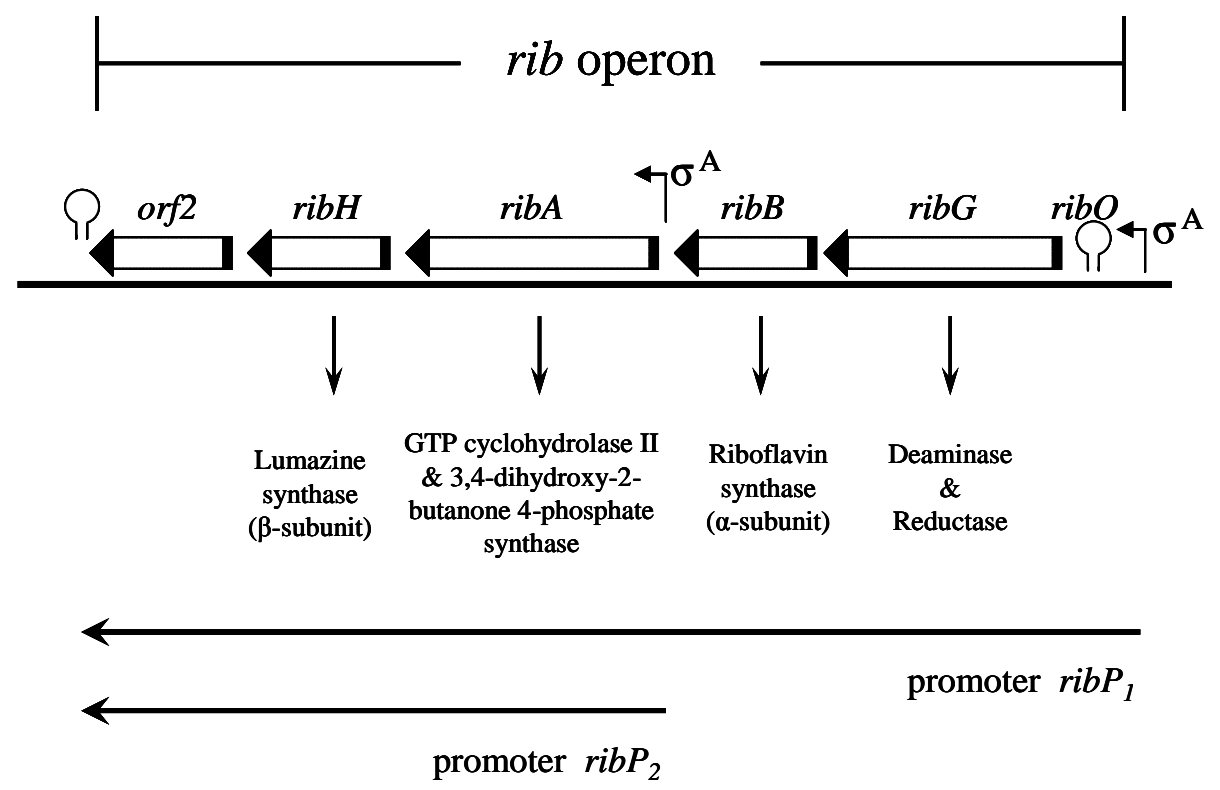

(A)

Figure 3-12: Simplified structure of the rib operon of Bacillus subtilis (adapted from Perkins and Pero, 1993). The rib operon hosts the four genes ribG, ribB, ribA and ribH (part A) encoding four proteins (part $\mathrm{B}$ ), which perform the biosynthesis of riboflavin. The open reading frame orf2 is of unknown function. The transcription of the genes is controlled by two promoters ribP $P_{1}$ and $r i b P_{2}$ (part C).

The order of genes differs from the order of enzymatic conversion steps. The ribA gene encodes GTP cyclohydrolase II activity, which catalyzes the first step in ribo- 
flavin biosynthesis (yielding structure 2 in Figure 3-11), and a second enzymatic function, which transforms ribulose-5-phosphate (structure 5 in Figure 3-11) to 3,4-dihydroxy-2-butanone 4-phosphate, an intermediate which is utilized later in the lumazine synthase reaction to yield DMRL. The ribG gene encodes for a bifunctional enzyme with deaminase and reductase activity for the second and third step of the synthesis, deamination of the pyrimidine ring and the subsequent reduction of the ribosyl side chain, yielding structure 3 in Figure 3-11. The $\mathrm{ribH}$ and the ribB gene encode for the enzyme activities that are responsible for the penultimate and for the final step of the pathway, the lumazine synthase and riboflavin synthase activity, respectively. The open reading frame orf2 following $\mathrm{ribH}$ at the $3^{\prime}$ end of the rib operon is of unknown function (Perkins et al., 1999).

The transcription of the genes in the rib operon is controlled by two promoter regions (part $C$ in Figure 3-12), which are recognized by the vegetative form $\left(\sigma^{A}\right)$ of the Bacillus subtilis RNA polymerase (Perkins et al., 1999).

An additional gene $r i b C$, which is located outside the rib cluster, plays an important role in the flavin metabolism of Bacillus subtilis (Mack et al., 1998). It encodes a flavin kinase converting riboflavin into the biologically active cofactors flavin mononucleotide (FMN) and flavin adenine dinucleotide (FAD) (Coquard et al., 1997). Mack et al. (1998) proposed that the flavin nucleotides, but not riboflavin, have an effector function for the regulation of riboflavin biosynthesis in Bacillus subtilis. The contrary is claimed by Nakajima (2004) who suggests that a rigid negative feedback inhibition is operative for the biosynthetic pathway of riboflavin in Bacillus subtilis, because in his experiments the supplementation of more than $250 \mathrm{ng} \mathrm{mL}{ }^{-1}$ riboflavin to the basal medium of a riboflavin-, adenine-deficient mutant completely restricted the formation of the precursor molecule DMRL.

Another riboflavin kinase is encoded by the ribR gene (Solovieva et al., 1999). This flavokinase is monofunctional and converts riboflavin into FMN but not to FAD. According to Solovieva et al. (2005), the expression of the ribR gene suppresses the riboflavin overproduction phenotype of Bacillus subtilis ribC mutant strains, which is a result of the strongly reduced flavokinase activity of the riboflavin kinase/FAD synthetase encoded by ribC.

Sauer et al. (1998) stated that maximum theoretical riboflavin yields may be achieved with Bacillus subtilis under process conditions that promote high carbon conversion efficiency, such as carbon-limited fed-batch cultivations, provided a thoroughly optimized product biosynthesis. The same authors also suggested the use of glycerol or sucrose as alternative carbon sources with reduced stoichiometric limitations. 


\subsubsection{Strain improvement of Bacillus subtilis}

Metabolic pathways in living organisms are regulated in a way to fit the cell's requirements in a given environment. However, when a certain compound is to be produced in industrially relevant amounts, the cell's requirements generally do not match the engineer's desires. Hence deregulated mutants have to be selected or the metabolic network has to be manipulated toward overproduction of the desired compound by using molecular biological techniques. According to this strategy Perkins et al. (1999) combined classical mutant selection with genetic engineering to yield Bacillus subtilis strains producing commercially relevant amounts of riboflavin. The following paragraphs summarize the major steps of their approach.

\section{Classical mutant selection}

Classical mutagenesis can be used to develop strains of various Bacillus species that are capable of producing significant quantities $\left(20-40 \mathrm{~g} \mathrm{~L}^{-1}\right)$ of inosine or guanosine (Debabov, 1982). As the riboflavin biosynthesis starts from guanosine triphosphate (GTP) (Bacher et al., 2000), the first part of the strategy for mutagenesis was to enhance the supply with purine precursors in Bacillus subtilis by selecting purine analog-resistant strains. Secondly, riboflavin analog-resistant mutants should be picked for a deregulated expression of riboflavin biosynthetic genes (Perkins et al., 1999).

Starting from Bacillus subtilis strain Marburg 168, mutants resistant to the purine analogs 8-azaguanine $\left(A z^{r}\right)$ and decoyinine $\left(D c^{r}\right)$ were achieved by subjecting the cells to ethyl methyl sulfonate (EMS) for mutations in the purine biosynthetic pathway to maximize the metabolic flux of carbon to GTP. The resulting strains had lost the ability to form endospores ( $5 P 00 A)$, which was transferred to the following strains. A further mutation (purA60) was introduced to block the flow from inosine monophosphate (IMP) to adenosine monophosphate (AMP) and related compounds. A strain, which carried all the three mutations, was exposed to the glutamine antagonist methionine sulfoxide (MS) for MS-resistant mutations yielding a limited degradation of IMP to inosine (Perkins et al., 1999).

Next, several strains containing multiple purine analog-resistant mutations were selected for resistance to the riboflavin analog roseoflavin ( $R_{0} F^{r}$ ) in order to deregulate the expression of these genes. In the resulting mutants both the 8azaguanine and the decoyinine resistance were necessary for enhanced riboflavin production (Perkins et al., 1999). The RoF ${ }^{r}$ mutation in one strain, designated RB50, could be mapped to the ribC locus (Coquard et al., 1997). RibC encodes the flavokinase activity for the conversion of riboflavin to FMN and FAD (Mack et al., 
1998). Missense mutations in ribC (such as RoF $^{r}$ in strain RB50) that alter the activity of the flavokinase also greatly enhance the expression of genes from both the $r i b P_{1}$ and $r i b P_{2}$ promoter (Perkins et al., 1999).

Genetic engineering of the Bacillus subtilis mutant strain RB50

The next step by Perkins et al. (1999) for improving the riboflavin productivity in Bacillus subtilis was the application of molecular biological techniques. This included firstly the construction of plasmids containing the rib operon and antibiotic resistance genes for selection. Additionally, the native promoters $r i b P_{1}$ and $r i b P_{2}$ were replaced with a strong constitutive promoter $P_{15}$, derived from the Bacillus bacteriophage SP01. These plasmids were introduced into the chromosome of $\mathrm{Ba}-$ cillus subtilis for the amplification of the gene dosage of riboflavin biosynthetic genes and for a further increase of their transcription (Perkins et al., 1999).

Table 3-1: Features of recombinant Bacillus subtilis RB50::[pRF69 $]_{n}::[p R F 93]_{m}$ which were achieved by a combination of classical mutant selection and genetic engineering for the production of commercially relevant amounts of riboflavin.

\begin{tabular}{|c|c|c|c|}
\hline Strain/Plasmid & Feature & Major Characteristics & Reference \\
\hline \multirow{5}{*}{$\begin{array}{l}\text { Bacillus subtilis } \\
\text { RB50 }\end{array}$} & $A z^{r}-11$ & resistance to purine analog 8-azaguanine & Perkins et al., 1999 \\
\hline & $D c^{r}-15$ & $\begin{array}{l}\text { resistance to purine analog decoyinine } \\
=>\text { GTP overproduction }\end{array}$ & Perkins et al., 1999 \\
\hline & purA60 & restriction of synthesis of AMP from IMP & Perkins et al., 1999 \\
\hline & $\mathrm{MS}^{r}$ & $\begin{array}{l}\text { resistance to methionine sulfoxide }=>\text { re- } \\
\text { striction of conversion of IMP to inosine }\end{array}$ & Perkins et al., 1999 \\
\hline & $\begin{array}{l}\text { RoF }^{r} \\
\text { (mutation } \\
\text { in ribC) }\end{array}$ & $\begin{array}{l}\text { resistance to riboflavin analog roseoflavin } \\
=>\text { reduced conversion of riboflavin to } \\
\text { FMN and FAD }\end{array}$ & $\begin{array}{l}\text { Coquard et al., } 1997 \\
\text { Mack et al., 1998; } \\
\text { Perkins et al., } 1999\end{array}$ \\
\hline \multirow{3}{*}{$\begin{array}{l}\text { pRF69 } \\
(n-\text { fold })\end{array}$} & rib operon & $\begin{array}{l}\text { hosts four genes encoding enzymes for } \\
\text { riboflavin production }\end{array}$ & \\
\hline & $P_{15}$ & $\begin{array}{l}\text { strong constitutive promoter replacing the } \\
\text { two native promoters }\end{array}$ & Perkins et al., 1999 \\
\hline & Cat & resistance to chloramphenicol & \\
\hline \multirow{3}{*}{$\begin{array}{l}\text { pRF93 } \\
\text { (m-fold) }\end{array}$} & rib operon & $\begin{array}{l}\text { hosts four genes encoding enzymes for } \\
\text { riboflavin production }\end{array}$ & \\
\hline & $P_{15}$ & $\begin{array}{l}\text { strong constitutive promoter replacing the } \\
\text { two native promoters }\end{array}$ & Perkins et al., 1999 \\
\hline & Tet & resistance to tetracycline & \\
\hline
\end{tabular}


Perkins et al. (1999) also reported that riboflavin is produced in higher levels when more than one copy of the rib operon is inserted into a Bacillus subtilis host strain, which implies that the general enzyme activity limits the riboflavin production rate. Clones harboring an amplified number of plasmids can be selected by increasing the concentration of the respective antibiotic in the growth medium. The riboflavin producing plasmids pRF69 and pRF93 contain either a chloramphenicol or tetracycline resistance marker, respectively. The integration of multiple copies of these plasmids into the chromosome of the mutant strain RB50 yielded Bacillus subtilis RB50::[pRF69 $]_{\mathrm{n}}::[\mathrm{pRF} 93]_{\mathrm{m}}$. This strain produced significantly more riboflavin during fed-batch fermentations than the original strain (more than $14 \mathrm{~g} \mathrm{~L}^{-1}$; Perkins et al., 1999), while its growth behavior remained unchanged compared to the original wild-type strain (Sauer et al., 1996). The genetic features of Bacillus subtilis RB50::[pRF69 $]_{\mathrm{n}}::[\mathrm{pRF93}]_{\mathrm{m}}$ are summarized in Table 3-1.

Further improvement of the riboflavin productivity by this strain could be achieved by reducing its maintenance metabolism, which was approximately $50 \%$ higher compared to the wild-type Bacillus subtilis in continuous chemostat cultures (Sauer et al, 1996), by the means of metabolic engineering. Zamboni et al. (2003) reported $40 \%$ reduced maintenance requirement accompanied by respectively increased riboflavin productivity in a cytochrome oxidase knockout mutant of Bacillus subtilis RB50::[pRF69]n. The authors made a redirection of the electron flow to more efficient proton pumping branches within the respiratory chain responsible for this result.

Another approach for enhanced riboflavin production in $B$. subtilis is presented by introducing an additional single copy of a constitutively expressed ribA gene, as RibA has been identified as the rate-limiting enzyme of riboflavin biosynthesis in an industrial Bacillus subtilis strain (Hümbelin et al., 1999). This led to an improvement of up to $25 \%$ of riboflavin yields on glucose. 


\section{Results and Discussion}

This chapter portrays the major results of the project, namely the establishment of the original riboflavin production process (B2 process) at laboratory scale as a reference (4.1), followed by the rationale for the necessary process modifications during an iterative scale-down (4.2), and the process performance under altered process conditions at laboratory scale (4.3). The developed process analyses for automated parallel at-line measurements in the milliliter setup are described (4.4) as well as the results for the automated at-line $\mathrm{pH}$ measurement and control (4.5). The next section (4.6) addresses physical, technical, and logistic aspects that were critical for the use of the automated milliliter setup, before the performance of the B2 process in this setup is presented along with some remaining concerns. Each of the subsections in this chapter concludes with a discussion of the provided results. The final section (4.7) provides a general guideline that can be applied for the scale-down of fed-batch processes to the milliliter setup.

\subsection{Original B2 process at laboratory scale (reference)}

The adaptation of the B2 process with Bacillus subtilis RB50::[pRF69] $]_{\mathrm{n}}$ (designated $B$. subtilis) from laboratory scale to the milliliter setup required operational modifications which were not supposed to change the original process performance, particularly with respect to the riboflavin production. Therefore, the B2 process was established in a $7.5 \mathrm{~L}$ standard stirred tank bioreactor according to the original fed-batch protocol from DSM Nutritional Products (DSM NP) in order to obtain a reference for the consecutive investigations.

Process parameters measured and controlled during two B2 cultivations, carried out essentially according to the original protocol, are presented as graphs in Figure 4-1 and Figure 4-2 with bold or thin lines for either process, respectively. Corresponding to this protocol, feed should be added at a constant rate of $41 \mathrm{~g} \mathrm{~h}^{-1}$ for an initial reactor volume of $3 \mathrm{~L}$ after the completion of the batch phase, which was indicated by a steep rise of dissolved oxygen. The actual feed addition, according to data of the feed balance, was $39.1 \mathrm{~g} \mathrm{~h}^{-1}$ or $39.6 \mathrm{~g} \mathrm{~h}^{-1}$ on average for either cultivation (thin or bold line in Figure 4-1 D, respectively). 

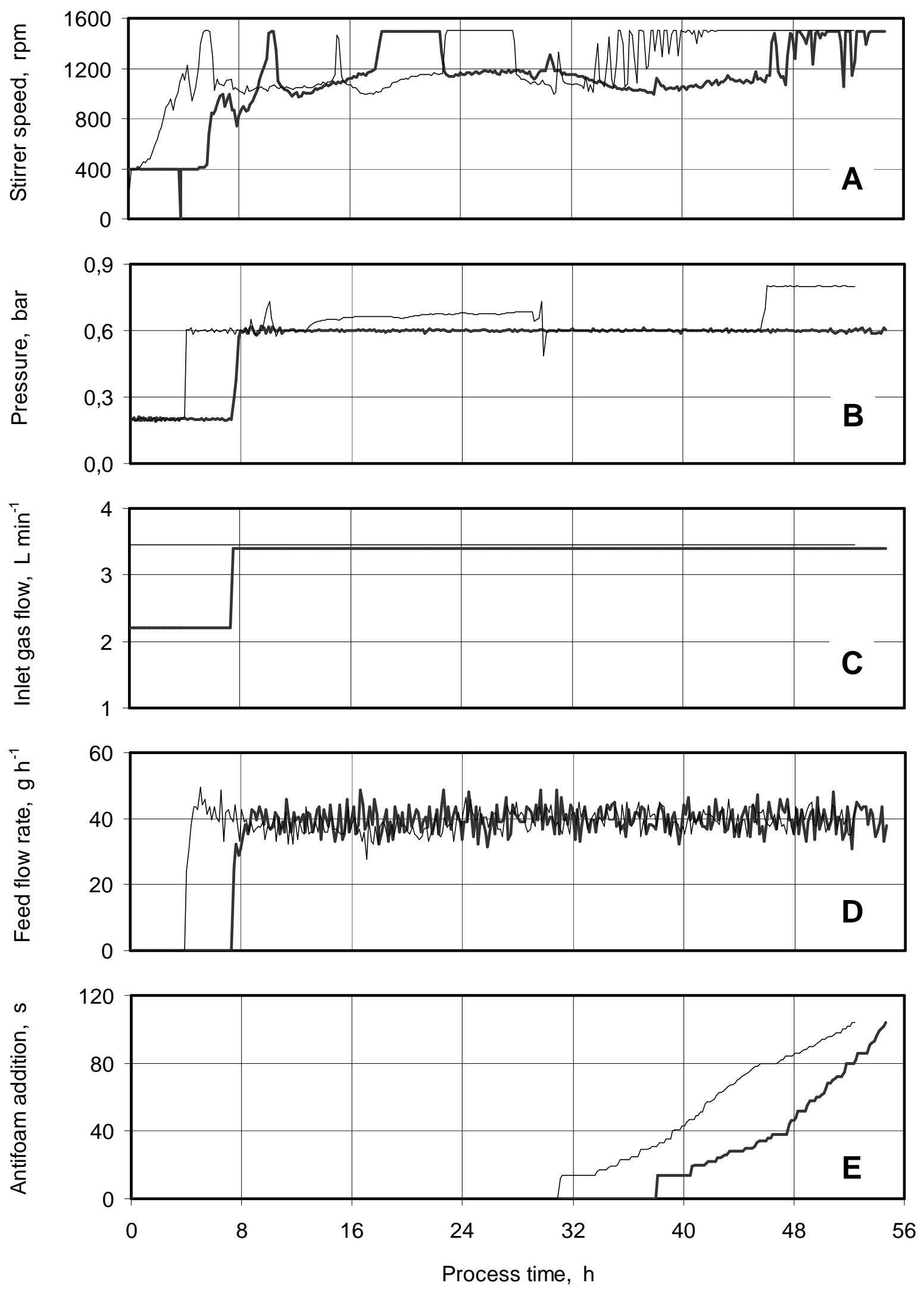

Figure 4-1: $\quad$ Stirrer speed (A), pressure (B), inlet gas flow (C), feed addition (D), and antifoam addition $(E)$ during two B2 processes at laboratory scale $(3 \mathrm{~L})$, carried out essentially according to the original protocol from DSM NP. The antifoam addition is expressed as the accumulated duration of the active times of the antifoam pump. Graphs for the feed flow rate are based on the feed consumption according to the feed balance. 
During the cultivation represented with bold lines, the start of the feeding phase was at $7.5 \mathrm{~h}$ process time which was approximately three hours delayed compared to the other process. This postponement is reflected in all of the graphs in Figure 4-1 as well as in those of the offline data (Figure 4-3).

The intended aeration was with a constant air flow rate of $3.4 \mathrm{~L} \mathrm{~min}^{-1}$. False adjustment of the air flow during the batch phase of the process with the delayed feed start resulted in 2.2 $\mathrm{L} \mathrm{min}^{-1}$ (Figure 4-1 C).
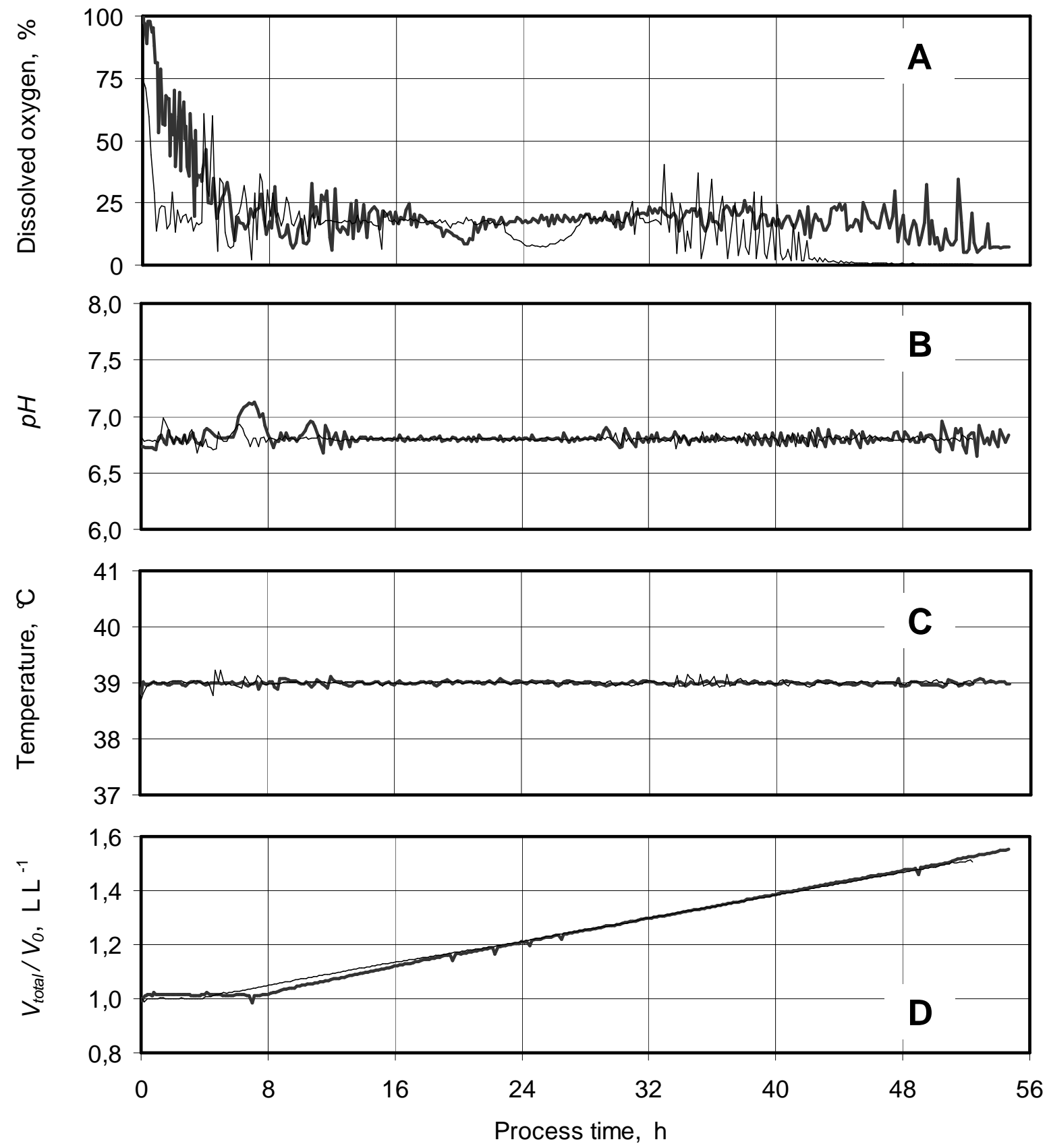

Figure 4-2: Online data during two $\mathrm{B} 2$ processes at laboratory scale, carried out according to the original B2 protocol. The displayed graphs for dissolved oxygen (A), $\mathrm{pH}(\mathrm{B})$, and temperature (C) are based on average values within intervals of ten minutes. The volumetric increase $(D)$ is expressed as the reactor volume $V_{\text {total, }}$, based on the online monitored reactor weight, divided by the initial volume $V_{0}$. 
The dissolved oxygen was allowed to drop down to $15 \%$ (Figure 4-2 A) and was to be maintained at this value by using a closed loop control for the stirrer speed. The stirrer speed during both processes reached its maximum of $1500 \mathrm{rpm}$ briefly approximately two hours after the feed start, then between $18 \mathrm{~h}$ and $22 \mathrm{~h}$ or $22 \mathrm{~h}$ and $27 \mathrm{~h}$, and towards the end of the processes (Figure 4-1 A) after the frequent addition of antifoam solution had started (Figure 4-1 E). The addition of antifoam solution was controlled by a conductivity sensor, which was used to detect foam buildup above a certain level of the liquid phase in the reactor.

Excess pressure of 200 mbar or 600 mbar should be applied during the batch or feeding phase, respectively, in order to meet the high oxygen need of the process for optimal riboflavin production. During the process represented with thin lines, the pressure control did not function properly during the first half of the feeding phase until more appropriate parameters for the respective PID control of the setup were employed at approximately $29.5 \mathrm{~h}$. At $46 \mathrm{~h}$ the pressure was raised to 800 mbar for increased oxygen transfer, because the dissolved oxygen could not be kept at $15 \%$ despite maximum stirrer speed (Figure 4-2 A).

The process software of the setup provided a PID control routine for the quasicontinuous adjustment of the online measured $\mathrm{pH}$ via the addition of $25 \% \mathrm{am}$ monium hydroxide. The $\mathrm{pH}$ during both cultivations could essentially be kept at its setpoint of 6.8 with minor temporary deviations $<0.2 \mathrm{pH}$ units (Figure 4-2 B). Only at the end of the batch phase during the process with the delayed feed start reached the $\mathrm{pH}$ shortly almost 7.2.

The temperature in the reactors was kept at $39^{\circ} \mathrm{C}$ during both experiments (Figure 4-2 C). The volumetric increase, describing the ratio of the total reactor volume $V_{\text {total }}$ to the initial reactor volume $V_{0}$ due to the addition of feed and base, was very similar during the cultivations and was approximately 1.5 at the end of both processes (Figure 4-2 D).

The graphs in Figure 4-3 depict the offline data of the processes. The process, represented with bold lines in Figure 4-1 and Figure 4-2, is presented with circles in these graphs, the other one with triangles. The batch glucose during the latter one was consumed during the first four to five hours (Figure 4-3 A). This period was also observed during several previous and consecutive B2 processes (data not shown). Significant glucose accumulation did not occur during the processes. The slower glucose consumption during the other process (circles) was accompanied by slower growth of $B$. subtilis (Figure 4-3 B). After 24 hours, however, the dry cell mass concentrations of both processes were in the same range (35$\left.40 \mathrm{~g} \mathrm{~L}^{-1}\right)$. At the end of the cultivations, approximately $46 \mathrm{~g} \mathrm{~L}^{-1}$ or $50 \mathrm{~g} \mathrm{~L}^{-1}$ dry cell mass were reached. 

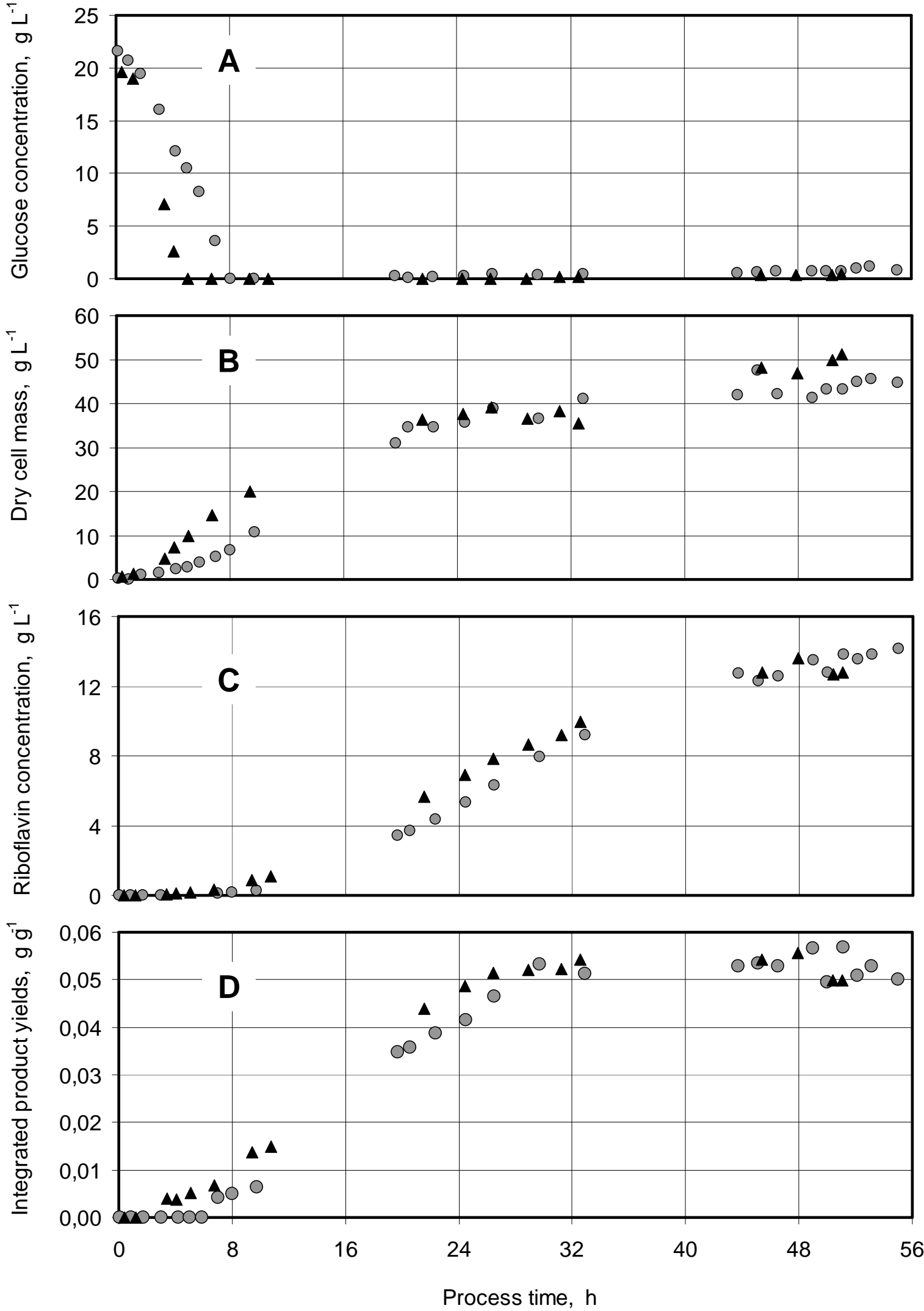

Figure 4-3: Glucose consumption (A), growth (B), riboflavin concentration (C) and product yields (D) generated by $B$. subtilis, based on the offline analysis during two B2 processes at laboratory scale (circles or triangles, respectively), carried out according to the original B2 protocol. Dry cell mass values are based on gravimetric determination. Riboflavin concentrations are based on the average of HPLC and absorbance measurements. 
During the cultivation represented with circles, also the riboflavin production was delayed by approximately three to four hours compared to the other process (Figure 4-3 C). However, the final product concentration was $13-14 \mathrm{~g} \mathrm{~L}^{-1}$ for both cultivations, based on the average values of HPLC and absorbance measurements of cell-free offline samples. The corresponding integrated product yields $Y_{P / S}$ were

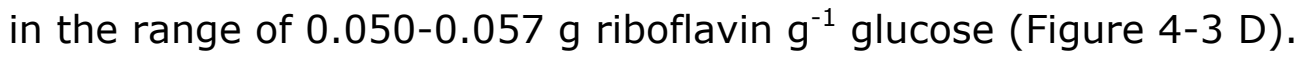

Specific growth and glucose uptake rates were maximal during the batch phases with approximately $0.8 \mathrm{~h}^{-1}$ and $2.2 \mathrm{~h}^{-1}$, respectively, and gradually decreased thereafter, while the specific riboflavin production rates quickly increased at the end of the batch phases to values between $0.012-0.013 \mathrm{~h}^{-1}$ and started to decrease after 24 to 28 hours, reaching minimal values at 48 hours (data not shown).

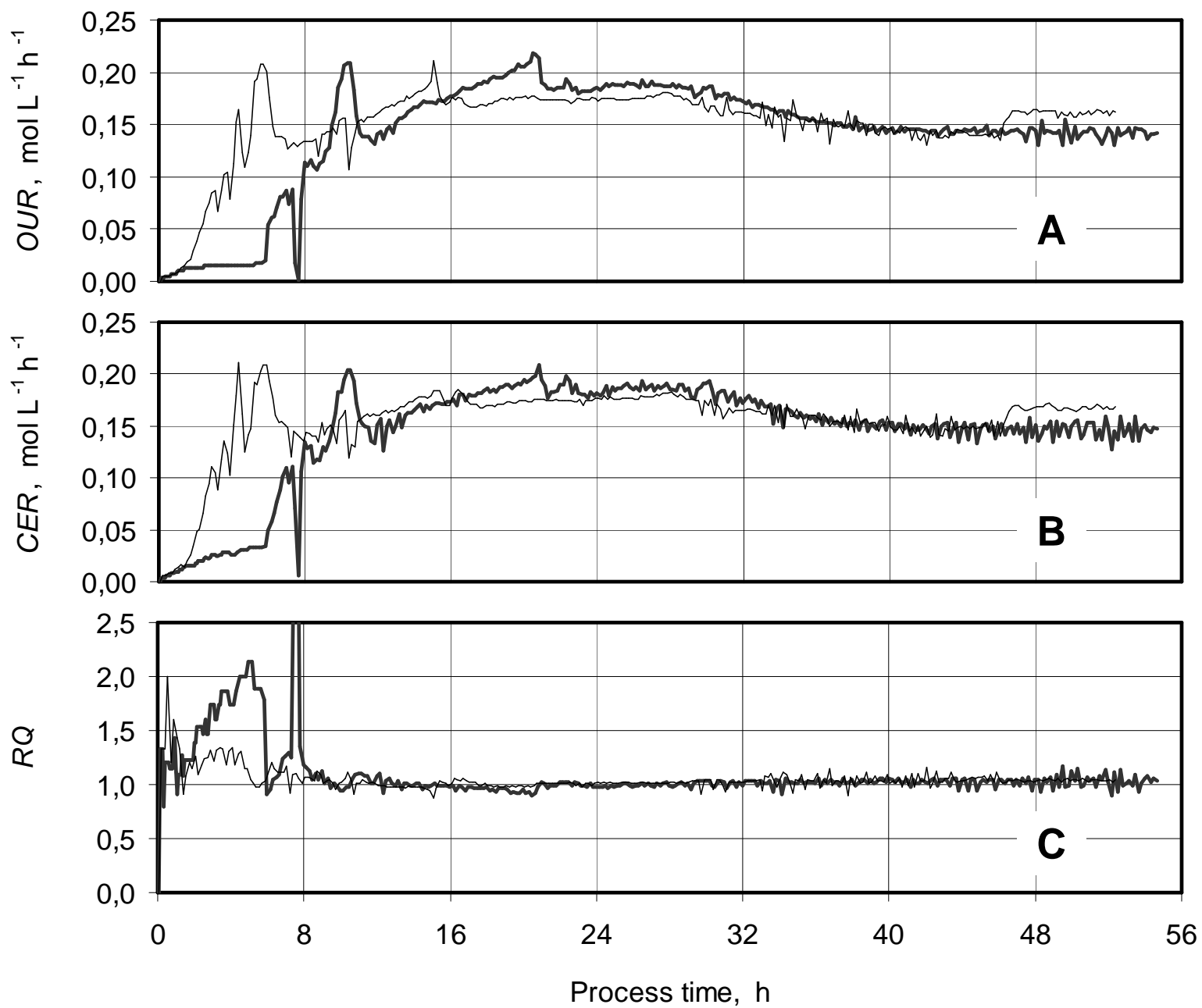

Figure 4-4: Oxygen uptake rates OUR $(\mathrm{A})$, carbon dioxide evolution rates $C E R(\mathrm{~B})$, and respiratory coefficients $R Q(\mathrm{C})$ for two $\mathrm{B} 2$ processes at laboratory scale, carried out according to the original B2 protocol. The displayed values are based on concentration balances in the gas flow, assuming the same values for the respective inlet and outlet gas flow rates. 
The analysis of the oxygen and carbon dioxide concentrations in the outgoing gas flow allowed balancing these components in the gas phase, so that the oxygen uptake rates OUR and carbon dioxide evolution rates CER for the cultivations could be estimated (Figure 4-4 A+B). They increased during the batch phase with oscillations due to the sequential consumption of glucose and other carbon sources and reached maxima of approximately $0,22 \mathrm{~mol} \mathrm{~L}^{-1} \mathrm{~h}^{-1}$ after $6 \mathrm{~h}$ and $15 \mathrm{~h}$ or $10 \mathrm{~h}$ and $20 \mathrm{~h}$, respectively. During major parts of the experiments the rates were in the range of $0,14-0,19 \mathrm{~mol} \mathrm{~L}^{-1} \mathrm{~h}^{-1}$. Apart from the observed delay for the process represented with bold lines, the profiles were very similar during both processes. The steep drop in the bold profiles at $7.5 \mathrm{~h}$ was caused by a short delay between the final consumption of the carbon sources and the initiation of the feed addition, which resulted in an erratic respiratory coefficient $R Q$ at this time (Figure 4-4 C). The respiratory coefficients rose to 2.1 during the batch phases of both processes and then quickly decreased to values around 1.0 until the end.

Carbon balances for both processes were calculated by dividing the outgoing amount of carbon $C_{\text {out }}$ by the ingoing amount of carbon $C_{i n}$, using on one side the carbon provided via glucose and yeast extract in the batch medium and in the feed solution, and on the other side the carbon generated as biomass, riboflavin, or carbon dioxide, or remaining as glucose in the reactor. The sum of all ingoing and outgoing carbon compounds should be equal. Therefore, the carbon balances should be close to $100 \%$, if all the generated carbon-containing compounds were detected completely.
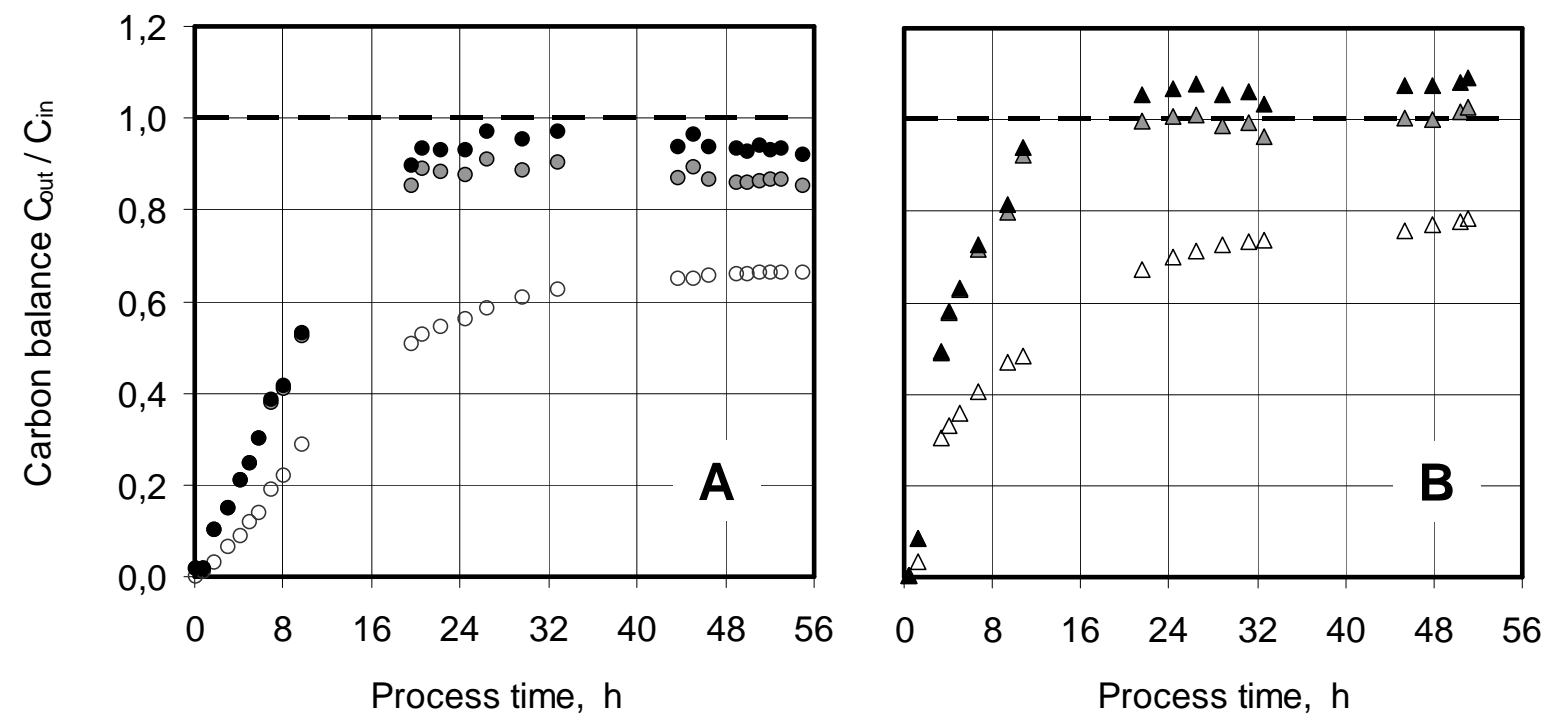

Figure 4-5: Partial carbon balances for two B2 processes at laboratory scale, carried out according to the original B2 protocol ( $\mathrm{A}$ or $\mathrm{B}$ respectively). The ingoing carbon $C_{\text {in }}$ originated from glucose and yeast extract. The values for the outgoing carbon $C_{\text {out }}$ derived from generated compounds carbon dioxide (open symbols) or carbon dioxide and biomass (grey symbols), or carbon dioxide, biomass and riboflavin (black symbols). The dashed lines indicate $100 \%$ analytical recovery of all the carbon that went into the processes. 
Figure 4-5 shows that the major part of the provided carbon during the B2 processes went into carbon dioxide, namely $65-75 \%$ at the end of the cultivations. 20-25\% of the carbon was found in biomass, and only 6-7\% was recovered as riboflavin. Further carbon-containing products, for example overflow metabolites, were not detected and could not be considered for the balances. Yeast extract remained in the medium until it was completely consumed. By definition, its residual concentration would be part of the outgoing carbon. But as it was not measured, all of it was always considered for the ingoing carbon, accounting for balances starting apparently close to zero at the beginning of both cultivations.

Figure 4-5 B revealed a total carbon balance clearly larger than $100 \%$ during the respective process (black triangles). Since this can not be true, the analytical results during this cultivation were questionable. Most likely the carbon dioxide concentrations were incorrect. The balance values of 92-97\% during the other process (black circles) were acceptable within the limits of accuracy and regarding the fact that analyses for by-products were not conducted.

\section{Discussion}

Both of the presented processes did not perform fully according to the original B2 protocol. The inconsistent carbon balance of the process represented with thin black lines and triangles undermined the reliability of the analytical data generated during this cultivation. In addition, the course of the applied excess pressure during this experiment differed from the original protocol. It should therefore not serve as a reference for further investigations.

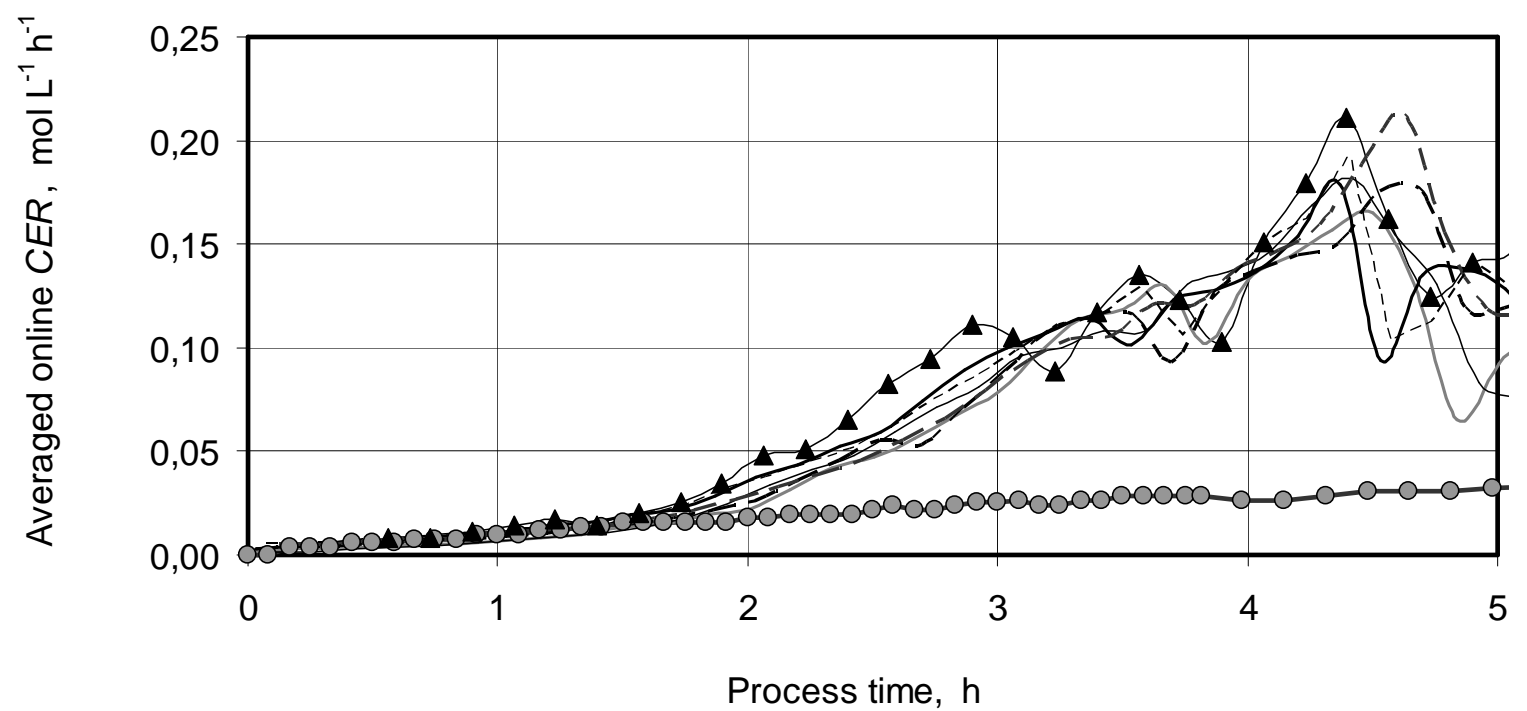

Figure 4-6: Carbon dioxide evolution rates CER during the first five hours of the two B2 processes operated according to the original protocol (triangles and circles, respectively) compared with six other B2 processes at laboratory scale. 
The end of the batch phase during B2 processes was not only indicated by a steep rise of the dissolved oxygen, but also by a sharp drop of the carbon dioxide evolution rate due to the complete consumption of all carbon sources. Approximately one hour before this point, smaller oscillations of the profiles indicated shifts of the cellular metabolism, most likely caused by the transition from high to low glucose concentrations, for the use of alternative carbon sources, like generated byproducts. The courses of the carbon dioxide evolution rates during the first five hours of several different B2 processes, presented in Figure 4-6, were very reproducible with batch phases ending after four to five hours during all cultivations with the exception of the process with the mentioned delay of the feed start, represented with bold lines and circles. The extended batch phase, which was most likely caused by an improper handling of the seed culture prior to inoculation of this process, was the only significant irregularity during this cultivation. However, the cell concentrations after 20 hours as well as the final riboflavin titers were the same as during the other experiment (thin lines and triangles), demonstrating that the overall performance of this $B$. subtilis strain was not critically affected by an extended lag phase.

The delay due to the extended lag phase was reflected in the courses of all crucial process parameters and concentrations of the respective process. However, other than that the process outcome was not impaired. Therefore, the performance of this cultivation could be corrected by subtracting the postponement of four hours from the process time in the respective profiles. Making use of this correction, the cultivation presented with bold lines and circles was considered representative for B2 processes carried out with continuous feeding according to the original protocol. It was designated the reference for the following experiments.

The performance of this reference was compared with a standard B2 process at DSM NP with respect to oxygen uptake rates, which were essentially equivalent (Figure 4-7), growth and riboflavin production (Figure 4-8 A and B, respectively).

The dramatic changes in the course of the oxygen uptake rates at approximately 6 hours and at 15-16 hours process time may indicate major shifts of the cellular metabolism during these times. The first one was most likely linked to the transition of the culture from high glucose conditions, under which by-products like acetoin and acetate were generated, to limited glucose conditions, under which these by-products were consumed again. A transcriptome analysis of $B$. subtilis during the B2 process at DSM NP revealed that the transcription of enzymes for the overflow metabolism (e.g. aceto-lactate-synthase) ceased completely after approximately eight hours of the process (Hohmann, 2004). As a result, there was no further generation of overflow metabolites after twelve hours process time. The concentration of accumulated acetoin, the major by-product of the overflow me- 
tabolism of this $B$. subtilis strain, reached maximum values of almost $5 \mathrm{~g} \mathrm{~L}^{-1}$ approximately 1.5 hours after the start of the feeding phase, and was still detectable until approximately 18 hours process time during B2 processes at DSM NP (data not shown). Therefore, the second peak of the oxygen uptake rates could most likely be attributed to the change from a mixed metabolism of $B$. subtilis, using glucose, acetoin and possibly further by-products as substrates, to metabolizing glucose as the only carbon source.

The initial accumulation of acetoin, which was not considered for the determination of the amount of outgoing carbon $C_{\text {out }}$, may account for total carbon balances clearly below $90 \%$ during the first twelve hours of the processes.

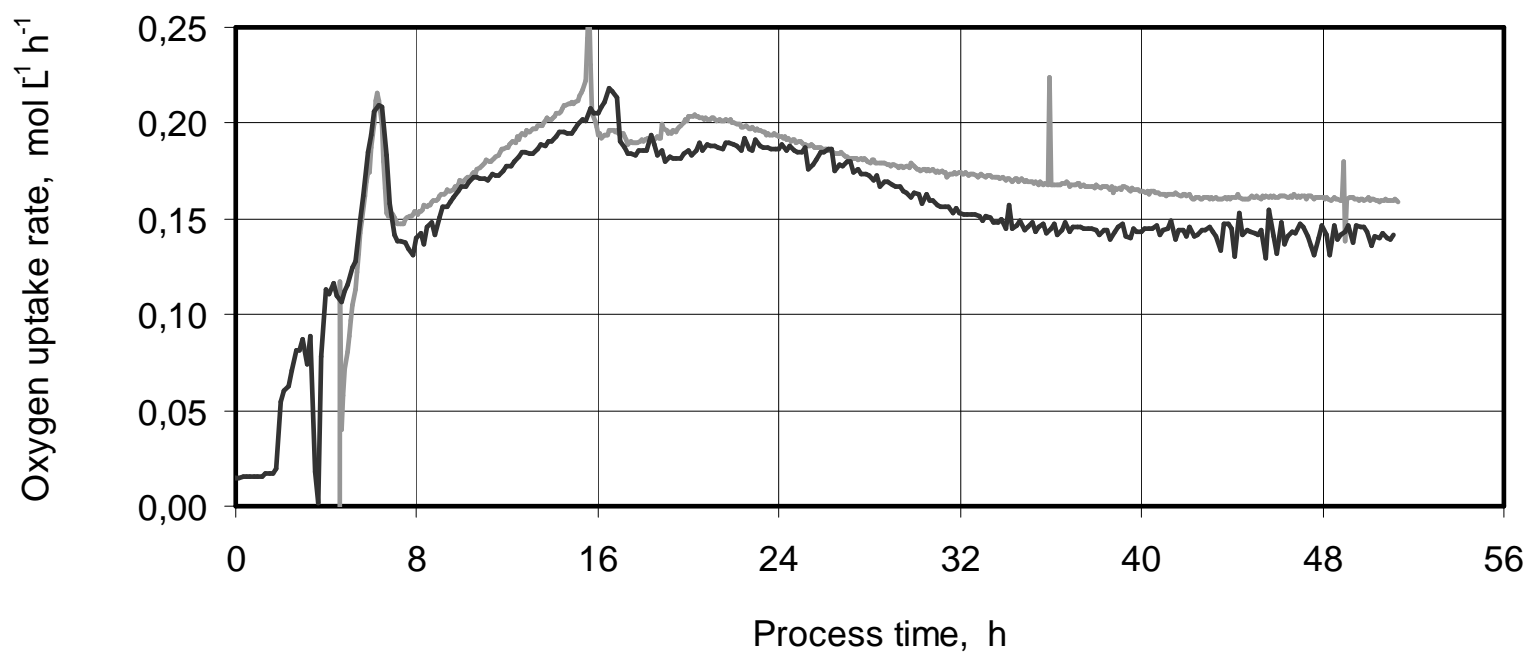

Figure 4-7: Oxygen uptake rates during the selected reference process (dark line) or during the standard B2 process at DSM NP (grey line), both carried out essentially according to the original B2 protocol.

The slow decline of the oxygen uptake rates after 20 hours process time, despite constant or even growing cell concentration, indicated an overall decreasing metabolic activity of the cell population.

The final dry cell mass of $B$. subtilis during the reference process was approximately $45 \mathrm{~g} \mathrm{~L}^{-1}$, and $50 \mathrm{~g} \mathrm{~L}^{-1}$ during the other process in the same setup. This was $13 \%$ or $4 \%$ lower than the final dry cell mass of $52 \mathrm{~g} \mathrm{~L}^{-1}$ during the standard B2 process at DSM NP (Figure 4-8 A). After 24 hours, however, the values during all the processes were in the same range $\left(35-40 \mathrm{~g} \mathrm{~L}^{-1}\right)$. The reason for the different growth behavior during the second half of the process remained unclear.

The profiles for the generated riboflavin concentrations were very similar, yielding 13-14 $\mathrm{g} \mathrm{L}^{-1}$ final concentration (Figure 4-8 B). During the standard B2 process at DSM NP, B. subtilis generated a maximum riboflavin concentration of $13.6 \mathrm{~g} \mathrm{~L}^{-1}$ according to online HPLC measurements. The initial values during this process 
were supposedly too high, most likely due to residual riboflavin in the online HPLC system (communications with DSM NP). The real profile during the first 24 hours was expected to be closer to that during the other two cultivations. The start of the major riboflavin productivity after the completion of the batch phase (Figure 4-3) was probably linked to the transition from overflow metabolism in $B$. subtilis to the metabolic behavior under strict glucose-limited conditions.
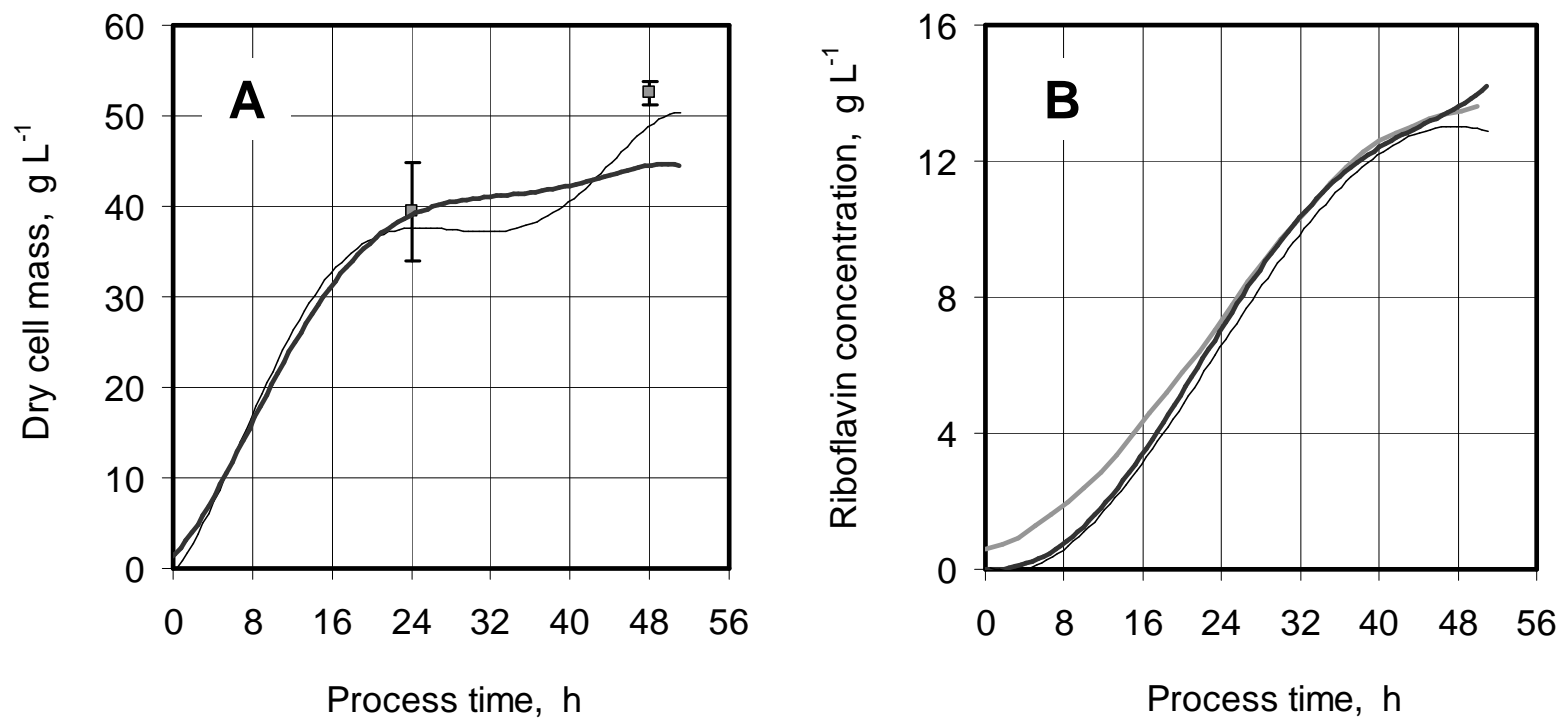

Figure 4-8: Growth and riboflavin production during the reference B2 process at laboratory scale (bold dark lines) and the other process in the same setup (thin dark lines), represented by polynomial regression curves based on the offline raw data of the respective process and compared with data from DSM NP (grey symbols for gravimetric dry cell mass; bold grey line for riboflavin concentration based on online HPLC).

Zamboni et al. (2003) reported riboflavin concentrations equivalent to the presented values during the first 30 hours of a cultivation with a similar fed-batch protocol and the same $B$. subtilis strain. Meanwhile they observed clearly lower biomass concentrations of 17-25 g L $\mathrm{g}^{-1}$ between 20 hours and 30 hours. However, their values were not based on gravimetric determination, but on optical density.

The maximum specific growth and glucose uptake rates were in the expected range. Fischer and Sauer (2005) reported $0.76-1.8 \mathrm{~g} \mathrm{~g}^{-1} \mathrm{~h}^{-1}$ for the specific glucose uptake rates of various exponentially growing Bacillus subtilis clones.

In conclusion, the outcome of the B2 cultivations at laboratory scale was equivalent to a standard B2 process at DSM NP considering the limits of accuracy of biological processes. Therefore, the selected process could serve as a valid reference during the adaptation of the B2 process to the conditions at milliliter scale. 


\subsection{Considerations for the transfer to the milliliter setup}

Table 4-1 compares the stirred tank reactors in the 48-fold parallel setup at milliliter scale with the standard stirred tank reactor at laboratory scale under the conditions that were used during this project. The differences between the two setups, and the required changes of the process operation during scale-down, are explained and discussed in the subsequent paragraphs. They form the basis for the overall concept of this work.

Table 4-1: $\quad$ Comparison of stirred tank bioreactors at milliliter scale and at laboratory scale. Abbreviations: $D O$, dissolved oxygen; $T$, temperature; $\mathrm{O}_{2}$, oxygen concentration; $\mathrm{CO}_{2}$, carbon dioxide concentration; $O D$, optical density; $c_{X}$, dry cell mass; $c_{S}$, substrate (glucose) concentration; $c_{P}$, product (riboflavin) concentration.

\begin{tabular}{lcc}
\hline & Laboratory scale & Milliliter scale \\
\hline Vessel & conventional stirred tank & baffled polystyrene vessels \\
Initial volume & $3 \mathrm{~L}$ & $10 \mathrm{~mL}$ \\
Parallel reactors & 1 & 48 \\
Agitator & $3 \times$ Rushton turbine & magnetic gas-inducing turbines \\
Stirrer speed & $400-1500$ rpm; $D O$-controlled & 2200 - 2700 rpm; constant \\
Aeration & sparger & gas-induction \\
Increased oxygen & excess pressure & oxygen-enriched gas flow \\
input & continuous & intermittent \\
Feeding & continuously online & every 30 min at-line \\
$p H$ measurement & quasi-continuous & intermittent \\
$p H$ control & closed loop control & customized profile \\
Antifoam addition & $D O, T$, exhaust gas $\left(O_{2}, C O_{2}\right)$ & online: $D O(8 x)$ \\
Process monitoring & online: & automated at-line: $O D, c_{S}, C_{P}$ \\
\hline
\end{tabular}

The setup at milliliter scale did not include a technique for the continuous addition of liquids to the parallel reactors. Instead, liquids were added discontinuously by a laboratory robot, which was equipped with eight individually controllable pipettes. Consequently, one of the most fundamental operational modifications was the shift of the feeding mode from continuous to intermittent. The time between each substrate addition had to be as short as possible for the realization of a quasicontinuous feeding mode. At the same time, the intervals had to be long enough to allow sampling and dilution activities throughout the process.

The discontinuous liquid addition also involved the provision of base for $\mathrm{pH}$ adjustment during the process, which was quasi-continuous at laboratory scale (additions once every minute), but also had to be intermittent in the milliliter setup. 
Parallel online $\mathrm{pH}$ measurement inside the milliliter bioreactors was not possible. Hence the $\mathrm{pH}$ sensing could only take place outside of the vessels at-line, which additionally hampered the $\mathrm{pH}$ control due to the limited availability of $\mathrm{pH}$ data. The envisioned frequency for $\mathrm{pH}$ measurements was once every $30 \mathrm{~min}$.

Controlling the stirrer speed, by using online signals of the dissolved oxygen, was not intended at the milliliter scale. Therefore, a constant agitation value had to be used for the whole process. According to the original protocol for the B2 process, the aeration of the stirred tank reactor at laboratory scale took place with air. This required the application of excess pressure to the system, to generate an oxygen input which was sufficiently high for optimal riboflavin production. This indicated that an enhancement of the oxygen transfer would also be necessary at the milliliter scale, despite the fact that the gas-inducing turbines could generate higher volumetric oxygen transfer coefficients compared to the standard stirred tank at laboratory scale (Puskeiler et al., 2005a). Since the application of excess pressure to the 48 vessels at milliliter scale was not feasible, an alternative strategy for realizing higher oxygen transfer rates was to enrich the gas flow with oxygen.

A further aspect during the adaptation of the B2 process to the milliliter scale was the handling of foam formation, which could not be detected automatically in the miniature vessels. Hence, the addition of a foam suppressing agent had to take place according to a customized profile instead of being triggered on demand which was the case at laboratory scale.

None of the described changes in operation should influence the performance of the B2 process, particularly with respect to the riboflavin production. Therefore, the process conditions were altered one step at a time, starting from the reference protocol, and were simulated in the standard stirred tank reactor due to better monitoring opportunities at this scale. The individual effects were investigated thoroughly before the modified B2 process was run at the milliliter scale. 


\subsection{Investigations at laboratory scale}

This section describes several steps during the adaptation of the B2 process to the conditions at the milliliter scale. The first modification was the shift from continuous to intermittent feeding at laboratory scale. All other conditions remained unchanged. The next change involved carrying out the process at ambient pressure, using oxygen-enriched air for aeration. Finally, also the base addition and $\mathrm{pH}$ control at laboratory scale were carried out intermittently. At the end of this section, a summary comprises the major outcome of these investigations and the consequences for the operation of the B2 process in the milliliter setup.

\subsubsection{Effect of intermittent feeding during B2 processes}

The goal of the intermittent feeding mode at laboratory scale was to simulate the feed addition of the laboratory robot in the milliliter setup. This was achieved by keeping the time required for the actual addition of the substrate solution to a minimum (in the range of seconds) at the beginning of a feeding interval, which lasted several minutes.

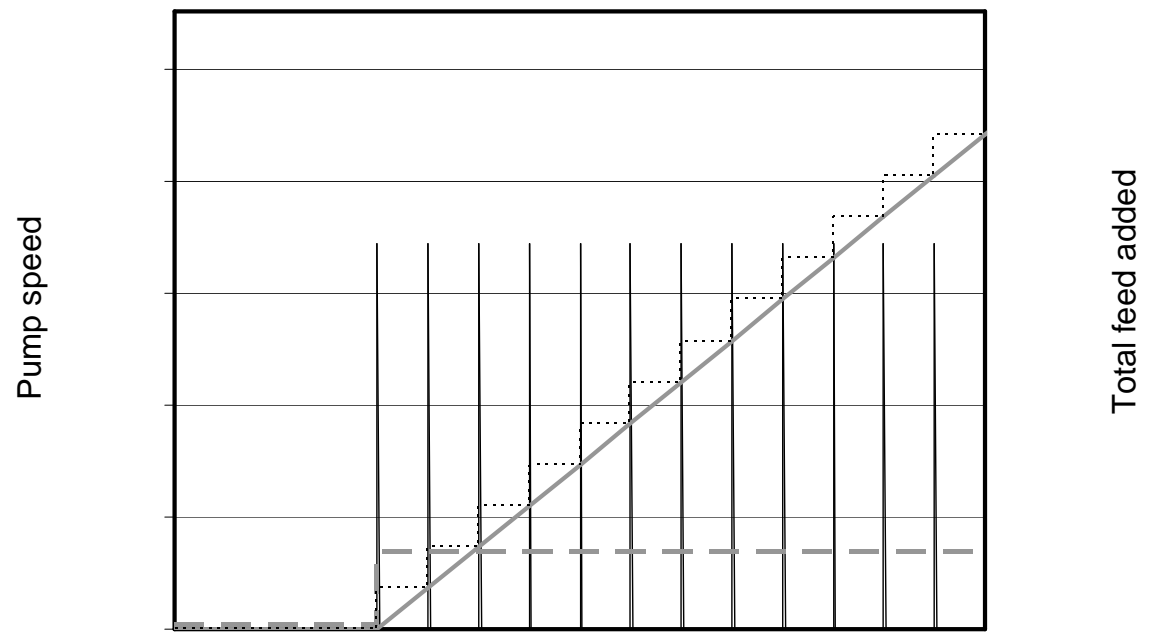

Process time

Figure 4-9: Principle of the intermittent feed addition during fed-batch cultivations. The solid peaks after the initial batch phase represent the feeding pulses. Using this procedure the same amount of feed is added as it would be during a continuously fed process (constant pump speed; dashed grey line). The amount of added feed increases incrementally for the intermittent mode (dotted line) and continuously for the standard feeding procedure (solid grey line). (Note: For a more concise illustration, the graphic does not display the real relation between the pump speeds. The intermittent speed has to be larger approximately by a factor of 60 in order to provide the necessary amount of feed for the described procedure.) 
The amount of nutrients added during one feed pulse was equivalent to the amount of nutrients added during the same time in a continuously fed experiment. Hence the overall substrate addition to a process was the same for either feeding mode. The described concept is demonstrated in Figure 4-9. For a longer feeding interval a larger feed volume had to be injected with each feed pulse, yielding a higher initial glucose concentration in the medium right after the addition.
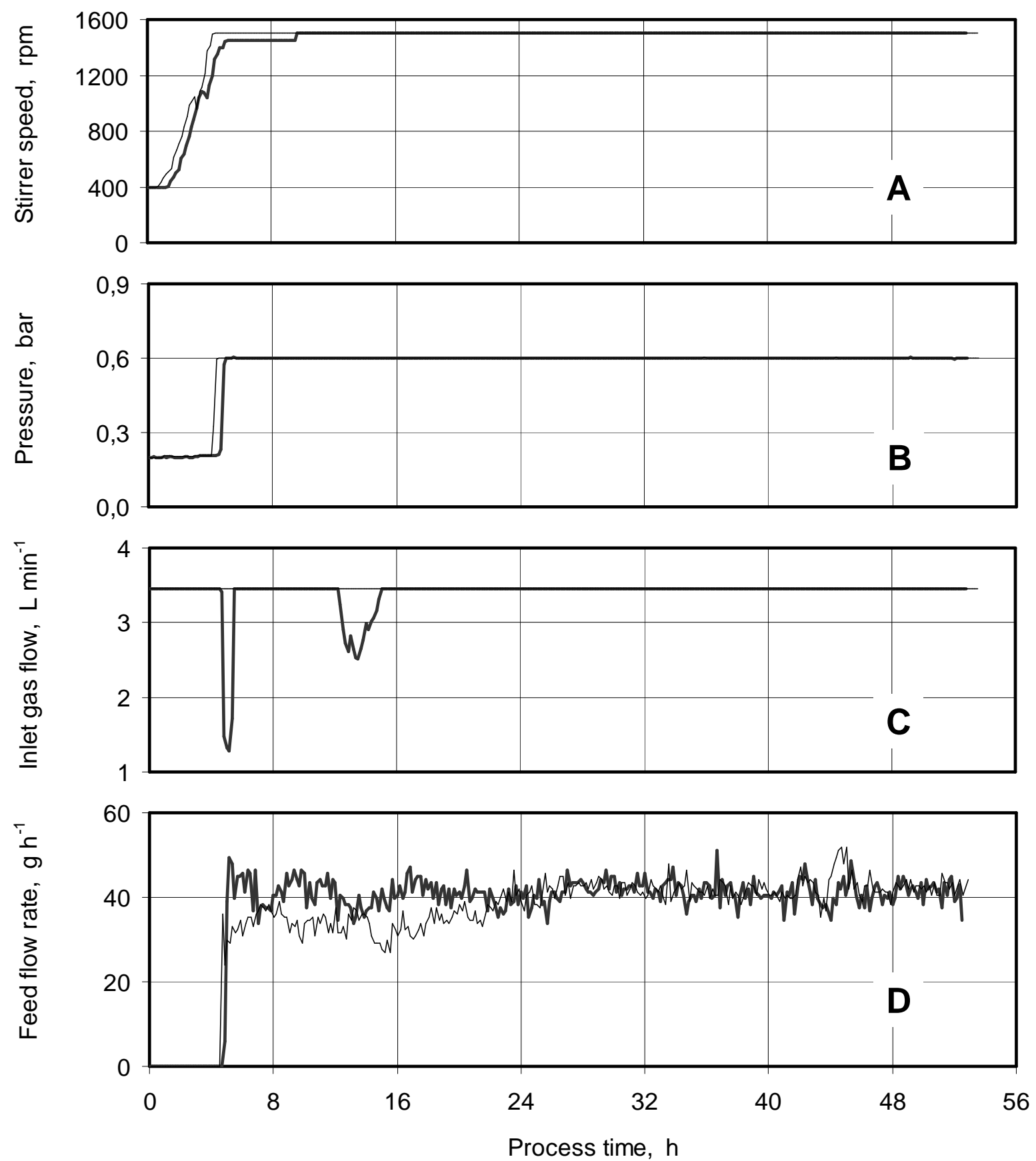

Figure 4-10: Stirrer speed (A), pressure (B), inlet gas flow (C), and feed addition (D) during two B2 processes at laboratory scale, carried out according to the original protocol except for feeding which was intermittent every $5 \mathrm{~min}$. Graphs for the feed flow rate are based on the feed consumption according to the feed balance. 
A feeding interval of 5 min was applied for the intermittent fed-batch operation of several B2 processes at otherwise unchanged process operation compared to the original protocol (Figure 4-10). This was except for the stirrer speed which was set to a constant value of $1500 \mathrm{rpm}$ at the end of the batch phase (Figure 4-10 A). The discontinuous feed addition was achieved with a peristaltic pump which could be controlled by the process control software. The feed pulses lasted five or seven seconds, depending on two different pumps used in the experiments. This corresponded to a ratio of the feed pulse duration to the length of the feeding interval between each pulse of $1: 60$ or $1: 43$, respectively. During one process (thin lines), the feed provision was insufficient until 22 hours process time (Figure 4-10 D). During the other process, a process downtime between 5.4 and 5.8 hours and problems with the gas flow control between twelve and 15 hours resulted in periods of reduced inlet gas flow (Figure 4-10 C).
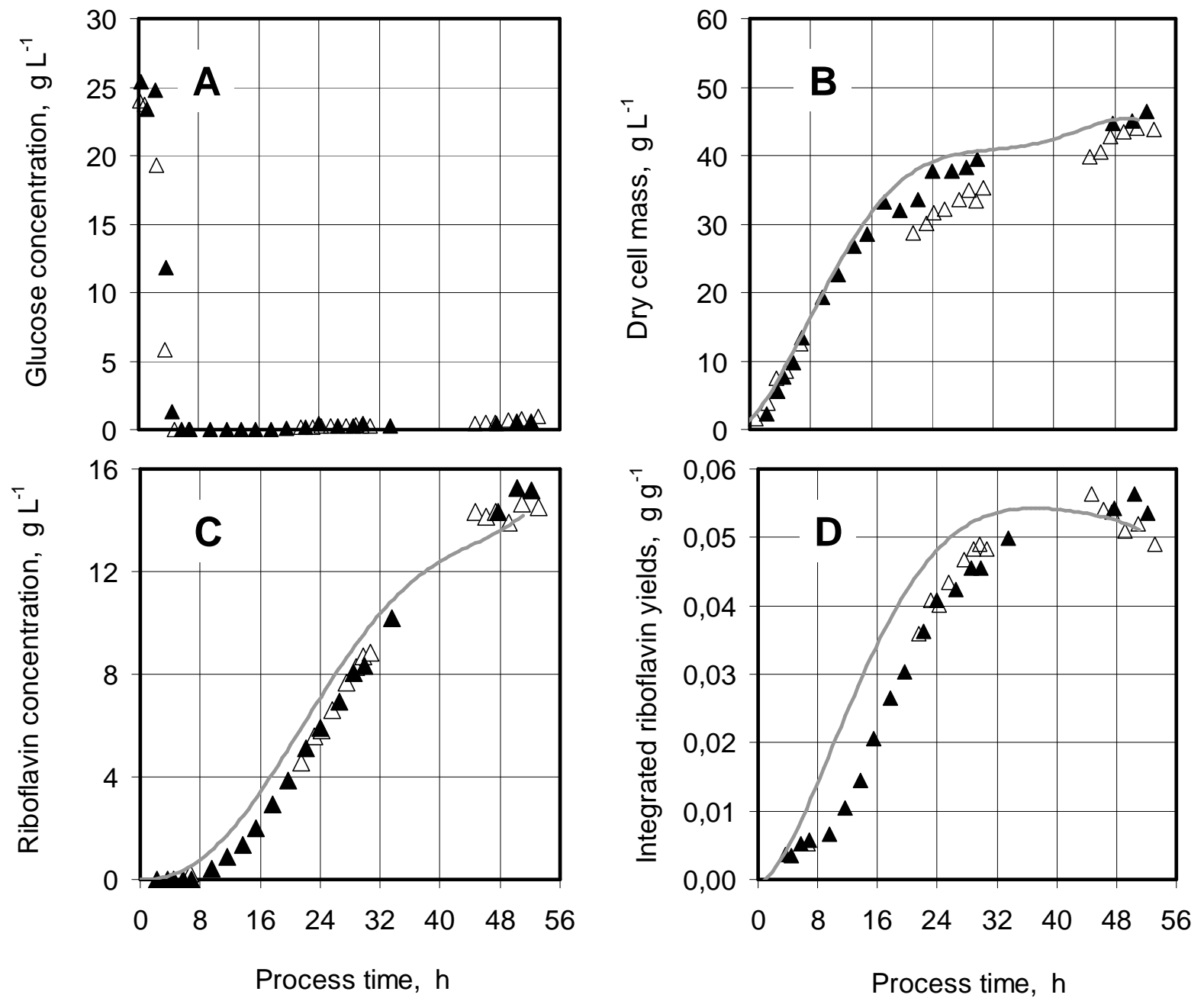

Figure 4-11: Glucose consumption (A), growth (B), riboflavin concentration $(C)$ and integrated product yields (D) generated by $B$. subtilis based on the offline analysis during two B2 processes at laboratory scale, operated with intermittent feeding every 5 min (open or solid triangles, respectively). Dry cell mass values are based on gravimetric determination. Riboflavin concentrations are based on the average of HPLC and absorbance measurements. The grey lines represent the continuously fed reference. 
The resulting glucose, dry cell mass and riboflavin concentrations for the two processes are displayed in Figure 4-11 as open and solid triangles, respectively. The batch phases during both cultivations lasted four to five hours (Figure 4-11 A). The dry cell mass concentrations revealed that the growth of $B$. subtilis during the cultivation represented with solid triangles was not significantly influenced by the examined intermittent feeding interval compared to the continuously fed reference, whereas the dry cell mass concentrations during the second day of the other cultivation were clearly lower, but reached the reference values at the end of the process.
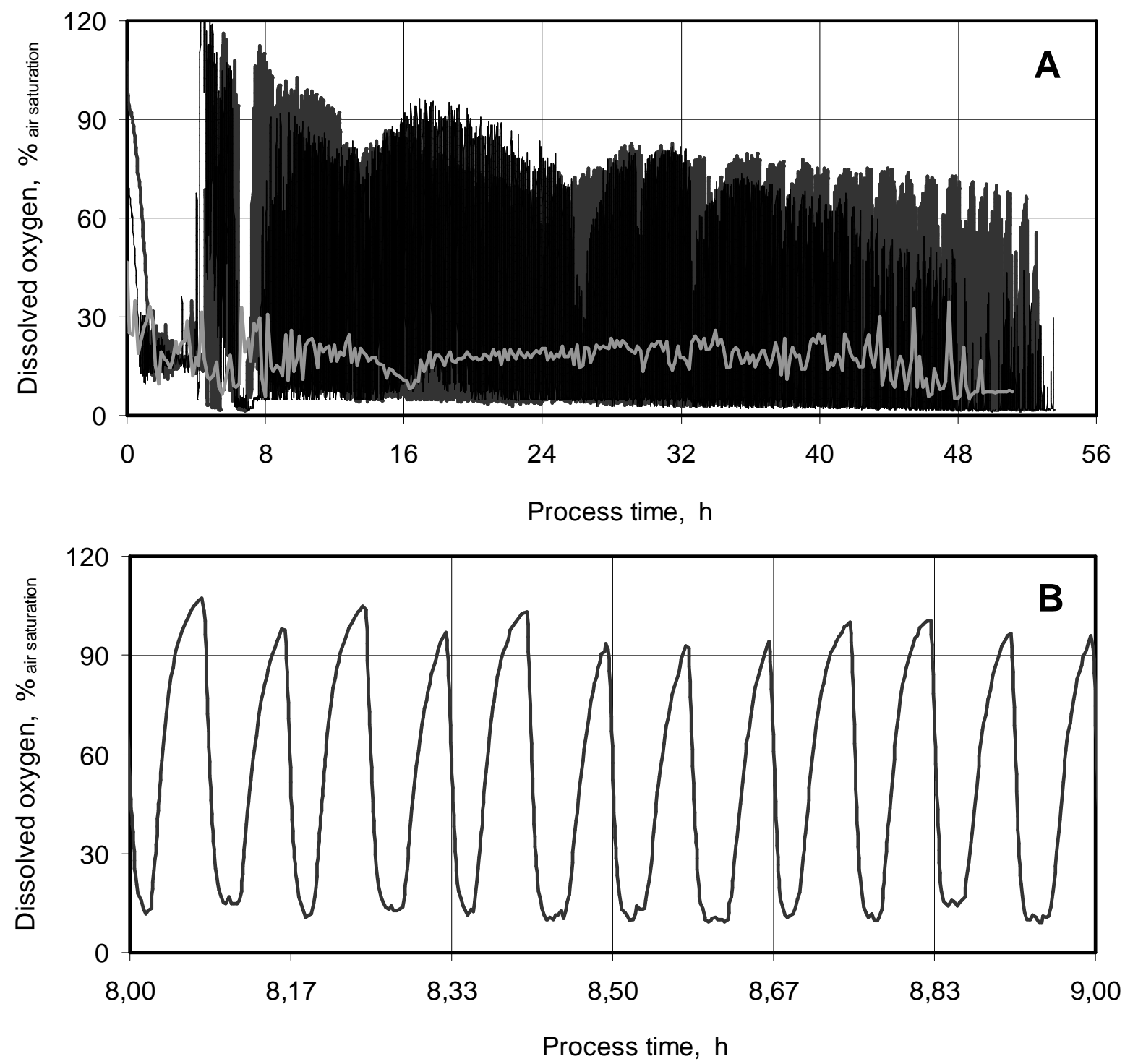

Figure 4-12: A: Fluctuating dissolved oxygen profiles for two B2 processes at laboratory scale, operated with intermittent feeding every 5 min (dark lines), compared to the continuously fed reference process (grey line). B: Period of one hour during one of the intermittent processes. 
The final riboflavin concentrations during the intermittently fed processes were slightly higher than the continuously fed reference with $14-15 \mathrm{~g} \mathrm{~L}^{-1}$ compared to 13-14 $\mathrm{g} \mathrm{L}^{-1}$, respectively (Figure 4-11 C). However, the riboflavin production started delayed by approximately three hours compared to the continuously fed reference, based on the riboflavin concentrations, or even five to six hours delayed according to the integrated product yields (Figure 4-11 D). The final yields were $0.050-0.056 \mathrm{~g} \mathrm{~g}^{-1}$, corresponding to the reference.

The pulsed provision of glucose to the system resulted in characteristic fluctuations of the dissolved oxygen, which dropped dramatically right after each substrate addition, even down to concentrations below $5 \%$ air saturation, and rose steeply a few minutes later upon consumption of the carbon sources. This is shown in Figure 4-12. The values above $100 \%$ result from the increase of excess pressure to 600 mbar upon start of the feeding phase. The drop of the dissolved oxygen at six to seven hours process time indicated insufficient oxygen transfer rates during this period.

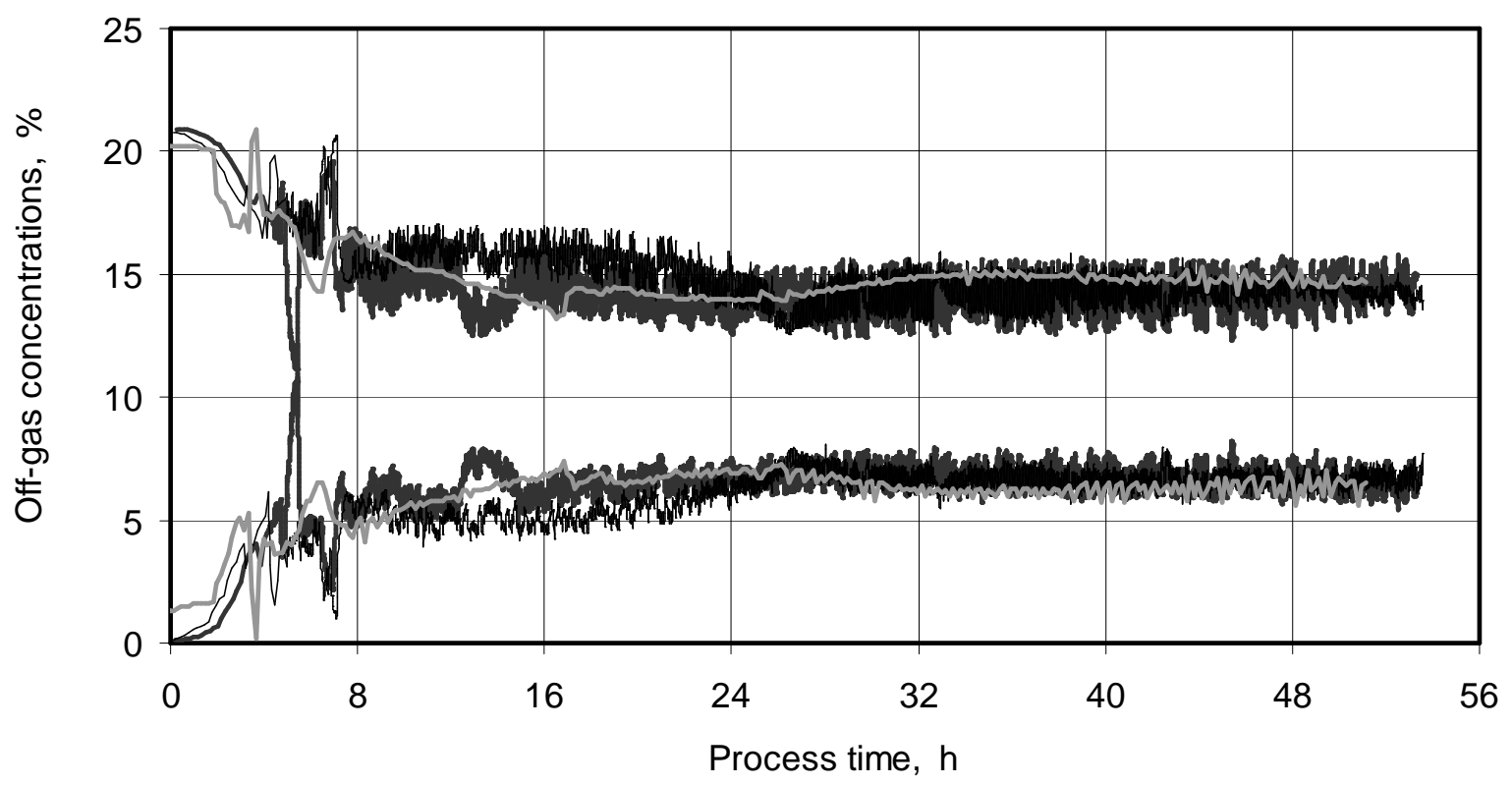

Figure 4-13: Fluctuating oxygen concentrations (upper curves) and carbon dioxide concentrations (lower curves) in the off-gas of two B2 processes at laboratory scale, operated with intermittent feeding every 5 min (dark lines). The data are compared with off-gas concentrations during the continuously fed reference process (grey lines).

Fluctuations also occurred in the off-gas concentrations of oxygen and carbon dioxide (Figure 4-13). The average course of the respective profiles essentially followed the courses of the reference process. The only major difference was the increase or drop in oxygen or carbon dioxide concentration, respectively, between six and seven hours process time, which was opposite to the behavior of the reference profile. Irregular behavior of the intermittent process represented with 
bold dark lines at 5.4 hours and between 12.5 hours and 15 hours in both of the determined off-gas concentrations could be attributed to the reduced inlet gas flow during these times (Figure 4-10 C). The reason for the lower carbon dioxide and higher oxygen concentrations between eight and 22 hours during the other process was the inferior feeding rate (Figure 4-10 D).

The insufficient feeding rate was also reflected in the course of the oxygen uptake rate during this process (thin black line in Figure 4-14), which was lower than during the other intermittent process by approximately $40 \mathrm{mmol} \mathrm{L}^{-1} \mathrm{~h}^{-1}$ during this period. During the batch phase and after approximately 17 hours process time, the average of the oscillating profiles in Figure 4-14 followed the course of the reference process (grey line). However, the temporary maximum oxygen uptake rates right after each substrate addition during the intermittently fed processes were up to $50 \mathrm{mmol} \mathrm{L}^{-1} \mathrm{~h}^{-1}$ higher than those during the continuously fed experiment. Two hours after the start of the feeding phase until approximately 17 hours process time, the profiles of the intermittent experiments diverged from that of the continuously fed reference.

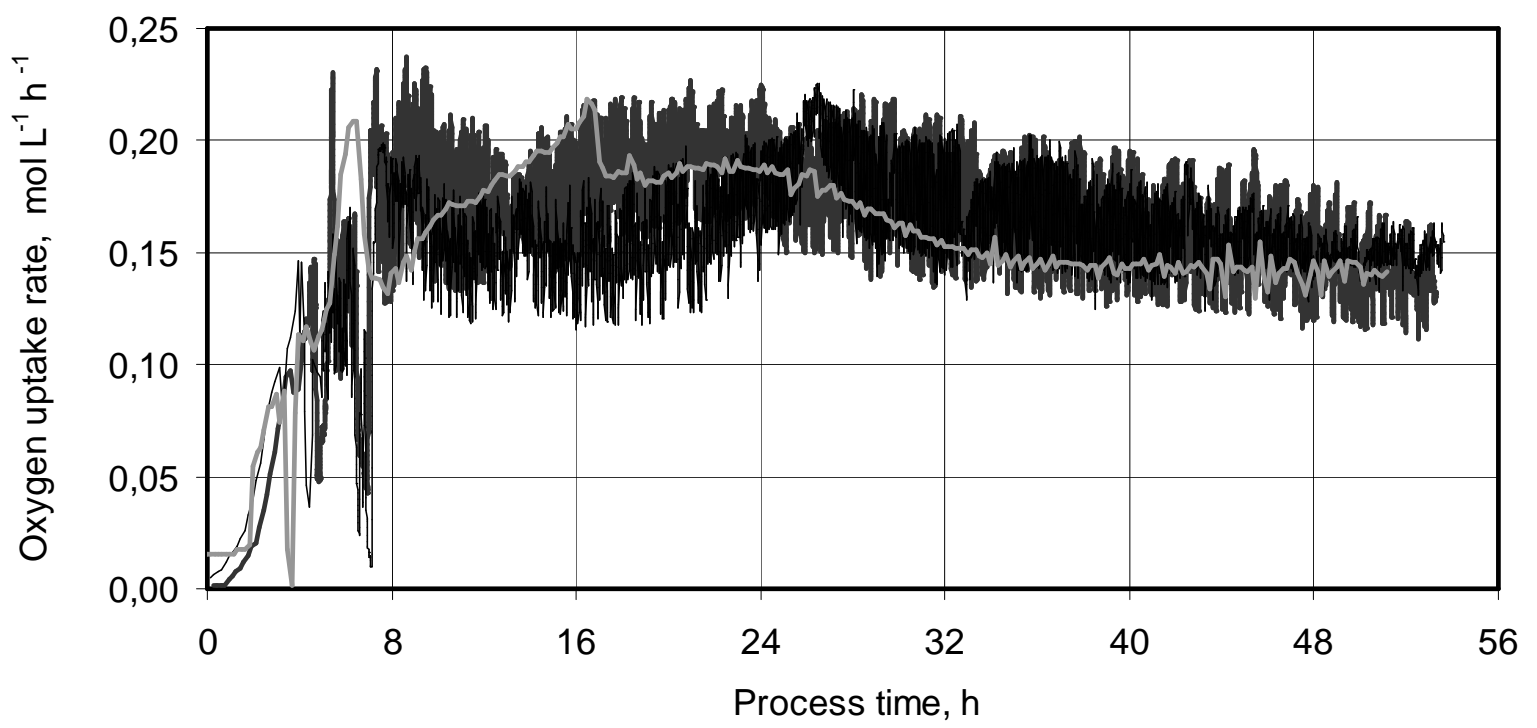

Figure 4-14: Fluctuating oxygen uptake rates during two B2 processes, operated with intermittent feeding every 5 min (dark lines), compared with the continuously fed reference process (grey line).

The total carbon balances for the two processes are shown in Figure 4-15. The values were equivalent to the reference process and indicated the existence of not detected by-products like acetoin until approximately 16 hours process time. Thereafter no significant accumulation of by-products occurred, because the amount of undetected compounds was clearly below $10 \%$. The partial balances for carbon dioxide only, as well as for carbon dioxide and biomass, were also in the same range as the reference (data not shown). 


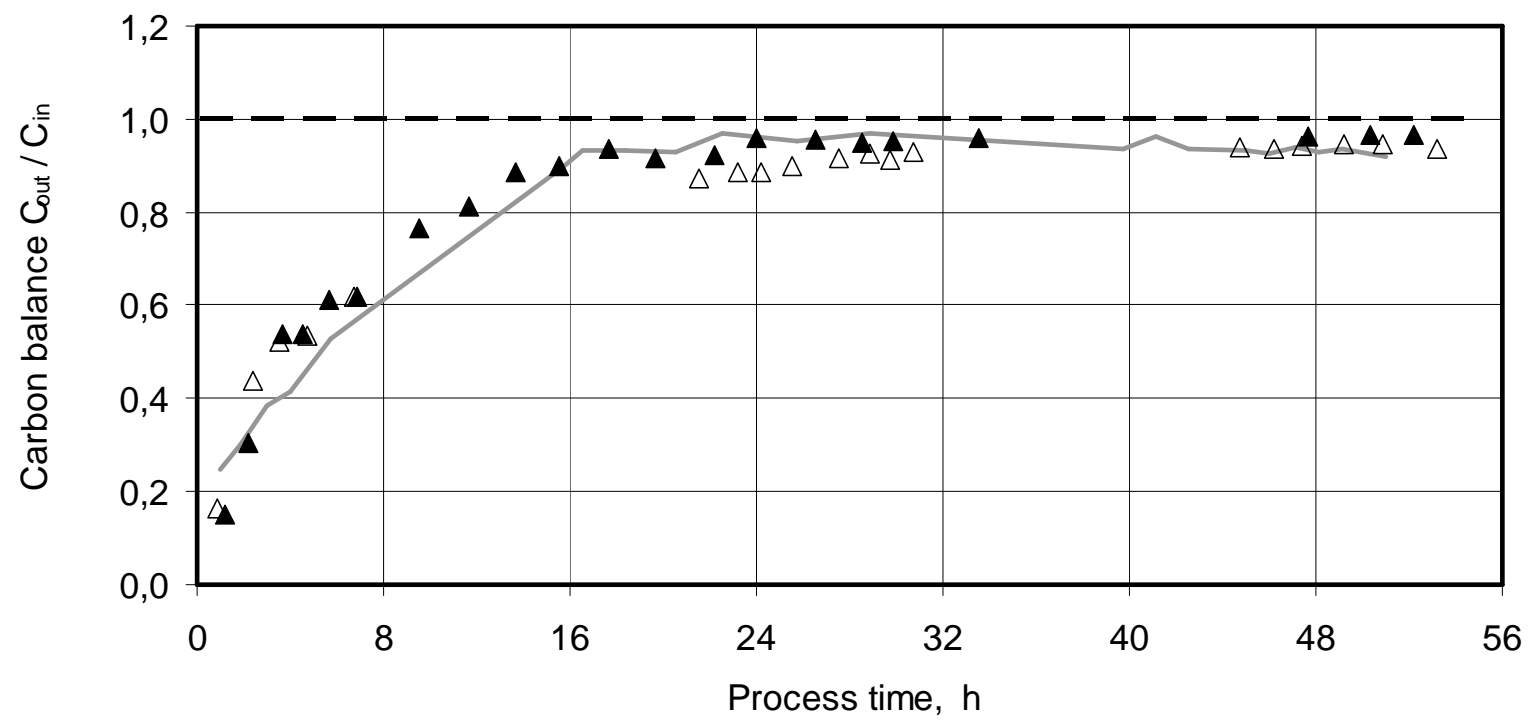

Figure 4-15: Total carbon balances for two B2 process at laboratory scale, operated with intermittent feeding every $5 \mathrm{~min}$ (triangles) compared with the continuously fed reference (grey line).

\section{Discussion}

The performance of B2 processes under these experimental conditions indicated that the growth behavior of $B$. subtilis was not significantly influenced by an intermittent feeding mode with intervals of 5 min when sufficient feed was provided. Also the final riboflavin concentrations were equivalent to the continuously fed reference. However, the onset of the riboflavin production was delayed by several hours compared to the reference. Since the increase of cell mass was not postponed, it may be concluded that this delay was triggered directly by the pulsed substrate addition or indirectly by the temporarily reduced minimum dissolved oxygen concentrations.

The course of the off-gas concentrations between six and seven hours process time during the intermittent experiments indicated lower metabolic activity during this period, given that the gas flow, pressure and feed flow remained constant. This was not in agreement with the low dissolved oxygen concentrations during this time, which indicated maximum oxygen consumption. The reason for this inconsistency of data remained unclear. Increased oxygen uptake rates were more likely, because these were observed for the reference process during the respective process time.

The intermittent feeding mode caused temporarily significant increased oxygen uptake rates, when judged against a continuously fed cultivation. This requires an enhanced provision of oxygen for intermittently operated B2 processes compared to the original protocol. Feeding intervals of $7 \mathrm{~min}$ and $10 \mathrm{~min}$ have been tested during several intermittent B2 processes at similar process conditions (Knepper, 
2003). The oxygen transfer rates during these experiments were not sufficient for the desired riboflavin production, indicating that the observed phenomenon becomes more significant with extended periods between the feed pulses.

The difference of the courses of oxygen uptake rates between the intermittent and the continuously fed B2 processes after approximately six hours process time may have been caused by different ways of the cell populations to switch from overflow metabolism to glucose-limited metabolism due to the dynamic changes of substrate and possibly of by-product concentrations.

\subsubsection{B2 processes at ambient pressure with oxygen addition}

It is not possible to apply excess pressure to the vessels in the milliliter setup. Therefore, the necessary enhancement of the oxygen transfer rate for optimal riboflavin productivity should be achieved by enriching the air flow with oxygen. The fraction of oxygen in the inlet gas flow at ambient conditions ( $p=1.0$ bar) must be approximately $33.5 \%$ in order to yield a partial pressure of oxygen in the medium which is equivalent to the oxygen tension resulting from aeration with pure air at 600 mbar excess pressure. This estimation is based on the proportional increase of the partial pressure with the total pressure (Dalton's Law), and is based on the assumption that the Henry constant for the dissolution of oxygen in the medium is the same in both cases.

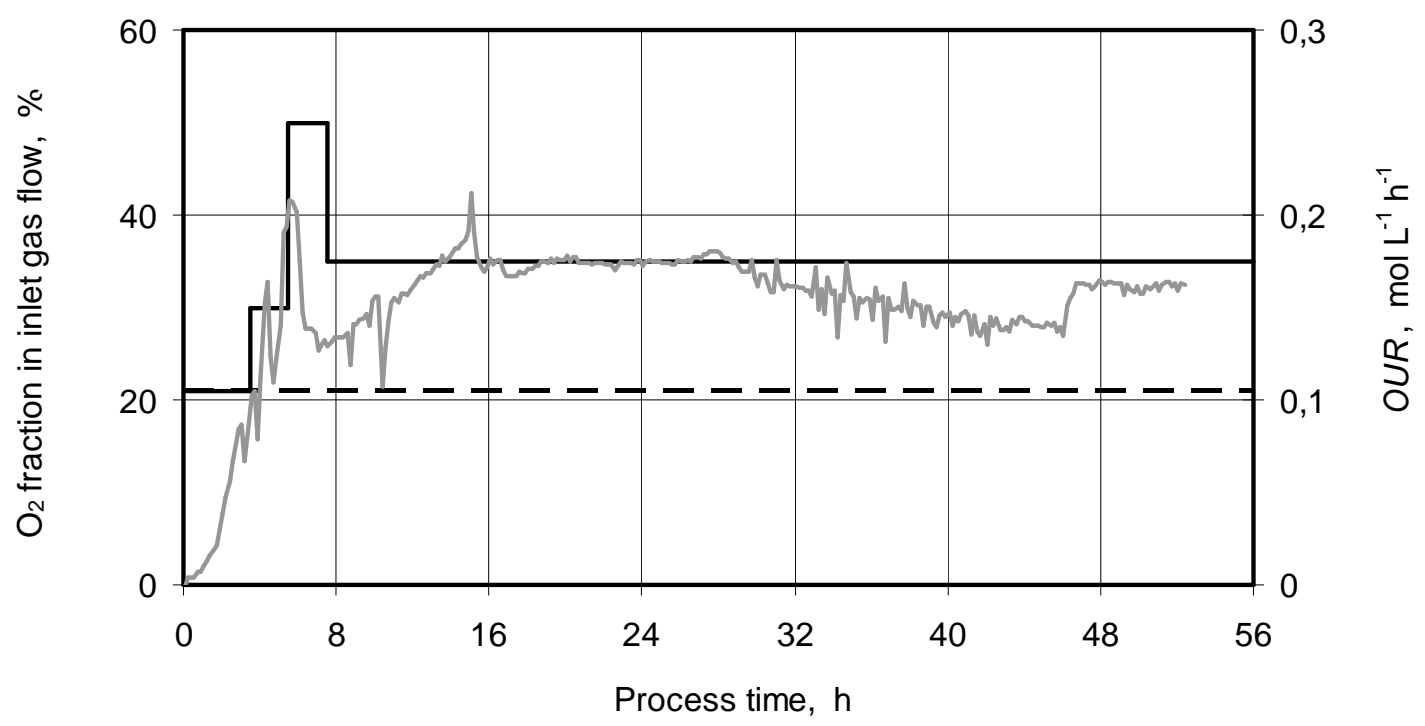

Figure 4-16: Oxygen supply profile for the B2 process at ambient pressure (black line), suggested by the course of the oxygen uptake rate (OUR; grey line) during a reference process with excess pressure and aeration with air only (dashed line).

The oxygen uptake rates during continuously fed B2 processes indicated the oxygen need for successful riboflavin production, which suggested the profile pre- 
sented in Figure 4-16 for the addition of oxygen. The aeration started off with air only ( $20.95 \%$ oxygen content). After 3.5 hours, the oxygen fraction in the inlet gas flow was raised to $30 \%$, and to $50 \%$ after 5.5 hours. It was reduced to $35 \%$ after two more hours for the remaining time of the process.

The impact of this method for the achievement of an increased oxygen supply was initially investigated during a continuously fed B2 process. The only additional difference compared to the original protocol was a constant stirrer speed of $1200 \mathrm{rpm}$. Subsequently, the previously tested intermittent feeding mode was applied, firstly by using intervals of $5 \mathrm{~min}$ between the substrate pulses. These were then extended to 10 min during the following cultivations in order to allow more time for robotic actions between each feed addition in the envisioned milliliter setup.

Growth and riboflavin productivity during the continuously fed B2 process at ambient pressure (black squares in Figure 4-17) were similar to the reference at excess pressure (grey line), yielding approximately $47 \mathrm{~g} \mathrm{~L}^{-1}$ final dry cell mass and $14 \mathrm{~g} \mathrm{~L}^{-1}$ final riboflavin concentration.
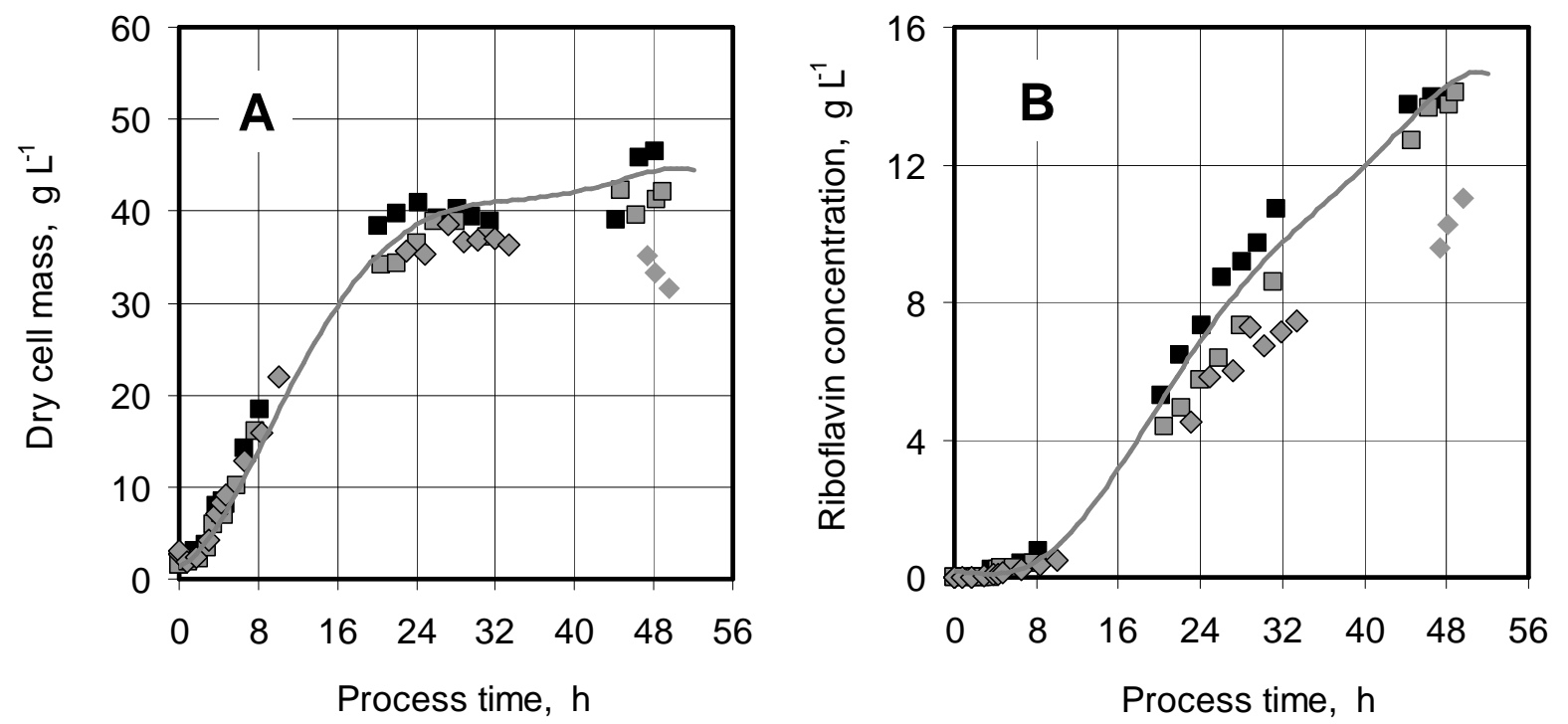

Figure 4-17: Dry cell mass $(\mathrm{A})$ and riboflavin concentrations $(\mathrm{B})$ during $\mathrm{B} 2$ processes at ambient pressure at laboratory scale compared to the reference operated according to the original protocol (grey line). The feeding mode was either continuous (black squares) or intermittent with a $5 \mathrm{~min}$ or $10 \mathrm{~min}$ interval (grey squares or diamonds, respectively). Oxygen addition to the air flow was according to the profile presented in Figure 4-16. The different display of last three values represented with circles indicates a missing $\mathrm{pH}$ control after 36 hours during the respective process.

For a 5 min feeding interval (grey squares in Figure 4-17), growth was not delayed during the first ten hours of the process, but was slightly lower during the second day, before it reached a final dry cell mass concentration of $40-43 \mathrm{~g} \mathrm{~L}^{-1}$. 
The final riboflavin concentration during this experiment was also $14 \mathrm{~g} \mathrm{~L}^{-1}$. However, the onset of the production was approximately four hours later compared to the reference.

Increasing the feeding interval from 5 min to 10 min during the next experiment (grey diamonds in Figure 4-17) resulted in a similar growth during the first 32 hours and a similar delay of the riboflavin production. After 30 hours the riboflavin concentrations remained clearly below the values of the other cultivations. After 36 hours during this process, the $\mathrm{pH}$ could not be kept at its setpoint, but decreased to 5.1 until the end of the process, due to a damaged base tubing which started leaking outside the reactor. Since this most likely had an impact on the subsequent results of the riboflavin concentration and of the dry cell mass, the last three analytical values of this process in Figure 4-17 are displayed as grey symbols without the black frame.

\section{Discussion}

The provision of additional oxygen in the gas flow, with the goal of increasing the oxygen transfer during the B2 process, seems to be a feasible way to substitute the application of excess pressure to the reactor. The employed oxygen concentrations allowed the reproduction of the reference process when the feed addition was continuous. However, during intermittent experiments, the riboflavin production was delayed by a few hours where the process involved pulsed addition of substrate every $5 \mathrm{~min}$, and even more so where the process involved a $10 \mathrm{~min}$ feeding interval. During the latter the values around the 30 hour time frame indicated that most likely the final product concentration of the reference could not be reached. During the intermittent experiments, also the dry cell mass concentrations were a little delayed after 20 hours and slightly lower compared to the continuously fed reference with oxygen addition.

The results of these experiments imply again that the intermittent feeding has an impact on the performance of $B$. subtilis, which gets more pronounced for the longer feeding intervals. In this case, the provision of oxygen was not sufficient for a reproduction of the riboflavin concentrations of the reference. This outcome underlines that the generation of riboflavin by $B$. subtilis depends strongly on a sufficiently high provision with oxygen. Due to the temporarily significantly higher oxygen uptake rates during intermittently fed B2 processes, increased oxygen transfer rates are required compared to continuously fed cultivations, particularly with lengthening of the feeding intervals. Therefore, even higher oxygen fractions in the inlet gas flow should be employed. 


\subsubsection{B2 processes with intermittent feeding and $\mathrm{pH}$ control}

In the milliliter setup, the $\mathrm{pH}$ could not be measured online inside the vessels but had to be determined at-line outside of the vessels. During the original B2 process at laboratory scale, ammonium hydroxide $\left(\mathrm{NH}_{4} \mathrm{OH}\right)$ was provided quasicontinuously on demand according to a PID control provided with the process control software. During the following experiments, all of which were carried out with a 10 min feeding interval at ambient pressure, the PID control was replaced with an intermittent $\mathrm{pH}$ control, which was restricted to using $\mathrm{pH}$ values only once every 30 min and which triggered the addition of base every 5 min or $10 \mathrm{~min}$.

Oxygen was added to the gas flow, initially according to the profile presented in Figure 4-16 for sufficient oxygen transfer rates at ambient pressure conditions. However, during the cultivation with a 5 min interval for base addition the outlet gas filter was stuck, resulting in excess pressure of 350-450 mbar after 10 hours until the end of the process, and thus in respectively higher dissolved oxygen compared to conditions at ambient pressure (data not shown). During the experiment with base addition every $10 \mathrm{~min}$, the oxygen fraction was not lowered to $35 \%$ after 7.5 hours, but was kept at $50 \%$ until the end of the processes.
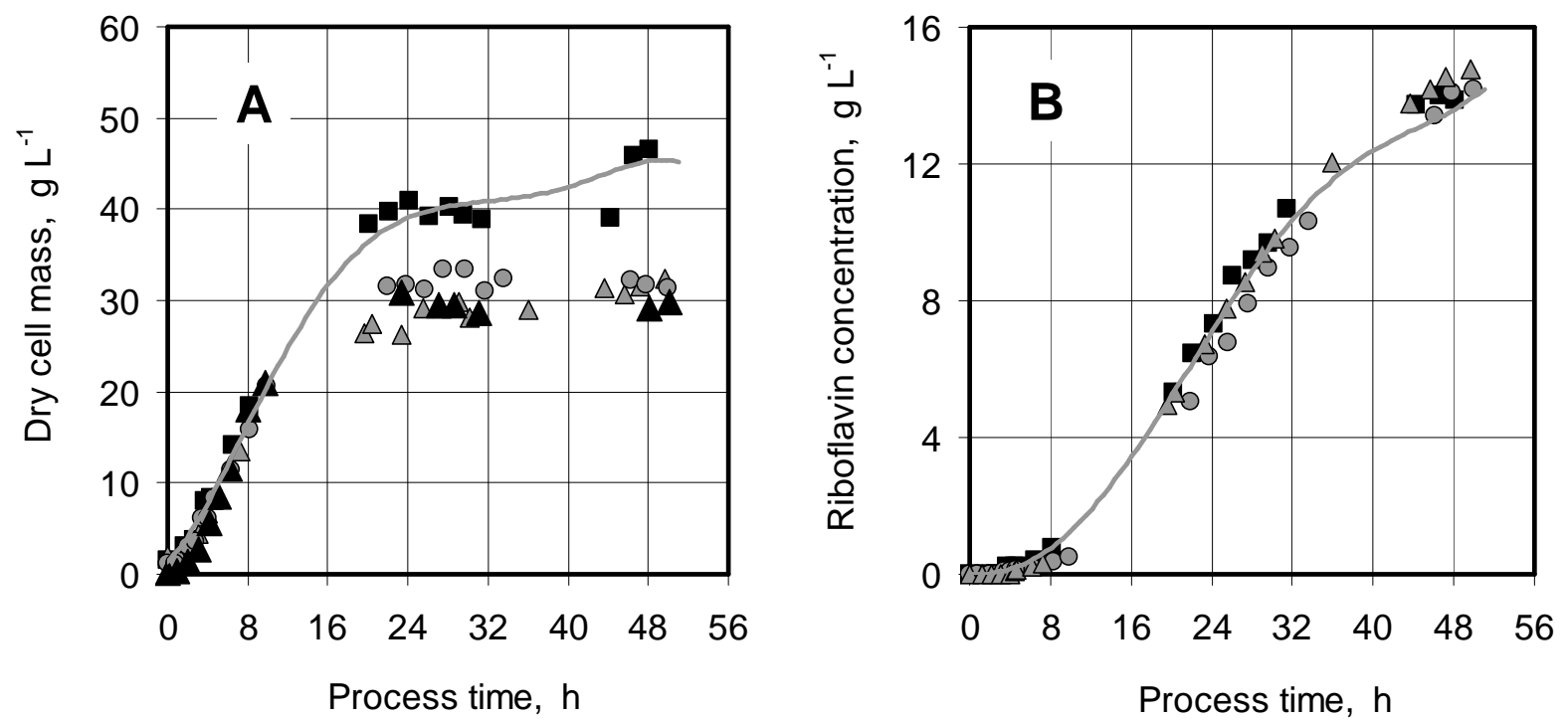

Figure 4-18: Dry cell mass (A) and riboflavin concentrations (B) during two intermittent $B 2$ processes at ambient pressure with oxygen addition (grey symbols). They received feed every $10 \mathrm{~min}$ and base was added every 5 or $10 \mathrm{~min}$ (triangles or circles, respectively). They are compared with the reference operated according to the original B2 protocol (grey line) and with two continuously fed processes at ambient pressure (black symbols), of which one was operated with $35 \%$ oxygen in the gas flow during the feeding phase (black squares), the other one with $50 \%$ (black triangles).

During these intermittent cultivations the cell mass generation was considerably lower than during the reference process or during the continuously fed B2 process at ambient pressure, yielding final dry cell mass concentrations of only $30-32 \mathrm{~g} \mathrm{~L}^{-1}$ 
compared to $45 \mathrm{~g} \mathrm{~L}^{-1}$ (grey symbols compared to the grey line or to black squares in Figure 4-18 A, respectively). However, this phenomenon was also observed for a continuously fed B2 process which was aerated with $50 \%$ oxygen content in the gas flow from the beginning of the feeding phase (black triangles).

The riboflavin concentrations during the intermittent experiments reached the reference values (Figure 4-18 B). During the process with 10 min intervals for base and feed addition and without excess pressure (grey circles), which was the operation envisioned for the final protocol in the milliliter setup, the onset of the riboflavin production seemed slightly delayed compared to the other successful B2 processes.
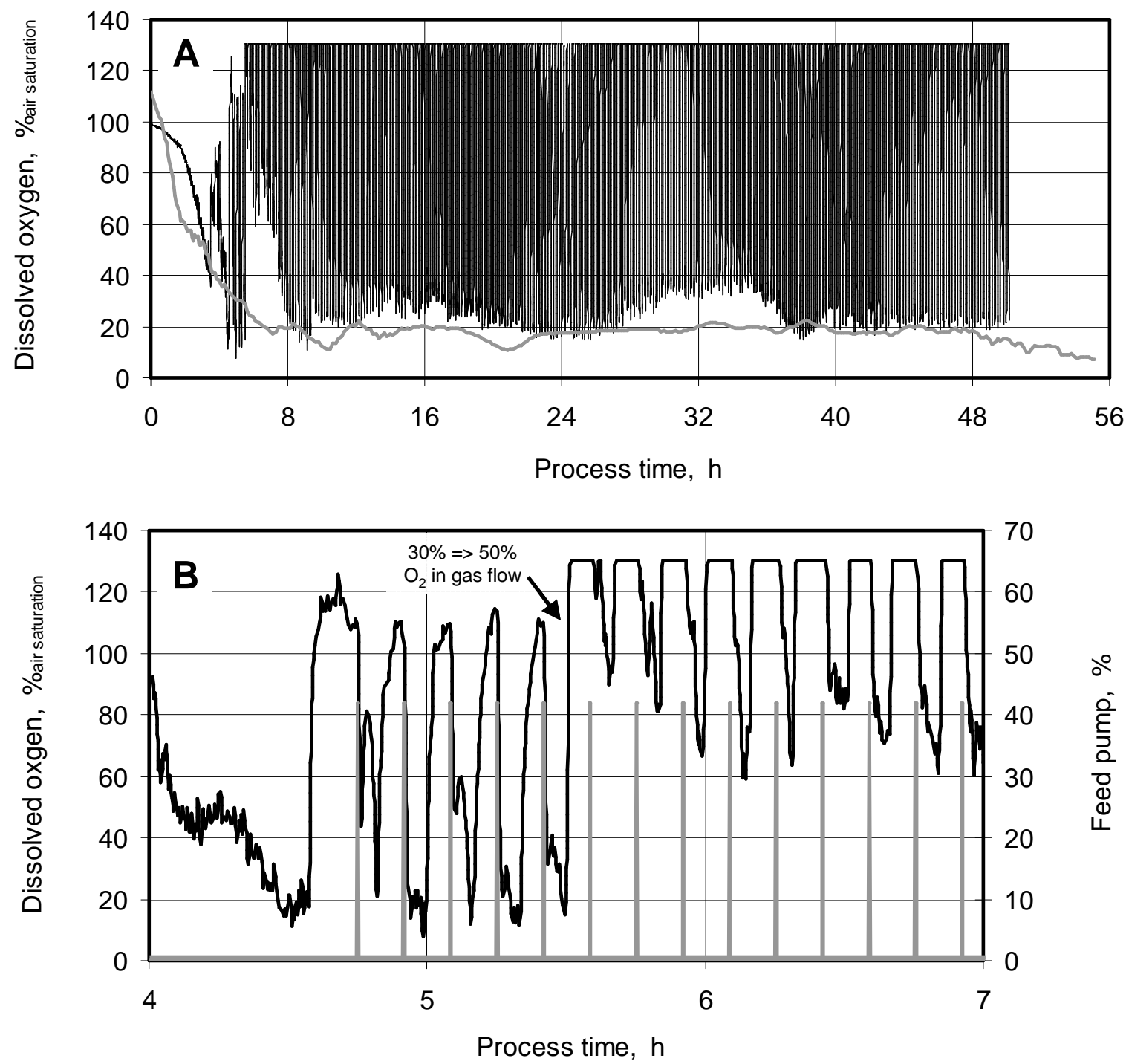

Figure 4-19: A: Dissolved oxygen $(D O)$ profile for a B2 process at laboratory scale (black line), operated with intermittent feed and base addition every 10 min according to the protocol envisioned for the milliliter setup compared to the continuously fed reference process. Dissolved oxygen values $>130 \%$ could not be measured by the used DO probe. B: Focus on the dissolved oxygen (black line) at the end of the batch and beginning of the feeding phase, during which the pulsed feed addition is indicated by the feed pump activity (grey line). At 5.5 hours the oxygen fraction in the inlet gas flow was raised from $30 \%$ to $50 \%$. 
The dissolved oxygen profile of this process is presented in Figure 4-19 A, reflecting the massive oscillations of the intermittent system. The zoom into the course of the dissolved oxygen (Figure 4-19 A) revealed little dissolved oxygen peaks within some of the 10 min intervals after the initial steep drop of the dissolved oxygen before the signal climbed back up. The detection of this phenomenon, which was either caused physically by lingering droplets of feed solution and thereby a second provision of part of the substrate after a short break or biologically by metabolic shifts, was regarded reliable due to a response time of the dissolved oxygen probe in the range of 10-15 s (Puskeiler, 2004).

The steep rise of the dissolved oxygen signal generally indicates the complete depletion of all carbon sources. The periods between the glucose addition and this increase of the signal, which were in the range of three to five minutes when the small peaks were neglected, allowed an estimation of the time for glucose consumption during the respective interval. Together with the amount of glucose added with each pulse, the glucose uptake rates for individual intervals could be assessed. Based on these values, the specific glucose uptake rates were determined for two intermittent B2 processes at different times during the cultivation, using the dry cell mass present in the reactor at the respective times (Figure 4-20).

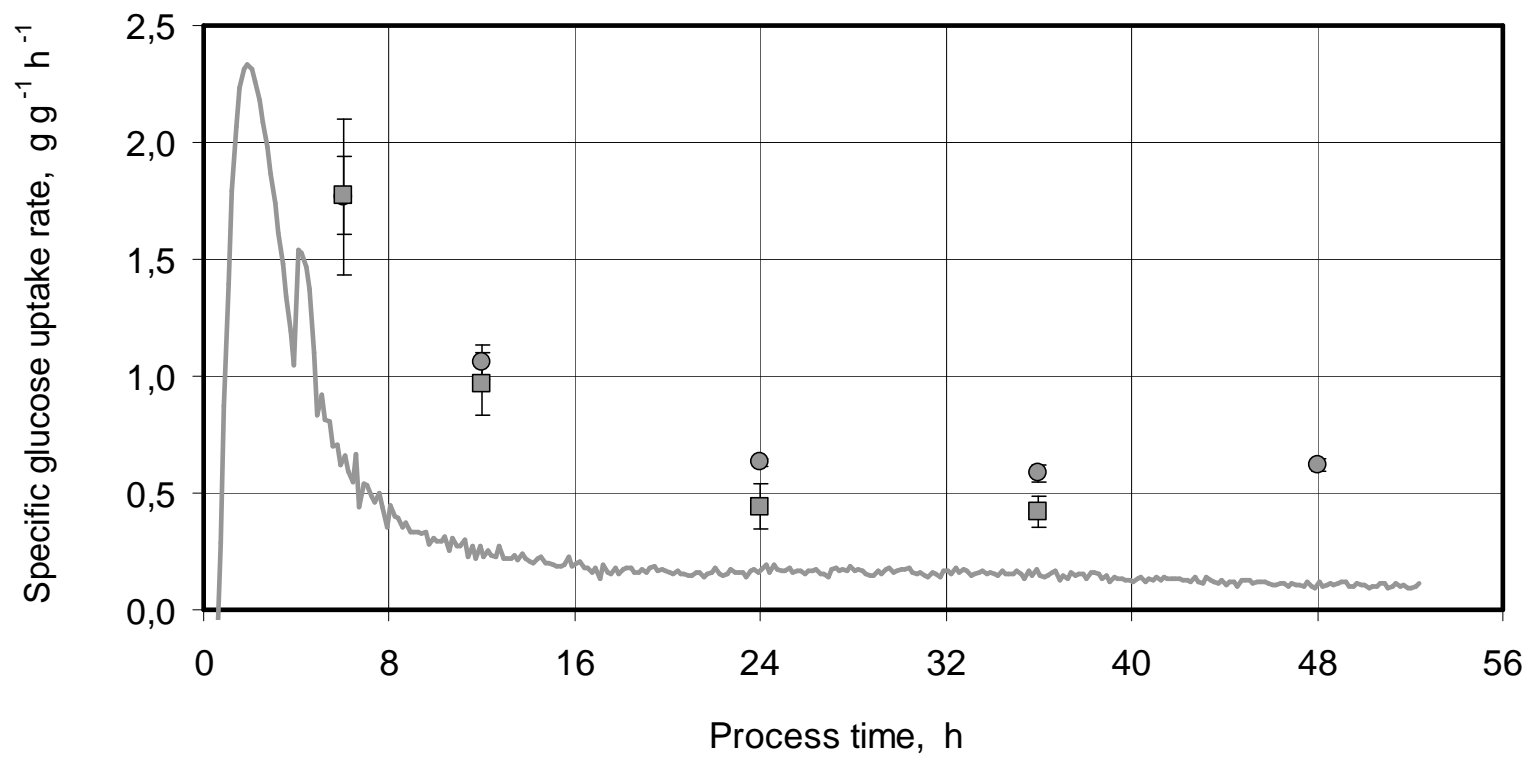

Figure 4-20: Specific glucose uptake rates during two intermittent B2 processes, operated either with feeding intervals of $5 \mathrm{~min}$ and continuous base addition (grey squares) or with $10 \mathrm{~min}$ intervals for feed and base (grey circles). The uptake rates were estimated based on the times between a glucose addition and the following steep increase of the dissolved oxygen. Error bars represent the standard deviation for three consecutive intervals around the specified process time. The values are compared with a continuously fed reference process, for which the data are based on a regression using the feed balance values, the reactor volume and the biomass concentration (grey line). 
The specific glucose uptake rates, determined according to this method, were almost the same for the intermittent processes and clearly higher than during the continuously fed reference with $1.77 \pm 0.3 \mathrm{~g} \mathrm{~g}^{-1} \mathrm{~h}^{-1}$ after 6 hours and $0.62 \mathrm{~g} \mathrm{~g}^{-1} \mathrm{~h}^{-1}$ in the end, compared to $0.66 \mathrm{~g} \mathrm{~g}^{-1} \mathrm{~h}^{-1}$ and $0.10 \mathrm{~g} \mathrm{~g}^{-1} \mathrm{~h}^{-1}$, respectively. This corresponds to a decrease of the rates during the feeding phase of $65 \%$ for the intermittent cultivations and $85 \%$ for the continuously fed reference.

The $\mathrm{pH}$ during these cultivations was measured online. The online values were not employed for the intermittent $\mathrm{pH}$ control, which was restricted to single values every $30 \mathrm{~min}$. However, they could be used for $\mathrm{pH}$ monitoring during the cultivations (Figure 4-21), which revealed oscillations of generally 0.3 and in some cases up to $0.5 \mathrm{pH}$ units within a 10 min feeding interval (Figure 4-21 B).

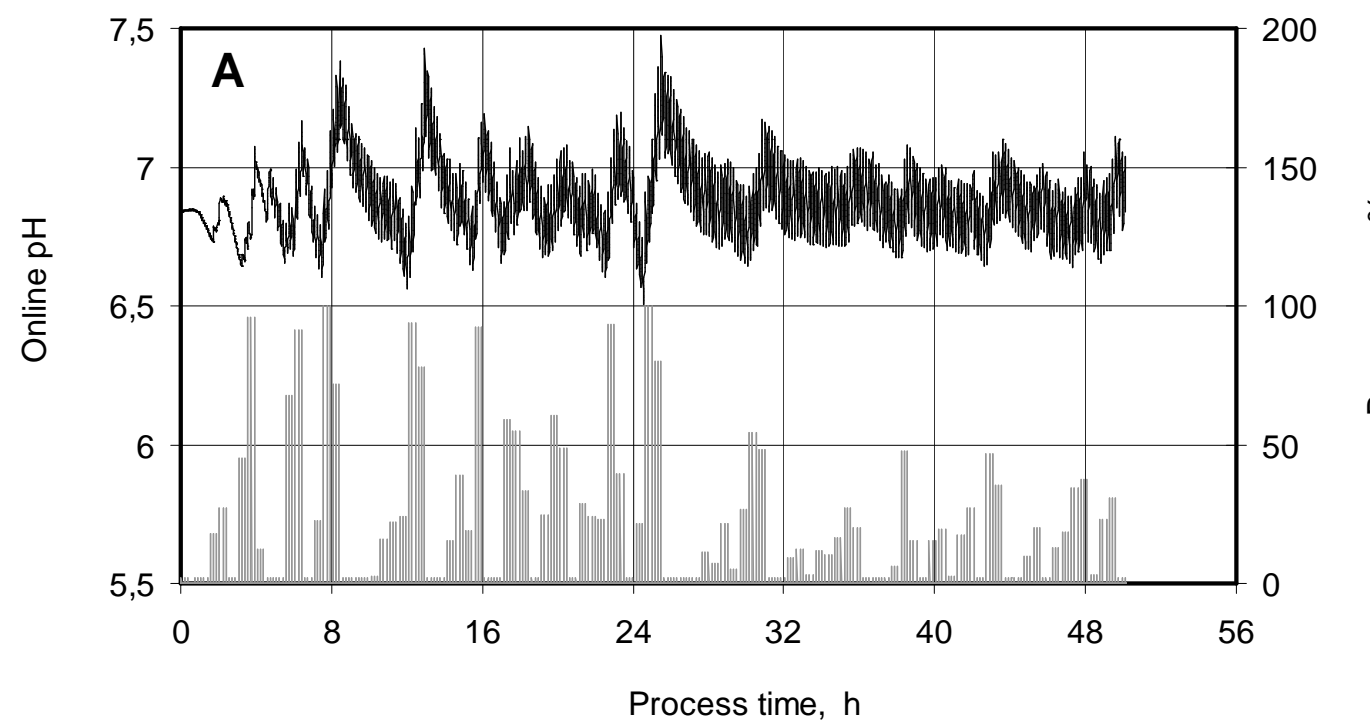

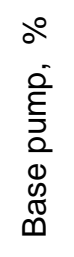

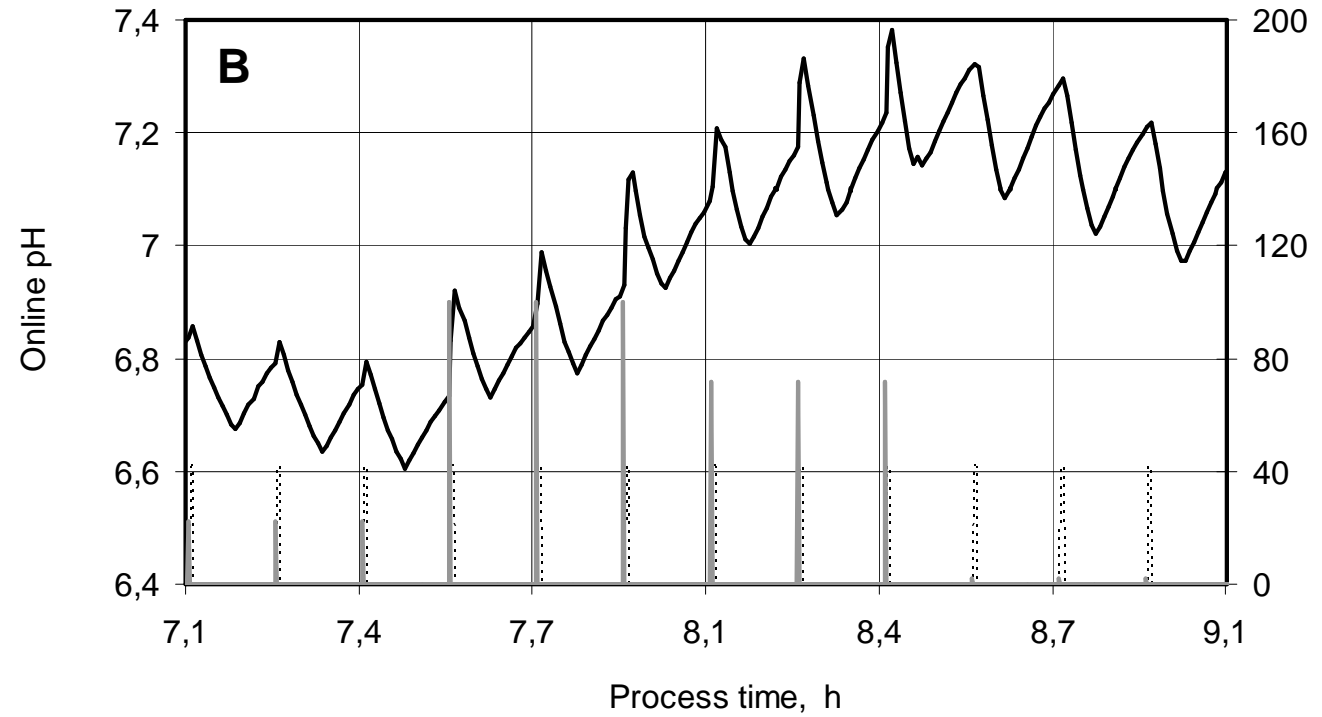

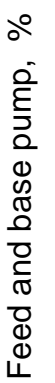

Figure 4-21: A: Online $p H$, feed pump and base pump activities (solid black line, dotted black line and solid grey line, respectively) during an intermittently operated B2 process at laboratory scale, operated with 10 min feeding and base addition intervals. The $\mathrm{pH}$ during this cultivation was controlled based on $\mathrm{pH}$ values used only once every $30 \mathrm{~min}$. B: Focus on two hours during the feeding phase of the cultivation. 
After each addition of base, the $\mathrm{pH}$ rose to a degree that depended on the pump activity, which was equivalent to the inserted base volume, and steeply decreased after the subsequent feed addition. These short-time fluctuations overlaid the deviation of the average $\mathrm{pH}$ from the setpoint of $\mathrm{pH}=6.8$ over more extended periods of time. Overall the resulting $\mathrm{pH}$ range was 6.5 to 7.5 (Figure 4-21 A).

The off-gas analysis enabled the assessment of the carbon dioxide $\left(\mathrm{CO}_{2}\right)$ concentration in the exhaust gas flow, which is presented exemplary for one hour during an intermittent B2 process with a 10 min feeding and base addition interval in Figure 4-22. The $\mathrm{CO}_{2}$ evolution rates (CER) during this cultivation fluctuated between 0.14 and $0.24 \mathrm{~mol} \mathrm{~L}^{-1} \mathrm{~h}^{-1}$ during the time between 18 hours and 32 hours and decreased to values between 0.10 and $0.22 \mathrm{~mol} \mathrm{~L}^{-1} \mathrm{~h}^{-1}$ until the end of the process (data not shown). The local maxima of the $\mathrm{CO}_{2}$ concentration were delayed by up to one minute compared to the feed pulses and to the local minima of the oxygen concentration. The latter one was clearly above the reliable measuring range of the instrument (0-25\% oxygen) for most part of a feeding interval due to the oxygen enrichment of the gas flow.

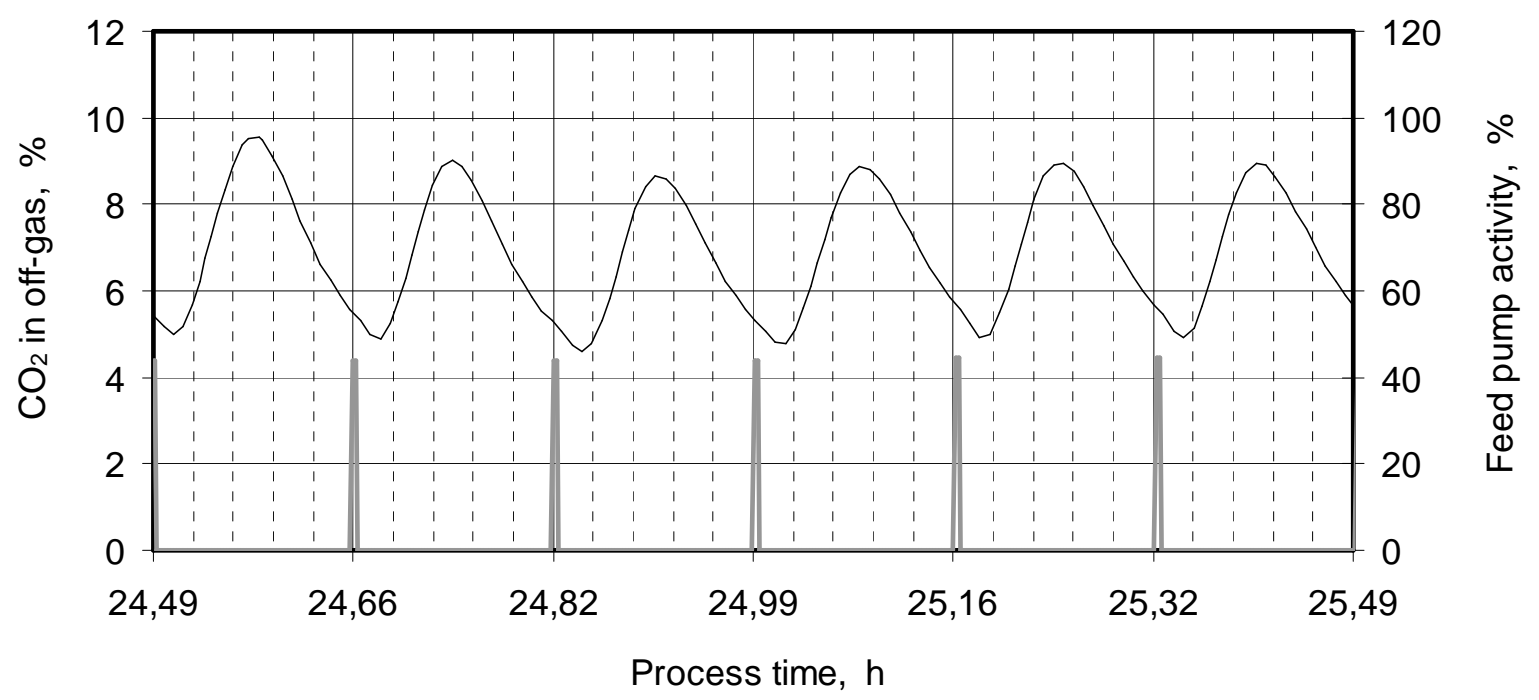

Figure 4-22: Carbon dioxide $\left(\mathrm{CO}_{2}\right)$ concentration (black line) in the off-gas flow during one hour of an intermittently fed B2 process at laboratory scale. Feed and base were added every $10 \mathrm{~min}$. The feed pump activity is indicated by the bold grey line.

\section{Discussion}

The use of elevated oxygen tensions provided challenges on the assessment of both oxygen concentrations in the exhaust gas and dissolved oxygen in the medium, because the values were often out of the reliable measuring range of the instruments. Therefore, the calculation of oxygen uptake rates and respiratory coefficients for these experiments was not possible for the most part. However, the major dissolved oxygen oscillations (Figure 4-19) caused by the pulsed feed- 
ing mode clearly indicated temporarily significantly increased oxygen uptake rates. Therefore, the provision with oxygen in the gas flow for the aeration during intermittently fed B2 processes had to be higher than during continuously fed cultivations under non-pressurized conditions. Only during B2 processes with significantly increased oxygen supply was the reproduction of the final riboflavin concentrations possible. The productivity under the described oscillating conditions reached the reference values when a minimum of $50 \%$ oxygen in the gas flow was supplied during the feeding phase (Figure 4-18).

The rise of the $\mathrm{CO}_{2}$ concentration in the off-gas was delayed by up to one minute, when compared to the pulsed feed provision. A delay of the signal due to the length of the tubing between the reactor outlet and the gas analyzer may be disregarded as a cause for the observed lag period for two reasons. Firstly, the gas reached the analyzer after less than five seconds, based on the superficial gas velocity in the tubing, and secondly the oxygen fluctuations occurred without a delay (data not shown). The more likely cause for the shifted $\mathrm{CO}_{2}$ course is the initial dissolution of the gas after the substrate addition, holding back the $\mathrm{CO}_{2}$ until the gas got stripped out of the medium into the exhaust gas with the observed postponement. The dissolution of $\mathrm{CO}_{2}$ may also provide an explanation for the decrease of $\mathrm{pH}$ after each addition of substrate (Figure 4-21). When $\mathrm{CO}_{2}$ dissolves in the medium, it forms carbonic acid which dissociates and thus lowers the $\mathrm{pH}$ temporarily. Zeng (1995) presented an equation to account for the influence of $\mathrm{CO}_{2}$ absorption and dissociation on the measurement of the $\mathrm{CO}_{2}$ evolution rate, incorporating the dependence on $\mathrm{pH}$ and gas flow rates. However, this equation was developed for continuous cultures and may not easily be applied to pulsed fedbatch experiments.

Another possible explanation for the decrease and rise of the $\mathrm{pH}$ is the generation and subsequent consumption of acetic by-products due to overflow metabolism within one feeding interval. This hypothesis is supported by the dissolved oxygen signal (Figure 4-19). The small peaks after the abrupt drop in dissolved oxygen, following each glucose addition, indicate that after the consumption of the glucose another carbon source is available for utilization. Enfors et al. (2001) reported a repeated production and re-assimilation of acetate from overflow metabolism in $E$. coli within minutes during experiments, during which the cells were exposed to constantly alternating high glucose and low oxygen conditions or glucose limitation and sufficient oxygen. They suggested this phenomenon as a possible cause for reduced biomass concentrations in large scale reactors, in which imperfectly mixed zones of nutrients occur. Fuhrer et al. (2005) suggested that overflow metabolism was not a typical microbial trait, but rather an adaptation to industrial or laboratory conditions, based on the quantification of glucose metabolism in seven 
bacterial species including Bacillus subtilis. In the strain of $B$. subtilis used during this work, overflow metabolism occurs, if glucose is provided clearly above a critical substrate concentration, which is the reason why a glucose-limited fed-batch operation is employed for the original B2 process protocol. Dauner et al. (2001b) reported acetate formation as a primary result of overflow metabolism under conditions of glucose excess during chemostat cultivations with $B$. subtilis, accompanied by the generation of acetoin and diacyl compounds. In contrast to this, the major side product during fed-batch B2 processes with continuous feeding at DSM NP is the $\mathrm{pH}$-inactive acetoin rather than acetate (Hohmann, 2004). This implies that the theory of the generation and subsequent consumption of acetic byproducts is less likely as an explanation for the $\mathrm{pH}$ shifts.

During the intermittent B2 processes, the regularly occurring overfeeding at the beginning of each interval may have been sufficient to induce the transcription of the enzymes expressed for overflow metabolism. The glucose uptake rates during these cultivations were above or right around $0,63 \mathrm{~g} \mathrm{~g}^{-1} \mathrm{~h}^{-1}$ at the beginning of every feeding interval (Figure 4-20) before the substrate was completely consumed after a few minutes. This was the value of the specific glucose uptake rate, above which a transcription of the overflow enzymes still took place during the continuously fed process (Hohmann, 2004). A transcriptome analysis of an intermittently fed $B$. subtilis culture could possibly elucidate, if the enzymes for overflow metabolism would be transcribed throughout the complete course of a cultivation operated with this feeding mode.

Time-resolved analytical data for by-products were not available to support the discussed assumptions. However, a lasting accumulation of by-products did certainly not occur since both sides of the carbon balances were equivalent at the end of all the presented processes (data not shown). Only during a small number of cultivations did temporary gaps in the carbon balance suggest the existence of undetected compounds at those times (data not shown).

The deviations of the mean $\mathrm{pH}$ values from the setpoint could be attributed to the rather simple nature of the $p H$ control algorithm ${ }^{1}$. For a more precise $p H$ adjustment a more refined controlling approach would be necessary. However, the results demonstrate that all the observed fluctuations were tolerable for an appropriate performance of $B$. subtilis with respect to its riboflavin production. Nevertheless, the order of the addition of feed and base during B2 processes at milliliter scale should be swapped to keep the oscillations within one feeding interval in a more narrow range.

\footnotetext{
${ }^{1}$ The control algorithm for the intermittent $p H$ control is described in Appendix 7.2.8.
} 
The temporarily occurring oxygen or glucose limitations and shifts in $\mathrm{pH}$ may have triggered certain stress responses, like the synthesis of so called $\sigma^{\mathrm{B}}$-dependent general stress proteins in B. subtilis (Kovács et al., 1998). Schweder et al. (1999) examined the mRNA levels of four different stress proteins in $E$. coli in response to defined high glucose and low oxygen areas. They reported elevated mRNA levels for the oxygen limitation genes within seconds after the cells passing through the designated zone. In their experiments a mean residence time of $10 \mathrm{~min}$ in the glucose-limited and oxygen-sufficient area was enough to relax the stress response by means of a reduction of the respective mRNA level. This is in agreement with the short half life of mRNA, which is in the range of only a few minutes. This study showed that the bacteria continuously tried to adapt to an environment that only lasted for a few seconds. In addition to these findings, Enfors et al. (2001) reported that the described fluctuations could be beneficial to the viability of $E$. coli as analyzed by flow cytometry.

One can assume similarly fluctuating stress responses in $B$. subtilis to the shifts between limitation and excess of glucose and oxygen during the intermittently fed B2 cultivations, indicating that the overall physiology of the cells was altered compared to processes with continuously available nutrients. This may explain why the final dry cell mass, under the conditions described in this work, was significantly lower than during reference processes, which were operated at lower oxygen tension, yielding approximately $32 \mathrm{~g} \mathrm{~L}^{-1}$ compared to $40-45 \mathrm{~g} \mathrm{~L}^{-1}$ (Figure 4-18). However, the pulsed feeding mode alone cannot serve as the reason for the lower dry cell mass. This phenomenon was more likely to be caused by the significantly elevated dissolved oxygen tensions due to the necessary oxygen addition, because it was also observed for the continuously fed cultivation, during which $50 \%$ oxygen was present in the gas flow at ambient pressure. This resulted in dissolved oxygen values above $160 \%$ (data not shown) and clearly reduced dry cell mass during the second half of the process. Similarly low cell concentrations were also observed during the intermittent experiment with $35 \%$ oxygen in the gas flow (Figure 4-18 A), during which the excess pressure of 350-450 mbar triggered respectively higher dissolved oxygen.

It was not possible to distinguish between living and dead cells in the total dry cell mass. But as the overall riboflavin productivity was not necessarily lower during the cultivations with decreased final cell mass, it is safe to assume that the metabolically active fraction of cells remained unaffected by the higher oxygen tension and only the inactive or dead cells could not withstand the oxidative stress and were object to cell lysis, yielding a "fitter" remaining population. Another support of this hypothesis is provided by the fact that the cell concentration did not seem to be influenced by high dissolved oxygen values during the first few hours 
of the feeding phase when the fraction of non-viable or inactive cells of the total cell population would be very low anyway.

All of these factors lead to the conclusion that, whatever the reason for the observed lower dry cell mass concentrations is, this phenomenon causes an increased apparent specific productivity of the cell population and elevated specific glucose uptake rates (Figure 4-20) compared to the continuously fed B2 processes. The fact that the specific glucose uptake rate in these experiments decreased with process time could not be solely attributed to the increasing cell concentrations (as is the case during the continuously fed process), because theoretically the cells could have exhausted the glucose faster, since they received the complete amount of feed for one interval unrestricted within the first five to 20 seconds of a feeding cycle. The declining uptake rates would indicate a reduction of the specific metabolic activity of the culture. If, on the other hand, the small peaks before the steep rise of the dissolved oxygen signal were considered as an indication for by-product metabolism after the initial glucose consumption, this would not have to be the case. In this case, the uptake rates for glucose could still be high and only appear to be lower due to the use of false dissolved oxygen signals for their estimation.

However, it is most likely that a decreased specific metabolic activity with respect to the glucose uptake rate was the case for both the continuously and the intermittently fed cultivations toward the end of a B2 process. This was confirmed for continuously fed processes by raising the feeding rate at the end of some processes. During two of these experiments twofold or eightfold enhancement of the feed pump speed did not result in twofold or eightfold increase of the specific glucose uptake rate, but resulted in only $50 \%$ or $225 \%$ augmentation, yielding 0.18 or $0.27 \mathrm{~g} \mathrm{~g}^{-1} \mathrm{~h}^{-1}$, respectively, compared to $0.12 \mathrm{~g} \mathrm{~g}^{-1} \mathrm{~h}^{-1}$ before. This could be calculated based on the rate of glucose accumulation ${ }^{2}$. Thus the final cell population metabolized the provided glucose with only approximately $8-12 \%$ of the maximum specific glucose uptake rate.

It is surprising that the original continuously fed B2 process is operated at feed rates of $41 \mathrm{~g} \mathrm{~h}^{-1}$ throughout the complete feeding phase for processes with an initial volume of $3 \mathrm{~L}$, because this translates to a specific feed rate as low as $0.12 \mathrm{~g} \mathrm{~g}^{-1} \mathrm{~h}^{-1}$ at elevated process times, if the total dry cell mass is considered for the calculation. This would allow glucose uptake just sufficient for the maintenance metabolism of $B$. subtilis, according to the maintenance coefficient on glucose reported for similar riboflavin-producing $B$. subtilis mutant strains in chemo-

\footnotetext{
${ }^{2}$ Refer to Figure 4-29 in section 4.4.3 Monitoring of the glucose concentration.
} 
stat cultures, and compared to $0.08 \mathrm{~g} \mathrm{~g}^{-1} \mathrm{~h}^{-1}$ for the respective wild-type (Sauer et al., 1996; Zamboni et al., 2003). According to more recent publications by Dauner and Sauer (2001), however, the maintenance coefficient for B. subtilis is even as high as $0.21 \pm 0.04 \mathrm{~g} \mathrm{~g}^{-1} \mathrm{~h}^{-1}$. This would support the hypothesis that only a part of the cell population at elevated process times is metabolically active with respect to glucose uptake, given that the maintenance coefficient represents the minimum specific glucose uptake rate.

\subsubsection{Summary}

The preceding subsections provided the results of B2 processes at laboratory scale after sequential modifications to simulate the operation envisioned for processes at the milliliter scale. This included the stepwise change from continuous to intermittent substrate and base addition every $10 \mathrm{~min}$, the increase of the oxygen transfer rate by enriching the gas flow with oxygen at ambient pressure and constant stirrer speed, and the application of an intermittent $\mathrm{pH}$ control, which was restricted to using $\mathrm{pH}$ values only once every $30 \mathrm{~min}$.
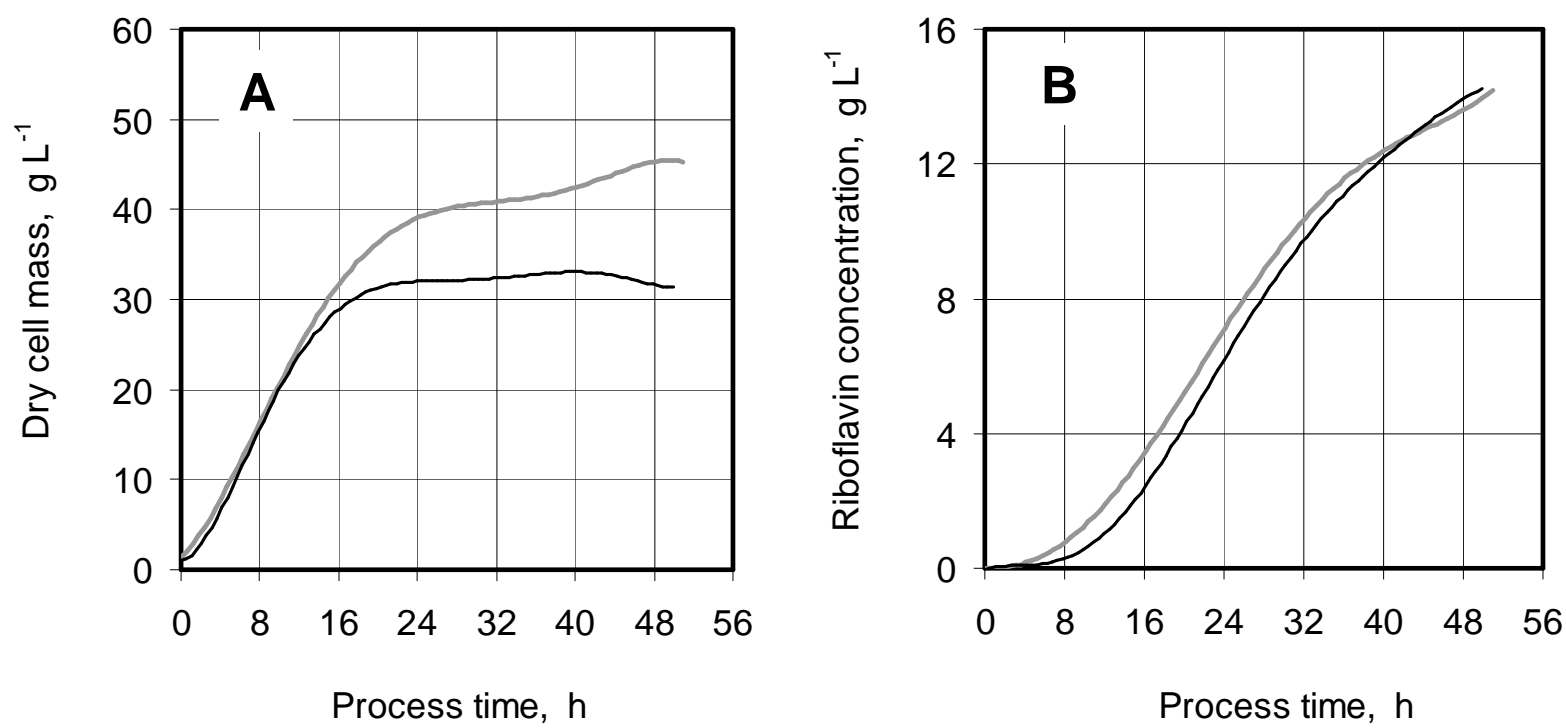

Figure 4-23: Growth (A) and riboflavin production (B) during the reference B2 process, operated with continuous feeding according to the original protocol (grey line), and during an intermittent B2 process at laboratory scale, operated according to the protocol envisioned for the milliliter setup (black line).

In summary, the experiments demonstrated that these modifications caused significant oscillations in dissolved oxygen, exhaust gas concentrations and $\mathrm{pH}$, which were primarily caused by the intermittent substrate addition. There was some evidence for the temporary formation and consumption of by-products within a feeding cycle, but insufficient evidence to prove this hypothesis conclusive. Lasting accumulation of substrate or by-products did not occur. Despite the 
described fluctuations, the riboflavin productivity was not significantly affected except for a delayed onset of the productivity by approximately two to three hours (Figure 4-23 B). The final product concentrations of the reference could only be reached if sufficient oxygen for the temporarily increased oxygen uptake rates was supplied during the feeding phase. Intermittent feeding intervals of 10 min required at least $50 \%$ oxygen in the inlet gas flow. In this case, however, the final dry cell mass was approximately 20-30\% lower than during the reference process (Figure 4-23 A), which was most likely caused by the elevated dissolved oxygen tensions. While the overall riboflavin generation remained unaffected, this phenomenon yielded an apparently increased specific productivity.

Since the reproduction of the riboflavin productivity of $B$. subtilis was the crucial criterion for the adaptation of the B2 process to the milliliter setup as a tool for screening, the observed influences of the operational changes were considered acceptable and the described process conditions were applied for cultivations in the milliliter bioreactors. 


\subsection{Parallel analyses for the automated milliliter process}

The first part of this section introduces the overall concept of the at-line process analysis in the milliliter setup. Subsequent subsections describe the establishment of individual methods for monitoring of growth, substrate consumption and product formation. The section concludes with a summary of the findings.

A laboratory robot and a photometric plate reader for absorbance and fluorescence measurements in microtiter plates were available for sample handling and at-line analyses during the automated B2 processes in the milliliter setup. A separation of cells from the process samples was not intended, although particles would disturb absorbance measurements in a sample due to light scattering. For this reason very high sample dilutions should be applied and fluorimetric measurements should be employed for the quantification of glucose and riboflavin, because this method is characterized by a considerably higher sensitivity compared to the determination of a component via absorbance.

\subsubsection{Principle of the "all-in-one" analysis}

The time that was available for robotic actions between two intervals of feed and base addition was limited to a few minutes and had to be used as efficiently as possible for the at-line analysis. Therefore, a dilution scheme was developed that would allow all the necessary analyses to be carried out from only one sample per reactor ("all-in-one" analysis). This scheme had to provide the appropriate dilution for each of the desired analyses to meet the required measuring range.

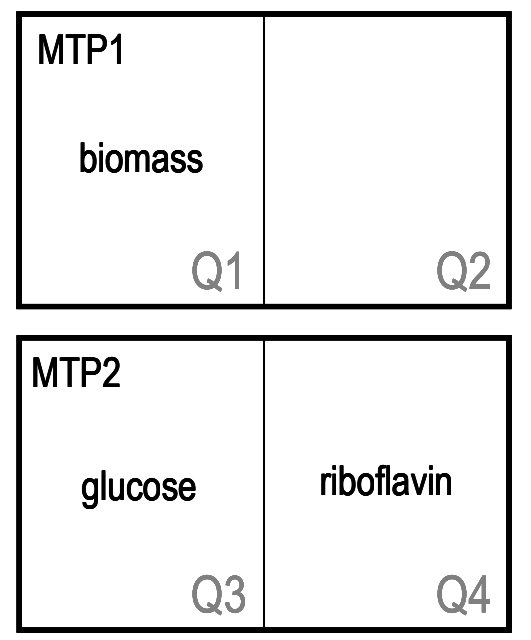
Q1: $\mathrm{NaOH}+$ sample + citric acid $\quad$ dilution $=1: 18$ (dry cell mass via optical density)
Q2: buffer + Q1 $\quad$ dilution $=1: 432$
Q3: buffer + Q2 dilution $=1: 5400$
(plus reactive enzymatic dye solution in the MTP reader right before the glucose measurement) $=>$ dilution $=1: 10800$
Q4: buffer + Q3 dilution $=1: 54000$
(riboflavin content via intrinsic fluorescence)

Figure 4-24: Schematic display of the two microtiter plates (MTPs) used for the "all-in-one" analysis and the applied dilution steps to measure optical density, glucose and riboflavin concentration. 
The resulting pipetting routine consisted of four dilution steps carried out in two microtiter plates, which were divided into four sectors of 48 wells Q1-Q4, as shown schematically in Figure 4-24. Thereby samples from 48 reactors could be analyzed quasi-simultaneously in parallel. Sample volumes $<20 \mu \mathrm{L}$ and transfer volumes $<10 \mu \mathrm{L}$ were avoided because of the limited accuracy of the laboratory robot for pipetting smaller volumes.

The samples were taken from the vessels and were added to $1 \mathrm{M}$ sodium hydroxide $(\mathrm{NaOH})$ solution in $\mathrm{Q} 1$ for the dissolution of riboflavin crystals. Next, $0.3 \mathrm{M}$ citric acid was added for neutralization in order to avoid degradation of riboflavin or shrinking of cells until the actual measurement, yielding a dilution in Q1 of 1:18. $10 \mu \mathrm{L}$ from $\mathrm{Q} 1$ were transferred into Q2, from where the same amount was taken into Q3. The first plate was then ready for the photometric measurement. After the final dilution step from Q3 into Q4, the second plate was also ready for analysis. $100 \mu \mathrm{L}$ of a reactive enzymatic dye solution was added to the 1:5400 diluted samples in Q3 for the assessment of the glucose concentration. The final dilution of 1:54000 in Q4 allowed the fluorimetric quantification of riboflavin.

The dilution steps of the developed scheme did not only have to provide the appropriate concentration of each analytical quantity to be determined in the different sectors of the microtiter plates, but also the $\mathrm{pH}$ had to be suitable for the glucose detection. This was guaranteed by diluting small sample volumes in large volumes of buffer with $\mathrm{pH}=7.4$ and by the neutralizing step with citric acid in $\mathrm{Q} 1$.

\subsubsection{Estimation of the dry cell mass}

The dry cell mass concentration $c_{X}$ of the cultivation samples was determined by measuring the optical density $O D$ of $230 \mu \mathrm{L}$ diluted cell suspension in $1 \mathrm{M} \mathrm{NaOH}$ in Q1 at $650 \mathrm{~nm}$ without the addition of acid. This step was added later during the development. A correlation of $c_{X}$ to $O D_{650}$ was established using the gravimetrically determined dry cell mass data from several B2 processes at laboratory scale, of which the $O D_{650}$ had been determined in the plate reader after dilution with the laboratory robot. The results are depicted in Figure 4-25.

It was challenging to find a correlation for the complete range of dry cell mass, due to the fact that the $O D$ values were more scattered at high biomass concentrations. This was also the case when the optical density of respectively diluted samples was measured in $1 \mathrm{~mL}$ cuvettes (data not shown). However, a polynomial regression of fourth degree (Equation 4-1), displayed as a line in Figure 4-25, did provide an appropriate mapping of the $O D$ values with $\mathrm{R}^{2}=0.98$. 


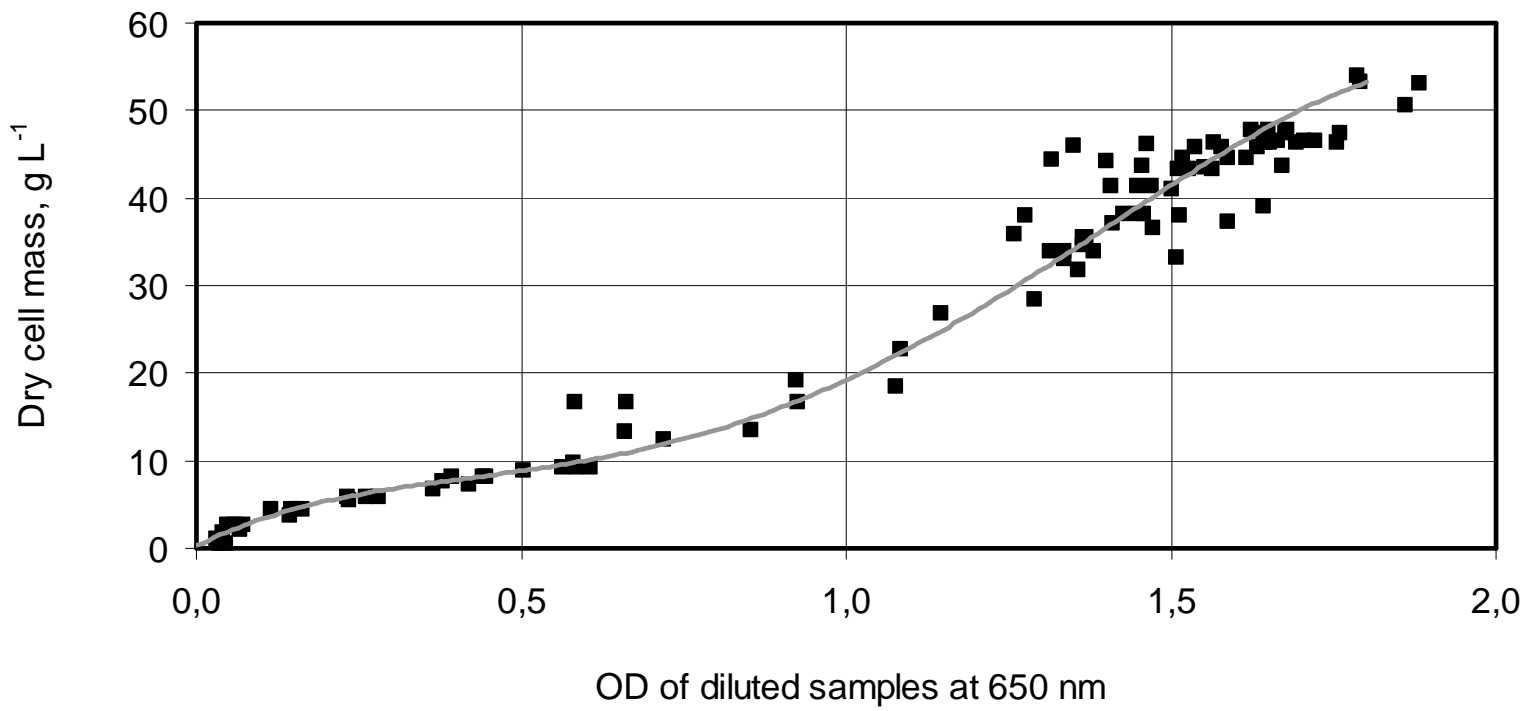

Figure 4-25: Correlation between the gravimetrically determined dry cell mass $c_{X}$ of samples from three independent B2 processes at laboratory scale and their optical density at $650 \mathrm{~nm}$ measured in $230 \mu \mathrm{L}$ diluted samples in microtiter plates in the photometric plate reader (dilution 1:12 with the laboratory robot, sample volume $=20 \mu \mathrm{L}$ ), represented as black symbols. The grey line represents the correlation which was calculated by polynomial regression $\left(R^{2}=0.98\right)$.

Equation 4-1:

$$
C_{X}=-22.55 \cdot O D_{650}{ }^{4}+81.22 \cdot O D_{650}{ }^{3}-79.09 \cdot O D_{650}{ }^{2}+39.41 \cdot O D_{650}+0.16
$$

With this correlation, the dry cell mass concentration could be estimated for $c_{X}$ $\approx 2.5-55 \mathrm{~g} \mathrm{~L}^{-1}$ after the first dilution step in Q1 by measuring the optical density at $650 \mathrm{~nm}$ either in $230 \mu \mathrm{L} 1 \mathrm{M} \mathrm{NaOH}$ or in $350 \mu \mathrm{L}$ of $\mathrm{NaOH}$ plus citric acid.

\section{Discussion}

The light scattering properties of the cells obviously changed during a cultivation, which could be concluded from higher correlation factors between the dry cell mass and the $O D$ during the initial phase of the laboratory scale experiments, compared to reduced coefficients after elevated process time. This is consistent with observations in shake flask cultures, for which the correlation factor was also relatively high (data not shown). This phenomenon could be addressed by using a polynomial regression, which accounted for the correlation during the complete process time.

Originally the pipetting for the "all-in-one" analysis had been established without the addition of acid to Q1. The optical density of the samples in $\mathrm{NaOH}$ decreased over time during initial tests, but became stable after approximately 20 minutes (data not shown). During later experiments this could not be consistently repro- 
duced. In addition, the extended time between sampling into $\mathrm{NaOH}$ and the actual measurements during the automated B2 processes bore the risk of riboflavin degradation due to the highly basic $\mathrm{pH}$. Therefore, the addition of citric acid was introduced into the scheme, resulting in significantly more stable values for the optical density, even after elevated incubation time (data not shown). The same polynomial correlation was still applicable. The increase in volume and therefore a longer light path through the sample, together with the lower concentration of $\mathrm{NaOH}$, most likely made up for the decrease of the $O D$ due to the sample dilution.

\subsubsection{Monitoring of the glucose concentration}

A method for the fast assessment of glucose in cell-containing cultivation samples was developed, based on a commercially available glucose detection kit (Amplex ${ }^{\circledR}$ Red Glucose/Glucose Oxidase Assay Kit, Molecular Probes, Eugene, OR, USA).

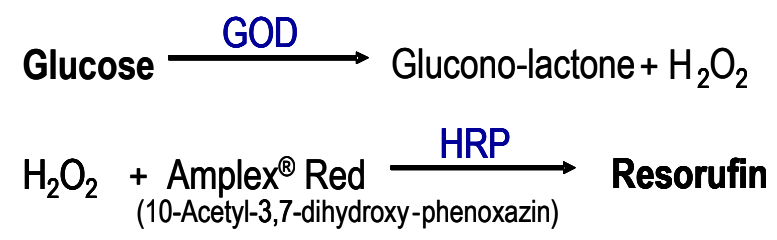

Figure 4-26: Equimolar enzymatic reactions responsible for the generation of fluorescence during the commercially available Amplex ${ }^{\circledR}$ Red Glucose Assay. $\mathrm{H}_{2} \mathrm{O}_{2}$, hydrogen peroxide; HRP, horseradish peroxidase; GOD, glucose oxidase.

The assay is based on the enzymatic conversion of the present glucose to glucono-lactone and hydrogen peroxide in the presence of glucose oxidase. Hydrogen peroxide reacts further with a non-fluorescent component 10-acetyl-3,7-dihydroxy-phenoxazine (Amplex ${ }^{\circledR}$ Red), added together with the enzymatic solution, to highly fluorescent resorufin, catalyzed by horseradish peroxidase. These reactions are displayed in Figure 4-26. Both of them are equimolar, thus allowing the correlation of the generated fluorescence to the original glucose concentration.

The fluorescence values, generated 30 minutes after the addition of the reactive solution, could be used for the quantification of glucose, as suggested by the commercial assay description. This was a relatively long time within the scope for the automated analyses in the milliliter setup and the fact that the conversion was not necessarily completed after 30 minutes made an accurate timing of the measurement necessary.

In Figure 4-27, the fluorescence profiles measured after the addition of the reactive dye solution to glucose standards between $0-20 \mu \mathrm{M}$ glucose in a microtiter plate are presented. The profiles for the different concentrations could be clearly 
distinguished by their initial slope. Therefore, the initial increase of fluorescence was correlated to the glucose concentration instead of using the fluorescence values at 30 minutes. The resulting calibration curves for different glucose oxidase concentrations in the reactive solution are depicted in Figure 4-28.

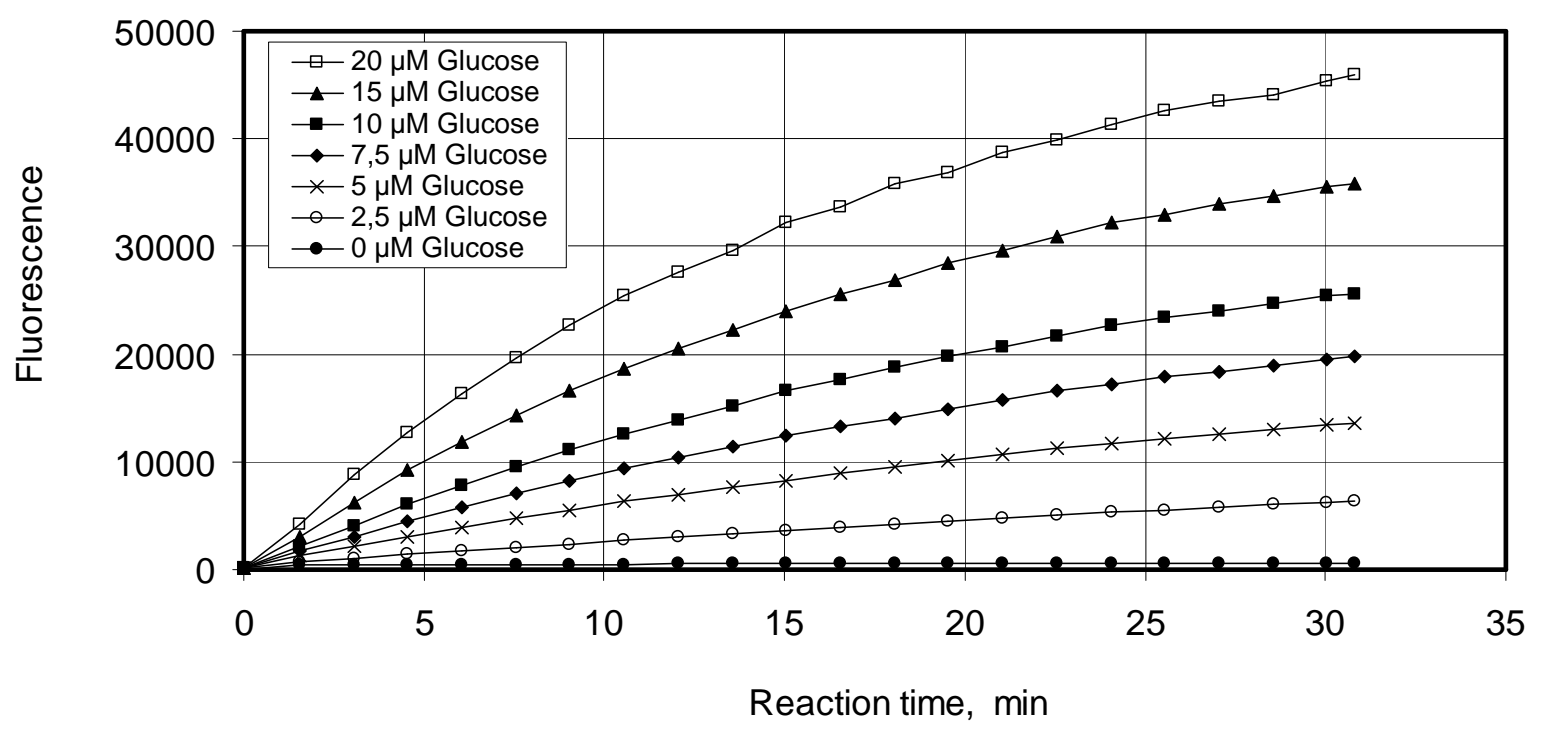

Figure 4-27: Fluorescence measured in arbitrary units every 90 seconds after the addition of the reactive dye solution of the glucose detection kit in a microtiter plate with standards of different glucose concentrations, as indicated by the legend.

The calibration curves in Figure 4-28 revealed a strong dependence of the slope on the glucose oxidase concentration in the reactive solution. Changes of the peroxidase concentration did not have a significant influence (data not shown).

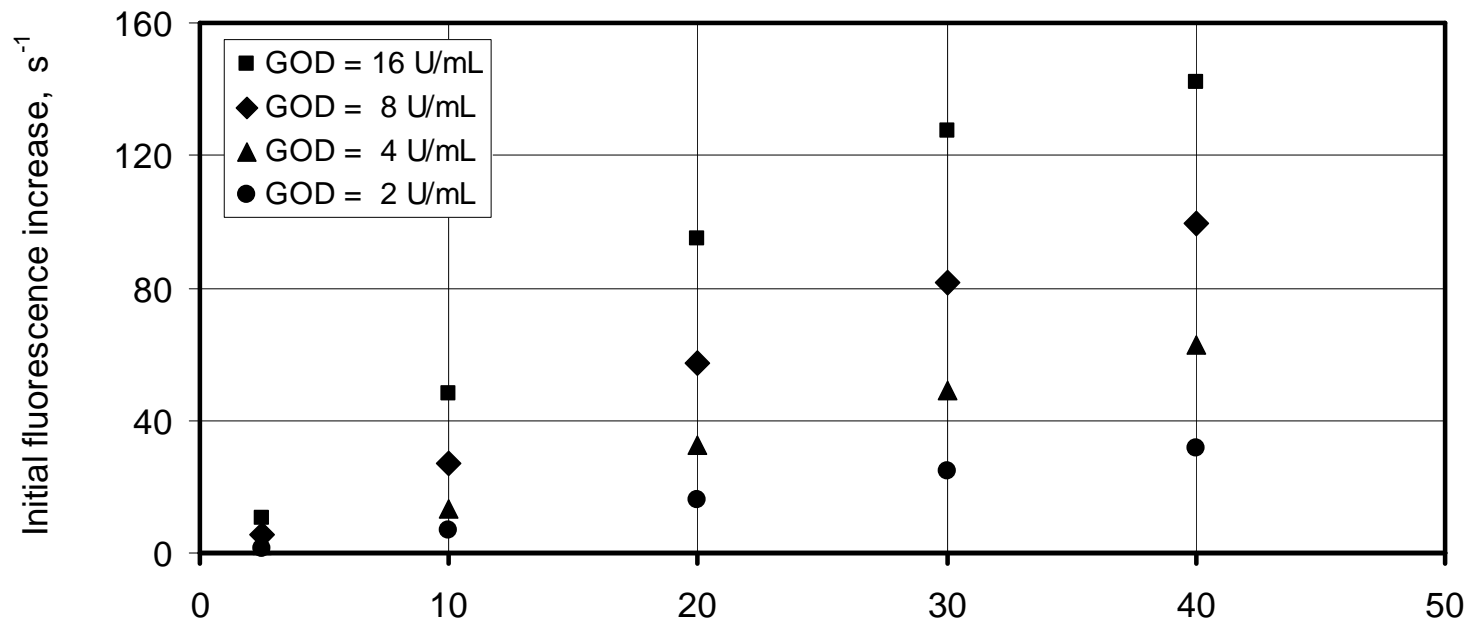

Glucose concentration, $\mu \mathrm{M}$

Figure 4-28: Calibration curves for glucose detection based on the initial slope of the increase of fluorescence after the addition of the enzymatic dye solution, which contained different amounts of glucose oxidase (GOD), indicated in the legend as different enzymatic activities in units per milliliter $(\mathrm{U} / \mathrm{mL})$. 
A glucose oxidase concentration equivalent to an enzyme activity of $8 \mathrm{U} \mathrm{mL}^{-1}$ in the reactive dye solution was chosen for the application of the assay during B2 processes. The measuring range for glucose concentrations in diluted process samples was 2-15 $\mu \mathrm{M}$, when a linear regression was used. This corresponded to 4-29 $\mathrm{g} \mathrm{L}^{-1}$ glucose in the original sample, when a final dilution of 1:10800 was applied. A larger range for higher concentrations could be assessed with non-linear correlations. Lower glucose concentrations could also be detected, but were afflicted with considerable variations.

The spectral range, in which riboflavin is excited to fluoresce, is different compared to that of the fluorescent dye resorufin, which was responsible for the glucose quantification. Experiments, in which riboflavin and glucose standard solutions of different concentrations were mixed, confirmed that riboflavin did not interfere with the glucose quantification (data not shown). However, there was a disturbance of the glucose measurement in culture samples from B2 processes, especially at elevated process time, which could not be assigned to the presence of either cells or riboflavin (data not shown). The general impact of the biomass on the assessment of the glucose concentration in diluted culture samples could not be clarified completely. For details regarding these investigations and for the general establishment of the described method refer to Geipel (2002).

\section{Discussion}

The change of the fluorimetric assay, from using the fluorescence after a certain time to measuring the initial increase of fluorescence after the addition of the enzymatic dye solution, made faster glucose detection possible. The reaction catalyzed by the glucose oxidase is the rate-limiting step in this assay.

The glucose concentration after the batch phase of B2 processes was expected to be close to zero throughout the rest of the cultivation, because a feed rate for glucose-limited conditions was selected. The intention for glucose analysis during the feeding phase was therefore mainly to discover possible metabolic changes, for example due to improper growth conditions, which would most likely manifest in substrate accumulation. In B2 processes at laboratory scale, during which substrate accumulations were caused on purpose by increasing the feed supply by a factor of eight at the end of the process when the dry cell mass was $>40 \mathrm{~g} \mathrm{~L}^{-1}$, the glucose detection was shown to be qualitatively reliable for values $>1.5 \mathrm{~g} \mathrm{~L}^{-1}$, as displayed exemplary for one process in Figure 4-29. The error bars in this figure reveal large variations for the automated glucose detection, most likely due to the limited pipetting accuracy of the laboratory robot. The limitations of the quantitative assessment were considered of minor importance because the assay pro- 
vided the desired information about qualitative changes in the substrate consumption.

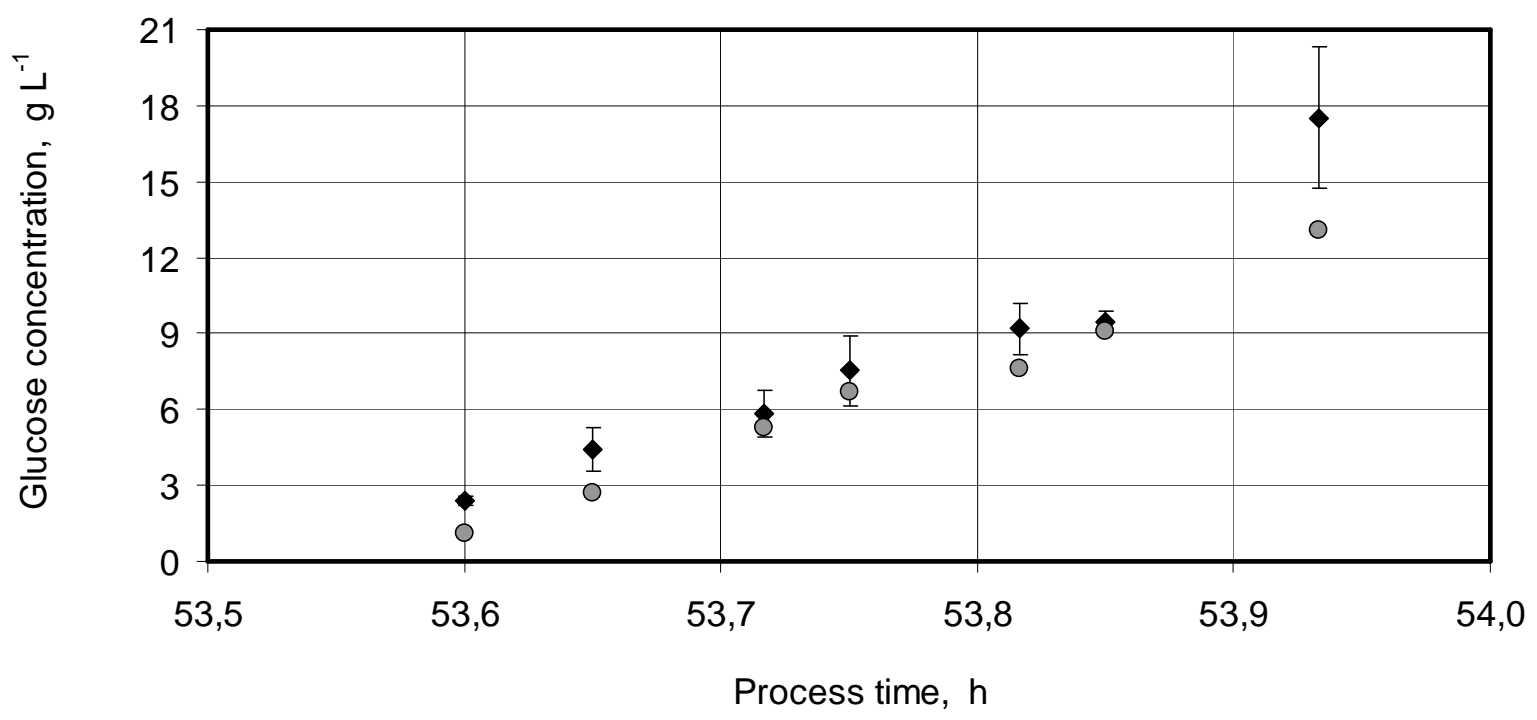

Figure 4-29: Accumulating glucose concentration caused on purpose by increasing the feed supply by a factor of eight at the end of a B2 process at laboratory scale (dry cell mass $>40 \mathrm{~g} \mathrm{~L}^{-1}$ ). Values were measured with the modified enzymatic assay in samples which were diluted with the laboratory robot (black diamonds) or with the offline data of the process, estimated with AccuChek blood sugar test sticks (grey circles). Error bars are based on the standard deviation for triplicates prepared with the robot.

In summary, the modified enzymatic assay provided sufficiently sensitive data for the monitoring of the initial glucose consumption during the batch phase of B2 processes as well as for the detection of glucose accumulations even at high biomass concentration after elevated process time.

\subsubsection{Fluorimetric quantification of the riboflavin concentration}

The riboflavin analysis was based on measuring the intrinsic fluorescence of riboflavin in diluted samples at an excitation wavelength $\lambda_{\text {excitation }}=440 \mathrm{~nm}$ or $485 \mathrm{~nm}$ and emission at $\lambda_{\text {emission }}=520 \mathrm{~nm}$. A linear correlation between the riboflavin concentration and its fluorescence was determined for approximately $0.05-0.5 \mathrm{mg} \mathrm{L}^{-1}$ (data not shown). During the first step of the "all-in-one" analysis, the samples were diluted 1:12 in $1 \mathrm{M} \mathrm{NaOH}$ for complete dissolution of the RF crystals before the addition of neutralizing acid. The total dilution for the fluorimetric quantification was $1: 36000$ or 1:54000 after three further dilution steps, depending on the addition of citric acid, rendering a measuring range of approximately $1.8-18 \mathrm{~g} \mathrm{~L}^{-1}$ or $2.7-27 \mathrm{~g} \mathrm{~L}^{-1}$ riboflavin in the original sample, respectively. 


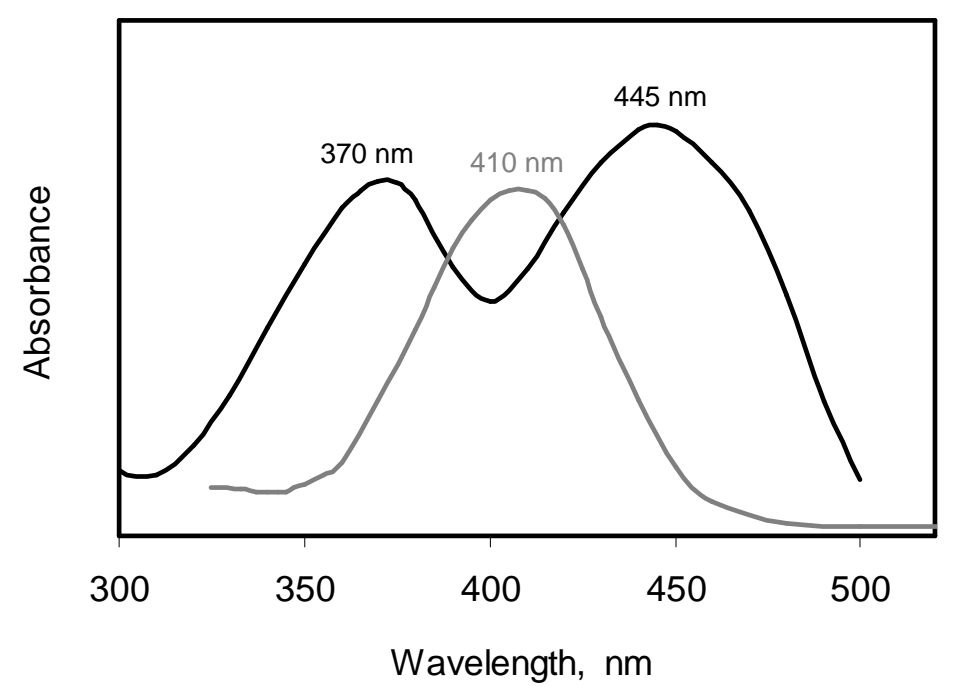

Figure 4-30: Optical spectra for riboflavin (black line) and its biosynthetic precursor di-methyl-ribityllumazine (DMRL; grey line) in independent arbitrary units (DMRL spectrum adapted from Illarionov et al., 2001).

At $\lambda_{\text {excitation }}=440 \mathrm{~nm}$, which is the excitation maximum for riboflavin, by-products or precursors of the riboflavin biosynthesis, for example di-methyl-ribityl-lumazine (DMRL) as the final intermediate before riboflavin, may interfere with the accuracy of the detection by adding to the fluorescent signal.

Therefore, riboflavin and DMRL standard solutions were examined at $\lambda_{\text {excitation }}$ $=485 \mathrm{~nm}$ compared to $440 \mathrm{~nm}$, because DMRL should absorb or be excited only marginally at $\lambda>470 \mathrm{~nm}$ according to its absorbance spectrum (Figure 4-30). The fluorescence of DMRL at $\lambda_{\text {excitation }}=485 \mathrm{~nm}$ disappeared almost completely, whereas it was only approximately $40 \%$ reduced compared to that of riboflavin at $\lambda_{\text {excitation }}=440 \mathrm{~nm}$ (data not shown).

After that the influence of DMRL, added to riboflavin standard solutions, on the fluorimetric quantification of riboflavin was investigated. The employed DMRL concentrations were significantly higher than expected in actual diluted B2 process samples. There was no additive effect of DMRL to the fluorescence when exciting the samples at $485 \mathrm{~nm}$, but instead slightly lower values for all concentrations, including the blank, were observed (Figure 4-31 A).

Next, B2 process samples containing up to $15 \mathrm{~g} \mathrm{~L}^{-1}$ riboflavin were diluted either as usual or with a DMRL standard solution instead of buffer during the last dilution step. In the latter case, the resulting DMRL concentration in the diluted sample corresponded to approximately $10 \mathrm{~g} \mathrm{~L}^{-1}$ in the original sample, which was significantly higher than expected in actual process samples. Also in this case, there was no additive effect of DMRL to the fluorescence using $485 \mathrm{~nm}$ for the excitation of the samples (Figure 4-31 B). 


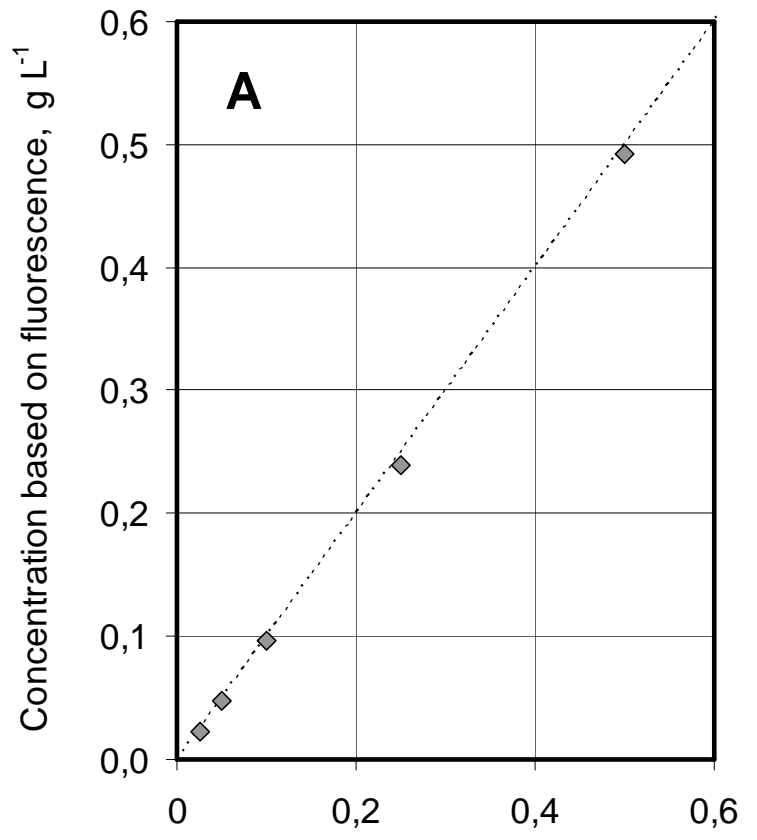

Expected concentration, $\mathrm{g} \mathrm{L}^{-1}$

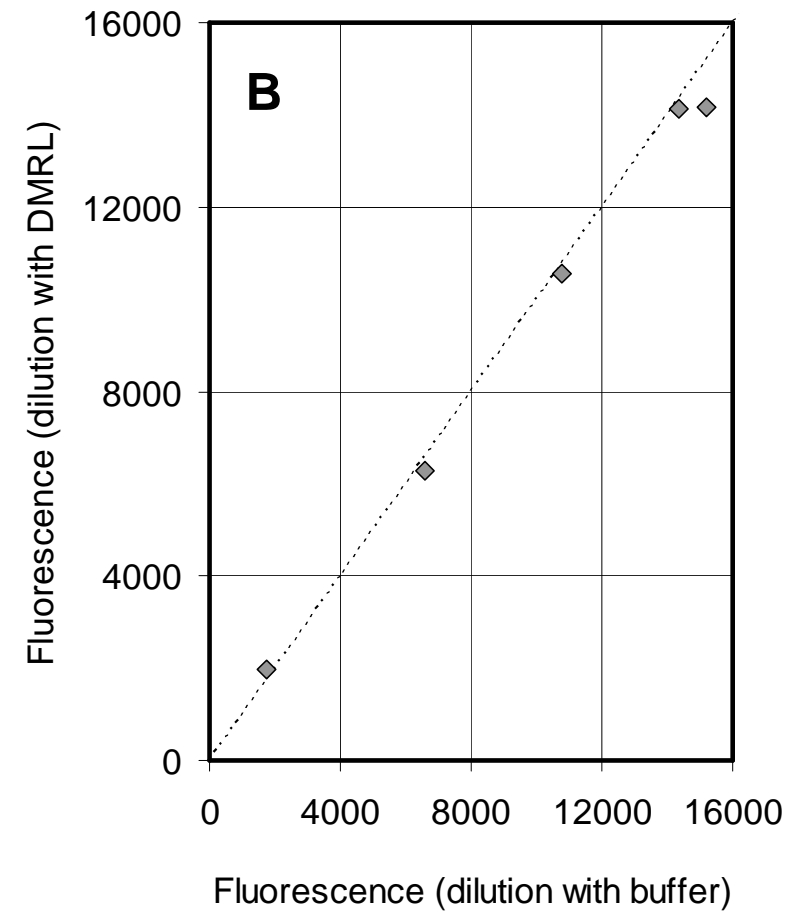

Figure 4-31: Assessment of the influence of di-methyl-ribityl-lumazine (DMRL) on the fluorimetric quantification of riboflavin in riboflavin standard solutions, diluted 1:2 with DMRL solution of approximately the same concentration (A), or in B2 process samples, diluted 1:2 with the same DMRL solution (B). Excitation took place at $485 \mathrm{~nm}$. The dotted lines (slope $=1$ ) indicate no influence of DMRL.

In terms of possible disturbance of the riboflavin quantification by other components in the B2 process samples, the influence of the presence of biomass in diluted samples was examined. The investigations with samples that were diluted $1: 36000$ revealed that there was no significant difference in riboflavin detection between samples with or without separated cell mass (data not shown).

Up to this point, all of the investigations were carried out manually using micropipettes for the dilution steps. The liquid handling according to the developed dilution scheme was then transferred to the laboratory robot. Fresh process samples from B2 cultivations at laboratory scale were placed into sampling tubes in the milliliter setup. The initial aspiration from these tubes and the subsequent dilution steps were carried out by the laboratory robot in replicates of three and the samples were examined fluorimetrically at a final dilution of 1:36000. The resulting values for the riboflavin concentration were compared with the regular offline data of the process determined via absorbance or HPLC. The outcome for one process is displayed in Figure 4-32, revealing an overall agreement of the average values of the automated method with the reference values. However, the variation on the measurement was high, with standard deviations for triplicates up to $20 \%$. This 
was clearly worse compared to manually pipetted replicates with standard deviations generally $<5 \%$ (data not shown).

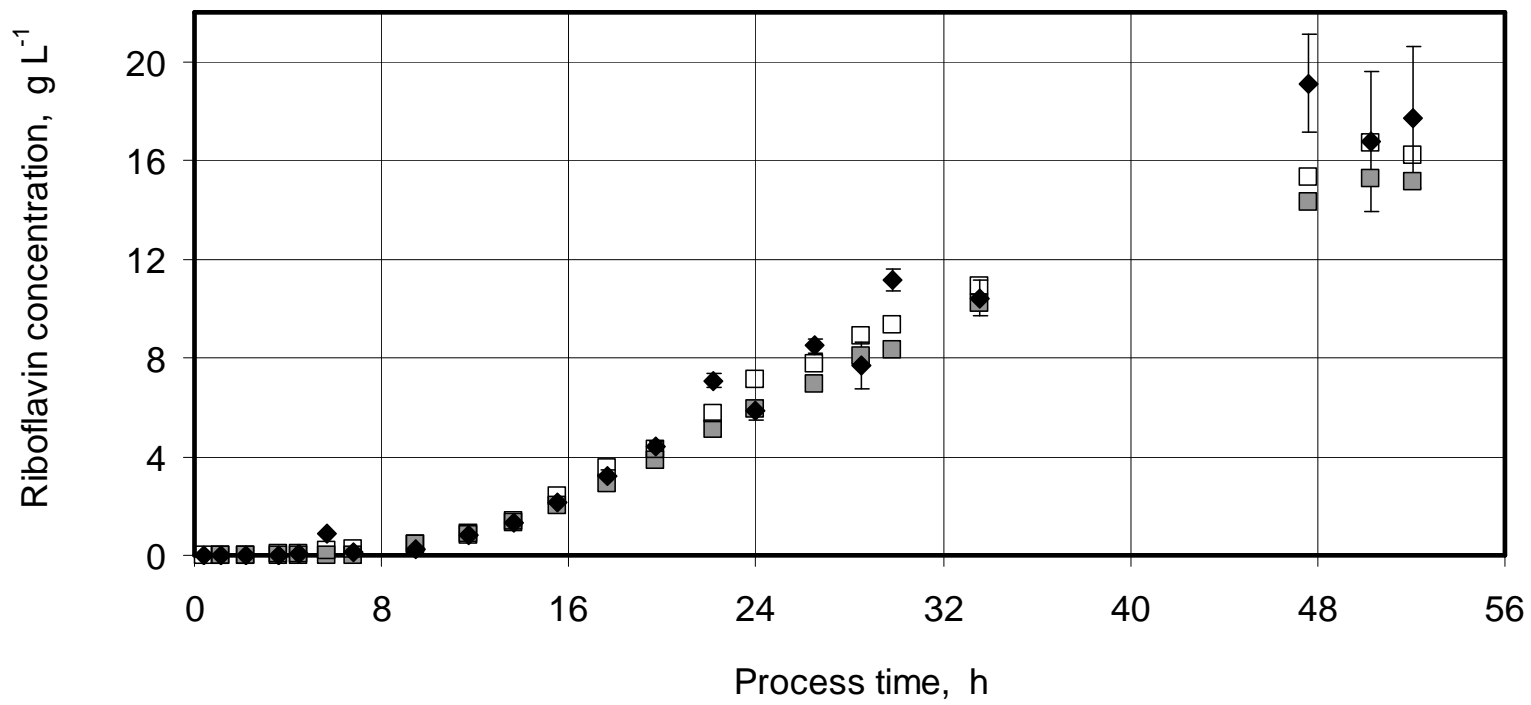

Figure 4-32: Riboflavin concentration during a B2 process at laboratory scale determined fluorimetrically in triplicates in the photometric plate reader after dilution with the laboratory robot (black diamonds), compared with the offline data measured via absorbance or HPLC (open or grey squares, respectively). Error bars are based on the standard deviation due to the liquid handling by the laboratory robot.

\section{Discussion}

All the presented data confirm that the fluorimetric quantification of riboflavin at $\lambda_{\text {excitation }}=485 \mathrm{~nm}$ was not significantly influenced by the presence of DMRL. The tendency of slightly lower values in riboflavin standard solutions or in B2 process samples containing DMRL could most likely be attributed to the dilution procedure. Using $485 \mathrm{~nm}$ instead of $440 \mathrm{~nm}$ for the excitation during the fluorimetric determination of riboflavin, and thereby avoiding the influence of DMRL, represented an elegant selective analytical method for this process. It required very little effort and equipment compared to a HPLC analysis. In addition, the comparison of the fluorescence values from measurements with either excitation wavelength enabled the assessment of process samples for the possible presence of DMRL or other by-products of the riboflavin synthesis that would be excited to fluoresce at $440 \mathrm{~nm}$, but not at $485 \mathrm{~nm}$.

The stated measuring range of $0.05-0.5 \mathrm{mg} \mathrm{L}^{-1}$ riboflavin could easily be expanded toward larger values, if necessary, by using a non-linear correlation. Furthermore, values below $0.05 \mathrm{mg} \mathrm{L}^{-1}$ could be detected, but were considered not fully reliable. The dilution scheme also allowed adjustment to higher final dilution to be used for samples containing even greater riboflavin concentrations. 
The variation on the fluorimetric determination of replicate samples was very high when the liquid handling was carried out by the laboratory robot. To address this, additional offline analyses for the product detection were performed at regular times during automated B2 processes in the milliliter setup, to guarantee a reliable characterization of the process performance. Sampling for these analyses took place at least every 24 hours.

\subsubsection{Summary}

For at-line monitoring of the B2 processes at the milliliter scale, the biomass, glucose and riboflavin concentrations could be assessed photometrically using only one $20 \mu \mathrm{L}$ sample from each reactor ("all-in-one" analysis). A separation of cells from the samples was not necessary. The analysis of up to 48 parallel $\mathrm{mL}$-reactors could be carried out quasi-simultaneously, fully automated with the laboratory robot, using four dilution steps in two 96-well-microtiter plates and a photometric plate reader, which could measure absorbance and fluorescence.

The dry cell mass $c_{X}$ could be estimated after the first dilution step with $\mathrm{NaOH}$ and addition of citric acid (total dilution $=1: 18$ ) by measuring the optical density at $650 \mathrm{~nm}$. A polynomial correlation between $O D$ and $c_{X}$ allowed the determination of $c_{X}$ in a range of $2.5-50 \mathrm{~g} \mathrm{~L}^{-1}$.

Glucose could be detected after a reactive dye solution was added to the first half of the second 96-well-plate with a precision pump inside the photometric plate reader, which took place after three dilution steps carried out by the laboratory robot (total dilution $=1: 10800$ ). The employed enzymatic assay provided valuable qualitative results, which allowed the monitoring of the initial glucose consumption as well as the detection of possible glucose accumulations after elevated process time. However, an accurate quantitative assessment of the glucose concentration was not always possible.

The fluorimetric quantification of riboflavin with an excitation at $485 \mathrm{~nm}$ was not influenced by the presence of its biosynthetic precursor DMRL. Hence, an elegant riboflavin-selective analysis of process samples containing $2.5-27 \mathrm{~g} \mathrm{~L}^{-1}$ riboflavin was possible. Despite the low deviations of single pipetting steps, the accumulated errors during the "all-in-one" analysis were rather significant after the third and especially after the fourth dilution step (final dilution 1:54 000). This sabotaged the at-line determination of riboflavin during B2 processes at the milliliter scale because of deviations up to $20 \%$. Since the milliliter setup was intended to be used as a screening tool, the riboflavin analysis needed to provide reliable quantitative data with variations $<5 \%$ for a valuable comparison of the outcome of various B2 processes in different reactors. Therefore, instead of relying solely on 
the automated at-line analysis of riboflavin, dependable offline analyses via HPLC had to take place at least every 24 hours for the characterization of the individual process performances. The sampling for this procedure was integrated into the process schedule to be carried out up to every three hours. 


\subsection{Automated parallel pH control at the milliliter scale}

The 48-fold screening setup at milliliter scale did not include a method for online $\mathrm{pH}$ measurement, therefore the determination of $\mathrm{pH}$ values had to take place outside the vessels. The resulting at-line $p H$ values were used for a $p H$ control algorithm, which was applied during parallel B2 cultivations in the milliliter setup.

\subsubsection{Parallel $\mathrm{pH}$ measurement}

The parallel at-line $\mathrm{pH}$ determination of freshly taken process samples took place in HydroPlates ${ }^{\circledR}$. These are transparent round bottom 96-well microtiter plates with a $\mathrm{pH}$-sensitive fluorophore immobilized in each well, allowing the simultaneous assessment of up to 96 parallel samples of as little as 5-10 $\mu \mathrm{L}$. The fluorophore exhibits different intensities of fluorescence dependent on the $\mathrm{pH}$ of the solution in the well. A second $\mathrm{pH}$-independent fluorophore is co-immobilized in each cavity as a reference and allows the reduction of well-to-well variation. In commercially available "HP96L" HydroPlates ${ }^{\circledR}$ an additional dark polymer is part of the fluospot for shielding the $\mathrm{pH}$-dependent signal against disturbing foreign fluorescence (PreSens, 2003).

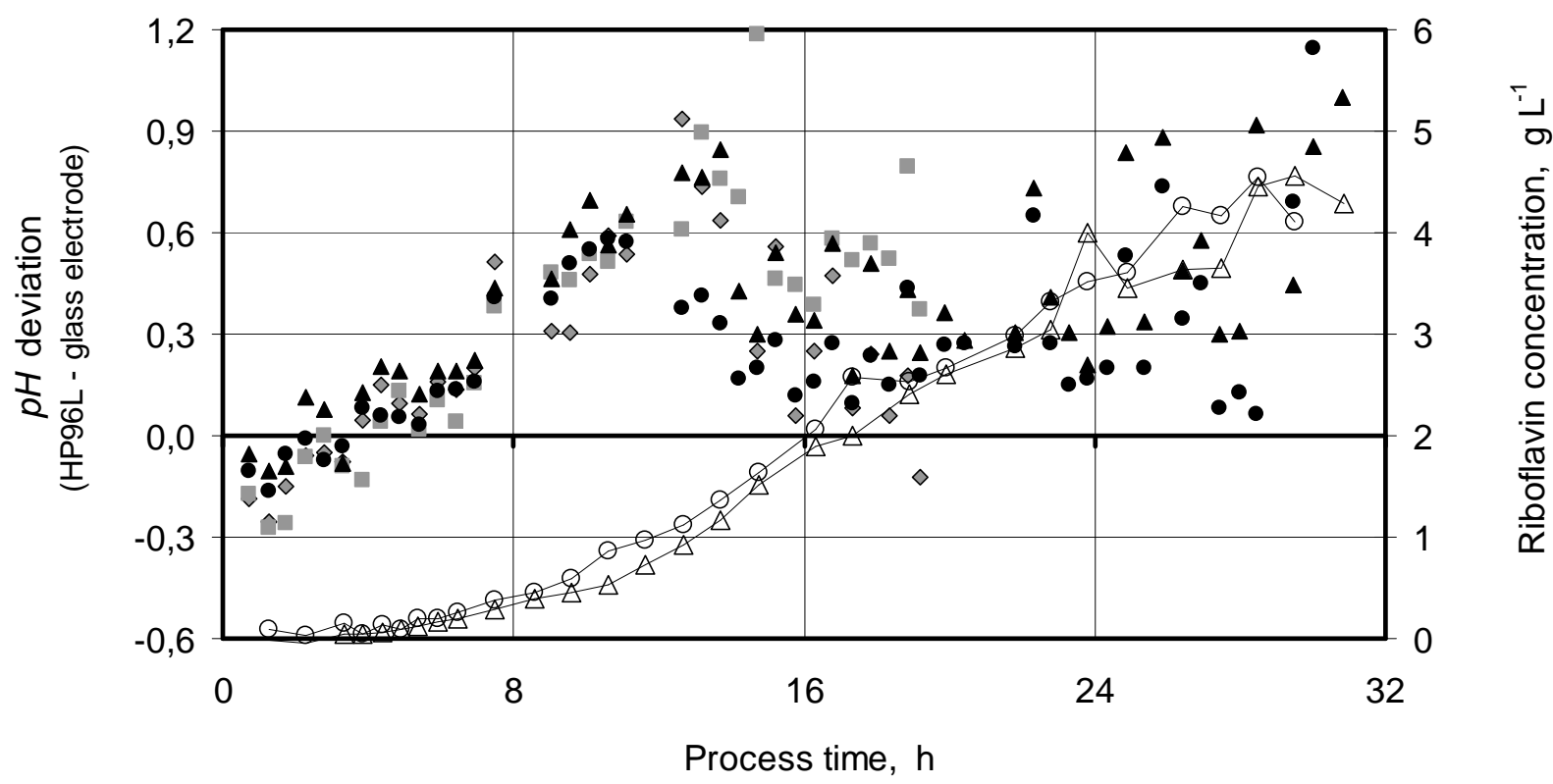

Figure 4-33: Deviations of the parallel $p H$ measurements in a "HP96L" HydroPlate ${ }^{\circledR}$ from the determinations with a miniature standard glass electrode during preliminary B2 processes in four parallel bioreactors in the milliliter setup (black and grey symbols) along with the riboflavin concentrations in two of these reactors (open symbols, connected with lines).

The excitation and emission wavelength for the indicator fluorophore in "HP96L" HydroPlates $^{\circledR}$ is the same as for riboflavin. As a result, the $\mathrm{pH}$ measurement of samples from the B2 process is significantly impaired, despite the optical shielding 
in these plates. The $\mathrm{pH}$ measured at-line during preliminary $\mathrm{B} 2$ processes was on average up to $0.8 \mathrm{pH}$ units higher than that determined with a miniature standard glass electrode, particularly between eight and 16 hours (Figure 4-33). The growing deviation was initially correlated to the increasing riboflavin concentration until approximately $1.0-1.5 \mathrm{~g} \mathrm{~L}^{-1}$. After that the $\mathrm{pH}$ determined with the HydroPlate ${ }^{\circledR}$ mainly strayed in a range of 0.2 to $0.6 \mathrm{pH}$ units above the values measured with the glass electrode.

In addition to the influence of riboflavin on the measurement, there was a drift of the $\mathrm{pH}$ determined in "HP96L" HydroPlates ${ }^{\circledR}$ during repeated measurements of reference buffers between $\mathrm{pH}=7$ and $\mathrm{pH}=8$ with the same plate over 30 hours (Figure 4-34). This was not observed when the HydroPlate was blackened from the outside for additional optical shielding between the wells (data not shown). However, the external blackening was very laborious and a commercial version of this treatment would presumably be very costly.

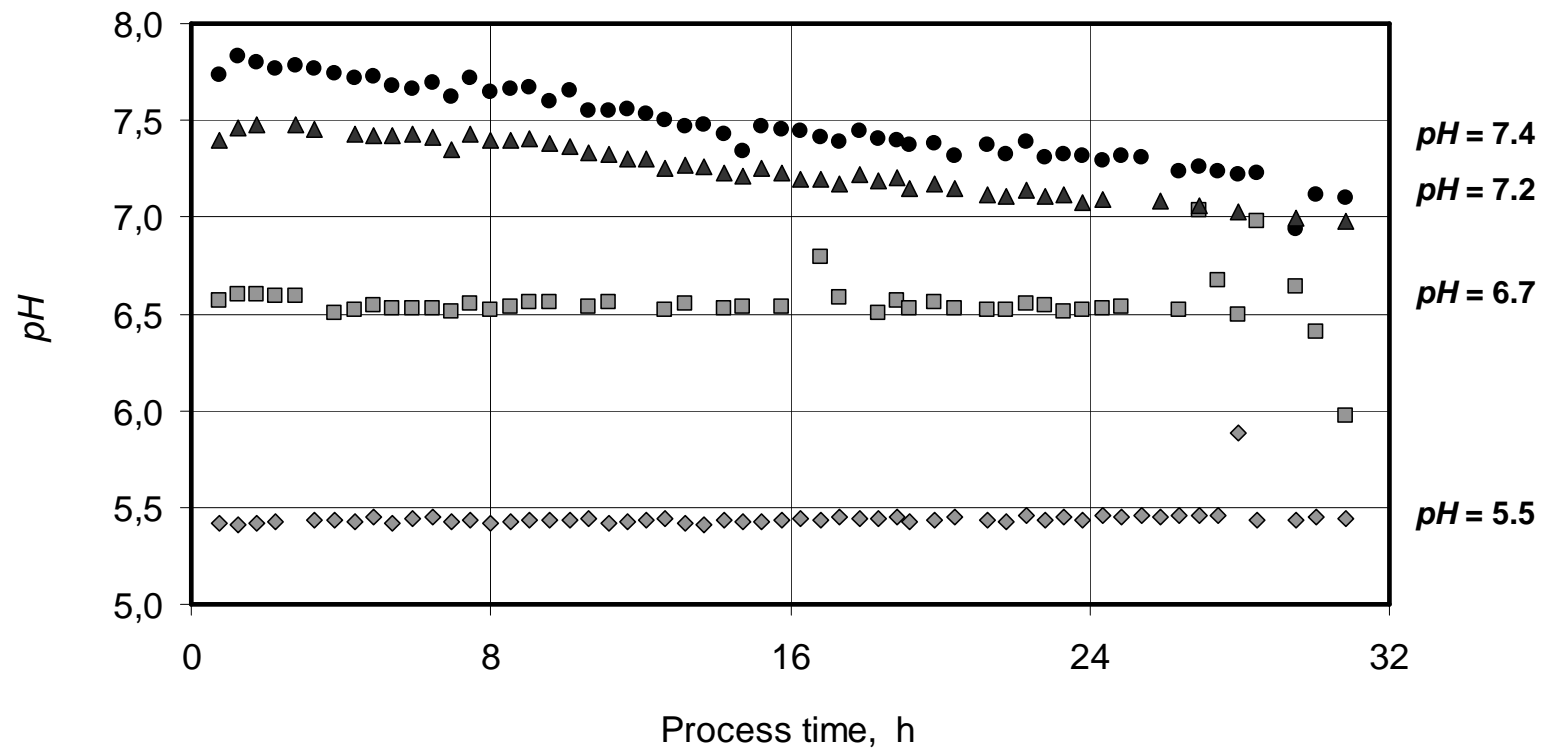

Figure 4-34: Repeated $p H$ measurements of four different reference buffers $(p H 5.5,6.7,7.2,7.4)$ in the same "HP96L" HydroPlate ${ }^{\circledR}$.

Numerous approaches for improving the performance of different HydroPlates ${ }^{\circledR}$ were undertaken, the most promising being external blackening of the wells or tests of HydroPlates ${ }^{\circledR}$ with "brighter" fluorophores. For details refer to Solf (2003). None of these attempts yielded satisfactory results for all the required aspects. Major weaknesses included extended response times due to thick shielding layers as well as poor reproducibility, mostly due to the manual fabrication of the fluospots because of commercial unavailability. 
For all of these reasons, the $p H$ values measured in "HP96L" HydroPlates ${ }^{\circledR}$ were accepted as "apparent" $\mathrm{pH}$ values and the deviations of these $\mathrm{pH}$ values from those determined with a glass electrode during the initial phases of several B2 processes at milliliter scale (data not shown) were used to develop apparent $p H$ setpoint profiles for the $\mathrm{pH}$ control in the milliliter setup (Figure 4-35). With these apparent setpoints the actual $\mathrm{pH}$ during the milliliter processes should be kept in a tolerable range for unaltered process performance. This range was at least $p H$ $=6.5-7.5$, which had been shown during B2 processes at laboratory scale.

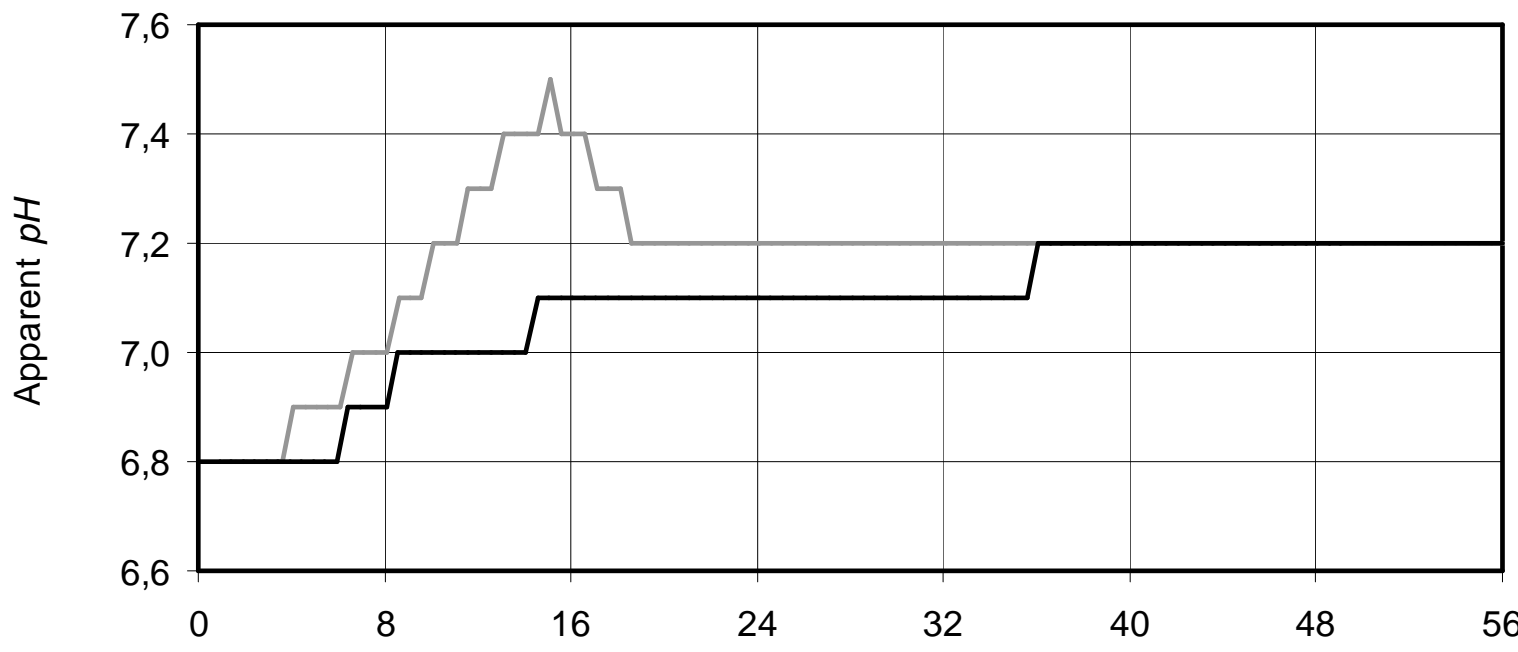

Process time, $\mathrm{h}$

Figure 4-35: Two apparent $\mathrm{pH}$ setpoint profiles tested for the $\mathrm{pH}$ control during $\mathrm{B} 2$ processes to compensate the influence of dissolved riboflavin when "HP96L" HydroPlates ${ }^{\circledR}$ were used for the $\mathrm{pH}$ determination. The course of the grey profile is stronger correlated to the deviations of $p H$ measurements in "HP96L" HydroPlates ${ }^{\circledR}$ from those with glass $p H$ electrodes.

There was no difference in the performance of B2 processes which were operated either with the $\mathrm{pH}$ setpoint profile that accounted for the dramatic $\mathrm{pH}$ deviations, observed between eight and 16 hours, with a steep rise and subsequent decrease, or with the setpoint profile with a moderate increase during this time (data not shown). Therefore, the more conservative $\mathrm{pH}$ setpoint profile without major shifts (black line) was selected for automated B2 processes in the milliliter setup.

With the expectation of different delay times between sampling and the actual $\mathrm{pH}$ measurement for 48 reactors during automated cultivations, the influence of the sample incubation time in the HydroPlate ${ }^{\circledR}$ on the $p H$ determination was tested. The $p H$ values were the same after no more than three minutes (data not shown). 


\section{Discussion}

Dissolved riboflavin was presumably the dominant source of the diverging $\mathrm{pH}$ values measured with "HP96L" HydroPlates ${ }^{\circledR}$ despite optical shielding in these plates. The deviations of the $\mathrm{pH}$ measurement started to decline at $1.0-1.5 \mathrm{~g} \mathrm{~L}^{-1}$ riboflavin, apparently because riboflavin started to crystallize at concentrations in this range due to its limited solubility. As a consequence the concentration of dissolved riboflavin most likely decreased slightly and remained below $1 \mathrm{~g} \mathrm{~L}^{-1}$ for the rest of the process.

Even though the use of HydroPlates ${ }^{\circledR}$ for the measurement of $p H$ values during B2 processes was not optimal, alternative methods suitable for the milliliter setup, for example the parallel application of multiple miniature glass probes or ion-selective field effect transistor (ISFET) sensors (Baumann et al., 1999), were either very costly or technically very complex. In addition, the ISFET technology needs a reference electrode for the $\mathrm{pH}$ determination and is generally afflicted with a considerable drift over several hours requiring regular adjustment (Brischwein, 2003). An interesting alternative for the at-line $\mathrm{pH}$ measurement could possibly be the use of magneto-elastic sensors that enable the remote query measurement of $p H$ from a passive, wireless platform, independent of temperature after an initial calibration, and with a response time (90\%) of less than three minutes (Jain et al., 2001; Yang et al., 2002). Ruan et al. (2003) reported a linear operating range of $\mathrm{pH} 3$ to $\mathrm{pH} 9$ with these thick-film coated sensors, independent of the present salt concentration. It is questionable, however, if these sensors would work reliably over several days in the automated setup and if they could be made available in a parallel composition feasible for integration on the robot's worktable.

Since the approaches for reducing the influence of riboflavin on the $\mathrm{pH}$ measurement in various tailor-made HydroPlates ${ }^{\circledR}$ were also not convincing, the application of the "HP96L" HydroPlate ${ }^{\circledR}$ for the assessment of the $p H$ represented the method of choice with the fewest draw-backs. The major advantages of this HydroPlate $^{\circledR}$ were that it was commercially available and the measurements were simple, reliable and reproducible. The response time between $\mathrm{pH}=5$ and $\mathrm{pH}=9$ was less than three minutes, values between $p H=6$ and $p H=8$ equilibrated clearly faster, so that consistent circumstances for the simultaneous $\mathrm{pH}$ measurement of 48 samples in this range could be anticipated. The possible drift of $p H$ $>7$ after repeated measurements in the same HydroPlate ${ }^{\circledR}$ may act against the influence of riboflavin. For a reliable $\mathrm{pH}$ assessment it should be avoided to use the same wells of a "HP96L" HydroPlate ${ }^{\circledR}$ for more than 24 hours.

In combination with the apparent $p H$ setpoint profile, the use of the "HP96L" HydroPlate ${ }^{\circledR}$ for the $p H$ control during the B2 processes at milliliter scale should be 
possible, because this process seemed very robust with respect to variations of the $p H$, as discussed in section 4.3.3. However, this method may not be valid for other processes which are more sensitive to varying $\mathrm{pH}$ value and during which the optimal process performance depends crucially on an accurate measurement and adjustment of the $\mathrm{pH}$. Furthermore, this approach requires knowledge about the quantitative impact of the disturbance.

\subsubsection{Resulting $\mathrm{pH}$ during $\mathrm{B} 2$ processes in the milliliter setup}

The resulting $\mathrm{pH}$ profile during 48 automated parallel B2 processes in the milliliter setup is depicted in Figure 4-36. During this experiment the conditions were the same for all 48 vessels to prove parallel reproducibility. Additions of $12.5 \%$ ammonium hydroxide every ten minutes were used for $\mathrm{pH}$ adjustment, based on $\mathrm{pH}$ values measured every 30 minutes.

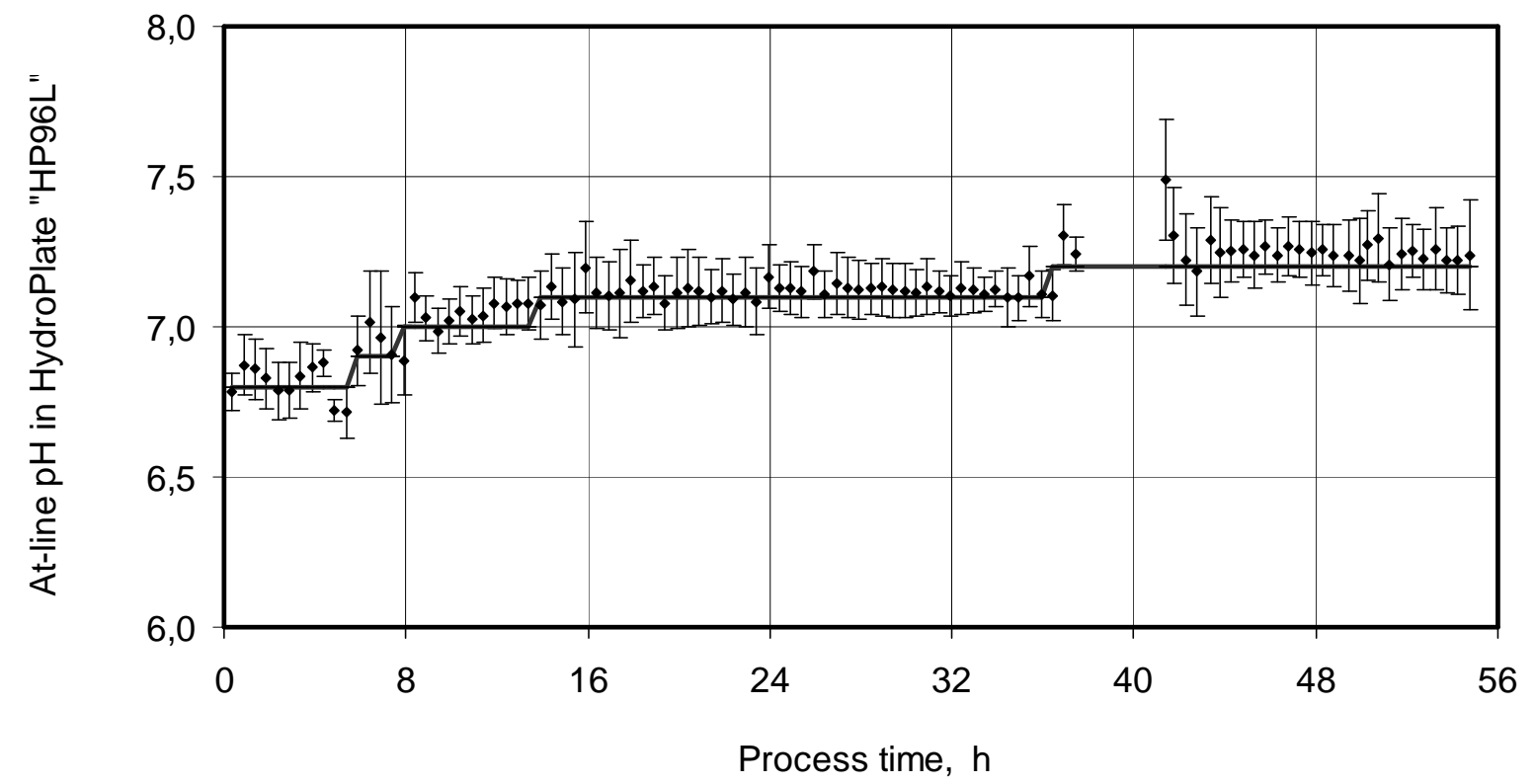

Figure 4-36: Average $\mathrm{pH}$ during 48 parallel $\mathrm{B} 2$ processes at milliliter scale according to the intermittent at-line measurements in "HP96L" HydroPlates ${ }^{\circledR}$ (symbols) compared with the predetermined apparent $\mathrm{pH}$ setpoint profile (line). The error bars are based on the standard deviation of initially all 48 reactors. After 38 hours the averages are based on only the 28 reactors which still ran properly after a complete process downtime of 3 hours.

The displayed $\mathrm{pH}$ values are initially based on the average of all 48 reactors. After 38 hours the averages are based on only the 28 reactors which still ran properly after a complete process downtime of three hours. The intermittently measured $\mathrm{pH}$ values remained close to the apparent $\mathrm{pH}$ setpoint profile and the standard deviations were $<0.11 \mathrm{pH}$ units or $<2 \%$ during the whole process. 


\section{Discussion}

A measurement every 30 min could not reveal any of the $p H$ fluctuations which took place between the pulsed feed additions every $10 \mathrm{~min}$. This is only possible with an online determination. Preliminary experiments with $\mathrm{pH}$-sensitive fluospots immobilized inside the milliliter reactors indicated similar oscillations to those observed at the laboratory scale (data not shown). The required technology for this type of online $\mathrm{pH}$ measurement in the milliliter setup is currently under development at PreSens (Regensburg, Germany). As this technology is based on a principle similar to the $\mathrm{pH}$ determination in HydroPlates ${ }^{\circledR}$, the potential influence of dissolved riboflavin on this measurement will also have to be considered.

The $\mathrm{pH}$ values obtained with the intermittent at-line measurement in "HP96L" HydroPlates ${ }^{\circledR}$ were very close to the respective setpoints. However, with this method it was not completely clear how much the present riboflavin or a possible drift of the signal influenced the data quantitatively and therefore what the real $p H$ values were.

\subsubsection{Summary}

The automated parallel $\mathrm{pH}$ measurement at the milliliter scale could be carried out at-line with commercially available "HP96L" HydroPlates ${ }^{\circledR}$. As the measurement was influenced by the dissolved riboflavin in the samples, a preset apparent setpoint profile had to be used for the $\mathrm{pH}$ control algorithm. The resulting $\mathrm{pH}$ profiles for 48 parallel $\mathrm{B} 2$ processes based on the intermittent $\mathrm{pH}$ measurement and control every 30 minutes followed the $p H$ setpoint profile within approximately \pm 0.2 $\mathrm{pH}$ units. The standard deviation was $<0.11 \mathrm{pH}$ units during the whole process, making the developed intermittent $\mathrm{pH}$ control an acceptable tool for the B2 process under the given circumstances. 


\subsection{Automated parallel B2 processes at the milliliter scale}

This section commences with the introduction of the schedule developed for all the actions necessary during automated parallel cultivations. Subsequently, the approaches for aseptic operation of the system and the handling of foam suppression are presented and discussed. This is followed by a subsection that considers some of the physical and chemical conditions that had an influence on the process performance, namely the dissolved oxygen together with the phenomena associated with the gas input into the system and the volumetric increase of the medium during the feeding phase. Based on these prerequisites for operating the B2 process in the described milliliter setup, the performance of parallel B2 cultivations is presented with respect to glucose consumption, growth and productivity of $B$. subtilis and the reproducibility of these features as the major outcome of this project. The summary at the end highlights the most important results considering all the aspects mentioned in this section.

\subsubsection{Scheduling the automated actions for 48 parallel B2 processes}

There were several activities that the laboratory robot had to perform during the B2 processes in the milliliter setup. Firstly, the most crucial activities were the intermittent feed addition, the $\mathrm{pH}$ measurement and adjustment, and the suppression of foam buildup for maintaining the essential process operation. Secondly, the sampling and sample preparation required extensive additional actions of the liquid handling system because every sample had to be diluted four times for complete monitoring of the process parameters glucose, biomass, and riboflavin concentration. The durations for all the sampling and dilution steps were measured as well as for the liquid additions, when either the "single-pipetting" or the "multi-pipetting" mode of the laboratory robot was used. The "multi-pipetting" mode saved time by aspirating the volume of a fluid to be added to a complete row of reactors with one needle and dispensing the individual portions to the reactors without washing steps in between.

Based on the measured durations, a schedule for the automated operation of parallel B2 processes in up to 48 milliliter bioreactors was developed. It was organized in cycles of three hours with equal actions (Figure 4-37). Every three hour cycle was divided into six 30 min units, of which each one was ruled by the essential "heartbeat" of feed and base addition every $10 \mathrm{~min}$. The $\mathrm{pH}$ measurement and its feedback for the calculation of the necessary base volumes always took place during the first $10 \mathrm{~min}$ of a $30 \mathrm{~min}$ unit. The $\mathrm{pH}$ setpoint profile for the $\mathrm{pH}$ control algorithm was automatically read from a preset profile. During the same $10 \mathrm{~min}$ unit an automated antifoam addition was performed. The remaining time slots 
during the second and third 10 min interval were used for sampling from the vessels for process monitoring of biomass, glucose and riboflavin concentration every three hours, and for the multiple steps of the sample dilution. Additionally, $20 \mu \mathrm{L}$ samples were taken from the vessels automatically every 24 hours for offline measurements (HPLC and absorbance). Alternatively, the offline sampling could take place up to every three hours. All the described actions were performed quasi-simultaneously for all the parallel reactors. The addition of fluids to the reactors was carried out with the "multi-pipetting" mode of the robot, because using the "single-pipetting" mode resulted in times up to six minutes for the dispensing of the feed or the base solution to six rows of reactors, thus in more than ten minutes for both additions combined.

\section{Process control}

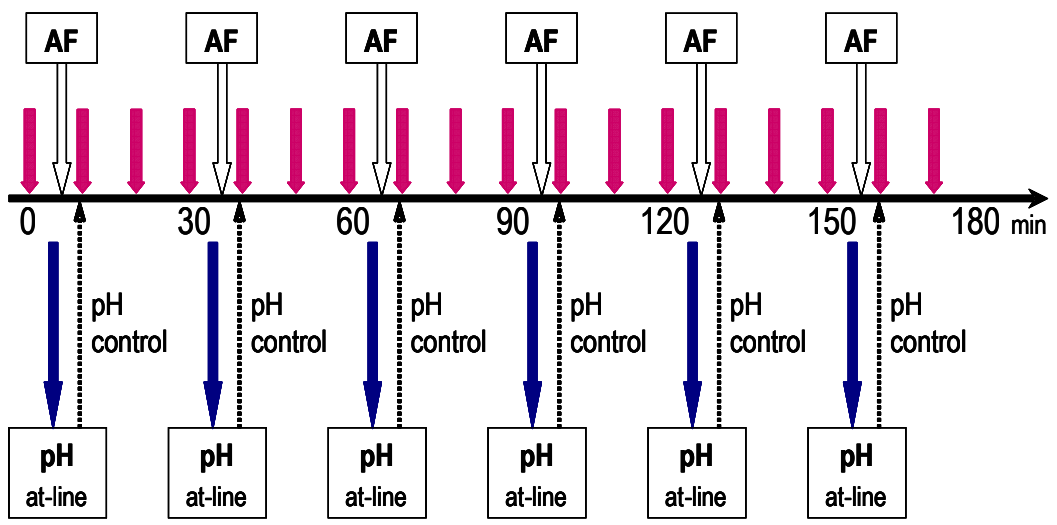

Process monitoring

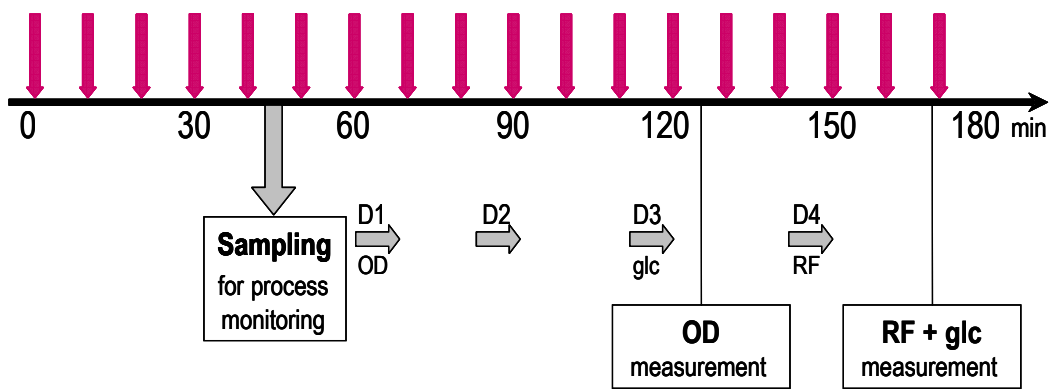

Figure 4-37: Schedule for the automated operation of parallel B2 processes in up to 48 stirred tank bioreactors in the milliliter setup. Vertical grey arrows indicate the addition of feed and base ("heartbeat"). Abbreviations: AF, antifoam; D1- D4, dilution steps 1-4, respectively; $O D$, optical density; RF, riboflavin concentration; glc, glucose concentration.

GEMINI, the laboratory robot control software, was used as the executive software for the overall control of the automated parallel B2 processes at milliliter scale. Software routines, which had been programmed in LabVIEW (Puskeiler et al., 2005a), allowed the communications of the robot with the plate reader and the plate washer. Further LabVIEW routines handled the evaluation of the $\mathrm{pH}$ data 
for calculating the individual base volumes that had to be added to each milliliter vessel with the laboratory robot. The feeding process could be started or stopped at any point of time in the process for each individual reactor (optional volumes). It restarted at the beginning of the next 10 min cycle, while the schedule of the interlaced actions for the process analyses remained undisturbed. This was also done by employing independent LabView routines for the generation of files, which contained the respective liquid volumes and could be directly used by the laboratory robot. The antifoam addition every $30 \mathrm{~min}$ followed the same principle.

\section{Discussion}

The dilution steps had to be allocated a significant amount of time within the schedule in order to achieve a precision as high as possible, with variations as low as possible even after the last dilution step. Additionally, the addition of feed had to be very accurate, hence lasting rather long as well.

Combining the various actions resulted in a very tight schedule for the 48-fold parallel experiments. It made the use of the "multi-pipetting" mode of the lab robot necessary for the addition of feed, base, and antifoam solutions. While each of the eight robotic needles subsequently served six parallel reactors in one row of the reaction block with this mode, it did not include a washing step between dispensing fluids to the different columns of reactors ${ }^{3}$. As a consequence, this configuration allowed the use of the milliliter setup for a maximum number of eight different strains (one per robotic needle) when the transfer of different clones between vessels of the same row due to aerosols in the headspace of the reactors should be avoided.

\subsubsection{Aseptic operation of the milliliter setup}

The reaction block is not a closed system. Channels in the cover lid above each reactor allow access of the robotic pipettes to every vessel for liquid additions into the headspace or for sampling. Furthermore, the gas flow for the aeration of the processes escapes the milliliter reactors via these channels, which presumably implies that no particles from the outer environment can enter the vessels.

Nevertheless, a critical aspect for the operation of the milliliter setup was the occurrence of foreign contaminations. These could initially originate from the use of non-disposable milliliter vessels which were not irradiated for sterilization but

\footnotetext{
${ }^{3}$ For a schematic display of the six parallel columns and eight parallel rows in the reaction block refer to Figure 7-8 on page 151.
} 
were only treated with ethanol and sodium hydroxide solutions prior to a cultivation. Contaminations could also be introduced by the robotic pipettes via the input of microbes originating either from the air or from the fluids stored in liquid containers on the worktable. Since the complete setup was not integrated in a sterile environment, the feasibility of keeping these reservoirs aseptic throughout a whole cultivation was rather limited.

Therefore, chloramphenicol was added to the batch medium of the B2 cultivations in order to avoid the growth of external contaminants. Nevertheless, foreign bacteria were regularly observed during the B2 processes even when sterile disposable reactors were employed and all the used fluids and their containers were sterilized before the start of a process. These bacteria were examined under the microscope and were found to sporulate and to be rod-shaped similar to the used B. subtilis, but significantly larger (Figure 4-38 A).
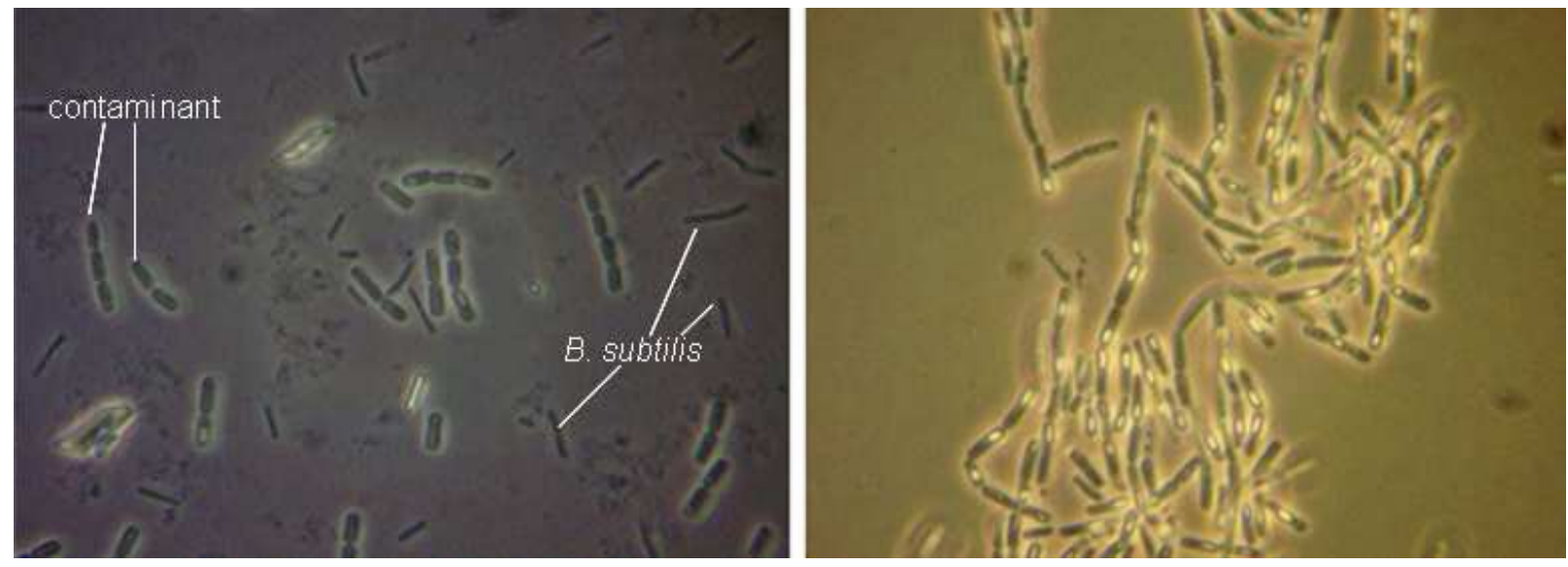

Figure 4-38: Microscopic photographs of the contaminating bacteria. A, contaminating bacteria together with B. subtilis; $\mathrm{B}$, generation of endospores by the contaminant. The spores can be clearly detected as bright condensed regions at one end of each bacterial cell. The cells had been grown on agar plates at $37^{\circ} \mathrm{C}$ for two days and were then stored at 4$7^{\circ} \mathrm{C}$ for three days to induce sporulation.

Their occurrence in different reactors was partially random and could not be correlated to a single row or column of the reaction block. There were always some reactors during 48-fold parallel cultivations which were completely free of sporeforming cells. The respective seed stocks for the cultivations were tested for contamination and were found to be clean of any foreign bacteria.

Besides foreign contaminations with external microorganisms, the possibility of transfer contaminations between neighboring reactors of the milliliter setup was examined under the actual conditions of the automated B2 process. Different washing procedures were applied, combining solely rinsing, or rinsing and resting in sodium hydroxide and/or ethanol or ammonium hydroxide, or in a mixed solu- 
tion of sodium hydroxide and ethanol. For a detailed description of the applied procedures and individual results refer to Biehl (2004).

Only the combined treatment with base and alcohol seemed successful. This disinfection step was applied during a control experiment and could suppress the transmission of bacteria from one column of inoculated reactors to two columns of sterile disposable vessels, which only contained growth medium, over a period of 12-16 hours or even until the end of the process after 45 hours (Figure 4-39). Only in one reactor did growth occur already after eight hours. The solution for disinfection was exchanged only once after approximately 24 hours.

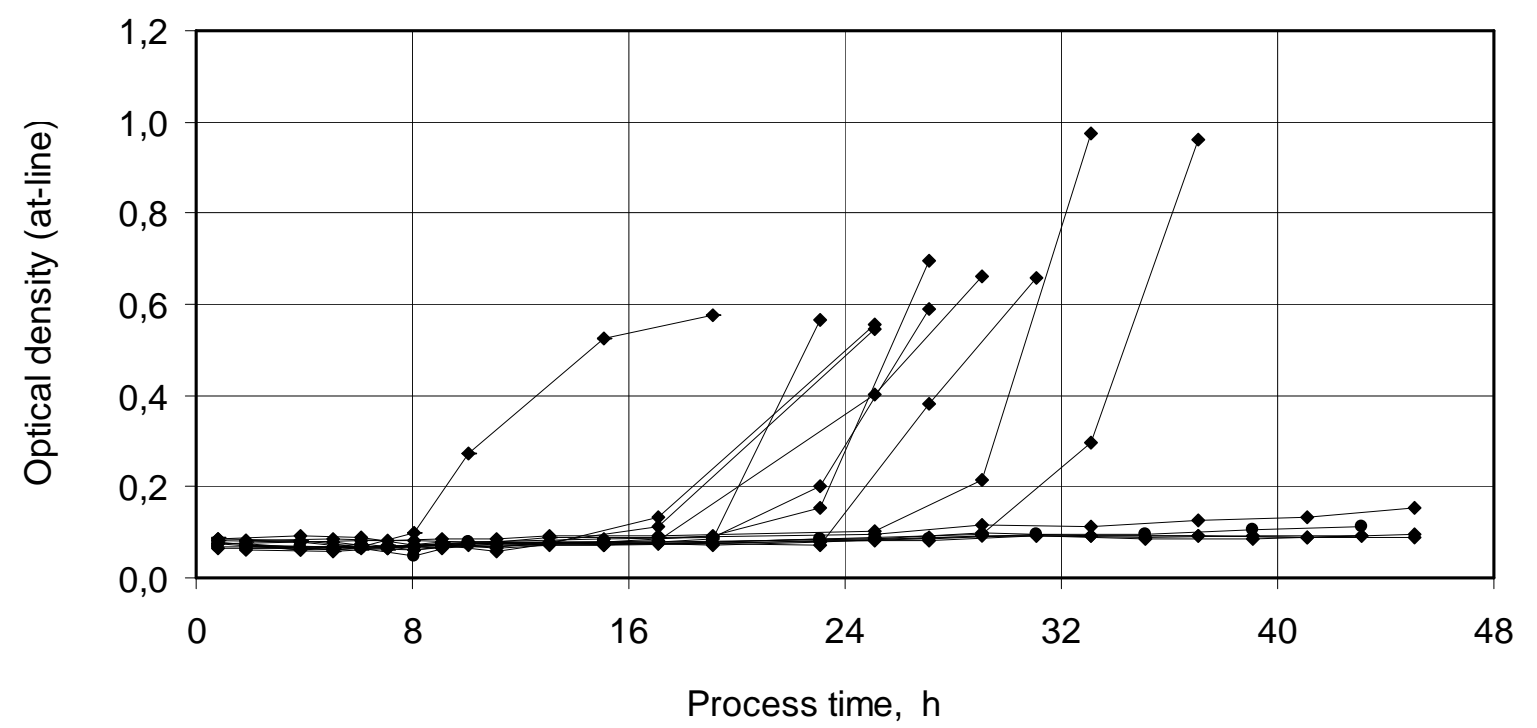

Figure 4-39: Optical density in 16 sterilized disposable reactors with medium as controls in two columns of the reaction block during investigations regarding the occurrence of transfer contaminations. The robotic needles primarily served a third column of parallel reactors with inoculated B2 processes and were washed between the actions of liquid handling in a solution containing $0.75 \mathrm{M}$ ammonium hydroxide and $70 \%$ ethanol. Every other hour samples were taken from either one of the columns with the control vessels. An increase of the optical density indicated growth in the respective reactor.

\section{Discussion}

Puskeiler (2004) demonstrated that the milliliter system could be operated aseptically over three days when no liquid handling was performed by the laboratory robot or when the robotic pipettes were treated with disinfecting washing steps between each pipetting step. However, the pipetting frequency during Puskeiler's experiments was considerably lower compared to the conditions during the automated B2 process and the concentration of $B$. subtilis cells in the aerosol in the reactor headspaces, and most likely in the gas flow leaving the cover lid, was higher compared to Puskeiler's experiments with E. coli. This made transfer contaminations between neighboring reactors during parallel B2 processes more probable to occur. Furthermore, the tight schedule for 48-fold B2 processes did 
not allow the more extended washing between sampling steps, which would be necessary to suppress transfer contaminations over an extended period of time.

Since the limited availability of time also required the "multi-pipetting" mode for all the liquid additions, the exchange of cells between reactors, which were served with the same robotic needle, had to be accepted anyway. Hence no elaborate washing procedures were used during the 48-fold parallel B2 cultivations, but only rinsing with water took place between the actions of the liquid handling system. This was not a problem as long as the use of the system was restricted to eight different clones of a bacterial strain, of which each one would be cultivated in a separate row so that it was served only by one robotic needle. Consequently, also the liquid containers for additive solutions needed to provide separate compartments for each needle.

The origin of the observed contaminations with sporulating bacteria could not be clarified completely. The initial concentration of chloramphenicol in the medium may have prevented foreign bacteria from growing in the beginning of a cultivation. However, as the resistance of the employed $B$. subtilis against chloramphenicol is based on the inactivation of this molecule by an acetyl-transferase (Schlee and Kleber, 1991), the amount of active antibiotic in the medium was most likely gradually reduced during the processes. This would have made it easier for foreign microbes to accumulate, particularly when they existed as durable endospores. During other cultivations in the same setup, carried out by different collaborators, no such contaminations occurred. Therefore, it is more likely that the spores resided in solutions or in components of the milliliter setup that were only used for the B2 process.

\subsubsection{Automated antifoam addition}

Foam formation was observed at elevated times during B2 processes at laboratory scale as well as in the milliliter setup. In the milliliter setup an automatic detection of this phenomenon was not possible. A thick layer of foam could impair the mass transfer between the liquid and the gas phase, and in extreme cases it could even leave the reactors through the access channels for the robotic needles. Since an increased supply of antifoaming agent to the initial batch medium did not render foam suppression lasting throughout the whole process, it was necessary to investigate how much foam-suppressing agent had to be added to the reactors during the course of a cultivation by the liquid handling system to avoid any critical foam buildup.

The major problem with the automated addition of antifoam solutions by the laboratory robot was the viscous and poorly water soluble character of these agents. 
Several different diluted or undiluted antifoam solutions "AF 1-4" were tested. "AF 1" contained organic antifoaming compounds and a small percentage of silicone for improved performance. This solution was used during B2 processes according to the original protocol. The three further solutions were based on polyalkoxyethers and did not contain any silicone. They were chosen based on the manufacturer's recommendations with respect to their suitability for fermentation (low cell toxicity), their solubility in water, and the possibility for sterilization. None of them should reduce the dissolved oxygen concentration in the medium upon addition to the process.

Testing the stability of emulsions in water of these antifoaming agents revealed increasing stability from "AF 1 " to "AF 4". The foam prevention or reduction, on the other hand, was most effective with "AF 2 " during first experiments, followed by "AF 3". However, the feasibility of both "AF 2" and "AF 3" for being pipetted by the laboratory robot, particularly with the "multi-pipetting" mode, was really poor. This became evident during a 48-fold parallel cultivation, during which several reactors received hardly any antifoam solution at all.

For this reason, "AF 4" diluted 1:4 with water was used. This emulsion was stable for at least twelve hours and could be handled reliably by the laboratory robot. The necessary amount of the emulsion to be added was $10 \mu \mathrm{L}$ per reactor every 30 min, beginning six hours after the process was started. This could be lowered to 2-5 $\mu \mathrm{L}$ after 40 hours, resulting in a total volume of added antifoam solution of approximately $700-800 \mu \mathrm{L}$. With this strategy 48 parallel B2 processes in the milliliter setup were operated without any problems regarding foam formation.

\section{Discussion}

The clear advantage of using a diluted antifoam solution and adding a larger volume of it for sufficient efficacy was a higher accuracy of the addition and thus a more reliable suppression of foam formation. The extra volume of water dispensed to the vessels due to the dilution could be compensated by the evaporation from the milliliter bioreactors.

\subsubsection{Dissolved oxygen in the milliliter bioreactors}

On-line monitoring of the dissolved oxygen DO was carried out optically with a prototype of an eightfold sensor unit in the first column of the reaction block. The instrument could externally detect the signals from $D O$-sensitive fluospots which were immobilized internally at the bottom of the milliliter vessels. This allowed the adjustment of the oxygen content in the gas flow based on the dissolved oxygen. 
Figure 4-40 displays the dissolved oxygen profiles of eight reactors in the first column during nine hours in the beginning of a 48 -fold parallel B2 cultivation as well as the oxygen fraction in the inlet gas flow. This was raised from $21 \%$ to $35 \%$ after six hours and up to $51 \%$ at 8.7 hours, which was done roughly according to the previously established profile for oxygen addition, but was mainly based on the actual dissolved oxygen signals. The goal was to avoid the increase of the oxygen fraction as long as possible, in order to prevent unnecessarily high maximum dissolved oxygen values, while guaranteeing sufficiently high minimum dissolved oxygen values at the same time. During later phases of the processes in the milliliter setup, oxygen fractions up to $60 \%$ were used.

Towards the end of the batch phase, between 4.5 and 5.5 hours for the process displayed in Figure 4-40, characteristic fluctuations of the dissolved oxygen occurred within only a few minutes difference for all eight reactors. This behavior was also observed for other cultivations around the same time (data not shown). At $5.15 \mathrm{~h}$ the intermittent feeding was started for all 48 reactors of this experiment.

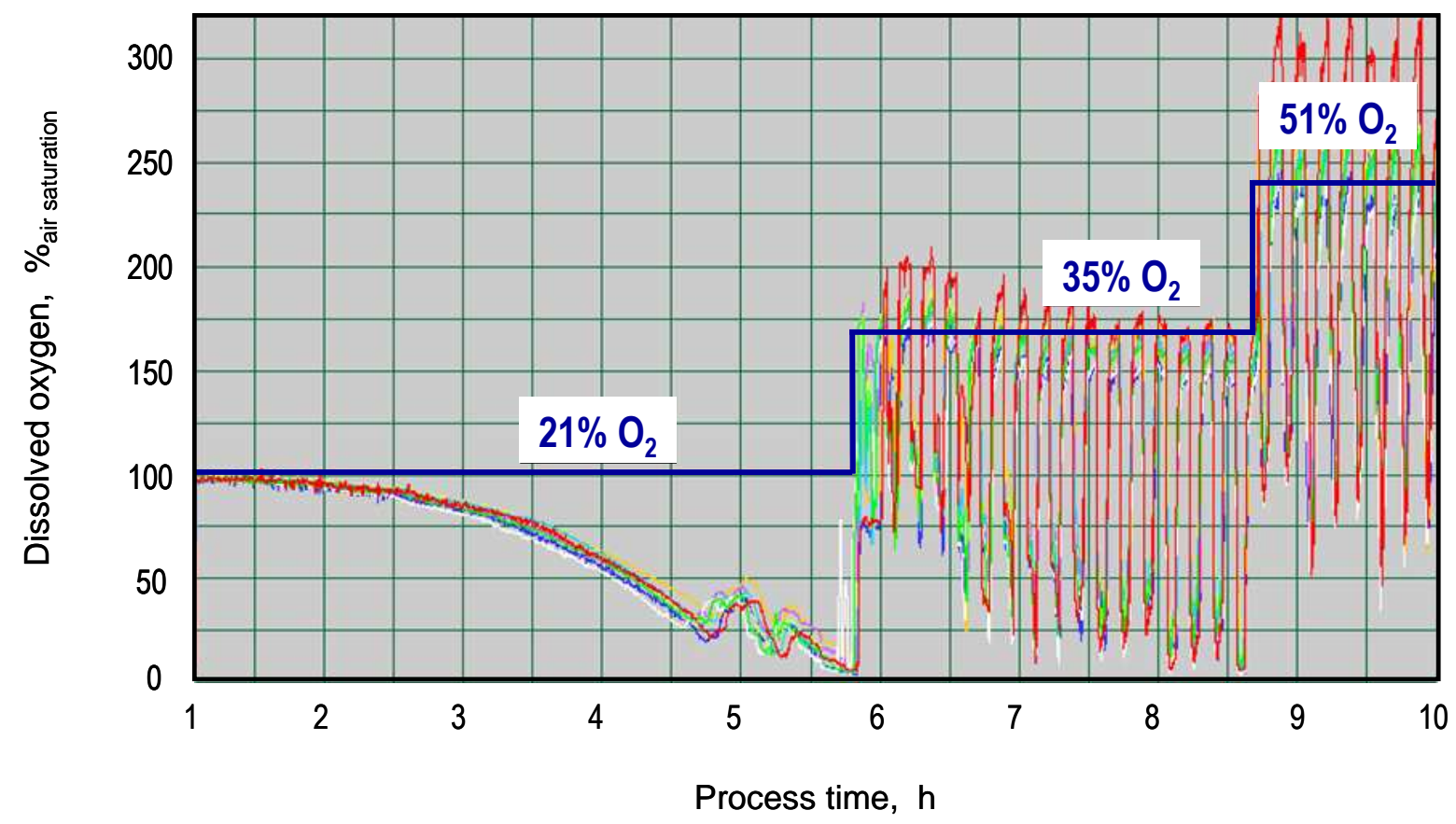

Figure 4-40: Dissolved oxygen during nine hours in the beginning of B2 processes in eight of 48 parallel milliliter bioreactors determined with an optical eightfold sensor unit. At $5.15 \mathrm{~h}$ the intermittent feeding was started. The oxygen content in the gas flow was raised from $21 \%$ to $35 \%(5.7 \mathrm{~h})$ and then to $51 \%(8.6 \mathrm{~h})$, indicated by the bold line and numbers. At $6.1 \mathrm{~h}$ the antifoam addition was started ( $10 \mu \mathrm{L}$ every $30 \mathrm{~min})$.

Whenever the carbon sources were completely metabolized, the dissolved oxygen rose steeply. It declined again once a new feed pulse was added. This lead to the characteristic oscillations of the dissolved oxygen, which had already been observed during the intermittent B2 processes at laboratory scale. The dissolved 
oxygen dropped additionally up to 30-50\% after each addition of $10 \mu \mathrm{L}$ antifoam solution every $30 \mathrm{~min}$, which was started at $6.1 \mathrm{~h}$.

During initial experiments in the milliliter setup some of the turbines stopped running after elevated process time, at approximately 20 hours. As a consequence of the interrupted agitation, the gas input into the respective reactor ceased and thus the process performance in this vessel was not successful, mainly with respect to the riboflavin generation (data not shown). The stoppage of stirring could be avoided after both the turbines and the central hollow axles were manufactured industrially and the power of the magnetic drive of the reaction block was reinforced.

$\mathrm{t}=6.0 \mathrm{~h}$; before the first antifoam addition

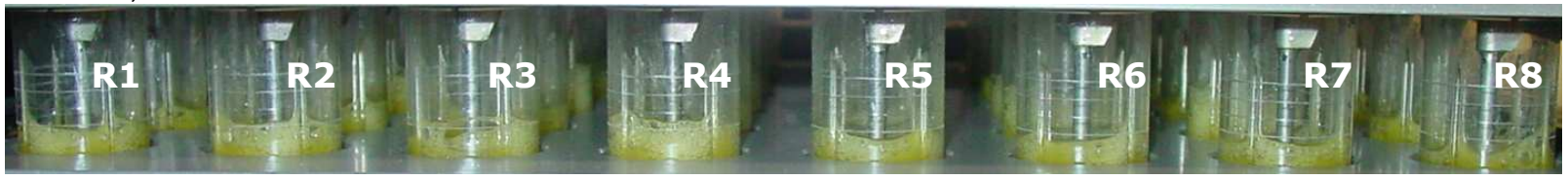

$\mathrm{t}=12.8 \mathrm{~h}$

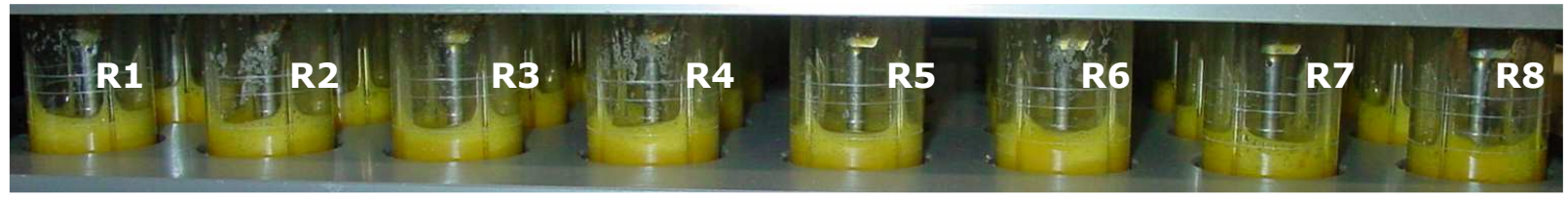

$\mathrm{t}=18.9 \mathrm{~h}$

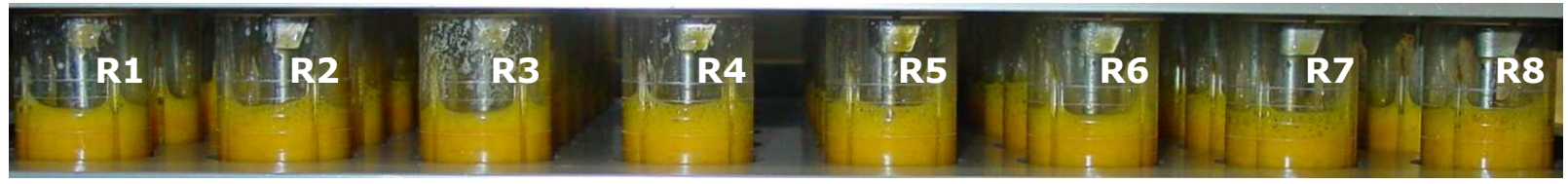

$\mathrm{t}=34.1 \mathrm{~h}$

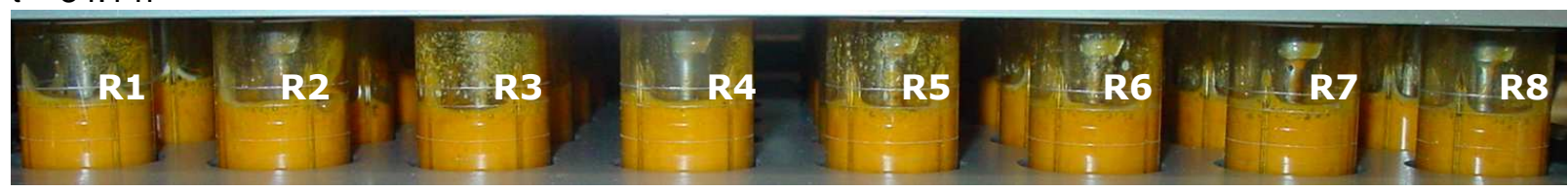

$\mathrm{t}=41.3 \mathrm{~h}$; liquid level in R3, R5, and R6 has collapsed; no bubbles at the surface

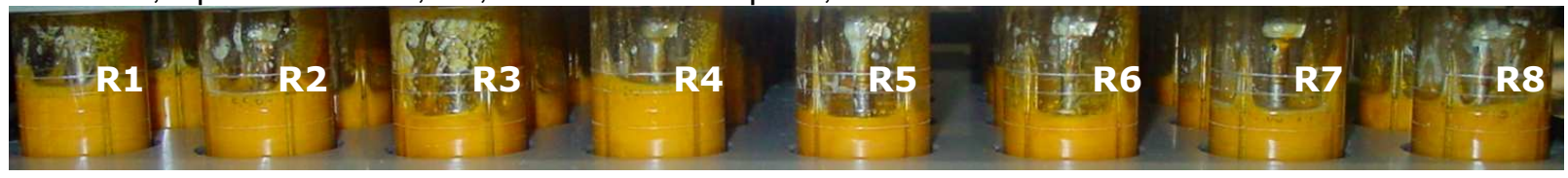

$\mathrm{t}=43,7 \mathrm{~h}$; medium in reactors $\mathrm{R} 3, \mathrm{R} 5$, and $\mathrm{R} 6$ has turned green

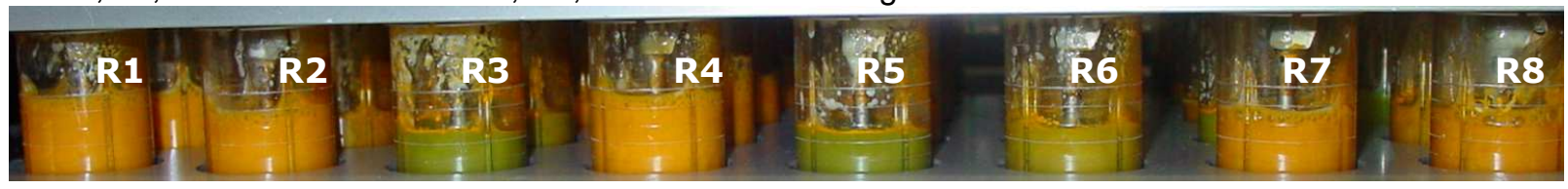

Figure 4-41: Photographs of the first column of reactors (R1-R8) with volumetric scores during a 48fold parallel B2 cultivation at different indicated process times.

In addition to a poor gas input due to resting turbines, a sudden apparent decrease of approximately one milliliter according to the volumetric scores was ob- 
served after 30-40 hours of the processes, which is demonstrated for reactors R3, R5 and R6 in Figure 4-41 by photographs of the first column of reactors during different times of the cultivation. In the described case, hardly any bubbles reached the surface and the medium changed color from orange to green during the next hours and eventually back to orange.

The drop of the liquid level in reactors R3, R5, and R6 between 34 and 41 hours was accompanied by a dramatic descent of the dissolved oxygen in these reactors, as shown in Figure 4-42. During this cultivation a software error lead to a complete process downtime between 38 and 41 hours, during which no feed or base addition and no sampling took place. Only the agitation continued.

Similar collapses of the dissolved oxygen were also observed at other times, as well as during other processes. In addition, gradually decreasing fluctuations in dissolved oxygen were recorded in single reactors, which ultimately also converged to zero (data not shown).

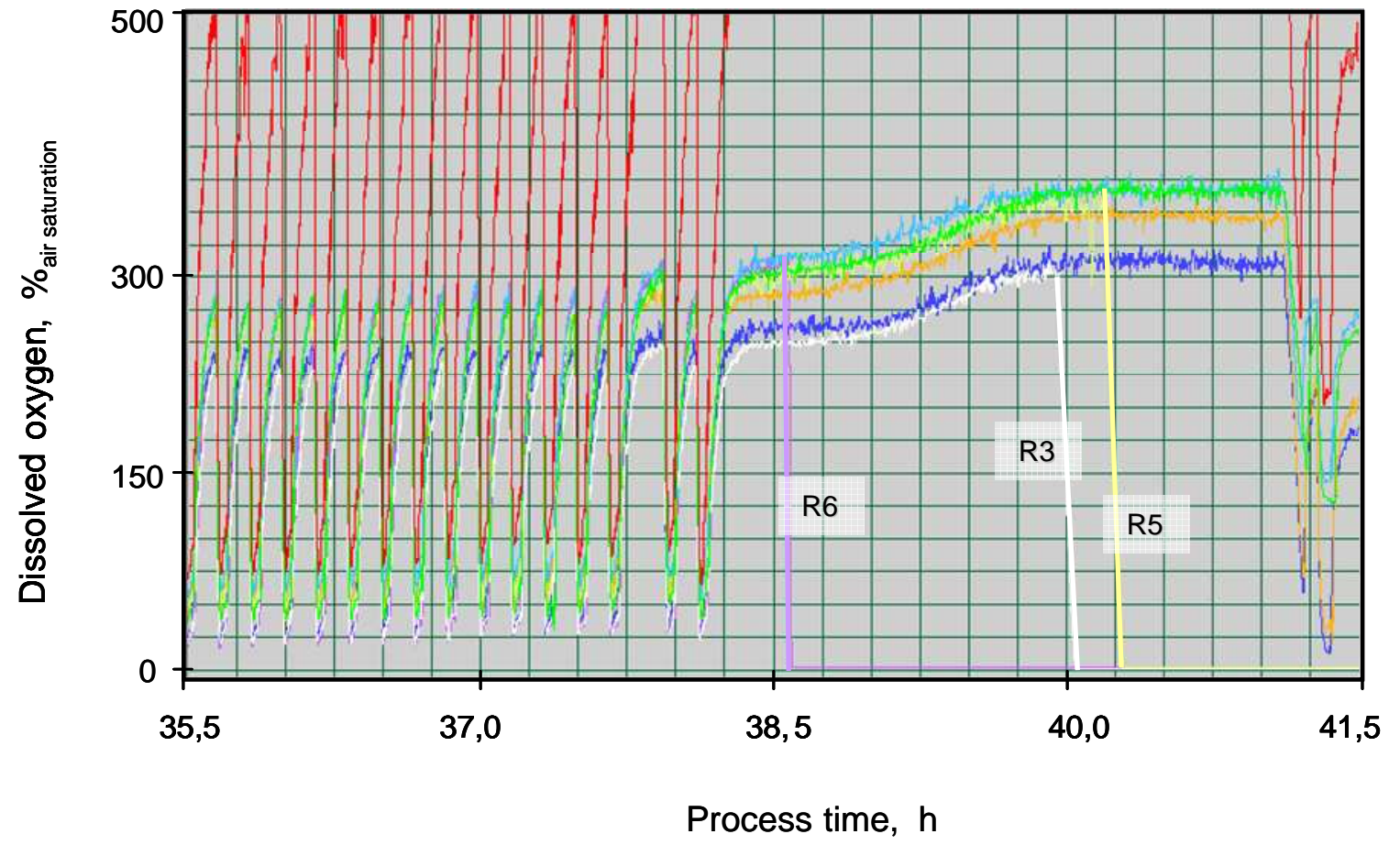

Figure 4-42: Dissolved oxygen in reactors $\mathrm{R} 1-\mathrm{R} 8$ in the first column of the reaction block during six hours after 35.5 hours process time of a 48 -fold parallel B2 cultivation. At approximately 38 hours there was a complete process downtime except for stirring for about three hours. During this time the gas input collapsed for reactor R6 (purple), followed by reactor R3 (white) and R5 (yellow).

Generally the times of the oxygen input failures could be clearly identified via the dissolved oxygen. But the online measurement of dissolved oxygen was only available for one column of the reaction block. Thus the time of possible breaks of the gas input in the other vessels had to be estimated based on the observations 
of the liquid levels, the bubble formation and the color of the cultivation medium. According to this judgment, the reactors of one 48-fold B2 process were categorized depending on the time, at which a proper gas supply seemed to have ceased. The results, presented in Figure 4-43 B, supported data achieved with glucose measurements, in which the accumulation of glucose indicated insufficient gas input in the respective reactors (Figure 4-43 A). The observations revealed that after 40 hours more than $30 \%$ and at the end $(t=53 \mathrm{~h}$ ) more than $65 \%$ of the reactors suffered from gas input failures.

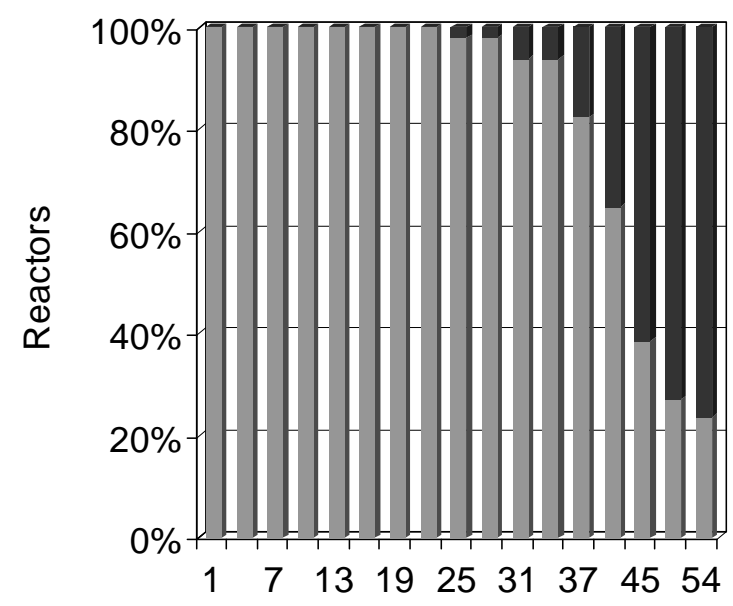

A

Process time, $\mathrm{h}$

reactors with glucose accumulation reactors without glucose accumulation

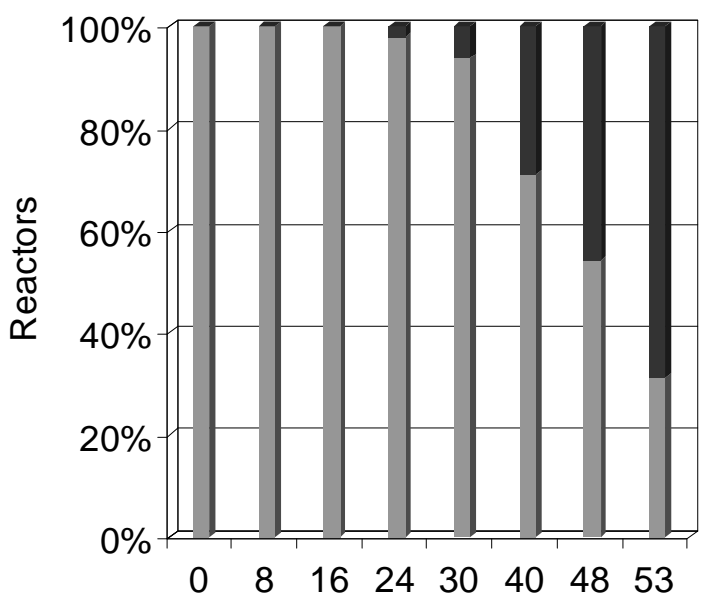

B

Process time, $\mathrm{h}$

reactors with gas input failure reactors with proper gas input

Figure 4-43: Relative quantity of 48 reactors with gas input failures after increasing process time during a 48-fold parallel B2 cultivation in the milliliter setup, estimated based on the detection of glucose accumulation $(A)$ or on observations regarding the liquid level, the bubble formation and the color of the reaction medium (B).

The hollow axles of the reactors with the described phenomena were found to be stuck with riboflavin crystals and cells, when the setup was taken apart after completed cultivations.

\section{Discussion}

The results presented in this subsection showed that the online monitoring of the dissolved oxygen was an essential tool for controlling the B2 processes in the milliliter setup and for understanding the resulting performances in individual reactors depending on the respective oxygen supply. The employed sensor unit was a prototype as well as the oxygen-sensitive fluospots inside the reactors in most of the experiments. It was therefore not surprising that some of the dissolved oxy- 
gen values, particularly in the ranges $>100 \%$ air saturation, were outside the theoretically expected values or showed a drift over elevated process time.

The phenomenon of collapsing liquid levels accompanied by the absence of bubbles at the surface of the respective reactors occurred despite running stirrers. The most likely explanation for the resulting failure of oxygen supply was that the hollow axles got stuck by particles at some point during the cultivation, which no longer allowed the passage of gas into the medium. The times reported for gas input failures supported the detection of glucose buildup as a result of the insufficient oxygen input into the respective reactors.

A sufficient oxygen supply is one of the most crucial parameters for successful B2 cultivations with respect to the amount of riboflavin that has to be produced. It is therefore of utmost importance to modify the gas supply strategy, for example by widening the inner diameter of the hollow axles in the milliliter setup.

\subsubsection{Volumetric increase and evaporation in the milliliter setup}

For a dependable comparison of concentrations in the milliliter vessels with those in the stirred tank reactors at laboratory scale, the balance between liquid additions and evaporation had to be the same in both systems in order to yield a similar volumetric increase throughout the whole process.

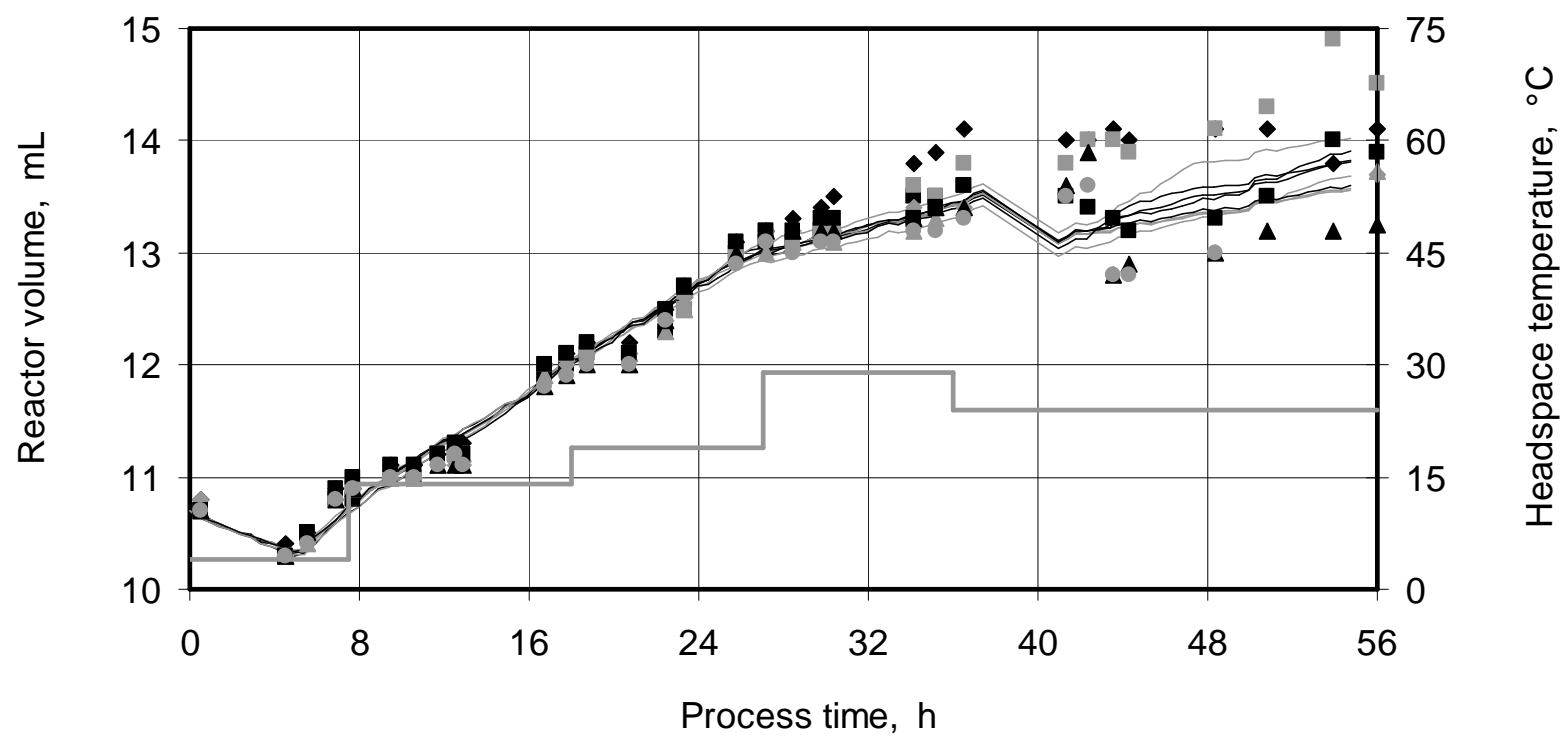

Figure 4-44: Volumetric increase in eight parallel reactors in the first column of the reaction block during a 48-fold B2 cultivation. Evaporation was controlled by headspace cooling at different temperatures, indicated by the bold grey line. Thin lines are the theoretical volumes based on summarizing all added and withdrawn aliquots and on estimated evaporation rates, which were $60,110,130,180$ or $160 \mu \mathrm{L} \mathrm{h}^{-1}$ for headspace cooling temperatures 4 , $14,19,29$ or $24^{\circ} \mathrm{C}$, respectively. Symbols represe nt the volumes in the same reactors recorded according to external scores on the reactor walls. Final volumes are based on gravimetric endpoint determinations. Feeding was started after $5.25 \mathrm{~h}$ and was interrupted accidentally for three hours after 38 hours. 
For an estimation of actual reactor volumes during B2 processes in the milliliter bioreactors, volumetric scores were incised into the reactor walls, visible in the photographs of Figure 4-41, for known volumes under representative conditions regarding stirrer speed and gas input. Thereby the volumes could be estimated via the liquid level in eight parallel reactors in the first column of the reaction block, presented as grey and black symbols in Figure 4-44 exemplary for one cultivation. The approximate error on volumes recorded according to this method was $10 \%$. The theoretical volumes, which resulted from balancing the input (feed, base, antifoam) and output volumes (evaporation and sampling for $\mathrm{pH}$ and at-line monitoring), were matched to the recorded volumes by estimating appropriate evaporation rates for each headspace cooling temperature. The volumetric profiles obtained with this method are displayed as lines in Figure 4-44.

According to these estimations the evaporation rates in reactors with $10 \mathrm{~mL}$ initial volume, which were stirred at $2700 \mathrm{rpm}$ and $39^{\circ} \mathrm{C}$, were between $40 \mu \mathrm{L} \mathrm{h}^{-1}$ and $200 \mu \mathrm{L} \mathrm{h}^{-1}$ per reactor depending on the degree of headspace cooling and possibly on the actual reactor volume. The correlation between evaporation rates and the temperature used for headspace cooling is shown in Figure 4-45. It allowed adjusting the desired evaporation rates during the B2 processes by applying linear regression (slope $=4.85 \mu \mathrm{L} \mathrm{h}^{-1}{ }^{\circ} \mathrm{C}^{-1}$, offset $=40.2 \mu \mathrm{L} \mathrm{h}^{-1}$ ).

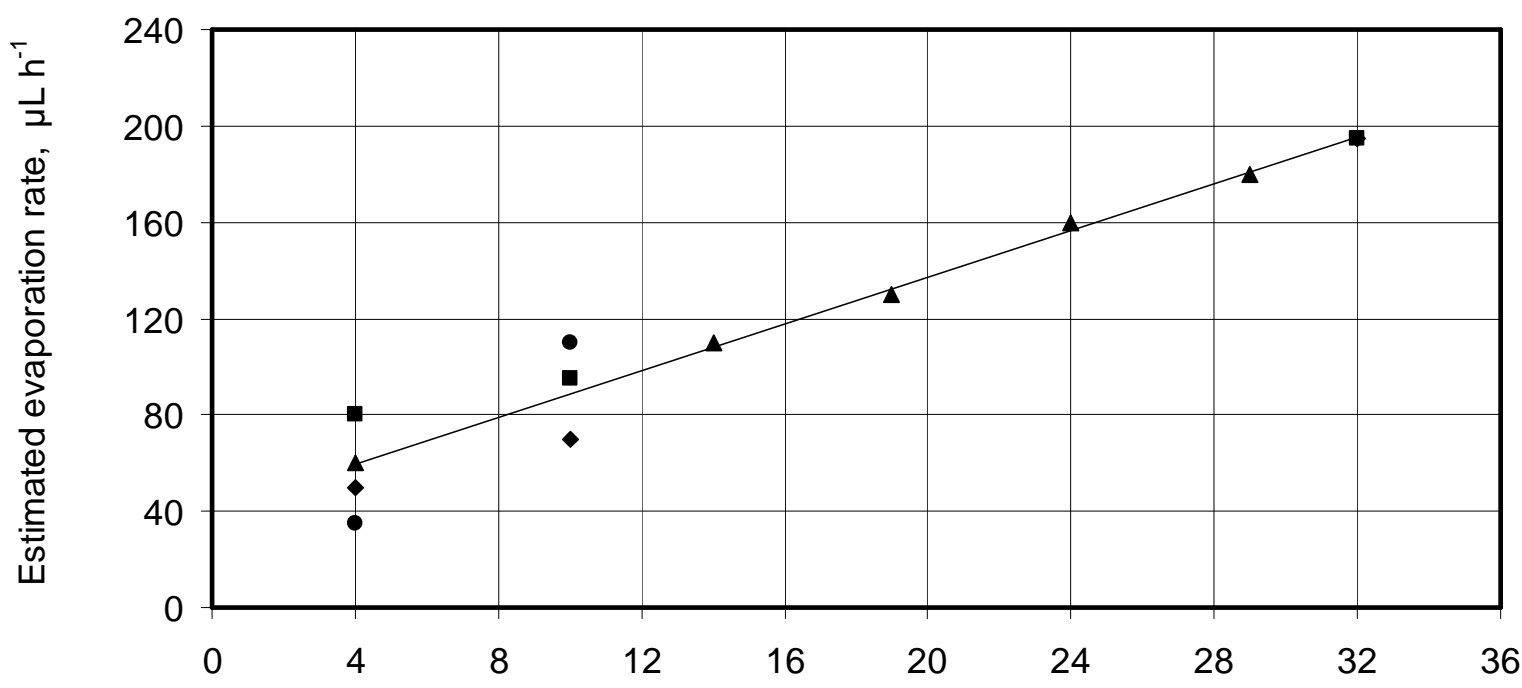

Temperature for head space cooling, ${ }^{\circ} \mathrm{C}$

Figure 4-45: Evaporation rates from milliliter bioreactors with $10-14 \mathrm{~mL}$ volume, operated at $39^{\circ} \mathrm{C}$, as a function of the temperature used for headspace cooling. $32{ }^{\circ} \mathrm{C}$ was the estimated temperature for no cooling. The evaporation rates were estimated during four independent parallel B2 cultivations based on the best fit between the theoretical reactor volumes that were calculated using these rates and the volumes recorded according to external volumetric markings on reactors of the first column in the reaction block. 
The aim of the volumetric adjustment during the B2 processes in the milliliter setup was to yield an increase in liquid volume comparable to that at the reference scale. The setup at laboratory scale was equipped with a very efficient offgas cooler and hence the water loss was insignificant.
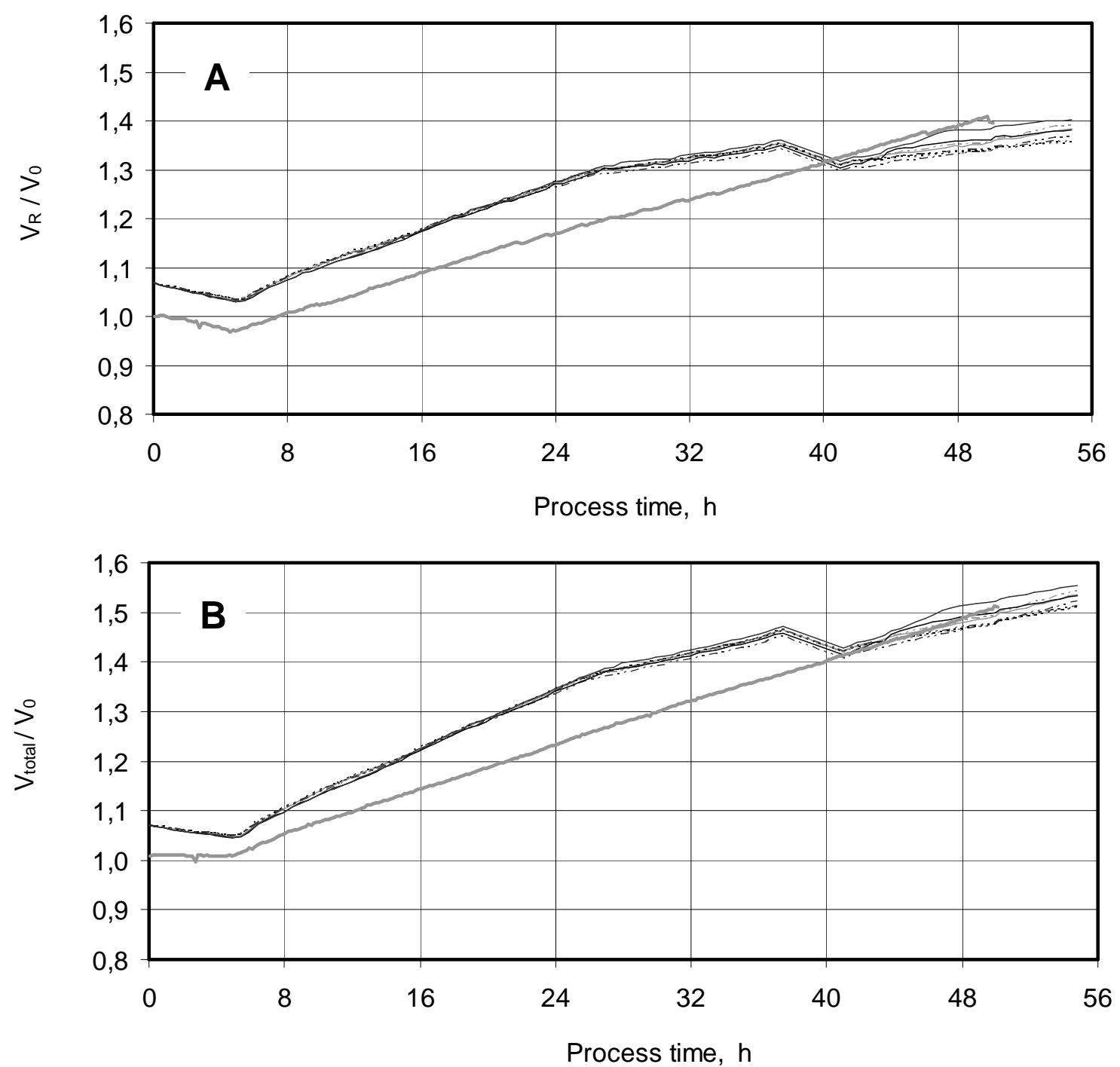

Figure 4-46: Normalized volumetric increase during B2 processes in eight parallel milliliter bioreactors (thin lines) compared with the reference at laboratory scale (bold grey line). The initial volume $V_{0}$ at laboratory scale was $3 \mathrm{~L}$, the actual volume $V_{R}$ was based on the reactor weight. The initial volume during the cultivation at milliliter scale was $10.7 \mathrm{~mL}$, but feed addition was for $V_{0}=10.0 \mathrm{~mL}$, which was used as $V_{0}$ for normalizing. The liquid handling system stopped accidentally after 38 hours for three hours so that no additions took place during this time. A, modeled reactor volume $V_{R}$ according to the volumetric balance. $B$, total volume $V_{\text {total }}$ inclusive of the accumulated withdrawn sample volumes.

The high evaporation occurring in the miniature bioreactors had to be compensated by diluting the additive solutions (feed and base) 1:2 with respect to the original protocol. This was advantageous also for the handling of these solutions with the laboratory robot. The volumetric profiles for cultivations at both scales 
are displayed in Figure 4-46, normalized by dividing the reactor volumes by the respective initial volume. In the milliliter reactors the actual initial volumes were $10.7 \mathrm{~mL}$ instead of the intended $10.0 \mathrm{~mL}$ due an inaccurate calibration of the dispenser used for filling the reactors. The amount of feed addition was kept according to an initial volume of $10.0 \mathrm{~mL}$, which was therefore used for normalizing the values. This is the reason why the volumetric courses for the milliliter scale start above the reference.

The rise of the reactor volumes with increasing process time, which is approximately parallel to the course of the reference process, demonstrates a similar volumetric increase at either scale. The volumes in the milliliter vessels remained approximately 5-8 \% higher compared to the reference until an unintended process downtime after 38 hours process time, which resulted in a reduction of the volume so that the final volumes were about the same for either scale.

The increases of the actual reactor volumes or of the total reactor volume inclusive of the withdrawn sample volumes were different at each scale, because the relative amount of total sample volume was larger at the milliliter scale due to the frequent sampling for the at-line $\mathrm{pH}$ measurement.

\section{Discussion}

The volumetric scores did only allow an estimation of the actual volumes. The method was complicated by the eventually instable liquid levels due to the collapsing gas input and was thus also dependant on the judgment of the respective operator recording the values. But generally these data were very valuable for monitoring the process performance and for an approximation of the evaporation.

The similarity of the volumetric increase during the cultivations despite different initial volumes supported the equivalence of the process operation at either scale. Since the reactor volumes in the milliliter vessels ended up in the same range with the respective values at laboratory scale after 38 hours and were only 5-8\% higher before that, the comparison of concentrations at either scale was possible.

Generally the normalized volumetric profiles confirmed that the evaporation from the milliliter vessels, which was significantly higher than from the stirred tank reactor at laboratory scale, could be compensated by diluting the additive solutions feed, base and antifoam. The profiles could be further adjusted by changing the temperature for cooling the headspace of the milliliter reactors to meet the volumetric profile of the reference process at laboratory scale. 


\subsubsection{Growth and productivity at the milliliter scale}

This subsection provides the most important outcome of this thesis regarding the performance of eightfold and 48-fold parallel B2 processes in the milliliter system. Glucose consumption, growth and the generation of riboflavin by $B$. subtilis during these processes are addressed, as well as the reliability of the analytical methods used to assess these quantities. The occuring symbols will each be representative of one particular parallel cultivation, using triangles or squares for two 48-fold processes and diamonds, circles or crosses for three eightfold processes.

\section{Glucose consumption}
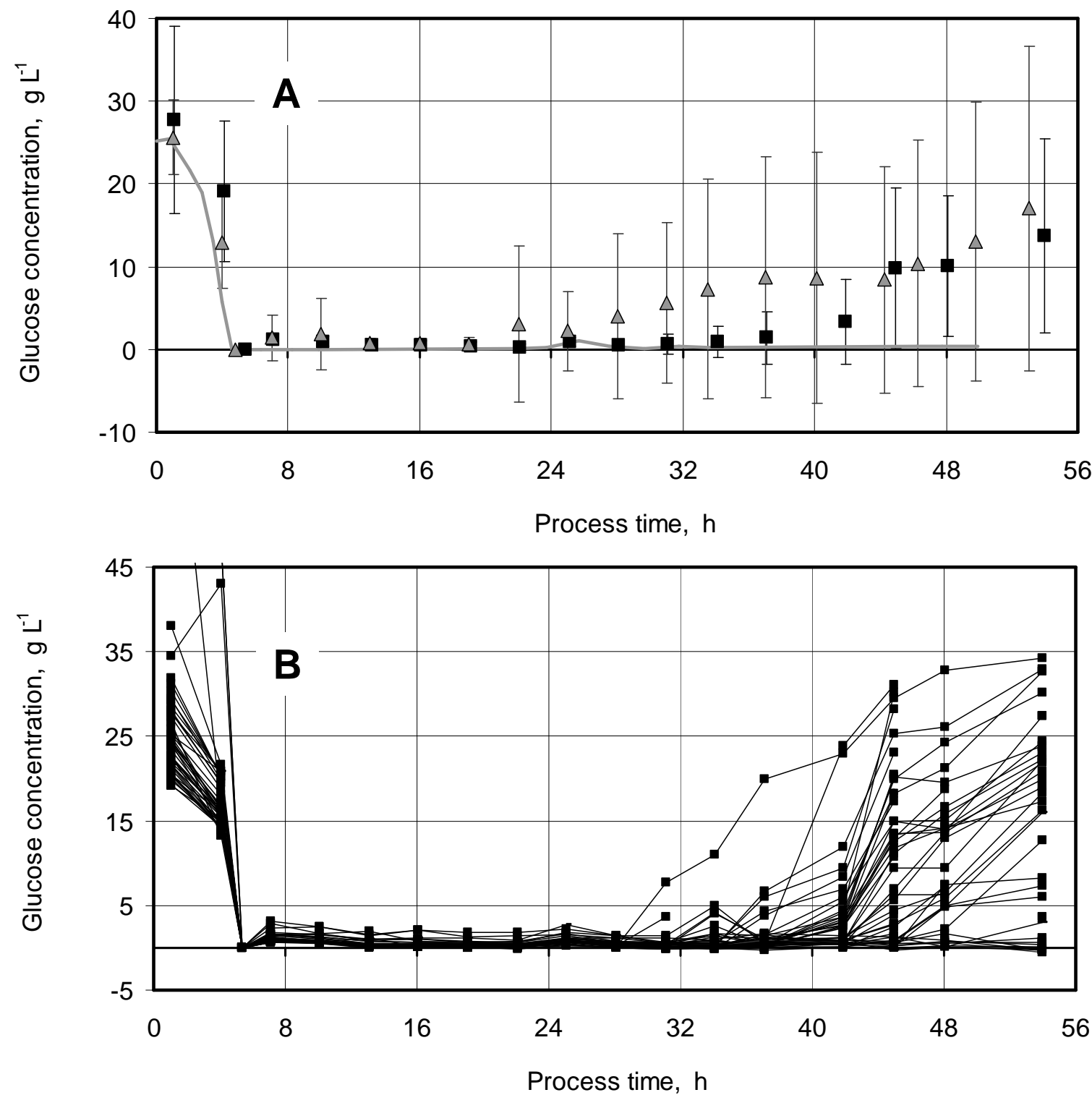

Figure 4-47: A, Average glucose concentrations during two 48-fold parallel B2 processes estimated at-line represented as black squares or grey triangles, compared with the intermittent reference process at laboratory scale (grey line). Error bars are based on the standard deviation. B, glucose concentration in the individual vessels of the process represented with squares in part A. Symbols are connected with straight lines for a better display. 
The glucose consumption during the first 24 hours of parallel B2 processes in the milliliter setup was essentially the same as during the reference processes at laboratory scale (Figure 4-47 ). The batch phases lasted 4-6 hours. After 20 hours or 35 hours during either of the displayed 48-fold parallel B2 processes, respectively, substrate started accumulating in certain reactors, which is displayed individually for the reactors of one of the processes in Figure 4-47 B. In some experiments the final at-line values were confirmed with offline analysis of the medium (data not shown). The glucose accumulation resulted in significantly reduced parallel reproducibility, expressed as error bars based on the standard deviation.

\section{Growth}

The growth of $B$. subtilis during two 48-fold parallel B2 cultivations was equivalent to the intermittent reference process at laboratory scale during the first 24 hours of the processes with standard deviations between the parallel reactors $<6 \%$, as shown in Figure 4-48, in which also the continuously fed reference is displayed. The biomass concentrations after 24 hours were clearly lower in the milliliter system than during the continuously fed cultivation, but only slightly lower than during the intermittently operated process at laboratory scale.

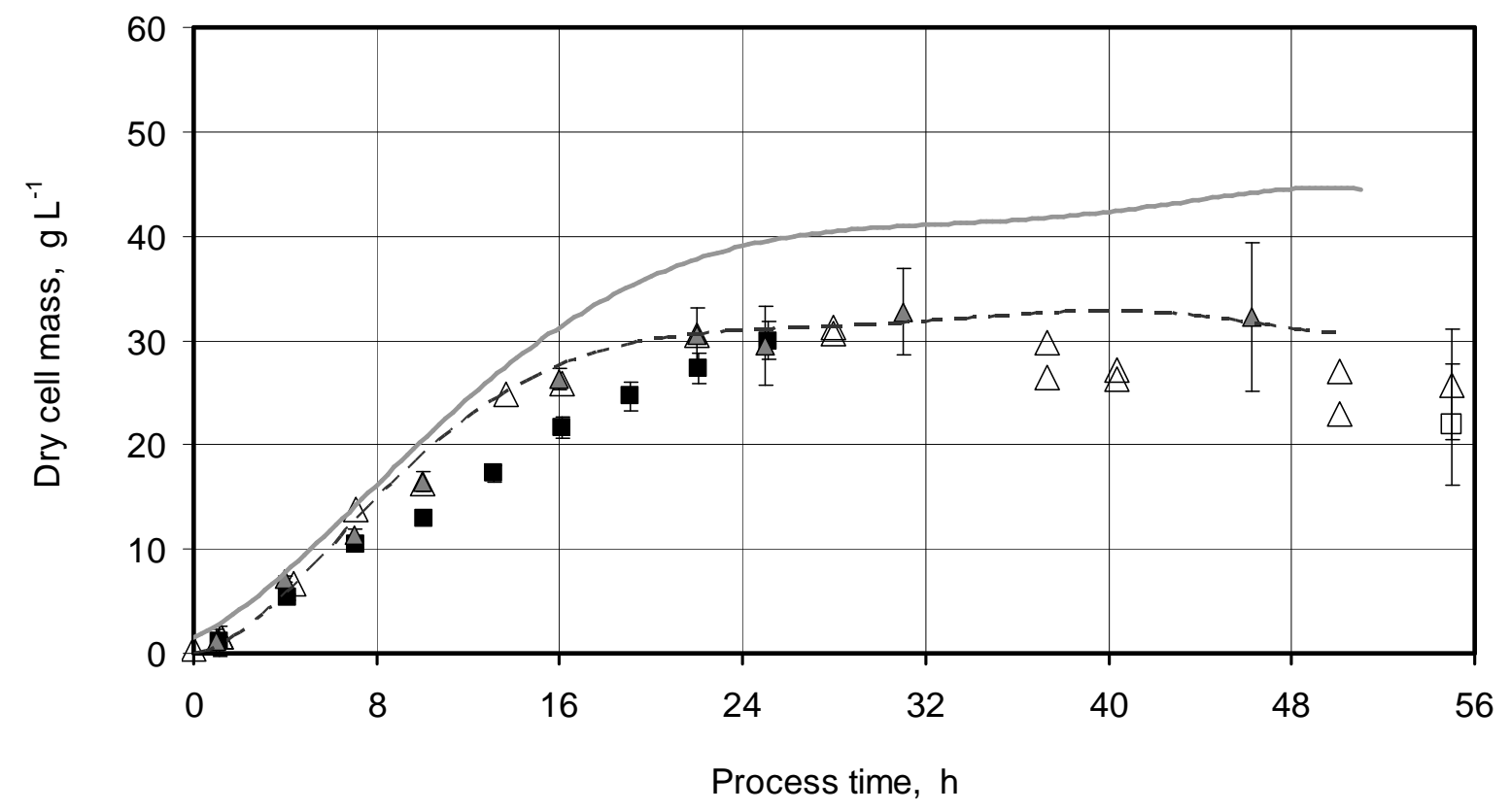

Figure 4-48: Dry cell mass during two 48-fold B2 cultivations (symbols) compared with the intermittent reference process at laboratory scale (dashed line) and the continuously fed reference (solid grey line). Black and grey symbols are based on the averages of the at-line determined optical density for all running reactors. Open symbols stand for gravimetrically measured offline data of single reactors during one of the 48-fold cultivations. The final values at 55 hours are the averages of gravimetrically determined values of the parallel reactors after completion of the respective cultivation. Error bars represent standard deviations. 
The black and grey symbols in Figure 4-48 are based on averages of the at-line determinations during the milliliter processes via optical density. A major problem for this at-line monitoring was that after approximately 24 hours the automatic adjustment of the light ducts for absorbance measurements in the photometric plate reader did not function correctly anymore. This resulted in falsely high optical densities which are thus not displayed in the figure.

During one of the portrayed processes offline samples were taken from single reactors for the gravimetric assessment of the dry cell mass. The results are shown as open symbols in the same figure, exposing a decrease of dry cell mass during the second half of the process to final values of $26 \mathrm{~g} \mathrm{~L}^{-1} \pm 20 \%$, similar to those of the other process with $22 \mathrm{~g} \mathrm{~L}^{-1} \pm 20 \%$, during which the liquid handling was accidentally interrupted for approximately three hours after 38 hours.

In an eightfold parallel experiment, the average of gravimetrically determined dry cell masses was $36.2 \mathrm{~g} \mathrm{~L}^{-1} \pm 11 \%$ for seven reactors in an average final volume of $12.4 \mathrm{~mL} \pm 3 \%$ before glucose accumulation was detected (data not shown). The corresponding volumetric increase of 1.24 during this experiment compared to 1.4 during the reference process indicated that the evaporation during this cultivation was not sufficiently compensated. If the volumetric increase of the reference was used for an estimation of the appropriate dry cell mass in a corrected final volume for this experiment, the respectively more diluted final dry cell mass would have been $31.9 \mathrm{~g} \mathrm{~L}^{-1} \pm 11 \%$. This is in the range of the intermittently fed process at laboratory scale.

\section{Riboflavin production}

The riboflavin productivity of Bacillus subtilis RB50::[pRF69] $]_{\mathrm{n}}$ decreased after several passages of the original working cell bank, yielding only $9-11 \mathrm{~g} \mathrm{~L}^{-1}$ riboflavin concentration after 48 hours (Figure 4-50) compared to $13-14 \mathrm{~g} \mathrm{~L}^{-1}$ previously. The integrated product yields for two 48 -fold B2 processes in the milliliter setup were in the range of $0.030-0.045 \mathrm{~g} \mathrm{~g}^{-1}$ after $48-53$ hours (Figure 4-49). During one process, represented with squares in this figure, the liquid handling was accidentally interrupted for approximately three hours after 38 hours, which resulted in a reduced overall yield compared to the other processes. The error bars in this figure are based on the average riboflavin concentrations without clear outliers. They revealed that the riboflavin values derived from the automated at-line quantification were afflicted with variations up to $20 \%$. Nevertheless, the average riboflavin concentrations matched the offline values of single reactors measured via absorbance. This is demonstrated in Figure 4-50 for three different parallel B2 cultivations at the milliliter scale. 


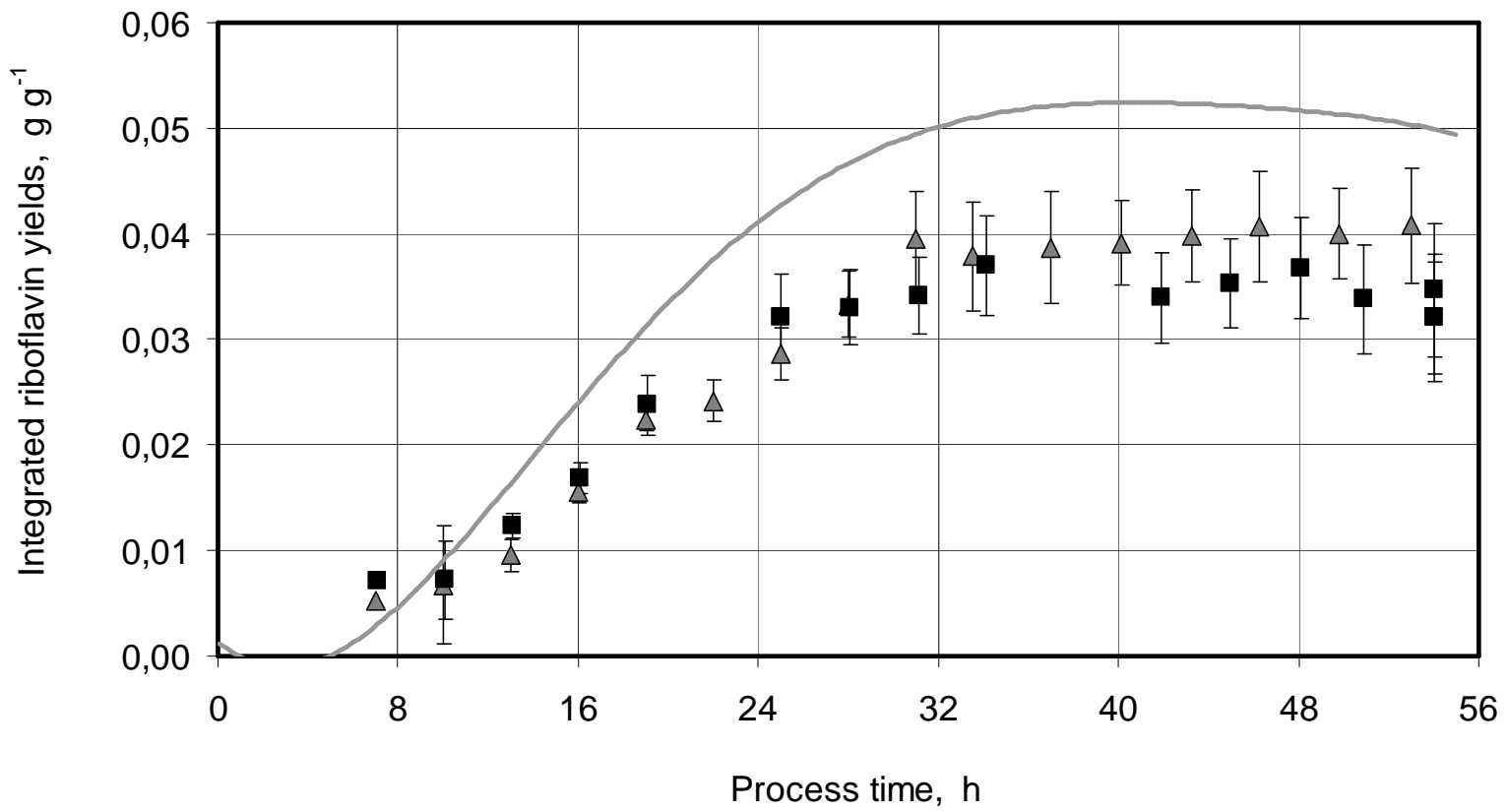

Figure 4-49: Integrated riboflavin yields for two 48-fold parallel B2 processes in the milliliter setup (symbols) compared with the continuously fed reference process at laboratory scale with the original $B$. subtilis strain (grey line). Error bars are based on the standard deviation of the at-line determined riboflavin concentrations without clear outliers.

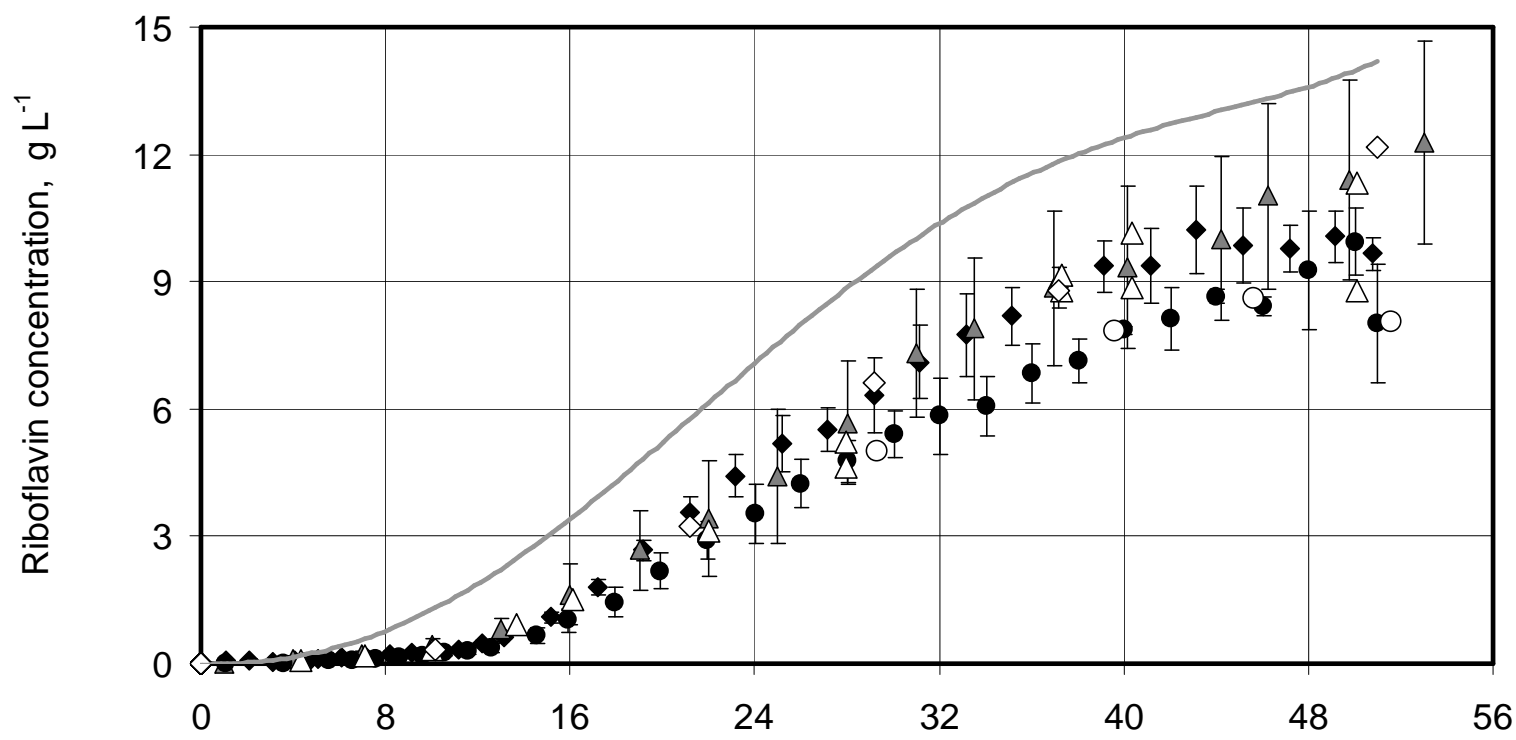

Process time, $\mathrm{h}$

Figure 4-50: Riboflavin concentrations of one 48-fold and two eightfold parallel B2 processes in the milliliter setup (triangles and circles or diamonds, respectively), determined at-line via fluorescence (black and grey symbols) or offline via absorbance (white symbols). The atline values and error bars are based on averages without clear outliers. The offline values were taken from different single reactors. The grey line represents the continuously fed reference process at laboratory scale with the original $B$. subtilis strain. 


\section{Discussion}

The at-line analysis for glucose detection was very well suited for the monitoring of glucose accumulations in individual reactors. The buildup of substrate generally indicated insufficient oxygen supply for the desired metabolism and could clearly be attributed to improper gas input into the respective milliliter vessel. Under appropriate conditions during the feeding phase glucose was only detected below the lower detection limit.

The automated assessment of glucose in the milliliter setup should be regarded as a valuable qualitative indicator rather than a precise tool for quantifying exact glucose concentrations because of the considerable variations between the determinations for parallel reactors, most likely due to the limited pipetting accuracy of the laboratory robot.

Growth of $B$. subtilis during parallel B2 processes in the milliliter setup was equivalent to that of the intermittent reference process at laboratory scale, particularly during the first 24 hours. The reduced biomass concentrations during intermittently operated B2 processes compared to continuously fed processes could most likely be attributed to the elevated dissolved oxygen tension whenever the glucose was consumed. The slightly higher maximum oxygen input at the milliliter scale (55-60\% in the gas flow compared to $50 \%$ at laboratory scale) may have further increased the oxygenic stress to the cells. Since the overall riboflavin generation was not affected, the producing fraction of the biomass was expected to be the same for either operational mode in each of the different setups.

The lower values of gravimetrically determined dry cell masses during the second half and at the end of the two processes at the milliliter scale (Figure 4-48) could be attributed to the inconsistent gas input in numerous reactors after 30 to 40 hours. In addition, an accidental interruption of the liquid handling during one of these processes kept the cells from receiving feed or base for approximately three hours after 38 hours. This certainly had a further negative impact on the dry cell mass. The gravimetrically determined dry cell masses at the end of the eightfold parallel experiment, during which no glucose accumulation occurred, indicated that equal growth in the milliliter vessels compared to the stirred tank reactors at laboratory scale could also be obtained during the second half of a B2 process, given that sufficient oxygen and substrates were supplied.

The reduced riboflavin productivity of the finally used $B$. subtilis strain compared to the original Bacillus subtilis RB50::[pRF69] n may have been caused by a loss of chromosomal sequences containing copies of the rib operon which originated from plasmid pRF69. This is assumed, even though each of the four passages of the cells over the course of two years had been carried out in the presence of 
$60 \mathrm{mg} \mathrm{L}^{-1}$ chloramphenicol, which should prohibit this loss (Young, 1984). The lower product generation was not only observed during parallel processes in the milliliter system, but also during two continuously fed reference processes at DSM NP when the same strain was used. Figure 4-51 depicts these processes as alternative references for the performance of the B2 process in the milliliter setup.

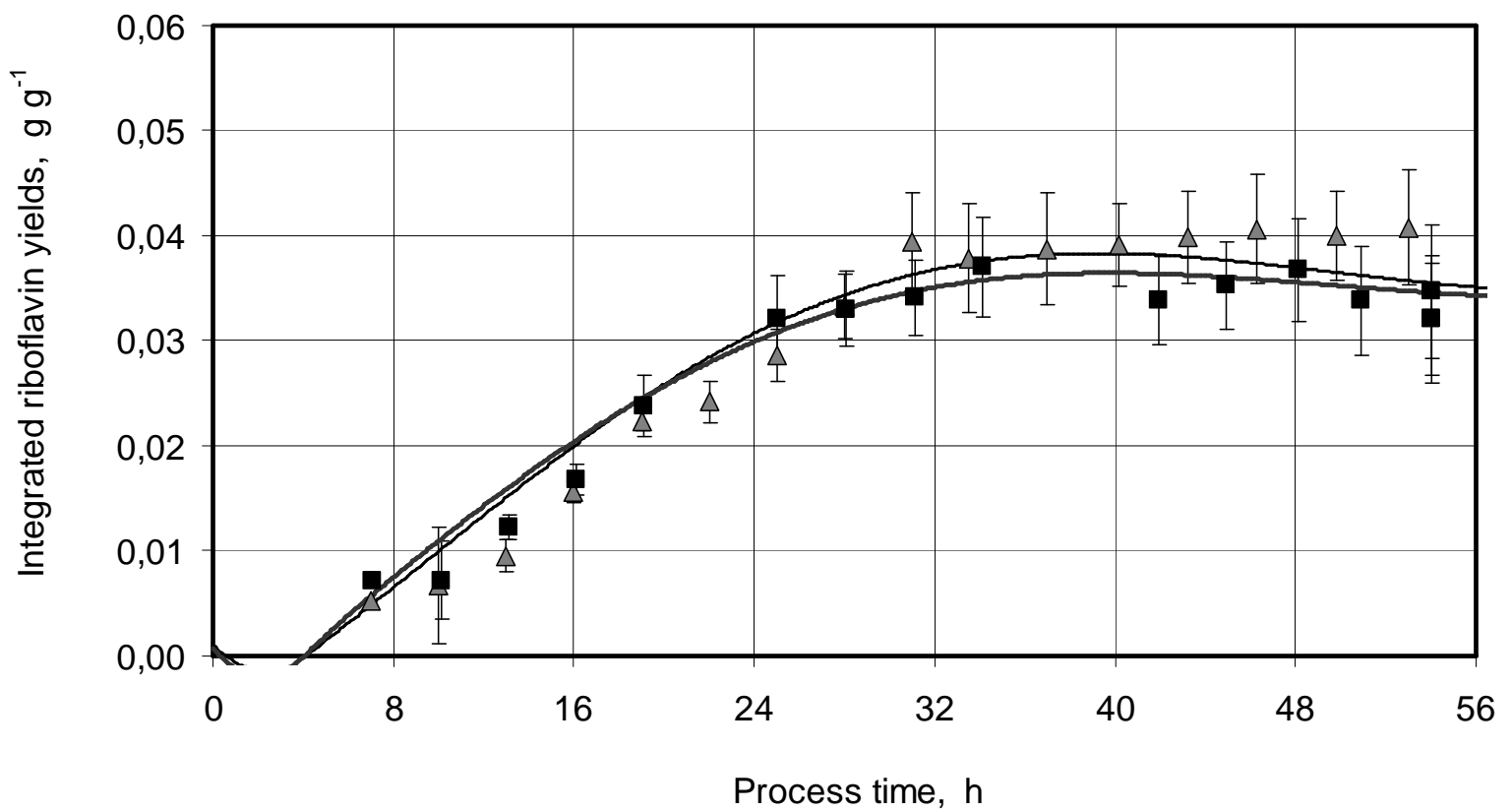

Figure 4-51: Integrated riboflavin yields for two 48-fold parallel B2 processes in the milliliter setup (symbols) compared with two continuously fed reference processes at laboratory scale at DSM NP with the same bacterial strain (lines). Error bars are based on the standard deviation of the at-line determined riboflavin concentrations without clear outliers.

The data in Figure 4-51 show that the riboflavin yields generated by $B$. subtilis in the milliliter setup corresponded to those during the reference process at DSM NP with the same strain. This is the most important result of the experiments. It allows considering the setup suitable for the screening of different $B$. subtilis strains or process strategies for improved B2 production.

However, the variations and deviations, with which the automated at-line quantification of riboflavin was afflicted, especially towards the end of each process, were too high for a reliable characterization of the process performance. The reason for the low precision of the automated method was most likely the limited accuracy of the pipetting robot during the applied dilution and mixing steps. Despite the large variations, the at-line analysis was very valuable for monitoring the processes, especially when the resulting values were examined critically. The procedure could potentially be improved by a simple reconditioning of the robotic liquid handling system. But for a reliable quantification of riboflavin concentration, offline determinations with HPLC should be carried out at least every 24 hours. 


\subsubsection{Sequential and parallel reproducibility}

In Figure 4-52 the riboflavin concentrations during four independent parallel cultivations with either eight or 48 vessels are displayed in order to demonstrate the sequential reproducibility of the performance of B2 processes in the milliliter setup.

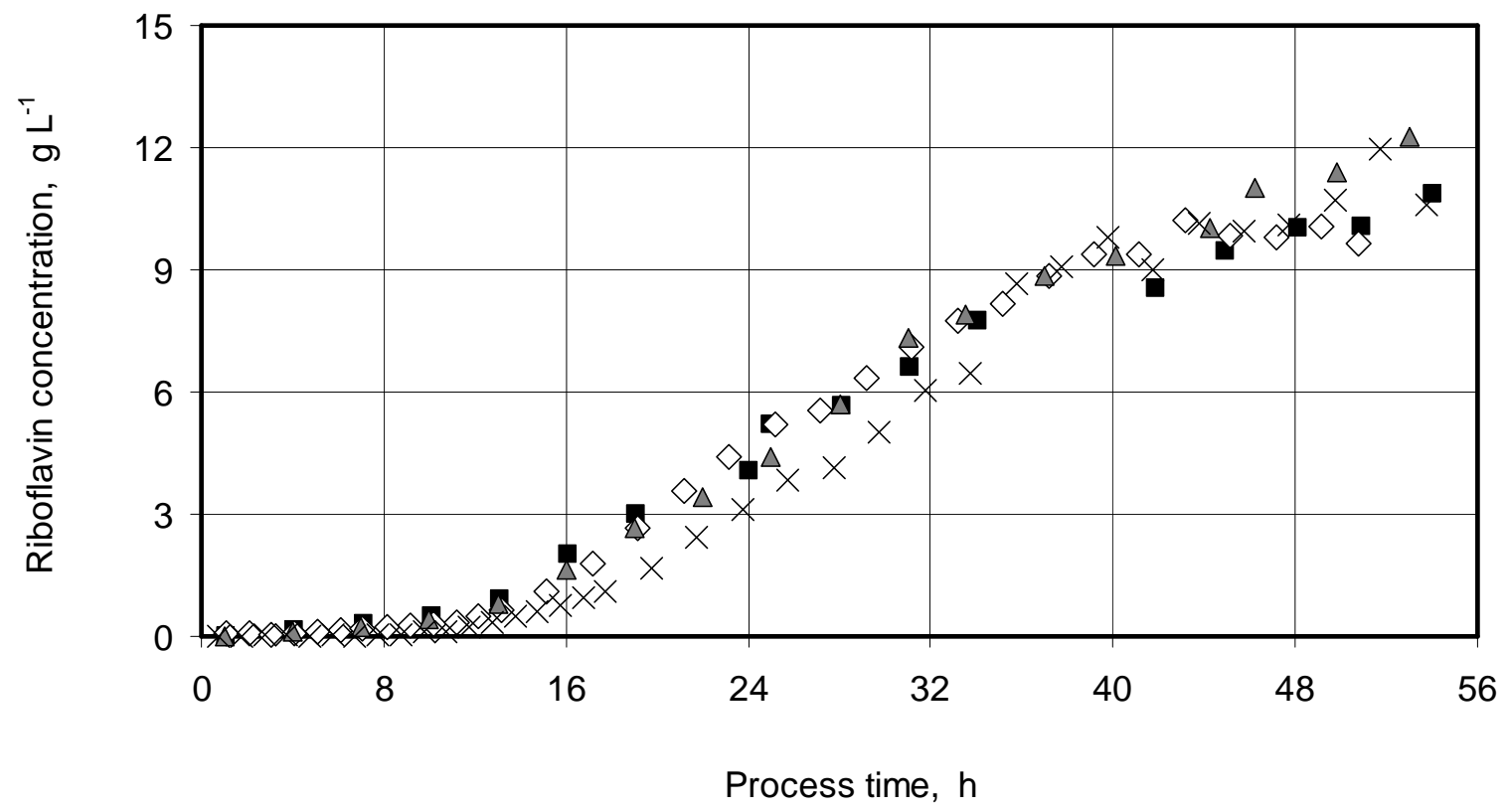

Figure 4-52: Sequential reproducibility regarding the generated riboflavin concentrations during two 48-fold and two eightfold parallel B2 processes in the milliliter setup (dark symbols and white diamonds or crosses, respectively). The values are based on the averages of the at-line data of properly running reactors without clear analytical outliers.

The resulting profiles were very similar, and in all of the processes the final riboflavin concentration reached $10-12 \mathrm{~g} \mathrm{~L}^{-1}$. Only the curve for the eightfold parallel process represented with crosses was lower until 32 hours. The volume between 16 and 32 hours during this cultivation was higher compared to the other processes (data not shown).

The sequential and parallel reproducibility of the growth behavior of $B$. subtilis during the first 24 hours of numerous B2 processes in the milliliter setup was excellent, with variations between parallel reactors $<6 \%$ according to the at-line determined data based on optical density (data not shown). However, whenever the gas input was not sufficient or feeding was interrupted over extended periods of time, the dry cell mass decreased in the respective reactors (data not shown).

Regarding the demonstration of parallel reproducibility of the performance of B2 cultivations in the milliliter setup, the amount of available data was limited. This was because in most of the parallel B2 processes during this thesis, some of the operational parameters were optimized by applying different strategies to individ- 
ual reactors, for example the antifoam addition or aspects of the automation. As a consequence, the results between single reactors of parallel cultivations differed. Only during one 48-fold experiment (represented with squares in the previous subsection) all the vessels were treated the same in order to assess the parallel reproducibility. The results of this experiment are displayed in Figure 4-53 in schemes of the reaction block with individual quantities for each properly running reactor. Additionally, the averages for each column or row of the reaction block are given in order to identify local predispositions.

Final $c_{X}=T D M-c_{P}, \mathrm{~g} \mathrm{~L}^{-1}$

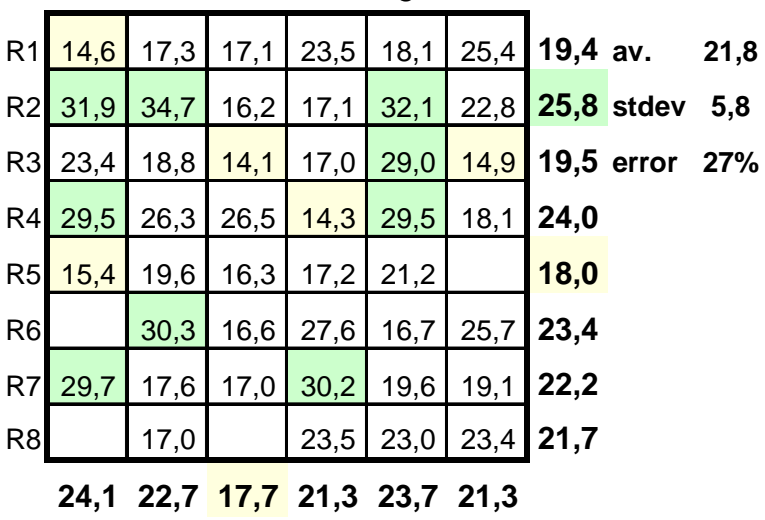

Final volume $V_{R}, \mathrm{~mL}$

\begin{tabular}{|c|c|c|c|c|c|c|c|}
\hline R1 & 14,1 & 14,4 & 13,7 & 13,5 & 14,1 & 14,5 & 14,1 av. \\
\hline $\mathrm{R} 2$ & 13,9 & 13,8 & 14,2 & 13,8 & 13,3 & 15,1 & 14,0 stdev \\
\hline $\mathrm{R} 3$ & 13,7 & 13,3 & 13,4 & 13,1 & 13,0 & 13,4 & 13,3 error \\
\hline R4 & 14,5 & 13,8 & 13,3 & 13,5 & 13,0 & 14,4 & 13,8 \\
\hline R5 & 13,7 & 13,2 & 12,9 & 12,8 & 13,0 & 13,2 & 13,1 \\
\hline R6 & 12,9 & 13,7 & 13,3 & 13,2 & 13,2 & 13,9 & 13,4 \\
\hline R7 & 13,2 & 13,0 & 12,5 & 12,7 & 12,4 & 13,3 & 12,9 \\
\hline $\mathrm{R} 8$ & 13,0 & 13,4 & & 13,2 & 12,5 & 13,6 & 13,1 \\
\hline
\end{tabular}

Final $c_{P}, \mathrm{~g} \mathrm{~L}^{-1}$

\begin{tabular}{|c|c|c|c|c|c|c|c|c|}
\hline R1 & 8,4 & 8,6 & 9,1 & 8,8 & 8,0 & 9,9 & 8,8 av. & 9,1 \\
\hline R2 & 9,8 & 8,2 & 8,3 & 8,7 & 8,3 & 8,7 & 8,6 stdev & v 0,6 \\
\hline R3 & 8,5 & 8,5 & 8,7 & 8,9 & 9,6 & 9,2 & 8,9 error & $7 \%$ \\
\hline R4 & 9,7 & 8,7 & 8,9 & 10,1 & 9,9 & 9,7 & 9,5 & \\
\hline R5 & 8,5 & 5,8 & 9,4 & 10,1 & 6,3 & 10,0 & 9,5 & \\
\hline R6 & 9,4 & 9,3 & 8,6 & 9,4 & 8,6 & 9,2 & 9,1 & \\
\hline R7 & 9,5 & 9,3 & 9,1 & 8,5 & 9,7 & 9,5 & 9,3 & \\
\hline R8 & 9,2 & 9,0 & & 8,4 & 8,6 & 10,2 & 9,1 & \\
\hline & 9,1 & 8,8 & 8,9 & 9,1 & 8,9 & 9,6 & & \\
\hline
\end{tabular}

Final $c_{P} \cdot V_{R}, \mathrm{mg}$

\begin{tabular}{|c|c|c|c|c|c|c|c|}
\hline & 118 & 124 & 124 & 120 & 112 & 144 & 124 av. \\
\hline & 137 & 113 & 118 & 120 & 110 & 131 & 121 stdev \\
\hline & 117 & 114 & 116 & 116 & 125 & 123 & 118 error $7 \%$ \\
\hline R4 & 140 & 120 & 118 & 136 & 129 & 140 & 131 \\
\hline & 116 & & 121 & 128 & & 132 & 124 \\
\hline & 121 & 127 & 114 & 125 & 113 & 128 & 121 \\
\hline 1 & 126 & 121 & 114 & 108 & 121 & 126 & 119 \\
\hline & 120 & 120 & & 110 & 107 & 138 & 119 \\
\hline & 124 & 120 & 118 & 120 & 117 & 133 & \\
\hline
\end{tabular}

Figure 4-53: Final results of a 48-fold parallel B2 cultivation in the milliliter setup after $53 \mathrm{~h}$. Averages (av.) and standard deviations (stdev) are based on all the displayed values. Green fields indicate values $>$ (av.+stdev), yellow fields indicate values $<$ (av.-stdev). The values below and to the right of each 48 -fold unit represent the averages of the respective columns or rows for revealing local irregularities. In this case, green fields indicate values $>$ (av. +0.5 stdev), yellow fields indicate values $<$ (av. -0.5 stdev) for an augmented impression. Further abbreviations: $c_{X}$, dry cell mass; TDM, total dry mass; $c_{P}$, riboflavin concentration, determined via HPLC; $V_{R}$, reactor volume. Empty fields indicate that no analytical data were available for the respective reactors.

The data revealed that the relative errors based on the standard deviation were $<5 \%$ for final volumes with an average of $13.5 \mathrm{~mL}$ for 47 reactors and $<7 \%$ for the final riboflavin concentration, for which the average was $9.1 \mathrm{~g} \mathrm{~L}^{-1}$ based on 
HPLC for 45 reactors and the maximum deviation from the average was $12 \%$. The relative error was the same for the total amount of produced riboflavin, which was $122 \mathrm{mg}$ on average in 45 reactors. The relative error on the at-line $\mathrm{pH}$ in 47 reactors was $<2 \%$.

\section{Discussion}

The sequential reproducibility of the riboflavin generation, based on the at-line analysis, was good as long as sufficient oxygen was supplied and feeding was not interrupted. The lower riboflavin concentrations until 32 hours during the eightfold parallel process, represented by crosses in Figure 4-52, may be explained in part by the higher volume compared to the other processes.

The performance of the 48-fold parallel process, during which all the operational parameters for the individual reactors were kept the same (represented with squares in the previous subsection), were accordingly much more homogeneous than during other parallel cultivations with respect to almost all aspects, especially until 38 hours, at which time the process downtime of three hours occurred. After this unplanned incident the results became a little more inhomogeneous, but this was most likely caused by the collapsing gas input after 40 hours, the consecutive accumulation of glucose and the lysis of cells, being accompanied by different base consumption (data not shown). In other words, the parallel reproducibility decreased dramatically when the oxygen input into individual reactors was insufficient because it had an impact on both the riboflavin generation and the course of dry cell mass. The results emphasized again that it was of eminent importance to ensure reliable gas input into the milliliter bioreactors.

Outliers during the portrayed 48 -fold parallel cultivation seemed to be distributed randomly within the setup. Only the final reactor volume seemed to be slightly higher towards the far corners of the reaction block and lower in reactors closer to the air inlet, which was located at the front of the cover lid in the middle between reactors R24 and R32. The possible dependence of reactor volumes from their localization in the reaction block was observed only during this cultivation. It should be further observed in the future. The fact that the total amount of produced riboflavin was the lowest in column 7 and the highest in column 8 of the setup must be coincidental, because there was no difference between these two columns which could serve as a reasonable explanation.

\subsubsection{Summary}

A strategy was developed and implemented, which allowed the fully automated operation of B2 processes in 48 parallel milliliter bioreactors, including intermit- 
tent feed and base addition every $10 \mathrm{~min}$, antifoam addition every $30 \mathrm{~min}$, an intermittent $\mathrm{pH}$ control, and automated at-line analyses for biomass, glucose and riboflavin concentrations. The dissolved oxygen could be monitored online in one column of eight parallel reactors with a prototype of an 8-fold sensor unit. This provided valuable information about the process performance and allowed respective adjustment of the oxygen flow. The oscillations of the dissolved oxygen in the milliliter setup were equivalent to those observed at the laboratory scale.

Sufficient oxygen supply to the process should be granted by the employed gasinducing turbines and by enriching the sterile gas flow with pure oxygen to yield 50-60\% oxygen concentration in the inlet gas flow. However, the gas input into the milliliter reactors collapsed when the hollow axles got stuck (starting mainly after 30-40 hours of the process). The reduced oxygen supply in the affected reactors compromised the riboflavin production and thus reduced the overall parallel reproducibility. These results strongly implied that it was of eminent importance to ensure reliable gas input into the milliliter bioreactors.

Nevertheless, the standard deviation of 45 parallel reactors during a 48 -fold cultivation with equal treatment for all vessels was as low as $7 \%$ for the final riboflavin concentration determined via HPLC, $<6 \%$ for the biomass concentration after 24 hours, and $<5 \%$ for the final reactor volumes. Furthermore, the sequential reproducibility of the $B 2$ process in the milliliter setup was sufficiently accurate to allow the comparison of results obtained from independent parallel cultivations.

The evaporation from the milliliter reactors during B2 processes could be compensated by diluting the additive solutions with water. Furthermore the volumetric increase could be adjusted via the temperature for headspace cooling to meet the profile of the reference B2 process at laboratory scale.

In vessels with sufficient gas supply, the riboflavin generation of $B$. subtilis was in the same range as during continuously fed reference processes at DSM NP. However, the productivity of the finally used Bacillus subtilis RB50::[pRF69 $]_{\mathrm{n}}$ had decreased by approximately $30 \%$ after several passages of the original working cell bank, yielding 9-10 $\mathrm{g} \mathrm{L}^{-1}$ final riboflavin concentration at both scales compared to $13-14 \mathrm{~g} \mathrm{~L}^{-1}$ previously. The corresponding integrated product yields were 0.035 $0.045 \mathrm{~g} \mathrm{~g}^{-1}$ after $48-53$ hours.

Growth of Bacillus subtilis at the milliliter scale was equivalent to that of the intermittently operated reference process at laboratory scale during the first 24 hours. After that there was an indication for slightly lower biomass concentrations, possibly caused by higher oxygen concentrations used for the milliliter cultivations. However, the at-line determined values during this time were not reliable, 
because of a regularly occurring mal-positioning of the light ducts for absorbance measurements in the photometric plate reader.

Due to the very tight schedule for the 48-fold parallel experiments, which required the use of the "multi-pipetting" mode of the lab robot, transfer contaminations between reactors in the same row of the reaction block could not be avoided. Random contaminations with a sporulating bacterium were observed during all parallel cultivations, even when all the added solutions were kept as aseptic as possible and chloramphenicol was added to the batch medium. The origin of these contaminations remained unclear, but their occurrence did not seem to impair the riboflavin productivity. 


\subsection{Guideline suggested for the scale-down of fed-batch processes}

The experience gained during the miniaturization of the B2 process with $B$. subtilis to the automated milliliter setup could be used to propose a guideline for the scale-down of other industrially or scientifically relevant microbial fed-batch processes. A flow chart with the actions suggested for a general iterative adaptation of fed-batch processes to the milliliter system is depicted in Figure 4-54.

Generally, the first step during the scale-down of a process should be the establishment of a reliable, well characterized reference. The process modifications necessary for cultivations in the milliliter setup, particularly the change to an intermittent feeding mode, may have different effects on various microorganisms. Therefore, these influences have to be examined cautiously for each individual process prior to the operation in the screening system. It is recommended to carry out these investigations in a setup at laboratory scale that provides intensive monitoring capabilities, with online monitoring of dissolved oxygen and $\mathrm{pH}$ being the minimum requirement. Furthermore, the detection of biomass, substrate, and (by-)products, in offline samples or online, is of importance for the evaluation of the process performance. Additional off-gas analysis can provide the necessary data for mass balances to support the reliability of the analytical results, particularly for cultivations, during which further gaseous by-products besides carbon dioxide are generated.

The reference process should be carried out at ambient pressure as this will also be the condition in the milliliter reactors. If the original process protocol asks for excess pressure for improved oxygen transfer rates, this should be compensated with oxygen addition to the gas flow. A simple approach for the estimation of the oxygen fraction that yields an equivalent saturated oxygen concentration in the liquid at ambient pressure is the application of Dalton's law (Equation 3-28).

The reference process should provide information about the dynamics of the process, particularly about the maximum oxygen uptake rate and the growth rate during the cultivation. If these are in the range of the B2 process, the length of an appropriate intermittent feeding interval $\Delta t$ will most likely be in the range of five to ten minutes. The addition of feed to 48 parallel reactors takes approximately 90-100 seconds with the "multi-pipetting" mode of the laboratory robot. Depending on the dynamic behavior of the $\mathrm{pH}$ and on the sensitivity of the used microorganisms to $\mathrm{pH}$ shifts, the addition of a fluid for $\mathrm{pH}$ adjustment may have to take place at the same frequency. Therefore, a minimum feeding interval of $4 \mathrm{~min}$ is recommended. The sampling from the reactors in all the six columns of the reaction block for at-line $\mathrm{pH}$ measurements takes 3:30 min, if the pipettes are rinsed with system fluid between the individual sampling steps. In this case, feeding in- 
tervals of at least 7 min have to be chosen. Alternatively, the sampling action can be split and can be carried out in several intervals for separate columns. If disinfection steps for the pipetting needles between each dispensing or sampling action are intended, the indicated feeding interval has to be stretched respectively.

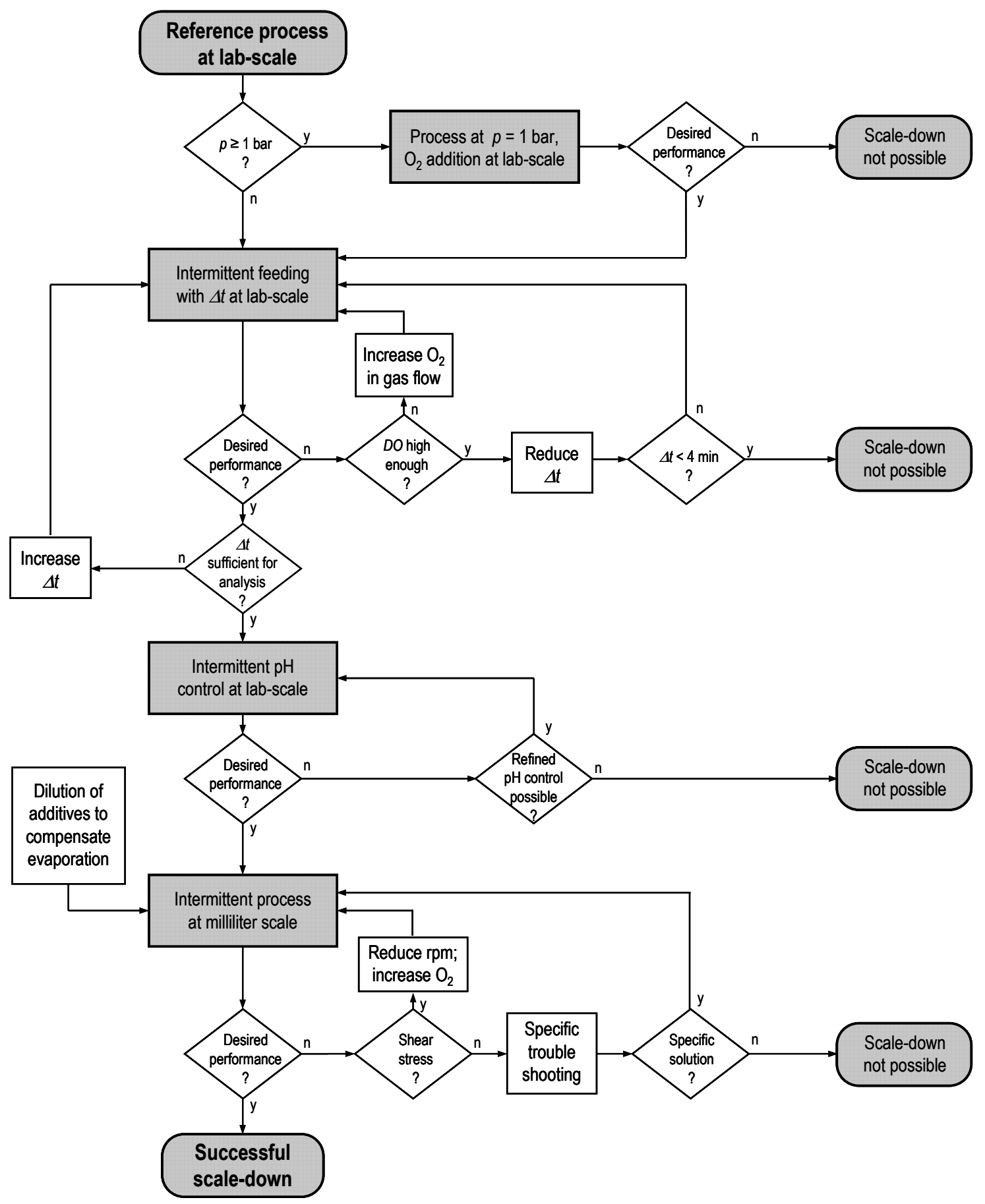

Figure 4-54: Flow chart with suggested guidelines for the iterative scale-down of fed-batch processes, starting from an established reference process at laboratory scale, to the automated milliliter setup, in which cultivations are carried out at ambient pressure ( $p=1 \mathrm{bar})$, oxygen $\left(\mathrm{O}_{2}\right)$ addition to the inlet gas flow ensures sufficiently high dissolved oxygen $(D O)$, feeding is intermittent at feeding intervals $\Delta t \geq 4 \mathrm{~min}$, and $\mathrm{pH}$ is measured at-line and is adjusted intermittently. The stirrer speed (rpm) in the milliliter setup can be adjusted. 
The information about the intended length of the feeding interval is necessary for the next step during the adaptation, which is the operation of the fed-batch process with an intermittent feeding mode at ambient pressure at otherwise unchanged process conditions with respect to the original protocol. If the original protocol provided substrate-limited growth conditions, the cells are now exposed to constant shifts between over- and underfeeding and thus high and low growth rates, according to Monod's model assumptions (Equation 3-12). This becomes more pronounced for longer feeding intervals and may influence the process performance. In addition, the consequent shifts between high and low oxygen uptake rates trigger oscillations of the dissolved oxygen concentration. This can be critical, if the used organisms cannot withstand the peak oxygen tensions.

If the intermittent feeding allows the desired process performance with respect to the reference performance, the $\mathrm{pH}$ control should be shifted to an intermittent mode as well. The frequencies of $\mathrm{pH}$ measurements and $\mathrm{pH}$ adjustments depend again on the process dynamics and have to be adapted respectively. If the cells are too sensitive to the $\mathrm{pH}$ shifts between feed or base additions, this can become a knock-out criterion for the adaptation to the operation in the milliliter setup. In fact, high $\mathrm{pH}$ sensitivity is the only criterion that excludes the milliliter setup as a suitable screening system for a particular process already before any experimental investigations at the reference scale, because the $\mathrm{pH}$ in the milliliter system cannot be controlled continuously yet.

The preceding investigations should allow a successful scale-down of the regarded fed-batch process and thus a successful operation in the automated screening setup. Possibly occurring problems at the milliliter scale may be process-specific and have to be addressed respectively. For example processes, during which fluorescent compounds are generated, can be critical, because the fluorescence-based $\mathrm{pH}$ measurement may be disturbed. In this case, compensation by calculation may be possible, if the impact of the disturbance is quantitatively accessible. Further process-specific challenges, such as a high viscosity or a high particle content of the suspension or an elevated sensitivity of the used organisms to shear stress, may be addressed by dilution of all reaction components (medium, inoculum, additives) or by reduction of the stirrer speed at milliliter scale, respectively. In the latter case, the required oxygen transfer rate should be compensated by an augmented addition of oxygen to the gas flow, if this does not influence the biological system negatively. Regarding the shear stress, the mean volumetric power input in the milliliter reactors is $21.9 \mathrm{~W} \mathrm{~L}^{-1}$ at $2800 \mathrm{rpm}$ for $11.2 \mathrm{~mL}$ according to simulations using computational fluid dynamics (Puskeiler, 2005b). This is similar to the mean power input in stirred tank reactors at laboratory scale. However, the local energy dissipation, particularly close to the edges of the impellers, may differ 
considerably in either system due to the different geometry of the impellers, so that the behavior of the cells in the milliliter vessels cannot be predicted unambiguously.

In addition to the investigations regarding the parameters crucial for a vital process operation, the development of analytical procedures that can be used for the at-line process monitoring at the miniaturized scale may be demanded. Currently the setup is limited to fluorescence and absorbance measurements. An automated separation of cells from the samples is not possible. Logistically the actions of the pipetting robot for sample handling have to be fitted into the periods between automated actions for feeding and $\mathrm{pH}$ control. Therefore, longer feeding intervals make more extensive monitoring possible.

The suggested guideline is based on the results achieved with $B$. subtilis. Hence its application for scale-down of other microbial single cell fed-batch cultivations should be possible with only minor adjustments to specific process requirements being necessary. The transfer of the guideline to processes using filamentous microbial cells or animal cells may not be as simple, mainly due to the high shear sensitivity of these cellular systems. In addition, animal cell culture is much less tolerant to changes in the environment, for example to shifts in $p H$, and it requires different aeration conditions and considerably higher standards regarding the aseptic status of the setup. 


\section{Conclusion}

The challenge of this thesis was the scale-down of a high performance fed-batch production process, operated with $\mathrm{pH}$ control, from a laboratory scale $(3 \mathrm{~L})$ to a milliliter scale $(10 \mathrm{~mL})$ with the goal of achieving a process outcome after the miniaturization equivalent to that at the original scale, especially with respect to productivity and growth. This had never been attempted before.

The production of riboflavin with $B$. subtilis (B2 process) was selected as an exemplary process with industrial relevance. An automated setup with 48 miniaturized stirred tank bioreactors at a milliliter scale was available. Online monitoring of the $\mathrm{pH}$ for a continuous $\mathrm{pH}$ control in the miniature bioreactors was not possible, and the use of a pipetting robot for automated feed and base addition in the milliliter setup made a change from continuous to intermittent feeding and $\mathrm{pH}$ adjustment necessary. Furthermore, the excess pressure, applied for increased oxygen solubility during the original process protocol at laboratory scale, should be compensated with oxygen supply to the gas flow during the operation at ambient pressure in the milliliter vessels. The impact of these operational modifications on the performance of the B2 process had to be investigated. In addition, analytical methods at a microliter scale should be developed for fully automated at-line monitoring of the process in the milliliter setup.

Based on the knowledge gained during the adaptation of the exemplary process, a general guideline for the scale-down of other fed-batch processes to the milliliter setup should be established.

\subsection{Summary of the results}

Initial experiments at laboratory scale revealed that the intermittent feed addition during B2 processes causes temporarily significantly increased oxygen uptake rates which require a higher maximum oxygen supply compared to the continuous feeding mode. This is accompanied by considerable oscillations in dissolved oxygen, $\mathrm{pH}$ and exhaust gas concentrations. Discontinuous feeding alone does not negatively influence the process performance. But elevated oxygen tensions in both continuously and intermittently fed experiments at laboratory scale reduce 
the total biomass generation. The unimpaired riboflavin production during these cultivations indicates an increased specific productivity of the used $B$. subtilis.

Taking the results from the experiments at laboratory scale into consideration, a strategy for the fully automated operation of the B2 process in 48 parallel milliliter bioreactors was developed and implemented. According to this strategy, the feed and base additions are intermittent, with pulses once every $10 \mathrm{~min}$, as well as the $\mathrm{pH}$ control, which is based on at-line $\mathrm{pH}$ measurements in HydroPlates ${ }^{\circledR}$ every 30 min with standard deviations $<0.11 \mathrm{pH}$ units for 48 parallel reactors. Sufficient oxygen supply to the process is granted by gas-inducing impellers and by enriching the sterile gas flow with oxygen to yield 50-60\% oxygen concentration in the inlet gas flow. The dissolved oxygen can be monitored online in one column of eight parallel reactors with a sensor unit prototype. This provides valuable information about the process performance and enables the adjustment of the oxygen flow.

Automated parallel analyses for the at-line monitoring of dry cell mass, glucose and riboflavin concentrations take place every three hours quasi-simultaneously for all 48 reactors using two 96-well microtiter plates, a microplate reader for fluorescence and absorbance, and a microplate washer. Every 24 hours, samples are taken automatically for offline measurements (HPLC and absorbance). Theoretically the offline sampling can take place as often as every three hours.

The riboflavin productivity of Bacillus subtilis RB50::[pRF69] during parallel B2 processes in the milliliter setup is in the same range as during continuously fed standard B2 processes at DSM NP, yielding approximately $10 \mathrm{~g} \mathrm{~L}^{-1}$ final riboflavin concentration when sufficient oxygen is supplied. The standard deviation is $7 \%$ for 45 parallel reactors when all reactors are operated equally. The integrated product yields are $0.035-0.045 \mathrm{~g} \mathrm{~g}^{-1}$ after $48-53$ hours, compared to $0.36 \mathrm{~g} \mathrm{~g}^{-1}$ after 48 hours with the respective strain at DSM NP (Figure 5-1). The growth of $B$. subtilis in the milliliter system is lower compared to the continuously fed process at DSM NP, but is equivalent to the growth during the intermittently operated B2 process at laboratory scale during the first 24 hours. The sequential reproducibility of the processes is sufficiently accurate to allow the comparison of results tested in separate parallel cultivations.

In summary it can be stated, that this is the first time that an industrially relevant fed-batch process has been successfully scaled down to an automated milliliter scale, inclusive of an intermittent $\mathrm{pH}$ control. The performance of the exemplary employed B2 process with Bacillus subtilis RB50::[pRF69] $]_{n}$ in the miniaturized setup is equivalent to that at laboratory scale with respect to product generation, despite the necessary operational modifications. This implies that results regard- 
ing the productivity of this process may be transferred to larger scales and that the automated milliliter setup can be used for screening of various $B$. subtilis clones or process strategies at a large degree of parallelization with little space and few materials required.

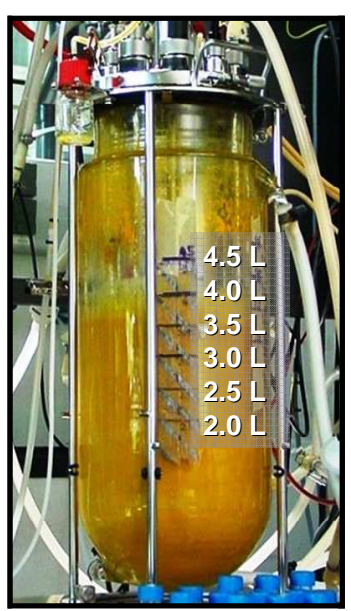

$1 \times 3 L$

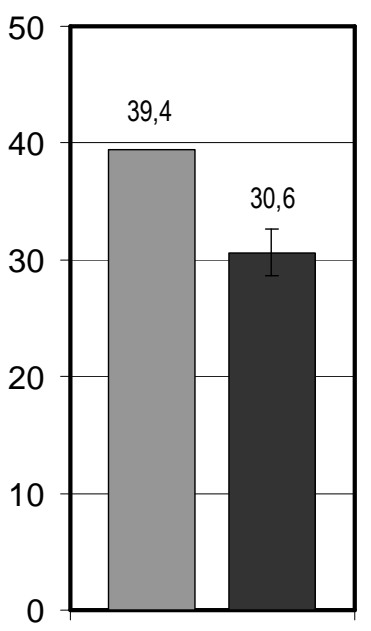

Dry cell mass after $24{\mathrm{~h}, \mathrm{~g} \mathrm{~L}^{-1}}^{-1}$
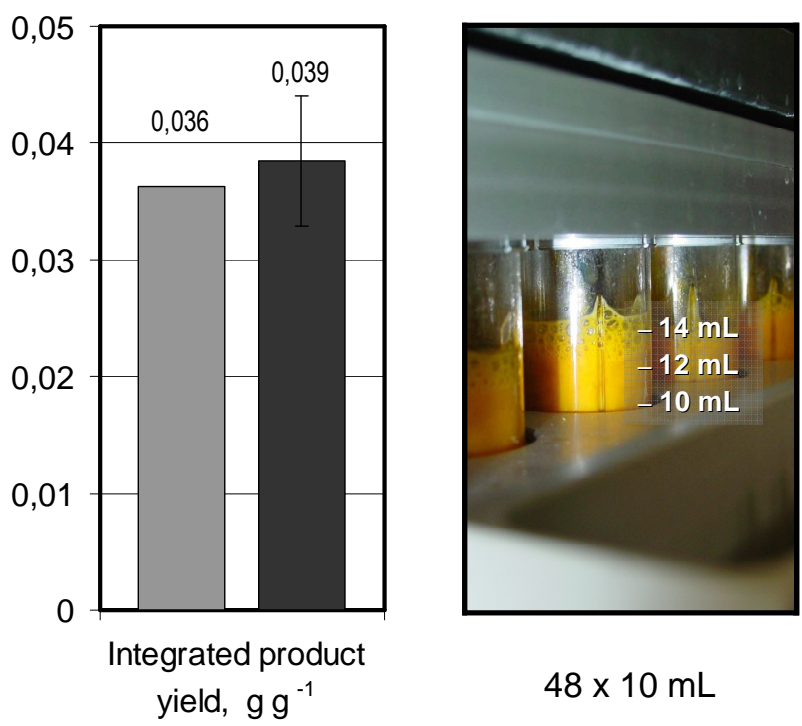

$48 \times 10 \mathrm{~mL}$

Figure 5-1: Dry cell mass concentrations after 24 hours and final integrated riboflavin yields, based on offline data, during a 48-fold parallel B2 process in the milliliter setup (photograph on the right; dark grey bars) or during standard B2 processes at laboratory scale (photograph on the left; light grey bars, based on data from DSM NP).

The scale-down of other fed-batch processes to this setup for enhanced bioprocess development can be undertaken following the guideline that has been established based on the experience gained during this work.

\subsection{Remaining technical concerns}

The gas input into the milliliter reactors collapses when the hollow axles get stuck (starting mainly after 30-40 hours of the process). The reduced oxygen supply in the affected reactors compromises the riboflavin production and thus reduces the overall parallel reproducibility.

The automated at-line analysis for riboflavin is afflicted with large deviations due to four dilution steps of insufficient accuracy with the lab robot, resulting in the need of regular offline analyses at least every 24 hours.

The $p H$ measurement in "HP96L" HydroPlates ${ }^{\circledR}$ is affected by dissolved riboflavin which requires the adjustment of the apparent $\mathrm{pH}$ setpoint profile according to the riboflavin production. However, the used Bacillus does not seem to be negatively 
influenced by $\mathrm{pH}$ values deviating from the desired setpoint. Better $\mathrm{pH}$ sensing and control are necessary for organisms with higher $\mathrm{pH}$ sensitivity.

Due to the very tight schedule for the 48 -fold parallel experiments, which requires the use of the "multi-pipetting" mode of the lab robot, transfer contaminations between reactors in the same row of the reaction block can not be avoided. With the current configuration the system can be used for a maximum number of eight different strains (one per robotic needle). Random contaminations with a sporulating Bacillus are observed during all parallel cultivations, even when all the added solutions are kept as aseptic as possible and chloramphenicol is added to the batch medium. However, these contaminations do not seem to impair the riboflavin productivity.

\subsection{Proposed future work}

The oxygen supply into the milliliter reactors has to be reliable until the end of the process. One approach for an improved gas input may be to widen the inner diameter of the hollow axles so that the gas flow through this channel into the medium is not hindered as easily as inside the currently used axles.

Additional immediate actions may include the revision of the pipetting accuracy for an improved at-line assessment of the riboflavin concentration, and the automation of the sequential increase of the oxygen gas flow during the process as well as the automation of the data evaluation. Stirrer speeds larger than $2700 \mathrm{rpm}$ may enable sufficiently high oxygen transfer rates without raising the oxygen concentration in the gas flow above $50 \%$. Another way to reduce the temporarily elevated dissolved oxygen tensions and thus their impact on the biomass concentration could be an intermittent oxygen addition by an on/off mode corresponding to the intermittent feed addition.

A validation of the system as a tool for screening of new clones or process strategies for the described riboflavin production process should take place. This should include further confirmation of the parallel reproducibility and testing of several Bacillus subtilis strains expressing different riboflavin productivity, possibly with various feeding strategies.

The automated milliliter setup can also be used for other industrially or scientifically relevant fed-batch processes, after testing the possibility of representative scale-down of these processes according to the provided guideline.

Further enhancement of the system will be provided by sensor units for the parallel online measurement and control of the $\mathrm{pH}$ in the milliliter vessels and by professional control software for the screening setup, both of which are currently de- 
veloped (PreSens, Regensburg and DASGIP, Jülich, respectively). An additional major improvement to the setup could be the implementation of a microfluidic solution for the quasi-continuous addition of liquids to the process in order to avoid the massive oscillations and to allow substrate-limited fed-batch operation.

Besides the planned improvement and validation of the described screening apparatus, it is suggested to further investigate if the metabolism in cells, which are exposed to alternating overfeeding and starvation, is altered compared to cells, which are cultivated under constant substrate limitation. Experiments at laboratory scale would provide enough cellular material for transcriptome analysis which could reveal if the intermittent feeding mode triggers the enzymes of the overflow metabolism to remain expressed during the whole course of the cultivation. 


\section{References}

Altenbach-Rehm J, Nell C, Arnold M, Weuster-Botz D. 1999. Parallel bubble columns with fed-batch technique for microbial process development on a small scale. Chem Eng Technol 22(12):1051-1058.

Amanullah A, McFarlane CM, Emery AN, Nienow AW. 2001. Scale-Down Model to Simulate Spatial pH Variations in Large-Scale Bioreactors. Biotechnol Bioeng 73(5):390-399.

Anderlei T, Zang W, Papaspyrou M, Büchs J. 2004. Online respiration activity measurement (OTR, CTR, RQ) in shake flasks. Biochem Eng J 17:187-194.

Arbige MV, Bulthius BA, Schultz J, Crabb D. 1993. Fermentation of Bacillus, in Bacillus subtilis and Other Gram-Positive Bacteria. Sonenshein AL, Hoch JA, Losick R (Eds.). American Society for Microbiology, Washington, D.C.:871-896.

Bacher A, Eberhardt S, Fischer M, Kis K, Richter G. 2000. Biosynthesis of Vitamin B2 (Riboflavin). Annu Rev Nutr 20:153-167.

Bahia D, Cheung R, Buchs M, Geisse S, Hunt I. 2005. Optimisation of insect cell growth in deep-well blocks: development of a high-throughput insect cell expression screen. Protein Expr Purif 39:61-70.

Baumann WH, Lehmann M, Schwinde A, Ehret R, Brischwein M, Wolf B. 1999. Microelectronic sensor system for microphysiological application on living cells. Sensors and Actuators1 B 55:77-89.

Bellgardt $\mathrm{KH}$. Introduction, in Bioreaction Engineering: Modelling and Control. Schügerl K, Bellgardt KH (Eds.). Springer-Verlag, Berlin Heidelberg. 1-18.

Bhargava S, Wenger KS, Marten MR. 2003. Pulsed Addition of Limiting-Carbon During Aspergillus oryzae Fermentation Leads to Improved Productivity of a Recombinant Enzyme. Biotechnol Bioeng 82(1):111-117. 
Bhargava S, Wenger KS, Rane K, Rising V, Marten MR. 2005. Effect of Cycle Time on Fungal Morphology, Broth Rheology, and Recombinant Enzyme Productivity During Pulsed Addition of Limiting Carbon Source Biotechnol Bioeng 89(5):524529.

Biehl S. 2004. Automatisierung eines Produktionsprozesses mit Bacillus subtilis in parallelen Bioreaktoren im mL-Maßstab. Diplomarbeit, TU München.

Bretzel W, Schurter W, Ludwig B, Kupfer E, Doswald S, Pfister M, Van Loon APGM. 1999. Commercial riboflavin production by recombinant Bacillus subtilis: downstream processing and comparison of the composition of riboflavin produced by fermentation or chemical synthesis. J Indus Microbiol Biotechnol 22:19-26.

Brischwein M, 2003. Personal communications.

Brockhaus (2000). Der Brockhaus in fünf Bänden (5). F.A. Brockhaus GmbH, Leipzig:4940.

Büchs J. 2001. Introduction to advantages and problems of shaken cultures. Biochem Eng J 7:91-98.

Bylund F, Collet E, Enfors SO, Larsson G. 1998. Substrate gradient formation in the large-scale bioreactor lowers cell yield and increases by-product formation. Bioprocess Eng 18:171-180.

Bylund F, Guillard F, Enfors SO, Trägårdh C, Larsson G. 1999. Scale down of recombinant protein formation: a comparative study of scaling performance. Bioprocess Eng 20:377-389.

Chartrain M, Salmon P, Robinson D, Buckland B. 2000. Metabolic engineering and directed evolution for the production of pharmaceuticals. Curr Opin Biotechnol 11:209-214.

Coquard D, Huecas M, Ott M, van Dijl JM, Van Loon APGM, and Hohmann HP. 1997. Molecular cloning and characterisation of the ribC gene from Bacillus subtilis: a point mutation in ribC results in riboflavin overproduction. $\mathrm{Mol}$ Gen Genet 254:81-84.

Danielson PB, Büchs J, Stöckmann C, Fogleman JC. 2004. Maximizing cell densities in miniprep-scale cultures with $\mathrm{H} 15$ medium and improved oxygen transfer. Biochem Eng J 17:175-180.

Dauner M, Sauer U. 2001. Stoichiometric Growth Model for Riboflavin-Producing Bacillus subtilis. Biotechnol Bioeng 76(2):132-143. 
Dauner M, Bailey JE, Sauer U. 2001a. Metabolic Flux Analysis with a Comprehensive Isotopomer Model in Bacillus subtilis. Biotechnol Bioeng 76(2):144-156.

Dauner M, Storni T, Sauer U. 2001b. Bacillus subtilis Metabolism and Energetics in Carbon-Limited and Excess-Carbon Chemostat Culture. J Bacteriol 183(24):73087317.

Debabov VG. 1982. The industrial use of bacilli, in The molecular biology of bacilli (vol 1). Dubnau DA (Ed.). Academic Press Inc., New York:331-370.

De Wulf P, Vandamme EJ. 1997. Production of D-ribose by fermentation. Appl Microbiol Biotechnol 48: 141-148

Dilsen S, Paul W, Herforth D, Sandgathe A, Altenbach-Rehm J, Freudl R, Wandrey C, Weuster-Botz D. 2001. Evaluation of parallel operated small-scale bubble columns for microbial process development using Staphylococcus carnosus. J Biotechnol 88(1):77-84.

Doig SD, Diep A, Baganz F. 2005. Characterisation of a novel miniaturised bubble column bioreactor for high throughput cell cultivation. Biochem Eng J 23:97-105.

Duetz WA, Rüedi L, Hermann R, Minas W, O'Connor K, Büchs J, Witholt B. 2000. Methods for intense aeration, growth, storage, and replication of bacterial strains in microtiter plates. Appl Environ Microb 66(6):2641-2646.

Duetz WA, Witholt B. 2004. Oxygen transfer by orbital shaking of square vessels and deepwell microtiter plates of various dimensions. Biochem Eng J 17:181-185.

Elmahdi I, Baganz F, Dixon K, Harrop T, Sugden D, Lye GJ. 2003. pH control in microwell fermentations of $S$. erythraea CA340: influence on biomass growth kinetics and erythromycin biosynthesis. Biochem Eng J 16:299-310.

Enfors SO, Jahic M, Rozkov A, Xu B, Hecker M, Jürgen B, Krüger E, Schweder T, Hamer G, O'Beirne D, Noisommit-Rizzi N, Reuss M, Boone L, Hewitt $C$, McFarlane $\mathrm{C}$, Nienow A, Kovacs $\mathrm{T}$, Trägårdh $\mathrm{C}$, Fuchs L, Revstedt J, Friberg PC, Hjertager B, Blomsten G, Skogman H, Hjort S, Hoeks F, Lin HY, Neubauer P, Van der Lans R, Luyben K, Vrabel P, Manelius A. 2001. Physiological responses to mixing in large scale bioreactors. J Biotechnol 85:175-185.

Ferrari E, Jarnagin AS, Schmidt BF. 1993. Commercial Production of Extracellular Enzymes, in Bacillus subtilis and Other Gram-Positive Bacteria. Sonenshein AL, Hoch JA, Losick R (Eds.). American Society for Microbiology, Washington, D.C.:917-938. 
Fischer E, Sauer U. 2005. Large-scale in vivo flux analysis shows rigidity and subotimal performance of Bacillus subtilis metabolism. Nat Genet 37:636-640.

Freyer S, König M, Künkel A. 2004. Validating shaking flasks as representative screening system. Biochem Eng J 17:169-173.

Fuhrer T, Fischer E, Sauer U. 2005. Experimental Identification and Quantification of Glucose Metabolism in Seven Bacterial Species. J Bacteriol 187(5):1581-1590.

Geipel K. 2002. Fluorimetrische Bestimmung von Glucose in zellhaltigen Proben im $\mu \mathrm{L}-$ Maßstab. Diplomarbeit, TU München.

George S, Larsson G, Enfors SO. 1993. A scale-down two-compartment reactor with controlled substrate oscillations: Metabolic response of Saccharomyces cerevisiae. Bioprocess Eng 9(6):249-257.

Gershanovich VN, Kukanova AI, Galushkina ZM, Stepanov AI. 2000. Transketolase mutation in riboflavin-synthesizing strains of Bacillus subtilis. Mol Gen Mikrobiol Virusol 3:3-7.

Girard P, Jordan M, Tsao M, Wurm FM. 2001. Small-scale bioreactor system for process development and optimization. Biochem Eng J 7:117-119.

Harwood CR, Wipat A. 1996. Sequencing and functional analysis of the genome of Bacillus subtilis strain 168. FEBS Lett 389:84-87.

Hewitt CJ, Nebe-Von Caron G, Axelsson B, McFarlane CM, Nienow A. 2000. Studies related to the Scale-Up of High-Cell-Density E. coli Fed-Batch Fermentations Using Multiparameter Flow-Cytometry: Effect of a Changing Microenvironment with Respect to Glucose and Dissolved Oxygen Concentration. Biotechnol Bioeng 70(4):381-390.

Hibbert EG, Baganz F, Hailes HC, Ward JM, Lye GJ, Woodley JM, Dalby PA. 2005. Directed evolution of biocatalytic processes. Biomol Eng 22:11-19.

Hohmann P. 2004. Personal communications.

Hümbelin M, Griesser V, Keller T, Schurter W, Haiker M, Hohmann HP, Ritz H, Richter G, Bacher A, Van Loon APGM. 1999. GTP cyclohydrolase II and 3,4dihydroxy-2-butanone 4-phosphate synthase are rate-limiting enzymes in riboflavin synthesis of an industrial Bacillus subtilis strain used for riboflavin production. J Indust Microbiol Biotechnol 22:1-7. 
Illarionov B, Eisenreich W, Bacher A. 2001. A pentacyclic reaction intermediate of riboflavin synthase. PNAS 98(13):7224-7229.

Jain MK, Cai Q, Grimes CA. 2001. A wireless micro-sensor for simultaneous measurement of pH, temperature, and pressure. Smart Mater Struct 10:347-353.

John GT, Klimant I, Wittmann C, Heinzle E. 2003. Integrated optical sensing of dissolved oxygen in microtiter plates: a novel tool for microbial cultivation. Biotechnol Bioeng 81(7):829-836.

Karos M, Vilarino C, Bollschweiler C, Revuelta JL. 2004. A genome-wide transcription analysis of a fungal riboflavin overproducer. J Biotechnol 113:69-76.

Knepper A. 2003. Intermittent Substrate Feeding during Fed-Batch Fermentations with B. subtilis. Diplomarbeit, TU München.

Koizumi S, Yonetani Y, Maruyama A, Teshiba S. 2000. Production of riboflavin by metabolically engineered Corynebacterium ammoniagenes. Appl Microbiol Biotechnol 53:674-679.

Konz JO, King J, Cooney CL. 1998. Effects of oxygen on recombinant protein expression. Biotechnol Prog 14(3):393- 409.

Kovács T, Hargitai A, Kovács KL, Mécs I. 1998. pH-dependent activation of the alternative transcriptional factor $\sigma^{\mathrm{B}}$ in Bacillus subtilis. FEMS Microbiol Lett 165:323-328.

Kovárová-Kovar K, Egli T. 1998. Growth Kinetics of Suspended Microbial Cells: From Single-Substrate-Controlled Growth to Mixed-Substrate Kinetics. Microbiol Mol Biol Rev 62(3):646-666.

Lamping SR, Zhang H, Allen B, Shamlou PA. 2003. Design of a prototype miniature bioreactor for high throughput automated bioprocessing. Chem Eng Sci 58(36): 747-758.

Lin HY, Neubauer P. 2000. Influence of controlled glucose oscillations on a fedbatch process of recombinant Escherichia coli. J Biotechnol 79:27-37.

Lye GJ, Ayazi-Shamlou P, Baganz F, Dalby PA, Woodley JM. 2003. Accelerated design of bioconversion processes using automated microscale processing techniques. Trends Biotechnol 21(1):29-37. 
Mack M, Van Loon APGM, Hohmann HP. 1998. Regulation of Riboflavin Biosynthesis in Bacillus subtilis Is Affected by the Activity of the Flavokinase/Flavin Adenine Dinucleotide Synthetase Encoded by ribC. J Bacteriol 180(4):950-955.

Maharbiz MM, Holtz WJ, Howe RT, Keasling JD. 2004. Microbioreactor arrays with parametric control for high-throughput experimentation. Biotechnol Bioeng 85(4):376-381.

Marss B, Delgarve S, Murphy D. 1999. Novel approaches for discovering industrial enzymes. Curr Opin Microbiol 2:241-245.

März U. 2005. GA-103R World Markets for Fermentation Ingredients. Business Communications Company Inc., Connecticut, USA.

Monod J. 1949. The growth of bacterial cultures. Ann Rev Microbiol 3:371-378.

Nakajima K. 2004. Effects of riboflavin on 6,7-dimethyl-8-ribityllumazine formation in growing cells of a riboflavin-adenine-deficient mutant, Bacillus subtilis. J Nutr Sci Vitaminol (Tokyo) 50(5):377-379.

Neubauer P, Ånman M, Tørnkvist M, Larsson G, Enfors SO. 1995. Response of guanosine tetraphosphate to glucose fluctuations in fed-batch cultivations of Escherichia coli. J Biotechnol 43:195-204.

Onyeaka H, Nienow A, Hewitt CJ. 2003. Further Studies related to the Scale-Up of High-Cell-Density E. coli Fed-Batch Fermentations: The Additional Effect of a Changing Microenvironment When Using Aqueous Ammonia to Control pH. Biotechnol Bioeng 84(4):474-484.

Oosterhuis NMG, Kossen NWF, Olivier APC, Schenk ES. 1985. Scale-Down and Optimization Studies of the Gluconic Acid Fermentation by Gluconobacter Oxydans. Biotechnol Bioeng 27:711-720.

Papagianni M, Mattey M, Kristiansen B. 2003. Design of a Tubular Loop Bioreactor for Scale-up and Scale-down of Fermentation Processes. Biotechnol Prog 19:14981502.

Parekh S, Vinci VA, Strobel RJ. 2000. Improvement of microbial strains and fermentation processes. Appl Microbiol Biotechnol. 54:287-301.

Perkins JB, Pero JG. 1993. Biosynthesis of Riboflavin, Biotin, Folic Acid, and Cobalamin, in Bacillus subtilis and Other Gram-Positive Bacteria. Sonenshein AL, Hoch JA, Losick R (Eds.). American Society for Microbiology, Washington, D.C.: 319-334. 
Perkins J, Sloma A, Hermanm T, Thieriault K, Zachgo E, Erdenberger T, Hannet N, Chatterjee N, Williams V, Rufo G, Hatch R, Pero J. 1999. Genetic engineering of Bacillus subtilis for the commercial production of riboflavin. J Indus Microbiol Biotechnol 22:8-12.

Pero J, Sloma A. 1993. Proteases, in Bacillus subtilis and Other Gram-Positive Bacteria. Sonenshein AL, Hoch JA, Losick R (Eds.). American Society for Microbiology, Washington, D.C.:939:952.

PreSens. 2003. Manual Gebrauchsanleitung für HydroPlate ${ }^{\circ}$ HP96T, HydroPlate $®$ HP96L, HydroPlate ${ }^{\circledR}$ HP96C. PreSens Precision Sensing GmbH, Regensburg, Germany.

Priest FG. 1993. Systematics and Ecology of Bacillus, in Bacillus subtilis and Other Gram-Positive Bacteria. Sonenshein AL, Hoch JA, Losick R (Eds.). American Society for Microbiology, Washington, D.C.:3-16.

Puskeiler R. 2004. Miniaturisierte Parallelreaktoren zur Hochdurchsatz-Bioprozessentwicklung. Dissertation, TU München.

Puskeiler R, Weuster-Botz D. 2004. Rührkesselreaktoren im mL-Maßstab: Kultivierung von Escherichia coli. Chem Ing Tech 76(12):1865-1869.

Puskeiler R, Kaufmann K, Weuster-Botz D. 2005a. Development, Parallelization, and Automation of a Gas-Inducing Milliliter-Scale Bioreactor for High-Throughput Bioprocess Design (HTBD). Biotechnol Bioeng 89(5):512-523.

Puskeiler R, Kusterer A, John G, Weuster-Botz D. 2005b. Miniature bioreactors for automated high-throughput bioprocess design (HTBD): Reproducibility of parallel fed-batch cultivations with Escherichia coli. Biotechnol Appl Biochem DOI: $10.1042 / B A 20040197$.

Reuss M. 1993. Oxygen Transfer and Mixing: Scale-Up Implications, in Biotechnology (vol. 3). Rehm HJ, Reed G (Eds.). VCH Verlagsgesllschaft, Weinheim:185217.

Ruan C, Ong KG, Mungle C, Paulose M, Nickl NJ, Grimes CA. 2003. A wireless pH sensor based on the use of salt-independent micro-scale polymer spheres. Sens Actuators B 96:61-69.

Sauer U, Hatzimanikatis V, Hohmann HP, Manneberg M, Van Loon AP, Bailey JE. 1996. Physiology and metabolic fluxes of wild-type and riboflavin-producing Bacillus subtilis. Appl Environ Microbiol 62:3687-3696. 
Sauer U, Hatzimanikatis V, Bailey JE, Hochuli M, Szyperski T, Wüthrich K. 1997. Metabolic fluxes in riboflavin-producing Bacillus subtilis. Nature Biotechnol $15: 448-452$.

Sauer U, Cameron DC, Bailey JE. 1998. Metabolic capacity of Bacillus subtilis for the production of purine nucleosides, riboflavin, and folic acid. Biotechnol Bioeng 59:227-238.

Schallmey M, Singh A, Ward OP. 2004. Developments in the use of Bacillus species for industrial production. Can J Microbiol 50: 1-17.

Schlee D, Kleber HP (Eds.). 1991. Wörterbücher der Biologie: Biotechnologie. Gustav Fischer Verlag Jena:238.

Schumpe A, Quicke G, Deckwer WD. 1982. Gas solubilities in microbial media. Adv Biochem Eng 24:1-38.

Schweder T, Krüger E, Xu B, Jürgen B, Blomsten G, Enfors SO, Hecker M. 1999. Monitoring of genes that respond to process related stress in large scale bioprocesses. Biotechnol Bioeng 65:151-159.

Shuler ML, Kargi F. 1992. Bioprocess Engineering. Prentice Hall, Englewood Cliffs, NJ.

Solf A. 2003. Messung des $\mathrm{pH}$-Wertes in $\mathrm{mL}$-Bioreaktoren und seine Regelung über Titration mit Ammoniumhydroxid. Diplomarbeit, TU München.

Solovieva IM, Kreneva RA, Leak DJ, Perumov DA. 1999. The ribR gene encodes a monofunctional riboflavin kinase which is involved in regulation of the Bacillus subtilis riboflavin operon. Microbiology 145:67-73.

Solovieva IM, Kreneva RA, Errais Lopes L, Perumov DA. 2005. The riboflavin kinase encoding gene ribR of Bacillus subtilis is a part of a $10 \mathrm{~kb}$ operon, which is negatively regulated by the yrzC gene product. FEMS Microbiol Lett 243(1):51-58.

Sonenshein AL. 1993. Introduction to Metabolic Pathways, in Bacillus subtilis and Other Gram-Positive Bacteria. Sonenshein AL, Hoch JA, Losick R (Eds.). American Society for Microbiology, Washington, D.C.:127-132.

Stahmann KP, Revuelta JL, Seulberger H. 2000. Three biotechnical processes using Ashbya gossypii, Candida famata, or Bacillus subtilis compete with chemical riboflavin production. Appl Microbiol Biotechnol 53:509-516. 
Teich A, Meyer S, Lin HY, Andersson L, Enfors SO, Neubauer P. 1999. Growth rate related concentration changes of the starvation response regulators sigma (S) and ppGpp in glucose-limited fed-batch and continuous cultures of Escherichia coli. Biotechnol Prog 15(1):123-129.

Thiry M, Cingolani D. 2002. Optimizing scale-up fermentation processes. Trends Biotechnol 20(3):103-105.

Van Loon APGM, Hohmann HP, Bretzel W, Hümbelin M, Pfister M. 1996. Development of a Fermentation Process fort he Manufacture of Riboflavin. Chimia 50(9):410-412.

Weuster-Botz D. 1999. Medienoptimierung mit Genetischem Algorithmus, in Die Rolle der Reaktionstechnik in der mikrobiellen Verfahrensentwicklung. Forschunszentrum Jülich $\mathrm{GmbH}$, Jülich:37-45.

Weuster-Botz D, Altenbach-Rehm J, Arnold M. 2001a. Parallel substrate feeding and $\mathrm{pH}$-control in shaking-flasks. Biochem Eng $\mathrm{J}$ 7(2):163-170.

Weuster-Botz D, Altenbach-Rehm J, Hawrylenko A. 2001b. Process-engineering characterization of small-scale bubble columns for microbial process development. Bioproc Biosys Eng 24:3-11.

Weuster-Botz D, Stevens S, Hawrylenko A. 2002. Parallel-operated stirred columns for microbial process development. Biochem Eng J 11:69-72.

Weuster-Botz D. 2005. Parallel Reactor Systems for Bioprocess Development. Adv Biochem Engin/Biotechnol 92:125-143.

Wittmann C, Kim HM, John G, Heinzle E. 2003. Characterization and application of an optical sensor for quantification of dissolved $\mathrm{O}_{2}$ in shake-flasks. Biotechn Letters 25:377-380.

Yang X, Ong KG, Dreschel WR, Zeng K, Mungle CS, Grimes CA. 2002. Design of a Wireless Sensor Network for Long-term, In-Situ Monitoring of an Aqueous Environment. Sensors 2:455-472.

Young M. 1984. Gene amplification in Bacillus subtilis. J Gen Microbiol 130 (7):1613-1621.

Zamboni N, Mouncey N, Hohmann HP, Sauer U. 2003. Reducing maintenance metabolism by metabolic engineering of respiration improves riboflavin production by Bacillus subtilis. Metabol Eng 5:49-55. 
Zanzotto A, Szita N, Boccazzi P, Lessard P, Sinskey AJ, Jensen KF. 2004. Membrane-Aerated Microbioreactor for High-Throughput Bioprocessing. Biotechnol Bioeng 87(2):243-254.

Zeng AP. 1995. Effect of $\mathrm{CO}_{2}$ absorption on the measurement of $\mathrm{CO}_{2}$ evolution rate in aerobic and anaerobic continuous cultures. Appl Microbiol Biotechnol 42:688-691. 


\section{Appendix}

The appendix provides an explanation of the used symbols and abbreviations (7.1) and a detailed compilation of the applied materials and methods (7.2), followed by related information (7.3).

\subsection{Abbreviations and symbols}

\begin{tabular}{|c|c|}
\hline Abbreviation & Denotation \\
\hline AF $1-4$ & antifoam solutions 1 to 4 (for details refer to 7.2.2.) \\
\hline Amplex Red ${ }^{\circledR}$ & brand name for fluorescent dye 10-acetyl-3,7-dihydroxyphenoxazin \\
\hline$A z^{r}$ & resistance to purine-analog 8-azaguanine \\
\hline B2 process & riboflavin (vitamin B2) production process with Bacillus subtilis \\
\hline B. subtilis & Bacillus subtilis strain RB50::[pRF69 $]_{\mathrm{n}}$ \\
\hline$D c^{r}$ & resistance to purine-analog decoyinine \\
\hline DF & dilution factor \\
\hline DSM NP & DSM Nutritional Products \\
\hline HP96L & HydroPlate ${ }^{\circledR}$ HP96L for parallel pH measurement \\
\hline MFC & mass flow controller \\
\hline MTP & microtiter plate \\
\hline PPP & pentose phosphate pathway \\
\hline pRF69 & riboflavin-producing plasmid 69 \\
\hline pRF93 & riboflavin-producing plasmid 93 \\
\hline $\mathrm{RoF}^{r}$ & resistance to riboflavin-analog roseoflavin \\
\hline TCA cycle & tricarbonic acid cycle \\
\hline TUM & Technische Universität München \\
\hline rpm & revolutions per minute \\
\hline Chemical & Denotation \\
\hline AMP & adenosine monophosphate \\
\hline DMRL & 6,7-dimethyl-8-ribityllumazine \\
\hline DMSO & dimethyl sulfoxide \\
\hline EMS & ethyl methyl sulfonate \\
\hline FAD & flavin adenine dinucleotide \\
\hline FMN & flavin mononucleotide \\
\hline GOD & glucose oxidase \\
\hline GTP & guanosine triphosphate \\
\hline $\mathrm{H}_{2} \mathrm{O}_{2}$ & hydrogen peroxide \\
\hline
\end{tabular}




\begin{tabular}{ll} 
HRP & horseradish peroxidase \\
IMP & inosine monophosphate \\
$\mathrm{MS}$ & methionine sulfoxide \\
$\mathrm{NADH}$ & nicotine adenine dinucleotide (reduced form) \\
$\mathrm{NaOH}$ & sodium hydroxide \\
$\mathrm{NaP}_{\mathrm{i}}$ & sodium phosphate \\
$\mathrm{NH}_{4} \mathrm{OH}$ & ammonium hydroxide \\
$\mathrm{PEEK}$ & polyether ether ketone \\
$\mathrm{PMMA}$ & poly(methyl methacrylate) \\
$\mathrm{PS}$ & polystyrene \\
\hline
\end{tabular}

\begin{tabular}{|c|c|c|}
\hline Symbol & Units & Denotation \\
\hline$a$ & $\mathrm{~m}^{2} \mathrm{~m}^{-3}$ & volumetric interfacial mass transfer area \\
\hline $\mathrm{ABSO}_{444}$ & - & absorbance at $444 \mathrm{~nm}$ \\
\hline CER & $\mathrm{mol} \mathrm{L}^{-1} \mathrm{~s}^{-1}$ & carbon dioxide evolution rate \\
\hline$c_{i}$ & $g^{-1}$ & concentration of component $\mathrm{i}$ \\
\hline$c_{O}$ & $\mathrm{~mol} \mathrm{~L}^{-1}$ or $\mathrm{g} \mathrm{L}^{-1}$ & oxygen concentration in the liquid bulk \\
\hline$c_{O}^{*}$ & $\mathrm{~mol} \mathrm{~L}^{-1}$ or g $\mathrm{L}^{-1}$ & saturated oxygen concentration at the gas/liquid interface \\
\hline$c_{P}$ & $g L^{-1}$ & riboflavin (generally product) concentration \\
\hline$c_{S}$ & $g^{-1}$ & glucose (generally substrate) concentration \\
\hline$c_{S, i n}$ & $g^{-1}$ & substrate concentration in the feed solution \\
\hline$c_{x}$ & $\mathrm{~g} \mathrm{~L}^{-1}$ & dry cell mass (= biomass) concentration \\
\hline$c_{x 0}$ & $g^{-1}$ & dry cell mass concentration at $t=0$ \\
\hline DO & \%air saturation & $\begin{array}{l}\text { dissolved oxygen as a fraction of the oxygen concentra- } \\
\text { tion in the liquid phase at saturation with air }\end{array}$ \\
\hline$d_{R}$ & $\mathrm{~m}$ & stirrer diameter \\
\hline$I D$ & $\mathrm{~m}$ & inner diameter (pump tubing) \\
\hline$I_{\max }$ & - & constant for $p H$ calibration curve in HydroPlates ${ }^{\circledR}$ \\
\hline$I_{\min }$ & - & constant for $p H$ calibration curve in HydroPlates ${ }^{\circledR}$ \\
\hline$I_{R}$ & - & referenced fluorescence signal in HydroPlates ${ }^{\circledR}$ \\
\hline$k_{g}$ & $\mathrm{~m} \mathrm{~s}^{-1}$ & oxygen transfer coefficient in the gas phase \\
\hline$K_{H, O}$ & $\mathrm{~mol} \mathrm{~m}^{-3}$ bar $^{-1}$ & Henry constant for oxygen \\
\hline$k_{L}$ & $\mathrm{~m} \mathrm{~s}^{-1}$ & oxygen transfer coefficient in the liquid phase \\
\hline$k_{\llcorner} a$ & $s^{-1}$ & volumetric oxygen transfer coefficient \\
\hline$K_{D}^{*}$ & L & $\begin{array}{l}\text { empiric constant for the intermittent } \mathrm{pH} \text { control algorithm } \\
\text { (differential fraction) }\end{array}$ \\
\hline$K_{P}$ & L & $\begin{array}{l}\text { empiric constant for the intermittent } \mathrm{pH} \text { control algorithm } \\
\text { (proportional fraction) }\end{array}$ \\
\hline$K_{S}$ & $g^{-1}$ & saturation constant of the limiting substrate \\
\hline$m_{S}$ & $g_{\text {substrate }} g_{\text {cells }}{ }^{-1} s^{-1}$ & maintenance coefficient \\
\hline
\end{tabular}




\begin{tabular}{|c|c|c|}
\hline MW & $\mathrm{g} \mathrm{mol}^{-1}$ & molecular weight \\
\hline$M W_{O}$ & $\mathrm{~g} \mathrm{~mol}^{-1}$ & molecular weight of oxygen \\
\hline$O D$ & - & optical density \\
\hline$O D_{650}$ & - & optical density at $650 \mathrm{~nm}$ \\
\hline OTR & $\mathrm{mol} \mathrm{L}^{-1} \mathrm{~s}^{-1}$ & oxygen transfer rate \\
\hline OUR & $\mathrm{mol} \mathrm{L}^{-1} \mathrm{~s}^{-1}$ & oxygen uptake rate \\
\hline$p_{O}$ & bar & partial pressure of oxygen in the gas phase \\
\hline$p_{O}^{*}$ & bar & $\begin{array}{l}\text { partial pressure of oxygen at the gas/liquid interface at } \\
\text { saturation }\end{array}$ \\
\hline $\mathrm{pH}_{0}$ & - & constant for $p H$ calibration curve in HydroPlates ${ }^{\circledR}$ \\
\hline$p H_{\text {set }}$ & - & $p H$ setpoint \\
\hline$q_{0}$ & $g_{\text {oxygen }} g_{\text {cells }}^{-1} s^{-1}$ & specific oxygen uptake rate \\
\hline$q_{P}$ & $g_{\text {product }} g_{\text {cells }}{ }^{-1} \mathrm{~s}^{-1}$ & specific production rate \\
\hline$q_{s}$ & $g_{\text {substrate }} g_{\text {cells }}{ }^{-1} s^{-1}$ & specific substrate uptake rate \\
\hline$q_{S, m}$ & $g_{\text {substrate }} g_{\text {cells }}{ }^{-1} s^{-1}$ & specific substrate uptake rate for maintenance \\
\hline$q_{S, \max }$ & $g_{\text {substrate }} g_{\text {cells }}{ }^{-1} s^{-1}$ & maximum specific substrate uptake rate \\
\hline$q_{S, P}$ & $g_{\text {substrate }} g_{\text {cells }}{ }^{-1} s^{-1}$ & specific substrate uptake rate for production \\
\hline$q_{S, \mu}$ & $g_{\text {substrate }} g_{\text {cells }}{ }^{-1} s^{-1}$ & specific substrate uptake rate for growth \\
\hline$r_{i}$ & $\mathrm{~g} \mathrm{~g}^{-1} \mathrm{~s}^{-1}$ & specific reaction rate of component $i$ \\
\hline$R Q$ & $\mathrm{~mol}_{\mathrm{CO} 2} \mathrm{~mol}_{\mathrm{O} 2}^{-1}$ & respiratory coefficient $(=C E R / O U R)$ \\
\hline$t$ & $s$ & (process) time \\
\hline$T$ & ${ }^{\circ} \mathrm{C}$ & temperature \\
\hline$T D M$ & $\mathrm{~g} \mathrm{~L}^{-1}$ & total dry mass \\
\hline$V_{\text {base }}$ & L & base volume to be added according to $\mathrm{pH}$ control \\
\hline$V_{0}$ & L & initial reactor volume \\
\hline$V_{R}$ & L & actual reactor volume \\
\hline$V_{\text {total }}$ & $\mathrm{L}$ & total reactor volume $\left(=V_{R}+\Sigma\right.$ sample volume $)$ \\
\hline$\dot{V}_{g}$ & $\mathrm{Ls}^{-1}$ & gas flow \\
\hline$\dot{V}_{g, \text { in }}$ & $\mathrm{Ls}^{-1}$ & inlet gas flow \\
\hline$\dot{V}_{g, \text { out }}$ & $\mathrm{Ls}^{-1}$ & outlet gas flow \\
\hline$\dot{V}_{\text {in }}$ & $\mathrm{Ls}^{-1}$ & inflow rate of feed solution \\
\hline$\dot{V}_{\text {out }}$ & $\mathrm{Ls}^{-1}$ & outflow rate \\
\hline$Y_{P / S}$ & griboflavin $_{\text {glucose }}{ }^{-1}$ & integrated product yield on substrate \\
\hline$Y_{X / S}$ & $g_{\text {cell mass }} g_{\text {glucose }}{ }^{-1}$ & integrated growth yield on glucose \\
\hline$Y_{P S^{-1}}^{*}$ & $g_{\text {product }} g_{\text {substarte }}{ }^{-1}$ & differential product yield coefficient on substrate \\
\hline$Y_{X / O}^{*}$ & $g_{\text {cell mass }} g_{\text {oxygen }}{ }^{-1}$ & differential growth yield coefficient on oxygen \\
\hline$Y_{X / S}^{*}$ & $\mathrm{~g}_{\text {cell mass }} \mathrm{g}_{\text {substrate }}{ }^{-1}$ & differential growth yield coefficient on substrate \\
\hline
\end{tabular}




\begin{tabular}{lcl}
\hline Greek symbol & Units & Denotation \\
\hline$\gamma_{O, \text { in }}$ & $\%$ & molar fraction of oxygen in the inlet gas flow \\
$\gamma_{O, \text { out }}$ & $\%$ & molar fraction of oxygen in the outlet gas flow \\
$\lambda$ & $\mathrm{m}$ & Wavelength \\
$\lambda_{\text {emission }}$ & $\mathrm{m}$ & wavelength for fluorescence emission \\
$\lambda_{\text {excitation }}$ & $\mathrm{m}$ & wavelength for fluorescence excitation \\
$\mu$ & $\mathrm{s}^{-1}$ & specific growth rate \\
$\mu_{\max }$ & $\mathrm{s}^{-1}$ & maximum specific growth rate \\
\hline
\end{tabular}

\section{Remarks regarding "non-SI units"}

$\mathrm{L}$

In Biochemical Engineering it is common to use a capital $L$ for the volumetric unit liter and $\mathrm{mL}$ and $\mu \mathrm{L}$ for milliliter and microliter, respectively. This is also valid for publications in scientific journals.

$\mathrm{rpm}$ The unit rpm (revolutions per minute) instead of the SI unit $\mathrm{s}^{-1}$ is commonly used for the stirrer speed in biological stirred tank reactors and for the shaking frequency of shake flask cultures. 


\subsection{Materials and Methods}

\subsubsection{Equipment and Consumables}

Table 7-1: Equipment for general purposes

\begin{tabular}{|c|c|}
\hline Equipment & Supplier \\
\hline Shaking incubator Multitron & Infors, Bottmingen \\
\hline Autoclave Systec 5075 ELV & Systec, Wettenberg \\
\hline Photometer Spectronic 1001 & Bausch \& Lomb, \\
\hline Photometer Spectronic 501 & Bausch \& Lomb \\
\hline Photometer Genesys 20 & Thermo Spectronic, Erlangen \\
\hline pH meter CG853 & Schott, Mainz \\
\hline Bench-top centrifuge Mikro20 & Hettich, Tuttlingen \\
\hline Drying oven $\left(80^{\circ} \mathrm{C}\right)$ & Binder, Tuttlingen \\
\hline Incubator BD53 $\left(37^{\circ} \mathrm{C}\right)$ & Binder, Tuttlingen \\
\hline $\begin{array}{l}\text { HPLC } 1100 \text { series quarternary pump, standard autosam- } \\
\text { pler, and vacuum degaser }\end{array}$ & Agilent, Böblingen \\
\hline HPLC column GROM-SIL 100 ODS-2FE (250 x 5 mm) & Grom, Rottenburg-Hailfingen \\
\hline UV/VIS detector S3300 for HPLC & Sykam, Fürstenfeldbruck \\
\hline HPLC column oven S4110 & Sykam, Fürstenfeldbruck \\
\hline Mass flow controller MFC (Brooks 5850E) for oxygen flow & Westphal, Ottobrunn \\
\hline Mass flow controller MFC (Brooks 5850TR) for air flow & Westphal, Ottobrunn \\
\hline Mass flow control unit WMR4008 for up to eight MFCs & Westphal, Ottobrunn \\
\hline Microscope Axiolab & Zeiss, Göttingen \\
\hline Analytical balance Explorer E121245 (10 mg - $210 \mathrm{~g})$ & Ohaus, Gießen \\
\hline Analytical balance Explorer E1M213 (5 g - 32 kg) & Ohaus, Gießen \\
\hline Analytical balance AG $285(0,01 / 0,1 \mathrm{mg}-81 / 210 \mathrm{mg})$ & Mettler Toledo, Gießen \\
\hline $\begin{array}{l}\text { Micropipettes Pipetman }{ }^{\circledR} \text { P20, P200, P1000, P10mL for } \\
\text { variable volumes \#F123600, F123615, F123602, F161201 }\end{array}$ & Gilson, Bad Camberg \\
\hline Multichannel micropipette Pipetman ${ }^{\circledR} 8$ X200 \# & Gilson, Bad Camberg \\
\hline $\begin{array}{l}\text { Blood sugar detector AccuChek Comfort \#1 } 215961 \text { with } \\
\text { sensor sticks \#1 } 216156\end{array}$ & Roche Diagnostics, Mannheim \\
\hline
\end{tabular}




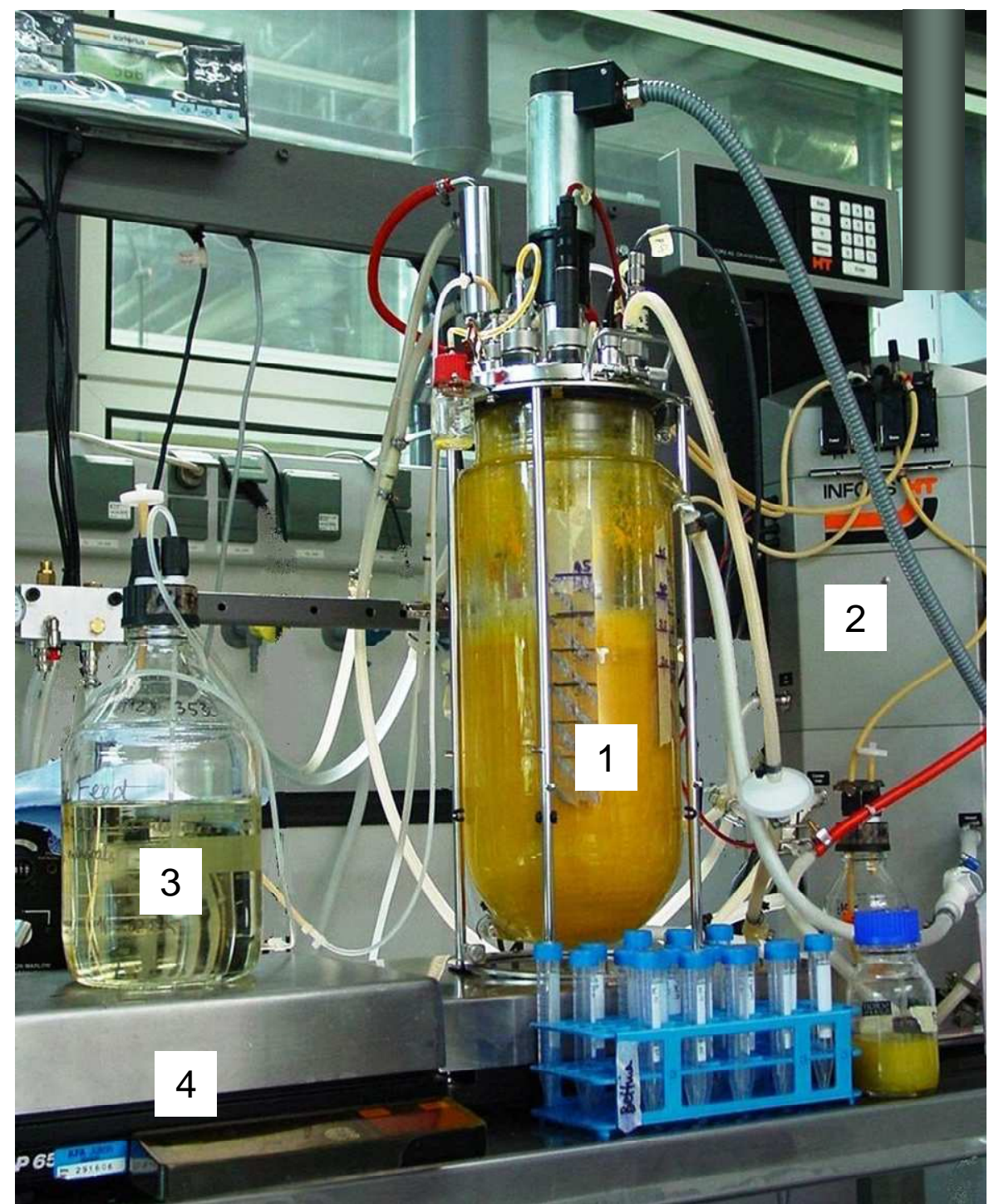

Figure 7-1: $\quad$ Setup of the Labfors ${ }^{\circledR}$ unit with a $7.5 \mathrm{~L}$ stirred tank reactor (1), placed on a balance, a control unit (2), and the feeding solution (3) on an extra balance (4) for the reference B2 processes with continuous feeding.

Table 7-2: Equipment for B2 processes at laboratory scale

\section{Equipment}

Supplier

Bench-top fermentor Labfors ${ }^{\circledR}$ with a $7.5 \mathrm{~L}$ jacketed glass vessel and Labfors ${ }^{\circledR}$ control unit (Figure 7-1); software: IRIS NT PRO Balance 4.11 (Infors, Bottmingen)

DO probe InPro 6000 \#52 200072

Mettler Toledo, Gießen

pH probe 405-DPAS-SC-K8S/325 \#10 4054482

Mettler Toledo, Gießen

$\mathrm{pH}$ probe SteamLine SL 80-325pH

Schott, Mainz

Extra balance Type I 6800 for feed

Sartorius, Uttingen

Flow control unit Type YFC 02 Z

Sartorius, Uttingen 
Exhaust Gas Analyzer Easy Line

Sample Gas Delivery Unit Advance SCC-F

Sample Gas Cooler Advance SCC-C

Peristaltic pump BVP Standard \#ISM444

with EasyLoad pump head \#MF0313

Peristaltic pump REGLO Analog MS-4/12 \#ISM796
ABB Germany, Mannheim

ABB Germany, Mannheim

ABB Germany, Mannheim

Ismatec, Wertheim-Mondfeld

Ismatec, Wertheim-Mondfeld

Table 7-3: Equipment for B2 processes at milliliter scale

\begin{tabular}{|c|c|}
\hline Equipment & Supplier \\
\hline $\begin{array}{l}\text { Laboratory robot GENESIS RSP } 150 \text { equipped with eight } \\
\text { teflon-coated low volume pipettes, wash station and } \\
\text { cooled carrier for } 3 \text { MTPs; software: GEMINI } 3.40 \text { SP } 1 \\
\text { (Tecan) with integrated routines programmed in LabVIEW } \\
6.1 \text { (National Instruments, München) }\end{array}$ & Tecan, Crailsheim \\
\hline $\begin{array}{l}\text { Reaction block for } 48 \text { parallel bioreactors (Figure 3-6) with } \\
\text { a cover lid for equal gas distribution into the vessels via } 48 \\
\text { hollow axles (Figure 7-2); software for magnetic drive: } \\
\text { Teleshake } 1.2(H+P \text {, Oberschleißheim) }\end{array}$ & $\mathrm{H}+\mathrm{P}$ Labortechnik, Oberschleißheim \\
\hline $\begin{array}{l}\text { Magnetic turbines (PEEK) with encapsulated magnets } \\
\text { (Figure 7-3) }\end{array}$ & Fermetronic, München \\
\hline Container for autoclaving the cover lid with stirrers & TUM (workshop BioVT) \\
\hline Milliliter bioreactors (PP) with four baffles (Figure 7-2) & $\mathrm{H}+\mathrm{P}$ Labortechnik, Oberschleißheim \\
\hline Photometric microplate reader FLUOstar Galaxy & BMG Labtech, Offenburg \\
\hline Columbus $^{\mathrm{TM}}$ microplate washer with $16 \mathrm{x}$ manifold & Tecan, Crailsheim \\
\hline Cooled carrier & Tecan, Crailsheim \\
\hline $6 x$ and $3 x$ MTP carrier for liquid reservoirs & TUM (workshop BioVT) \\
\hline Waterbath Haake W15 with temperature control unit C10 & Thermo Haake, Karlsruhe \\
\hline Temperature sensor 5790 NN (Schott \#285105254) & VWR International, Darmstadt \\
\hline $\begin{array}{l}\text { Miniature glass } p H \text { probe N6000A \#6626109 } \\
\text { (Schott \#285105151) }\end{array}$ & VWR International, Darmstadt \\
\hline $8 \times 2$ sensor block for $D O$ measurements (prototype) & PreSens, Regensburg \\
\hline $\begin{array}{l}\text { Dispensette }{ }^{\circledR} \text { III Typ Easy 2.5-25 mL (VWR Brand) } \\
\text { \#6125519 }\end{array}$ & VWR International, Darmstadt \\
\hline $\begin{array}{l}\text { Tool for incision of volumetric scores }(10-15 \mathrm{~mL}) \\
\text { (Figure 7-9) }\end{array}$ & TUM (workshop BioVT) \\
\hline
\end{tabular}




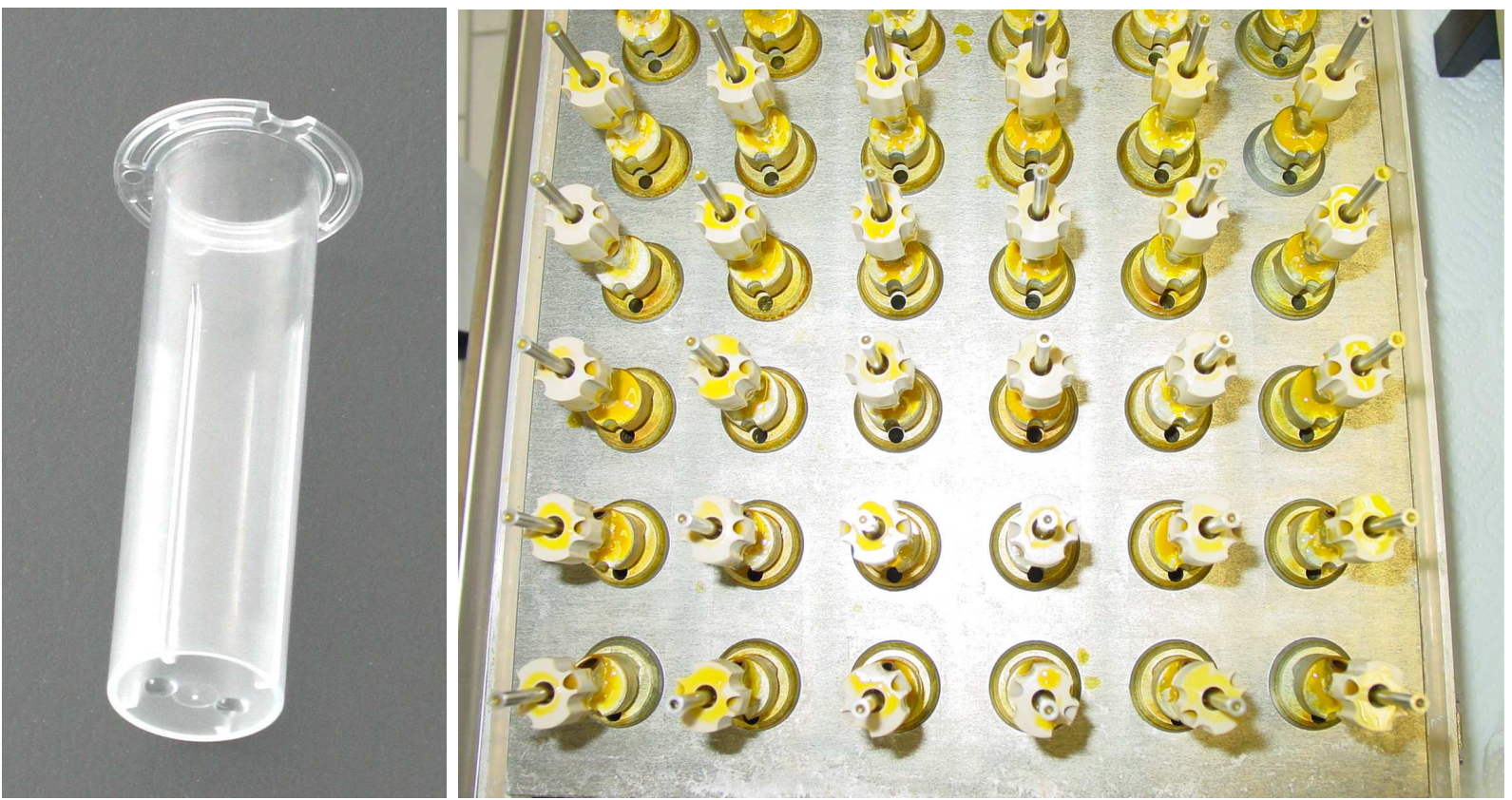

Figure 7-2: Left: Injection-molded polysterene (PS) milliliter bioreactor with four baffles and optical lenses at the bottom intended for internally immobilized fluorescence spots. Right: Cover lid of the reaction block with 48 gas-inducing impellers on hollows axles (photographed upside down after the completion of a 48 -fold B2 process).

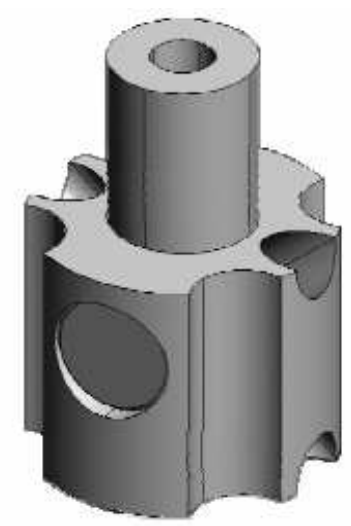

Figure 7-3: Gas-inducing turbine fabricated out of polyether ether ketone (PEEK) with two cavities for PEEK-encapsuled magnets on opposing sides, a central channel for the suction of gas into the liquid, and diagonal channels for expelling the mixed gas/liquid phase.

Table 7-4: Glassware and consumables

\begin{tabular}{ll}
\hline Consumables & Supplier \\
\hline HydroPlates ${ }^{\circledR}$ HP96L (Figure 7-4) \#B011022-32013 & PreSens, Regensburg \\
AccuChek Comfort sensor sticks \#1 216 156 & Roche Diagnostics, Mannheim \\
96 well black clear bottom plates, flat bottom \#3631 & Costar Corning, Wiesbaden \\
96-deep well MTPs U96 PP; $2 \mathrm{~mL}$ per well & Nunc, Wiesbaden \\
8-well reservoirs, 32 mL per deep well \#04.072.0380 & Nerbe Plus, Winsen/Luhe
\end{tabular}


Reagent reservoirs $(290 \mathrm{~mL}) \quad \# 04.072 .0320$

Perforated silicon covers for 96-well MTP \#04.093.0296

Eppendorf tubes 1.5 and $2 \mathrm{~mL} \# 211-2130$ and -2120

Reaction tubes (Falcon ) $15 \mathrm{~mL}, 50 \mathrm{~mL}$

Cryo-vials $2 \mathrm{~mL}$ \#E292.1

Disposable cuvettes 1.5 mL PMMA \#Y195.1

HPLC vials (amber) and lids \#5183-4493 and -4498

Graduated glass flasks (amber) $50 \mathrm{~mL}$ \#L456.2

Measuring glass cylinders $10 \mathrm{~mL}, 50 \mathrm{~mL}, 100 \mathrm{~mL}, 500 \mathrm{~mL}$

Baffled shake flasks $500 \mathrm{~mL}$ and $1000 \mathrm{~mL}$

Glass flasks (Schott) $100 \mathrm{~mL}, 250 \mathrm{~mL}, 500 \mathrm{~mL}, 1 \mathrm{~L}, 2 \mathrm{~L}$

Sterile filters (air in) Midisart ${ }^{\circledR} 2000$ PTFE $0.2 \mu \mathrm{m}$ (Sartorius) $\# 512-3250$

Sterile filters (air out) Sartobran 300 (Sartorius) \#5123333

Disposable autoclavable syringe filters (allround mem-

brane, $0.2 \mu \mathrm{m}$ pore size) \#P298.1

Filtered MultiGuard $^{\circledR}$ pipette tips (1-200 $\left.\mu \mathrm{L}\right) \quad \# 9771.1$

Filtered MultiGuard ${ }^{\circledR}$ pipette tips (100-1000 $\left.\mu \mathrm{L}\right)$ \#9773.1

Disposable pipette tips (various brands) $200 \mu \mathrm{l}, 1000 \mu \mathrm{L}$

Pump tubing Marprene ${ }^{\circledR}(\mathrm{ID}=1.6 \mathrm{~mm}$, wall thickness

$=1.6 \mathrm{~mm}$ ) for BVP pump (continuous feeding)

Pump tubing Pharmed ${ }^{\circledR}$ (ID $=0.89 \mathrm{~mm}$, wall thickness

$=0.85 \mathrm{~mm}$ ) for REGLO Analog pump MS-4/12 (intermittent feeding) \#SC0342
Nerbe Plus, Winsen/Luhe

Nerbe Plus, Winsen/Luhe

VWR International, Darmstadt

VWR International, Darmstadt

Carl Roth, Karlsruhe

Carl Roth, Karlsruhe

GGA, Moers

Carl Roth, Karlsruhe

Carl Roth, Karlsruhe

Schmitz Glasbläserei, München

Carl Roth, Karlsruhe

VWR International, Darmstadt

VWR International, Darmstadt

Carl Roth, Karlsruhe

Carl Roth, Karlsruhe

Carl Roth, Karlsruhe

Carl Roth, Karlsruhe

Watson-Marlow, Rommerskirchen

Ismatec, Wertheim-Mondfeld

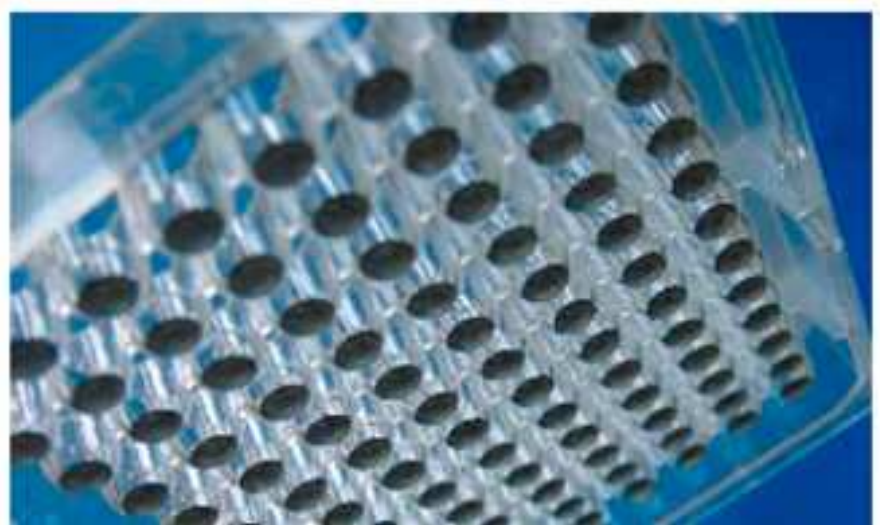

Figure 7-4: Bottom of a HydroPlate ${ }^{\circledR} \mathrm{HP} 96 \mathrm{~L}$ (PreSens, Regensburg) for parallel pH measurement in 96 wells with optical shielding. 


\subsubsection{Solutions and media}

\section{Growth media and feed solutions}

Feedstock or seed 1 cultures were carried out in complex media, containing yeast extract, veal infusion broth, glucose or sorbitol, respectively, $\mathrm{K}_{2} \mathrm{HPO}_{4}$, and chloramphenicol. In the medium for seed 2 cultures, veal infusion broth was replaced by salts and minerals. The composition of the batch medium for B2 cultivations is described by Zamboni et al. (2003). The feed solution contained minerals and $795 \mathrm{~g} \mathrm{~L}^{-1}$ glucose during lab-scale experiments. This solution was diluted 1:2 for cultivations at the milliliter scale. All solutions were sterilized by autoclaving.

Table 7-5: Composition of buffers and solutions

\begin{tabular}{|c|c|c|c|}
\hline Solution & Components & Order information & Quantity \\
\hline $0.4 \mathrm{M} \mathrm{NaP}_{\mathrm{i}}$ buffer $p H 7.4$ & $\mathrm{Na}_{2} \mathrm{HPO}_{4}$ & VWR, \#1.06586.0500 & $11.39 \mathrm{~g}$ \\
\hline \multirow{2}{*}{$\begin{array}{l}\text { (Adjust } p H \text { with } \mathrm{HCl} \text { or } \\
\mathrm{NaOH} \text {, if necessary.) }\end{array}$} & $\mathrm{NaH}_{2} \mathrm{PO}_{4} \cdot \mathrm{H}_{2} \mathrm{O}$ & VWR, \#1.06346.1000 & $2.73 \mathrm{~g}$ \\
\hline & Deionised water & & $1000 \mathrm{~mL}$ \\
\hline \multirow[t]{2}{*}{$1 \mathrm{M}$ sodium hydroxide } & $\mathrm{NaOH}$ & VWR, \#1.06482.1000 & $40.0 \mathrm{~g}$ \\
\hline & Deionised water & & $1000 \mathrm{~L}$ \\
\hline \multirow[t]{2}{*}{ 1.5 M citric acid } & $\mathrm{C}_{6} \mathrm{H}_{8} \mathrm{O}_{7} \cdot \mathrm{H}_{2} \mathrm{O}$ & Roth, \#5110.1 & $288 \mathrm{~g}$ \\
\hline & Deionised water & & $1000 \mathrm{~mL}$ \\
\hline \multirow[t]{2}{*}{$0.3 \mathrm{M}$ citric acid } & $\mathrm{C}_{6} \mathrm{H}_{8} \mathrm{O}_{7} \cdot \mathrm{H}_{2} \mathrm{O}$ & Roth, \#5110.1 & $57.6 \mathrm{~g}$ \\
\hline & Deionised water & & $1000 \mathrm{~mL}$ \\
\hline \multirow{4}{*}{$\begin{array}{l}200 \mathrm{mg} \mathrm{L}^{-1} \text { riboflavin } \\
\text { standard solution }\end{array}$} & Riboflavin & Sigma-Aldrich, \#R-4500 & $10 \mathrm{mg}$ \\
\hline & $1 \mathrm{M} \mathrm{NaOH}$ & see above & $200 \mu \mathrm{L}$ \\
\hline & $1.5 \mathrm{M}$ citric acid & see above & $1 \mathrm{~mL}$ \\
\hline & $0.4 \mathrm{M}$ buffer $p H 7.4$ & see above & $48.9 \mathrm{~mL}$ \\
\hline \multirow[t]{2}{*}{ Biospumex 30K (1:4) } & Biospumex $30 \mathrm{~K}$ & Cognis, France & $50 \mathrm{~g}$ \\
\hline & Deionised water & & $150 \mathrm{~mL}$ \\
\hline \multirow{2}{*}{$\begin{array}{l}12.5 \% \text { ammonium } \\
\text { hydroxide * }\end{array}$} & $\mathrm{NH}_{4} \mathrm{OH}(25 \%)$ & Roth, \#5460.2 & $200 \mathrm{~mL}$ \\
\hline & Deionised water & & $200 \mathrm{~mL}$ \\
\hline \multirow{2}{*}{$\begin{array}{l}\text { 200x chloramphenicol } \\
\text { stock solution ** }\end{array}$} & Chloramphenicol & Roche Diagnostics, \#634433 & $30.0 \mathrm{mg}$ \\
\hline & $\mathrm{EtOH}$ & VWR, \#1.00983.5000 & $10.0 \mathrm{~mL}$ \\
\hline
\end{tabular}

* Sterile filtration before use in milliliter setup!

${ }^{*}$ Prepare freshly for each process! 
Table 7-6: Antifoam solutions used in the milliliter setup

\begin{tabular}{|c|c|c|}
\hline Abbreviation & Specification and supplier & Chemical basis \\
\hline AF 1 & $\begin{array}{l}\text { Antifoam BC 86/013; Basildon Chemicals, } \\
\text { Oxon, UK }\end{array}$ & $\begin{array}{l}\text { organic antifoam compounds with a } \\
\text { small percentage of silicone }\end{array}$ \\
\hline AF 2 & 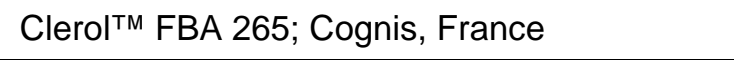 & alkylpolyalkoxyether, non ionic \\
\hline AF 3 & Biospumex ${ }^{\mathrm{TM}} 153 \mathrm{~K}$; Cognis, France & modified polyalkoxyether, non ionic \\
\hline AF 4 & Biospumex ${ }^{\mathrm{TM}} 30 \mathrm{~K}$; Cognis, France & modified polyalkoxyether, non ionic \\
\hline
\end{tabular}

\subsubsection{Bacterial strain}

The Bacillus subtilis strain used throughout this project was Bacillus subtilis RB50::[pRF69]n (Perkins et al., 1999). It is characterized by an enhanced purine synthesis pathway and a deregulated riboflavin biosynthesis. Its chromosome harbors multiple copies of the plasmid pRF69 which contains the rib operon with the four rib genes encoding the enzymes for the biosynthesis of riboflavin, and a chloramphenicol resistance gene for selection. In addition, a strong promoter replaces the native promoters of the operon for the constitutive transcription of the rib genes.

\subsubsection{Preparation of cell stocks and seed cultures}

\section{Cell stocks}

$49 \mathrm{~mL}$ of cell stock medium in a $500 \mathrm{~mL}$ baffled shake flask were inoculated with $1 \mathrm{~mL}$ of a defrosted $B$. subtilis stock vial. A few drops of antifoam AF1 were added. The culture was incubated at $150 \mathrm{rpm}$ and $37^{\circ} \mathrm{C}$ in the shaking incubator for approximately five to six hours until an optical density at $650 \mathrm{~nm} O D_{650}$ of 0.8 1.2 was reached. The culture was checked microscopically for contamination. Sterile glycerol (\#1.04091.1000, VWR International, Darmstadt) was added at $15 \%(\mathrm{v} / \mathrm{v})$ before filling $1.6 \mathrm{~mL}$ of the cell suspension into sterile cryo-vials or Eppendorf vials. The vials were stored at $-80^{\circ} \mathrm{C}$.

\section{Seed cultures}

The bacteria were grown in two consecutive seed cultures (seed 1 and seed 2) in designated media with chloramphenicol. $49 \mathrm{~mL}$ of seed 1 medium in a $500 \mathrm{~mL}$ baffled shake flask were inoculated with $1 \mathrm{~mL}$ of a defrosted $B$. subtilis cell stock vial. A few drops of antifoam AF1 were added. The culture was incubated at 150 $\mathrm{rpm}$ and $37^{\circ} \mathrm{C}$ in the shaking incubator for approximately five to six hours until 
$O D_{650}=0.8-1.2$ was reached. The seed 1 culture was diluted $1: 10^{5}$, and $500 \mu \mathrm{L}$ of this diluted suspension was added to $150 \mathrm{~mL}$ seed 2 medium in a $1000 \mathrm{~mL}$ baffled shake flask. A few drops of antifoam AF1 were added. Incubation was at $37^{\circ} \mathrm{C}$ and $180 \mathrm{rpm}$ for approximately $15-16$ hours until $O D_{650}=4-8$.

For inoculation of B2 processes at both laboratory and milliliter scale the seed 2 culture was diluted 1:20 into the batch medium, yielding an initial dry cell mass of approximately $0.1 \mathrm{~g} \mathrm{~L}^{-1}$.

\subsubsection{Cultivations at laboratory scale}

\section{Original fed-batch protocol (continuous reference operation)}

The reference riboflavin production processes with an initial volume of $3 \mathrm{~L}$ were carried out in a Labfors ${ }^{\circledR}$ unit (Infors, Bottmingen) including a balance, on which the $7.5 \mathrm{~L}$ stirred tank reactor was placed for monitoring the change of total weight during the feeding phase. The setup is displayed in Figure 7-1. It was equipped with a $\mathrm{pH}$ electrode, a probe for dissolved oxygen (DO), a sensor for foam detection for a closed loop-controlled antifoam addition, and with an exhaust gas analyzer for online monitoring of oxygen and carbon dioxide concentrations. Cultivations were carried out essentially as described by Zamboni et al. (2003) for industrial process conditions.

(Standard B2 processes at DSM Nutritional Products were carried out starting with $6 \mathrm{~L}$ initial volume and followed an equivalent protocol.)

Operation at ambient pressure

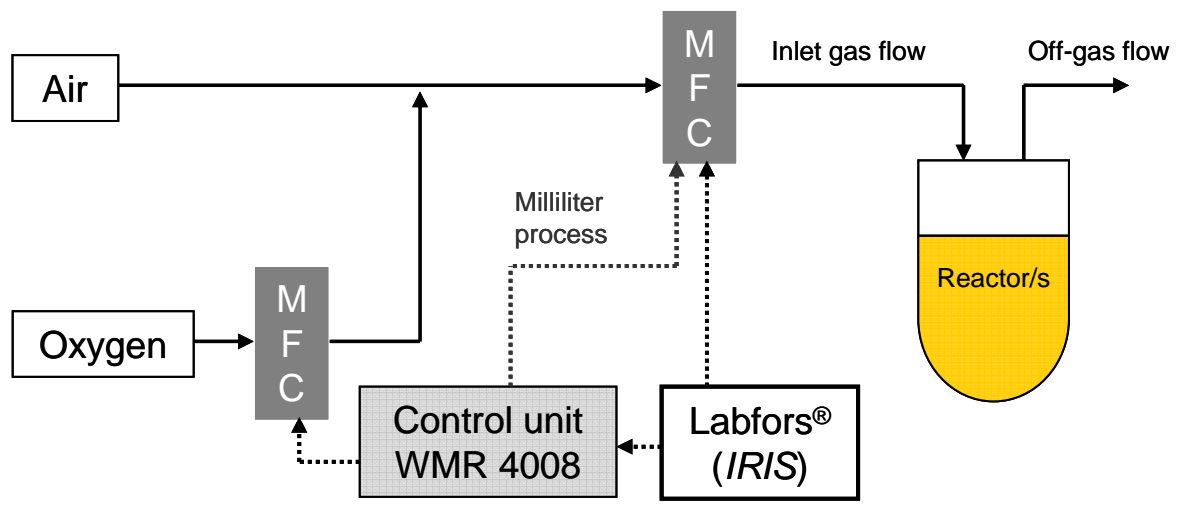

Figure 7-5: The oxygen flow and the total gas flow were controlle by two mass flow meters and a gas flow control unit (WMR 4008), indicated by the dotted lines. The control unit was operated either manually (as part of the milliliter setup) or was controlled by IRIS the Labfors ${ }^{\circledR}$ software. 
Oxygen was added to the inlet gas flow when ambient conditions were chosen instead of applying excess pressure (for details regarding the employed composition of the gas flow, refer to 4.3.2 B2 processes at ambient pressure with oxygen addition). The oxygen flow and the total gas flow (air plus oxygen) were regulated by two mass flow meters and a mass flow control unit (WMR 4008) according to the control scheme in Figure 7-5.

\section{Intermittent operation}

The setup for intermittent operation of the B2 process is displayed in the photograph in Figure 7-6. The stirrer speed was set to 1500 or 1200 rpm constantly. The pulsed addition of feed was realized via control sequences in the process software IRIS (Appendix 7.3.2) with a peristaltic pump (Reglo Analog). The pump dispensed the feed solution in pulses of less than ten seconds and provided the amount of substrate solution which otherwise would have been added continuously over the full length of an intermittent interval. Thus the total amount of added feed was the same in all cases.

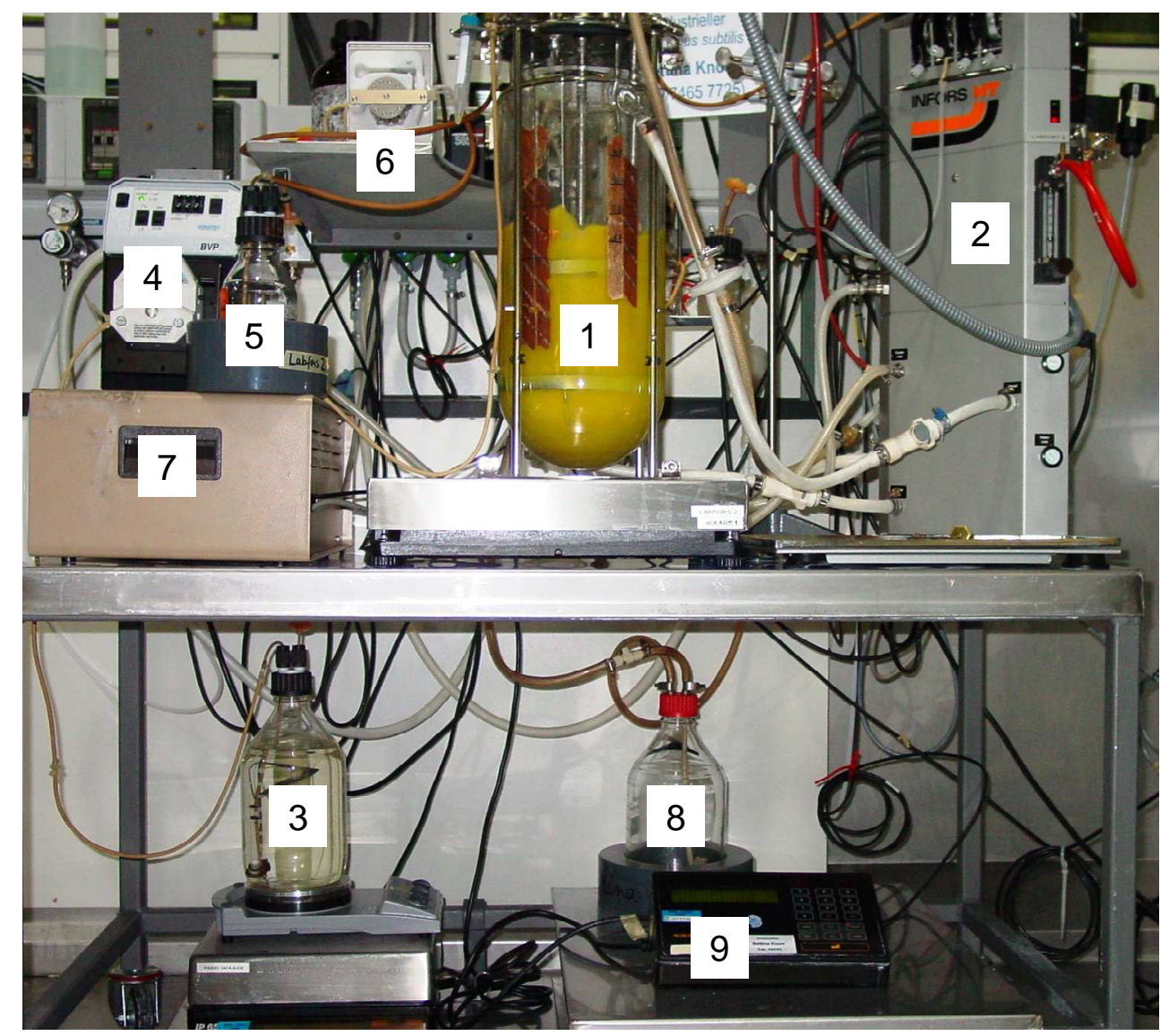

Figure 7-6: Setup of the Labfors ${ }^{\circledR}$ unit for intermittent operation of the $B 2$ process with a $7.5 \mathrm{~L}$ stirred tank reactor (1), placed on a balance, feeding solution (3) on an extra balance with a flow control unit (9) for communication with the Labfors ${ }^{\circledR}$ control unit (2), a feed pump (4), base solution (5) and an external base pump (6), a gas flow control unit in a protective housing (7), and a "foam trap" (8). 


\subsubsection{Cultivations in the automated milliliter setup}

Figure 7-7 displays the milliliter setup along with all the hardware components used for the automated operation of parallel B2 processes. This included a reaction block for up to 48 parallel milliliter stirred tank bioreactors, schematically displayed in Figure 7-8, a photometric microplate reader, a microplate washer for the recycling of the analytical plates, and a laboratory robot (Genesis RSP 150).

The laboratory robot was equipped with a liquid handling arm with eight parallel low volume pipettes that could be controlled individually, as well as with a robotic manipulator for the movement of microtiter plates within the area of the robot's worktable. Two carriers were installed on the worktable for the accurate positioning of microtiter plates. One of them held a HydroPlate ${ }^{\circledR}$ for at-line $p H$ measurements and two microtiter plates for automated at-line analyses during the cultivations. The second carrier provided space for six liquid containers (deep well troughs) for additive solutions (feed, base, antifoam) and for fluids used for sample dilution. One of the slots on this carrier was available for a deep well plate, into which samples could be dispensed and stored until their further treatment for offline analyses.

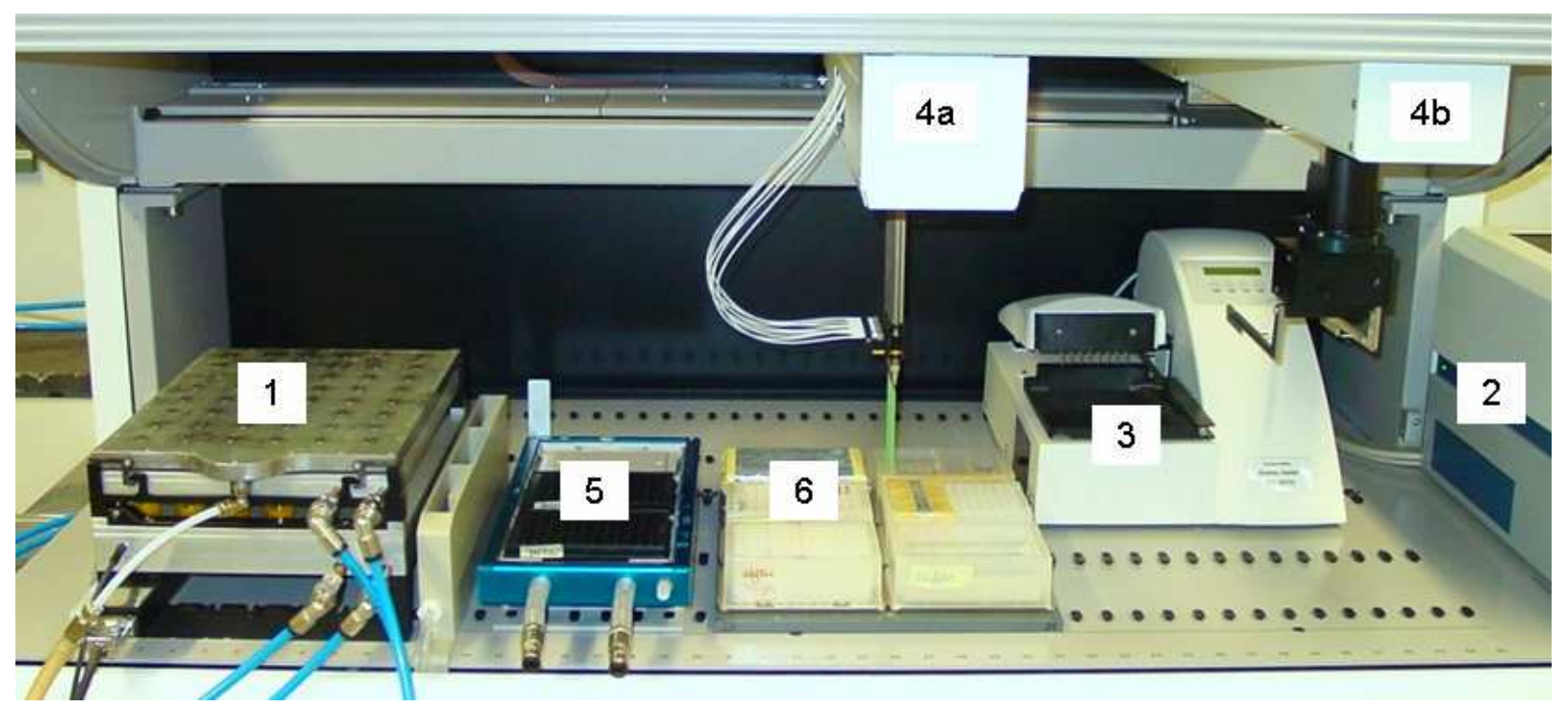

Figure 7-7: $\quad$ Automated screening setup at milliliter scale with a reaction block for up to 48 parallel stirred tank bioreactors (1), a photometric microtiter plate reader (2), a microtiter plate washer (3), and a laboratory robot, equipped with a liquid handling arm (4a) and a robotic manipulator (4b). Two carriers are installed on the worktable for the accurate positioning of microtiter plates. One carrier (5) holds a HydroPlate ${ }^{\circledR}$ and two microtiter plates for automated at-line analyses. The second carrier (6) gives space for six liquid containers (deep well troughs).

The completely automated process control was based on GEMINI, the laboratory robot software for liquid handling (Tecan, Crailsheim), which used embedded LabVIEW routines (National Instruments, München) for the communication of the ro- 
bot with the plate reader and plate washer ( 2 and 3 in Figure 7-7, respectively). Appendix 7.3.4 comprises a short description of the LabVIEW routines and GEMINI worklists integrated in the GEMINI script for the operation of 48 -fold parallel B2 processes.

Process control and monitoring during the 48 -fold parallel cultivations were carried out according to the scheme in Figure 4-37 in section 4.6.1 Scheduling the automated actions for 48 parallel B2 processes (page 90$)^{4}$. During eightfold parallel cultivations there was more time for liquid handling by the laboratory robot. Therefore monitoring was carried out every $30 \mathrm{~min}$ or $60 \mathrm{~min}$. The feed solution, containing $397 \mathrm{~g} \mathrm{~L}^{-1}$ glucose, was added in spikes of $35.6 \mu \mathrm{L}$ per reactor every 10 min during all processes, followed by the addition of base $\left(12.5 \% \mathrm{NH}_{4} \mathrm{OH}\right)$. The $p H$ was measured every $30 \mathrm{~min}$ and was used for the $p H$ control. The automated addition of antifoam solution (Biospumex $30 \mathrm{~K}$, diluted $1: 4$ ) every $30 \mathrm{~min}$ during 48-fold cultivations was started after six hours with $10 \mu \mathrm{L}$ per reactor per addition, which was decreased to $2 \mu \mathrm{L}$ per reactor per addition after 40 hours process time. During eightfold cultivations the antifoam addition was controlled manually.

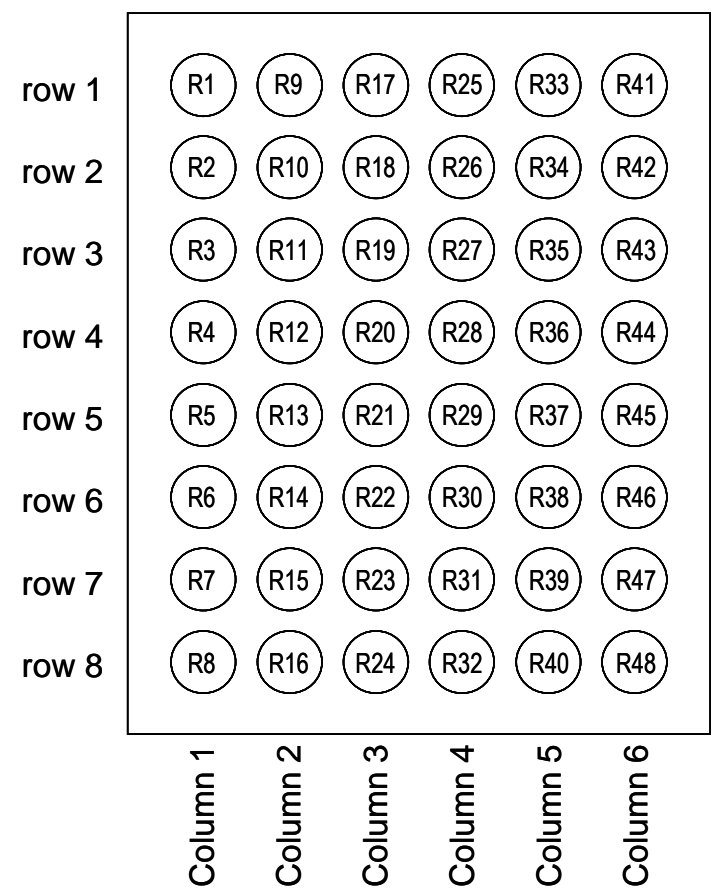

Figure 7-8: Schematic display of the reaction block for up to 48 parallel milliliter bioreactors (R1-R48) organized in six columns and eight rows. For a detailed description of the reaction block and the milliliter bioreactors refer to Puskeiler et al. (2005a).

\footnotetext{
${ }^{4}$ This schedule is displayed in greater detail in Figure 7-13 in Appendix 7.3.3.
} 
For the initiation of a process, a bottle-top dispenser (Dispensette) was used to add aliquots of $10.7 \mathrm{~mL}$ of inoculated batch medium to each of the parallel sterile milliliter reactors, which were already positioned in the open reaction block in a sterile hood. Next, the autoclaved cover lid with the magnetic impellers on the central hollow axles (photograph on the right in Figure 7-2) was inserted into the vessels and fastened with the clips designed for this purpose. After the arrangement of the complete unit on the worktable of the laboratory robot, the gas flow was started (total flow $=4.8 \mathrm{~L} \mathrm{~min}^{-1}$ ) and the stirrer speed was set to $2700 \mathrm{rpm}$ for the whole process. The temperature in the reaction vessels was kept at $39{ }^{\circ} \mathrm{C}$. The temperature for headspace cooling was $4{ }^{\circ} \mathrm{C}$ during the first eight hours of the process in order to initially suppress as much evaporation as possible. It was then raised to values between $15^{\circ} \mathrm{C}$ and $30^{\circ} \mathrm{C}$ in order to achieve similar volumetric increase compared to the reference process at laboratory scale.

During the processes the volume in the eight reactors of the first column was monitored according to externally incised volumetric scores (Figure 7-9). The levels were according to known volumes of medium stirred at the same speed and temperature, aerated with the same gas flow as during the actual B2 processes.
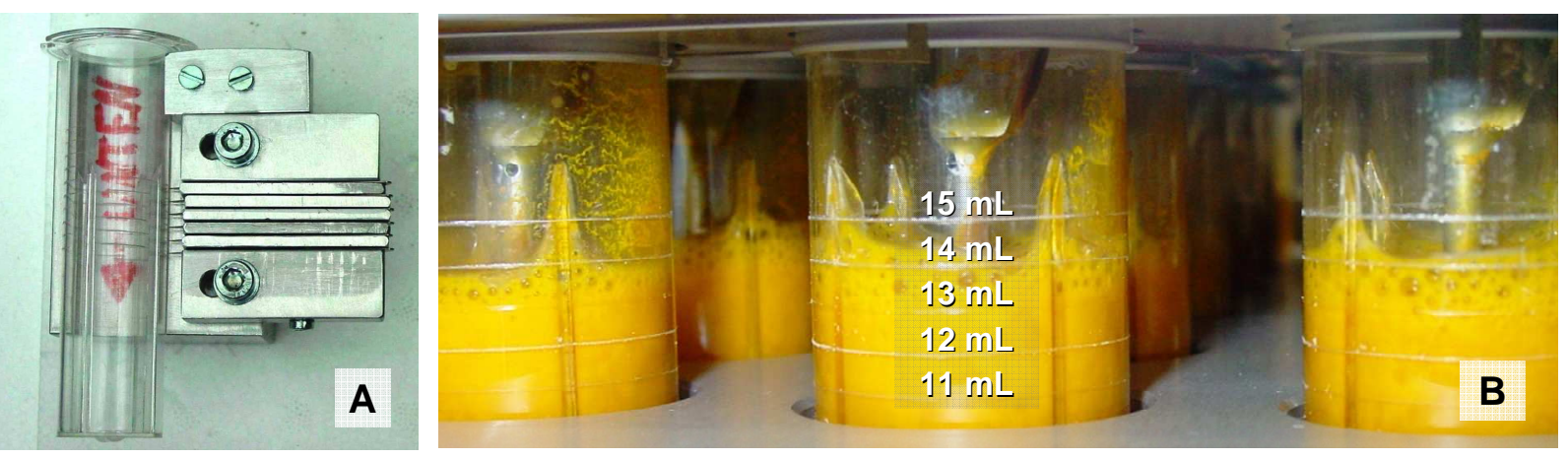

Figure 7-9: A: Tool for the incision of external volumetric scores into the walls of milliliter bioreactors. B: Graduated milliliter reactors in the first column of the reaction block. The position of the incised scores is based on known volumes of medium stirred at the same speed and temperature, and aerated with the same gas flow as during the actual B2 processes.

At the end of every parallel cultivation the final reactor volumes and the dry cell mass in each of the reactors were estimated gravimetrically. The final riboflavin concentration was determined by means of HPLC and absorbance.

\subsubsection{Gas input and measurement of dissolved oxygen in milliliter bioreactors}

The gas flow for all milliliter vessels $\left(4.8 \mathrm{~L} \mathrm{~min}^{-1}\right)$ was provided to the reaction block through a sterile air filter into one central inlet of the cover lid, was evenly spread inside the lid, and was guided individually into the headspace of each reactor with $100 \mathrm{~mL} \mathrm{~min}^{-1}$. From there a fraction of it was sucked through two oppos- 
ing holes in the upper part of each central hollow axle, triggered by the gasinducing character of the turbines, and was dispersed into the liquid phase. The exhaust gas escaped each reactor to the surrounding environment through an individual channel in the cover lid, which was also the access duct for the robotic needles.

The addition of oxygen to the inlet gas flow was realized with the same setup of mass flow controllers plus control unit that was used for the experiments at laboratory scale (Figure 7-5). The increase of the oxygen fraction in the gas flow was only roughly according to the previously established profile (section 4.3.2 B2 processes at ambient pressure with oxygen addition). It was mainly based on the actual signals of dissolved oxygen. The goal was to avoid minimum dissolved oxygen values $<5 \%$ and to prevent unnecessarily high maximum dissolved oxygen values at the same time. Hence, the increase of oxygen addition was sometimes later compared to the original profile. The oxygen fraction in the gas flow was raised from $21 \%$ (air without extra oxygen) to $35 \%$ after five to six hours, to $50 \%$ after eight to ten hours, to $55 \%$ after $12-15$ hours, and to 57-60 \% around 30-32 hours until the end of the process.
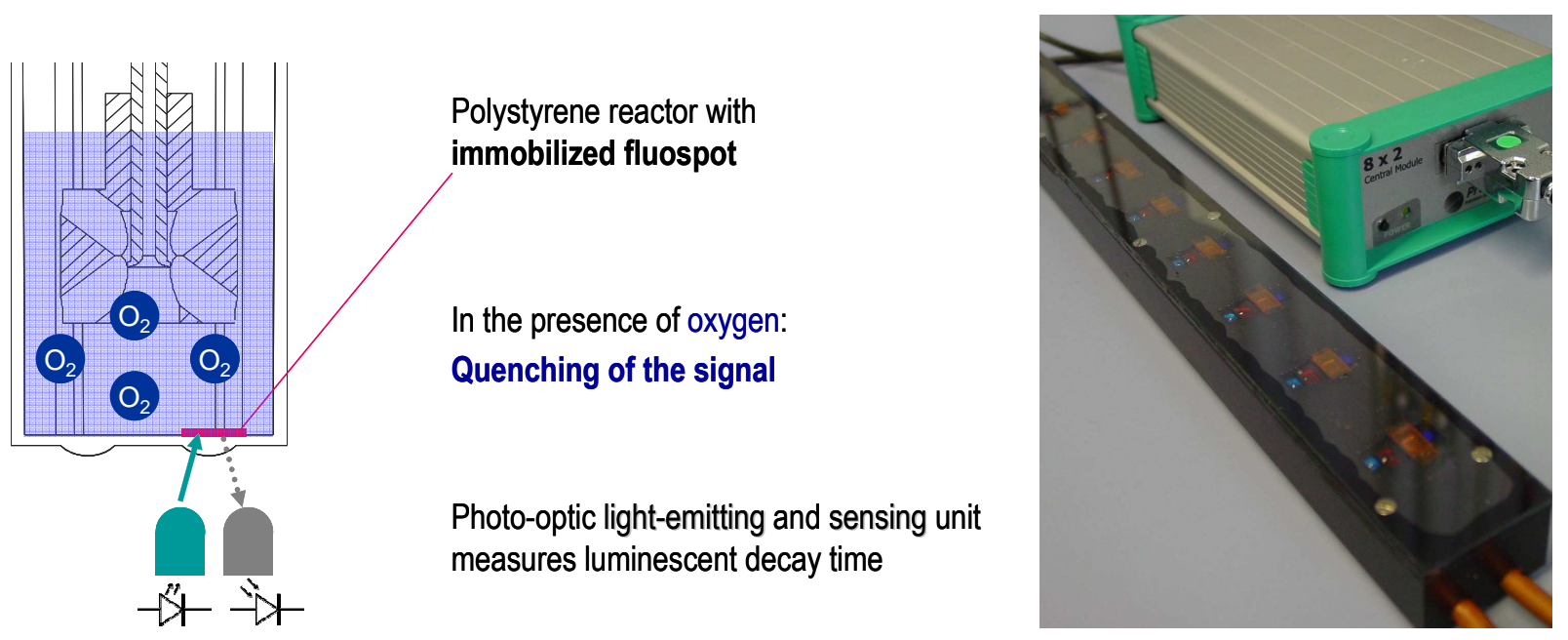

Figure 7-10: Principle of and hardware for the measurement of dissolved oxygen in milliliter bioreactors with internally immobilized fluorescence spots and an $8 \times 2$ external photo-optic sensor unit (PreSens, Regensburg).

Dissolved oxygen was monitored in the first column of the milliliter setup (eight parallel reactors) with an 8-fold sensor unit prototype (PreSens, Regensburg). The measurement was based on the oxygen-dependent luminescent decay time of a fluorophore in sensor spots immobilized internally at the bottom of the respective milliliter bioreactors. The signal could be detected from the outside, as indicated in Figure 7-10, and was converted to dissolved oxygen values by a LabVIEW routine. The $8 \times 2$ sensor unit had to be referenced according to the manufacturer's 
guidelines. Additionally, a calibration of the senor unit was carried out at the beginning of every B2 cultivation in the milliliter setup. The initial dissolved oxygen right after inoculation under regular operation conditions was designated $100 \%$. Next, the impellers were stopped for five to ten minutes to yield the $0 \%$ values.

\subsubsection{Parallel $\mathrm{pH}$ measurement and control at the milliliter scale}

Commercially available HydroPlates ${ }^{\circledR}$ HP96L (PreSens, Regensburg) were used for the parallel at-line measurement of $\mathrm{pH}$ every $30 \mathrm{~min}$ in the photometric plate reader of the milliliter setup. HydroPlates ${ }^{\circledR}$ have integrated $\mathrm{pH}$-sensor layers immobilized at the bottom of each well of a 96-well round bottom microtiter plate (Figure 7-4). The sensor contains two different fluorescent dyes in a constant ratio. One dye is sensitive to $\mathrm{pH}$, the other one is independent of the $\mathrm{pH}$. The detected fluorescence intensities of each well are correlated by division to yield an internally referenced signal $I_{R}$. This reduces the deviation from well to well significantly.

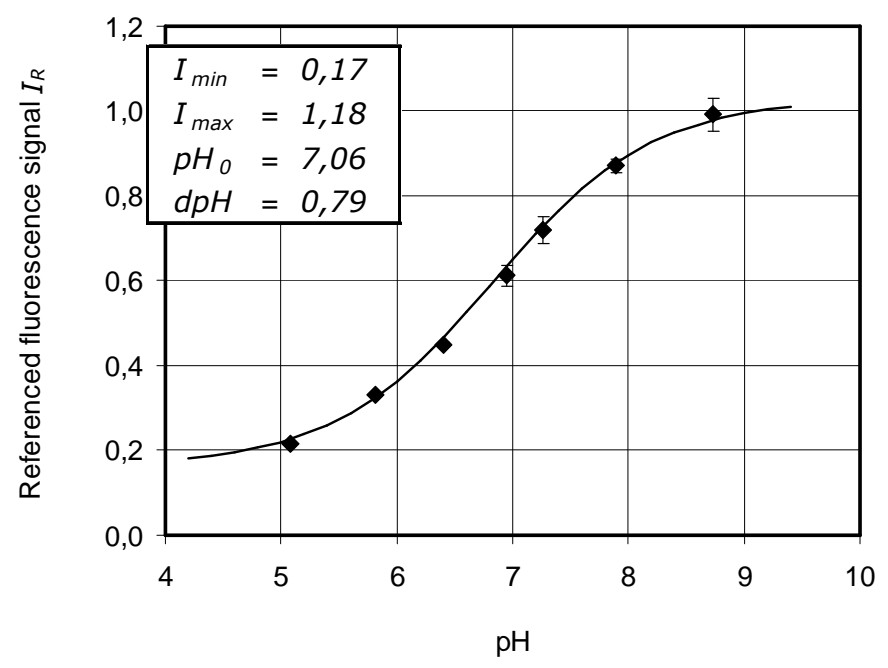

Figure 7-11: Typical $p H$ calibration curve in HydroPlate ${ }^{\circledR}$ HP96L using triplicate measurements of medium with different $\mathrm{pH}$ values $(10 \mu \mathrm{L}$ per well). The indicated constants are used for the calibration curve according to Equation 7-1.

Measuring the referenced intensity of medium with different $p H$ values or of appropriate buffers, which have an ionic strength equivalent to the expected samples, allows the establishment of a $\mathrm{pH}$ calibration curve. This curve is sigmoid and can be described by four constants, $d p H, p H_{0}, I_{\min }$ and $I_{\max }$, according to Equation 7-1 (PreSens, 2004).

Equation 7-1: $\quad \quad p H=\left(\ln \frac{I_{\min }-I_{\max }}{I_{R}-I_{\max }}-1\right) \cdot d p H+p H_{0}$ 
The $\mathrm{pH}$-dependent fluorescence was measured at $\lambda_{\text {excitation }}=485 \mathrm{~nm}, \lambda_{\text {emission }}$ $=520 \mathrm{~nm}$, the reference fluorescence at $\lambda_{\text {excitation }}=485, \lambda_{\text {emission }}=620 \mathrm{~nm}$. The evaluation of the data with the "solver" function (Microsoft excel) enables the determination of the four constants. A typical $\mathrm{pH}$ calibration curve, based on triplicate measurements with medium for the B2 process, is depicted in Figure 7-11. Similar curves for general applications are provided by the manufacturer along with specifications for HydroPlates ${ }^{\circledR}$ (PreSens, 2004).

\section{Control algorithm for an intermittent $\mathrm{pH}$ control}

A control algorithm with a proportional and a differential fraction (PD control) was developed for the calculation of the necessary volume of $12 \%$ ammonium hydroxide $\left(\mathrm{NH}_{4} \mathrm{OH}\right)$ to be added individually to each of the milliliter reactors, using the at-line $\mathrm{pH}$ values generated every $30 \mathrm{~min}$ and the base volume added during the previous 30 min interval (Equation 7-2). The control constants $K_{P}$ and $K_{D}{ }^{*}$ for the PD controller were determined empirically (Solf, 2003). They depended on the concentration of the $\mathrm{NH}_{4} \mathrm{OH}$ solution used for the adjustment, on the reactor volume, and on the intervals for base addition and $\mathrm{pH}$ detection. According to the individual output of the algorithm, equal amounts of base could be added every 10 min during the next 30 min interval.

Equation 7-2: $\quad V_{\text {base }}\left(t_{1}\right)=V_{\text {base }}\left(t_{0}\right)+K_{P}\left[\left(p H\left(t_{1}\right)-p H_{\text {set }}\right)+K_{D}{ }^{*}\left(p H\left(t_{1}\right)-p H\left(t_{0}\right)\right)\right]$
with
$\begin{array}{lll}V_{\text {base }}\left(t_{0}\right) & \text { base volume added during the previous } 30 \text { min interval } \\ & V_{\text {base }}\left(t_{1}\right) & \text { base volume to be added during the upcoming } 30 \text { min interval } \\ p H_{\text {set }} & p H \text { setpoint } \\ p H\left(t_{1}\right) & \text { actual } p H \text { value } \\ p H\left(t_{0}\right) & \text { previously measured } p H \text { value } \\ K_{P} & \text { empiric constant (proportional fraction) } \\ K_{D}{ }^{*} & \text { empiric constant (differential fraction) }\end{array}$

\subsubsection{Assessment of the dry cell mass concentration}

\section{Gravimetric determination}

The total dry mass TDM of samples was determined gravimetrically by drying $1 \mathrm{~mL}$ suspension in centrifuge cups at $80^{\circ} \mathrm{C}$ for at least 36 hours until weight constancy. The suspension contained cells as well as riboflavin crystals. For the dry cell mass $c_{X}$ the riboflavin concentration $c_{P}$ was subtracted from the total dry mass (Equation 7-3).

Equation 7-3: $\quad c_{X}=T D M-c_{P}$ 


\section{Estimation via optical density}

For more immediate growth monitoring the optical density at $650 \mathrm{~nm}$ in $1 \mathrm{~mL}$ of appropriately diluted offline samples was measured in a spectrophotometer. It was important to wait for constant $O D_{650}$ values which indicated the complete dissolution of riboflavin crystals. This could take several minutes for samples at elevated process times. For the estimation of the dry cell mass $c_{X}$, a linear correlation between $c_{X}$ and $O D_{650}$ was assumed (Equation 7-4).

Equation 7-4: $\quad c_{x}=0.33 \mathrm{gL}^{-1} \cdot O D_{650}$

\subsubsection{Quantification of riboflavin}

The riboflavin concentration $c_{P}$ in offline samples from B2 processes at both laboratory or milliliter scale were determined via absorbance or HPLC. The automated riboflavin detection in the milliliter setup was fluorimetrically.

\section{Absorbance}

For the quantification of the riboflavin concentration $C_{P}, 20 \mu \mathrm{L}$ process sample was diluted $1: 11$ in $1 \mathrm{M} \mathrm{NaOH}$ for dissolution of the riboflavin crystals. After one to 20 minutes $0.1 \mathrm{M}$ sodium phosphate buffer was added, yielding a respective dilution factor DF. The diluted samples were spun down for five minutes at $3300 \mathrm{~g}$ in a bench top centrifuge. The absorbance at $444 \mathrm{~nm}\left(A B S O_{444}\right)$ was determined in $1 \mathrm{~mL}$ of the cell-free supernatant, which allowed the calculation of the original riboflavin concentration according to Equation 7-5. For $A B S O_{444}>0.9$ the supernatants had to be further diluted.

Equation 7-5: $\quad C_{P}=D F \cdot A_{B S O} 44 \cdot 0.03305 \mathrm{~g} \mathrm{~L}^{-1}$

\section{Fluorescence}

The process samples were diluted according to the scheme in section 7.2.12. The measurement of the diluted riboflavin-containing samples in the 96-well plate took place in the photometric plate reader according to section 7.3.1.

HPLC

The product concentration was furthermore measured via HPLC (Agilent 1100 Series; column: GROM-SIL 100 ODS-2 FE $(250 \times 5 \mathrm{~mm}) ; 20 \%$ ethanol (compressibility $=60 \cdot 10^{-6}$ bar $^{-1}$ ); $50^{\circ} \mathrm{C}$; UV/VIS detection at $403 \mathrm{~nm}$ ). For sample preparation, $20 \mu \mathrm{L}$ process sample was added to $220 \mu \mathrm{L} 1 \mathrm{M} \mathrm{NaOH}$. After one to 20 minutes $180 \mu \mathrm{L} 1.5 \mathrm{M}$ citric acid was added for neutralization and $1400 \mu \mathrm{L}$ 
buffer for dilution. The samples were filtered into amber HPLC vials in order to prohibit light-induced degradation of riboflavin. Quantification of $C_{P}$ was according to a calibration curve based on riboflavin standard solutions.

\subsubsection{Glucose detection}

Glucose concentration in culture samples from B2 processes at laboratory scale were estimated offline with a blood sugar detector. This method had repeatedly shown satisfactory equivalence with a commonly used enzymatic assay that was based on the absorbance of generated NADH (data not shown). Automated glucose detection in the milliliter setup was fluorimetrically with the modified enzymatic assay described in section 4.4.3 Monitoring of the glucose concentration.

\section{Blood sugar detection with AccuChek ${ }^{\circledR}$}

AccuChek $^{\circledR}$ Comfort blood sugar detector \#1215961 Roche Diagnostics, Mannheim Accu-Chek ${ }^{\circledR}$ Sensor Comfort ( 1 x 50 sticks) \#1216156 Roche Diagnostics, Mannheim The application of AccuChek ${ }^{\circledR}$ sensor sticks is recommended in the range from $0.1 \mathrm{~g} \mathrm{~L}^{-1}$ to $6.0 \mathrm{~g} \mathrm{~L}^{-1}$ glucose. The process samples were diluted with phosphate buffer, if necessary. A sensor stick was slit into the respective slot in the detector. A drop of about $10 \mu \mathrm{L}$ of properly diluted sample was brought next to the perforated breach in the sensor stick to be sucked into the detection chamber solely by capillary forces (comparable to a drop of blood from a punctured finger).

The instrument calculates the concentration of glucose referring to an internal calibration curve, which is saved on a memory chip provided with each batch of sensor sticks, designated with a specific code number. Only sensor sticks belonging to batches with the same code number should be measured with the respective memory chip.

Modified enzymatic assay for fluorimetric detection of glucose in a 96-well plate Necessary active ingredients

Peroxidase (HRP)

Glucose oxidase (GOD)

10-Acetyl-3,7-Dihydroxyphenoxazin (ADHP)

or: Amplex ${ }^{\circledR}$ Red reagent (Molecular Probes)
\#127361 Roche Diagnostics, Mannheim

\#2208121 Roche Diagnostics, Mannheim

\#85500-5AS MoBiTec GmbH, Göttingen

\#A12222 MoBiTec GmbH, Göttingen 
Aliquoted stock solutions

ADHP, $20 \mathrm{mM}$ :

dilute the content of one vial ADHP (5 mg) in $1 \mathrm{~mL}$ dimethyl sulfoxide (DMSO; Carl Roth, Karslruhe) to yield approximately $20 \mathrm{mM}$ dye solution $\left(M W_{\mathrm{ADHP}}=257 \mathrm{~g} \mathrm{~mol}^{-1}\right)$; protect solution from light; freeze aliquots of $50 \mu \mathrm{L}$ at $-20^{\circ} \mathrm{C}$.

$\mathrm{HRP}, 200 \mathrm{U} \mathrm{mL}^{-1}$ : $\quad$ dilute the lyophillized enzyme in water according to the enzymatic activity stated on the package to yield $200 \mathrm{U} \mathrm{mL}^{-1}$; freeze aliquots of $100 \mu \mathrm{L}$ at $-20^{\circ} \mathrm{C}$.

GOD, $400 \mathrm{U} \mathrm{mL}^{-1}$ : dilute the lyophillized enzyme in water according to the enzymatic activity stated on the package to yield $400 \mathrm{U} \mathrm{mL}^{-1}$; freeze aliquots of $100 \mu \mathrm{L}$ at $-20^{\circ} \mathrm{C}$.

Reactive enzymatic dye solution for 48 wells ( $5 \mathrm{~mL})$ :

\begin{tabular}{|l|l|}
\hline ADHP $(20 \mathrm{mM})$ & $25 \mu \mathrm{L}$ \\
\hline $\operatorname{HRP}\left(200 \mathrm{U} \mathrm{mL}^{-1}\right)$ & $50 \mu \mathrm{L}$ \\
\hline GOD $\left(400 \mathrm{U} \mathrm{mL}^{-1}\right)$ & $100 \mu \mathrm{L}$ \\
\hline $\begin{array}{l}\text { Buffer }(0.1 \mathrm{M} \mathrm{NaPi}, p H=7.4) \\
\text { prepared according to section } 7.2 .2\end{array}$ & $4825 \mu \mathrm{L}$ \\
\hline
\end{tabular}

Store solution on ice! Freeze residual aliquot of expensive ADHP solution!

Preparation of samples was according to section 7.2.12. The measurement of the diluted glucose-containing samples in 96-well plates took place in the photometric plate reader according to section 7.3.1.

During the first measuring cycle $100 \mu \mathrm{L}$ of the reactive solution was added to each of the 48 well, which were identified in a respective layout in the software of the plate reader. The complete addition to all wells took 90 seconds. The six subsequent measuring cycles lasted 30 seconds each. The increase of fluorescence from the second until the fifth cycle was used for the determination of the equimolar conversion rate of glucose. The values measured during the addition of the dye solution in the initial cycle were not considered for the slope. The software provided with the photometric reader facilitated the evaluation by calculating the slopes per second from the consecutive fluorescence data for each well. A calibration curve based on previously determined glucose standards allowed the estimation of the respective glucose concentration. 


\subsubsection{Liquid Handling by the laboratory robot}

The laboratory robot can dispense liquids with a "single-pipetting" or a "multipipetting" mode. The "single-pipetting" mode allows aspiration of a given volume of a fluid and consecutive dispensing of the same volume. The "multi-pipetting" mode saves time by aspirating the summarized amount of several volumes of the same fluid to be added to different locations and then dispensing them separately.

Additionally, the robot contains a feature that enables individual settings for handling liquids with different fluid properties. This allows for example the specific adjustment of the position and speed for aspiration and dispensing, and optional pipetting delay times. These settings are stored for every fluid in liquid classes. Within each liquid class, the process software provides the ability to enhance the accuracy of the pipetting performance via an individual calibrating function for certain volumetric ranges, based on linear regression.

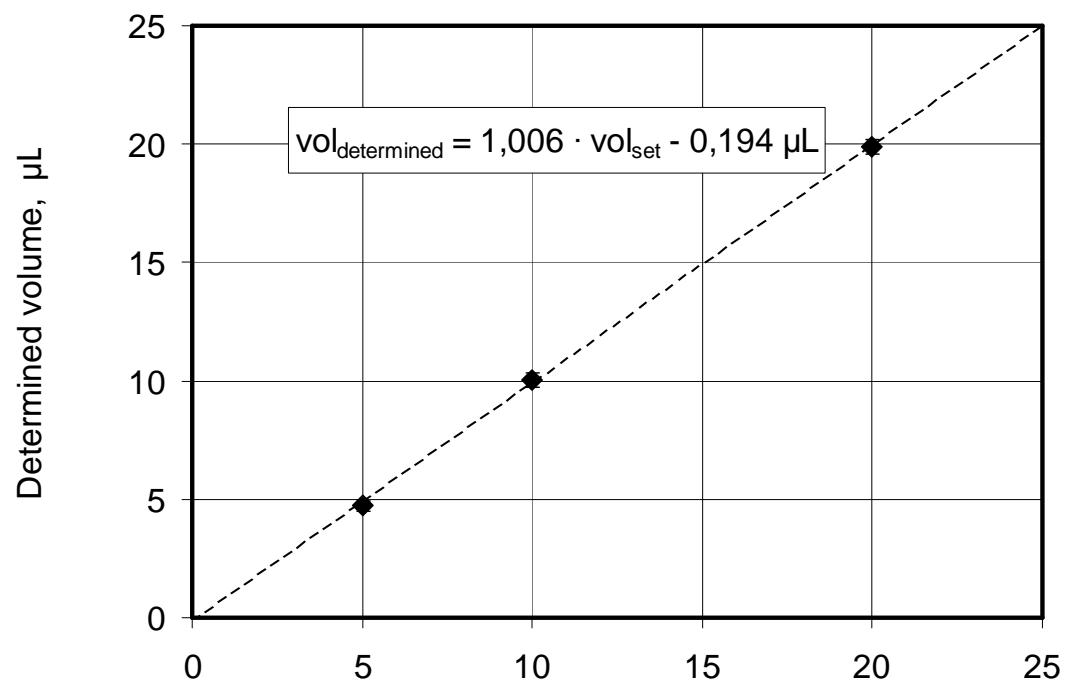

Set volume, $\mu \mathrm{L}$

Figure 7-12: Gravimetric test of a liquid class for pipetting undiluted process samples with the laboratory robot after optimization of this liquid class, using fresh samples from B2 processes at laboratory scale. Each symbol represents the average of ten pipetting events with three different needles. The framed equation reflects the linear regression (dashed line).

Liquid classes were established for every fluid handled by the laboratory robot during the B2 process at milliliter scale, particularly for the sample preparation. A detailed compilation of the liquid classes can be found in Appendix 7.3.5. Fluids with properties very similar to water were easy to handle and did not require much adjustment compared to the provided default values. The process samples from the B2 process, however, did not only contain a high cell concentration of up to $30-50 \mathrm{~g} \mathrm{~L}^{-1}$, but also needle-shaped riboflavin crystals, which made the accurate pipetting of this suspension a challenging task. Therefore, very slow pipetting 
and a prolonged delay time after aspiration were chosen for the respective liquid class, as this had been observed to work best during manual pipetting with micropipettes. The performance of this liquid class for handling $5 \mu \mathrm{L}, 10 \mu \mathrm{L}$, or $20 \mu \mathrm{L}$ of undiluted process samples, resulting from repeated optimization, is depicted in Figure 7-12. The sample volume used for the at-line analysis was $20 \mu \mathrm{L}$.

As the determination of riboflavin took place after the fourth dilution step of the "all-in-one" analysis, each of these steps had to meet very high standards with respect to precision and accuracy for the final dilution to yield reliable concentrations. The optimization of the liquid classes was performed gravimetrically and aimed at deviations from the setpoint $<3 \%$ and variations $<5 \%$ for sampling and sample dilution. In addition to relatively slow pipetting and dispensing actions for better accuracy, this required also the use of the "pinch-off valve" for enhanced precision. It was important for an optimal pipetting performance that the used low-volume pipettes of the robot were not damaged, particularly not on the external teflon coating at the tip of the pipettes.

Table 7-7: $\quad$ Performance of the liquid classes for the automated B2 process, assessed gravimetrically and expressed by the mean deviation from the relevant set volumes (accuracy) and the standard deviation around these volumes (precision).

\begin{tabular}{|c|c|c|c|c|c|}
\hline $\begin{array}{l}\text { Name of } \\
\text { liquid class }\end{array}$ & Fluids & Mode & $\begin{array}{c}\text { Volume } \\
\qquad \mathrm{L}\end{array}$ & $\begin{array}{c}\text { Accuracy } \\
\%\end{array}$ & $\begin{array}{c}\text { Precision } \\
\%\end{array}$ \\
\hline BK_sampling & sample (undiluted) & single & 20 & $<1$ & $<2$ \\
\hline BK_VD1 & $1 \mathrm{x}$ diluted sample & single & 10 & $<2$ & $<2$ \\
\hline \multirow{2}{*}{ BK_buffer_prep } & \multirow{2}{*}{$\begin{array}{l}\text { Aquatic solutions (buffer, } \mathrm{NaOH} \text {, } \\
\text { citric acid, } 2-3 x \text { diluted sample) }\end{array}$} & single & 100 & $<1$ & $<2$ \\
\hline & & single & 200 & $<1$ & $<2$ \\
\hline \multirow{2}{*}{ BK_feed_50\% } & \multirow{2}{*}{ Feed ( $397 \mathrm{~g} \mathrm{~L}^{-1}$ glucose) } & multi & 30 & $<1$ & $<2$ \\
\hline & & multi & 40 & $<1$ & $<2$ \\
\hline \multirow{2}{*}{ BK_NH4OH } & \multirow{2}{*}{$12.5 \% \mathrm{NH}_{4} \mathrm{OH}$} & multi & 2 & $<2$ & $<16$ \\
\hline & & multi & 20 & $<1$ & $<2$ \\
\hline \multirow{2}{*}{ BK_AF } & \multirow{2}{*}{$\begin{array}{l}\text { Antifoam Biospumex } 30 \mathrm{~K} \\
\text { (diluted 1:4 with water) }\end{array}$} & multi & 10 & $<3$ & $<7$ \\
\hline & & multi & 20 & $<3$ & $<4$ \\
\hline
\end{tabular}

The operation of the laboratory robot with the optimized liquid classes was examined gravimetrically for all the relevant fluids to be handled during sample preparations as well as for feed, base, and antifoam addition. The resulting performance of each liquid class is listed in Table 7-7 with the mean deviation from the 
relevant set volumes (accuracy) and the standard deviation around this volume (precision). Three to eight different needles of the laboratory robot were used for the assessment. Each of them pipetted the set volume ten times with the "singlepipetting" mode, or four times the six dispensing steps of a "multi-pipetting" cycle in the respective mode.

A consequence of the better liquid handling was the considerably slower operation compared to using the default settings of the liquid handling system. In order to save time, the "multi-pipetting" mode was employed wherever possible. The relatively high variance observed for pipetting low volumes of $\mathrm{NH}_{4} \mathrm{OH}$ was accepted, because the addition according to the control algorithm would quickly even out any possible deviation over the course of a cultivation. Despite low deviations of single pipetting steps, transfer volumes $<10 \mu \mathrm{L}$ were avoided for repeated dilution steps because of the accumulation of the errors.

\subsubsection{Dilution scheme for the automated "all-in-one" analysis}

The dilution of process samples during the automated B2 process in the milliliter setup took place in two microtiter plates (MTPs) which were divided into four sectors Q1-Q4 for 48 parallel samples each.
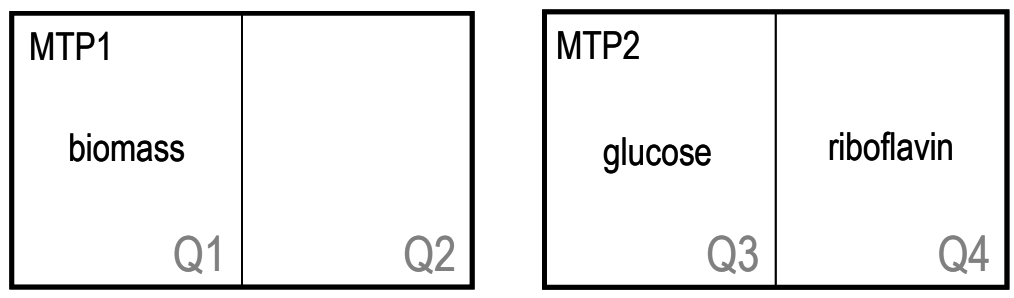

Q1: $220 \mu \mathrm{L} 1 \mathrm{M} \mathrm{NaOH}+20 \mu \mathrm{L}$ sample $+120 \mu \mathrm{L} 0.3 \mathrm{M}$ citric acid

$-10 \mu \mathrm{L}=350 \mu \mathrm{L}$

Q2: $230 \mu \mathrm{L}$ buffer $+10 \mu \mathrm{L}$ Q1

$-10 \mu \mathrm{L}=230 \mu \mathrm{L}$

Q3: $115 \mu \mathrm{L}$ buffer $+10 \mu \mathrm{L}$ Q2

$-25 \mu \mathrm{L}=100 \mu \mathrm{L}$

(plus $100 \mu \mathrm{L}$ reactive dye solution in the plate reader $=200 \mu \mathrm{L}$ )

Q4: $225 \mu \mathrm{L}$ buffer $+25 \mu \mathrm{L} \mathrm{Q3}$

$=250 \mu \mathrm{L}$ 


\subsection{Additional information}

7.3.1 Settings of the plate reader for $p H, O D$, glucose, and riboflavin detection The incubator of the photometric plate reader (FLUOstar Galaxy) was set to $30{ }^{\circ} \mathrm{C}$ for all measurements. The light ducts for signal detection were located underneath the microtiter plates (bottom). The light ducts for fluorescence excitation were also located at the bottom, while absorbance measurements were initiated through light ducts above the microtiter plates (top). The following settings were used for the analyses:

pH measurement in HydroPlates ${ }^{\circledR}$ HP96L

Position of light ducts: bottom/bottom

Reader configuration: Fluorescence Intensity and Time-Resolved Fluorescence

Plate mode

Basic parameters: $\quad$ Microplate: „Greiner 96“

No. of cycles: 1

No. of flashes: 10

Positioning delay: $0.2 \mathrm{~s}$

Number of multichromatics: 2

Excitation filters: 485 / 485

Emission filters: 520 / 620-10

Gain: 27 / 61

Calculation: Start:: 1

Stop: 1

Pause after cycle no.: 0

\section{Optical density}

Position of light ducts: top/bottom

Reader configuration: Absorbance

Plate mode

Basic parameters: $\quad$ Microplate: „Nunc Maxisorb 96“

No. of cycles: 2

No. of flashes: 20

Positioning delay: $0.2 \mathrm{~s}$

Number of multichromatics: 1

Excitation filter: $650 \mathrm{ND}$

Emission filter: empty

Gain: 30 (appropriate for all concentrations)

Calculation: Start:: 1

Stop: 2

Pause after cycle no.: 0 


\section{Fluorimetric determination of the riboflavin concentration}

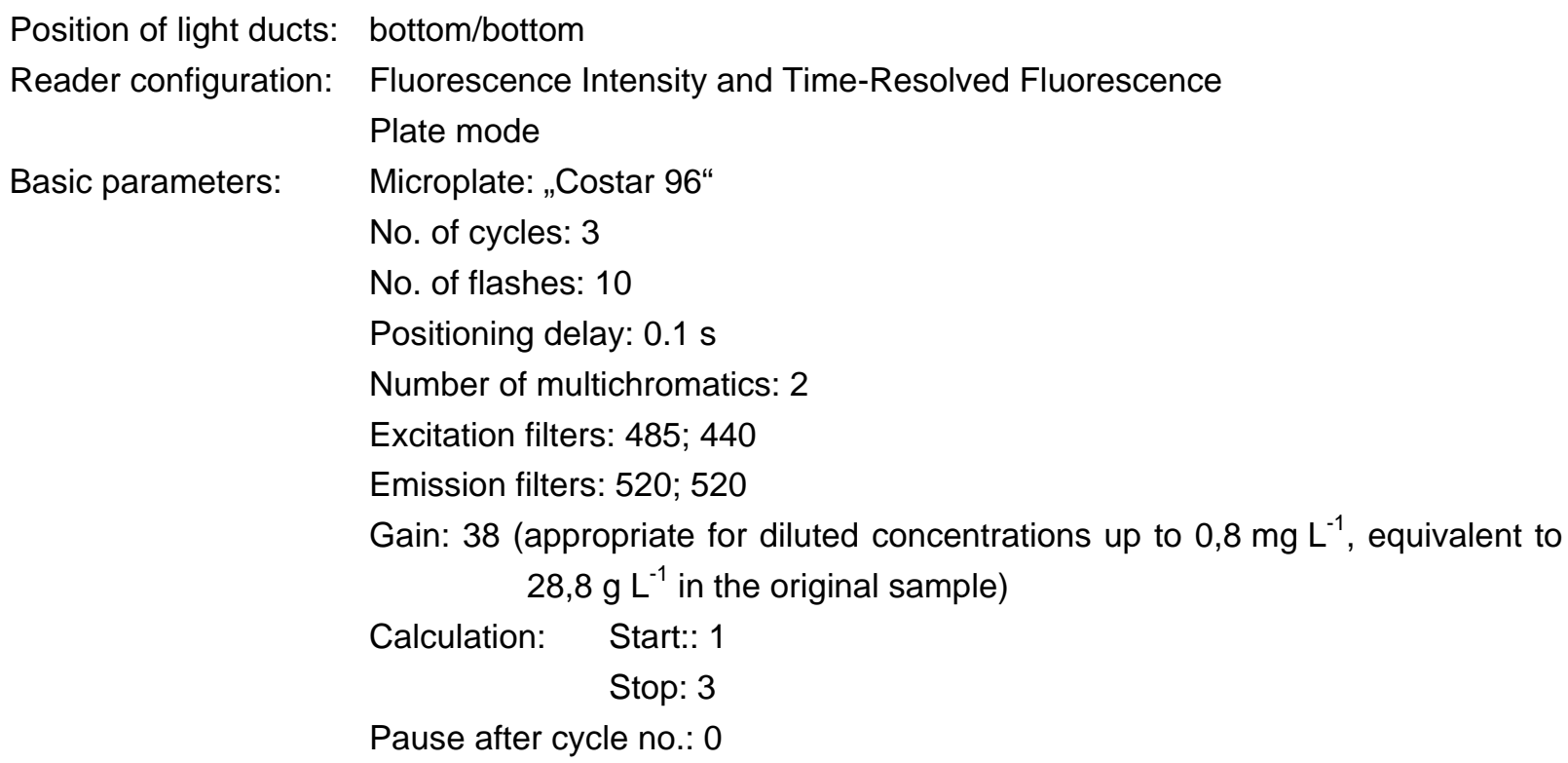

\section{Assessment of the glucose concentration}

Position of light ducts: bottom/bottom

Reader configuration: Fluorescence Intensity and Time-Resolved Fluorescence

Plate mode

Basic parameters: Microplate: „Costar 96“

No. of cycles: 7

No. of flashes: 10

Positioning delay: $0.1 \mathrm{~s}$

Cycle time: 30 (sufficient for 48 measurements in Q3)

Number of multichromatics: 1

Excitation filter: 544

Emission filter: 590

Gain: 22

Calculation: Start:: 2

Stop: 5

Pause after cycle no.: 0

Injection: $\quad$ First cycle $=$ preparation cycle $(100 \mu \mathrm{L}$ working solution to every well $)$

Pump settings: $\quad$ Pump Speed: $310 \mu \mathrm{L} \mathrm{s}^{-1}$

Invert Dispensing Function 


\subsubsection{IRIS scripts for intermittent operation at lab-scale}

The process software IRIS NT Pro Balance 4.11 (Infors, Bottmingen) allowed controlling certain parameters during processes in the Labfors ${ }^{\circledR}$ setup via scripts with simple numbered sequences. An active sequence was carried out periodically at a frequency which could be set in seconds after the sequence name in the first line of the sequence. Within a sequence the start of another sequence could be initiated, which ended the active sequence. The operation of various sequences did not have to be chronologically, but it was not possible to carry out several sequences at the same time. Within the scripts remarks could be added, indicated by "//" at the beginning of a line, which did not comprise any commands to be carried out. Process variables could be assessed as "values", indicated by the extension ".v", or as "setpoints", indicated with ".sp" after the name of the respective variable.

In the following, two complete IRIS scripts are given for either the intermittent operation of B2 processes with a 5 min feeding interval at excess pressure (no oxygen addition) or for B2 processes with oxygen addition at ambient pressure and with a 10 min interval for feeding and base control.

IRIS script for B2 processes with intermittent feeding every 5 min without oxygen addition

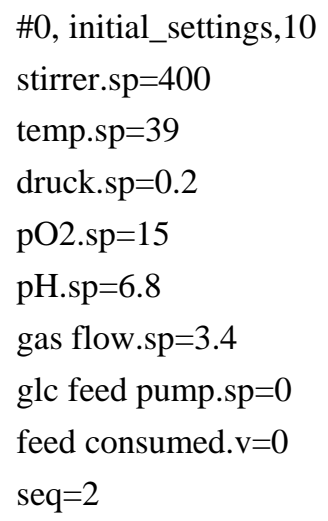


if $(\mathrm{pO} 2 . \mathrm{v}>70)$ and (seq_time>time $(3: 30))\{$ druck.sp=0.6; seq=2 $\}$ else $\{$ seq $=1\}$

\#2, start_pump, 1

// At the END of this cycle pump starts (after 1 second)

// and runs for the duration of the following sequence "feeding".

// Stirrer is set to a value close to max. stirrer speed

// in order to buffer the decrease in $\mathrm{pO} 2$ right after gluocse addition.

glc feed pump.sp $=51.918$

Act pmp speed.v=glc feed pump.sp

stirrer.sp $=1400$

wait_cycle.v $=0$

seq $=3$

\#3, feeding, 5

// The external feed pump is active for the duration of this cycle.

// This sequence lasts 5 seconds.

glc feed pump.sp $=0$

Act pmp speed.v=glc feed pump.sp

seq $=4$

\#4, complemetary_cycle,14

// This sequence allows easy calculation of the ( $\mathrm{t}$ cycle) when all the other sequences are 10sec.

// (Refer to sequence waiting_loop.)

// Then the duration for complementary_cycle=20sec-(feeding duration+1sec).

$/ /$ This sequence lasts $20 \mathrm{~s}-(1 \mathrm{~s}+$ duration of sequence \#3) $=14 \mathrm{~s}$

// In addition the value for consumed feed is updated.

feed consumed.v=((100.44-feed balance.v)/100*0.9947)*6000

seq $=5$

\#5, control_phase, 10

// In this sequence general control parameters during the feeding phase can be addressed.

// Attention: Parameters will be updated/adjusted only once every $20 \mathrm{sec!}$

// Stirrer speed is limited to a range between "safe" values in order to ensure high enough pO2.

feed consumed.v $=((100.44$-feed balance.v $) / 100 * 0.9947) * 6000$

if $(\mathrm{pO} 2 . \mathrm{v}<15)\{$ stirrer.sp=stirrer.sp+10*(pO2.sp-pO2.v) $\}$

if $(\mathrm{pO} 2 . \mathrm{v}>20)\{$ stirrer.sp=stirrer.sp+0.5*(pO2.sp-pO2.v) $\}$

if $($ stirrer.sp $<1200)\{$ stirrer.sp $=1200\}$

if (stirrer.sp>1500) \{stirrer.sp=1500\}

seq $=6$

\#6, waiting_loop, 10

// Duration of time between two actual feeding actions can be derived as follows:

$/ /(\mathrm{t}$ cycle $)=\left(\right.$ wait_cycle. $\left.v^{*} 20 \mathrm{sec}\right)+20$ sec.

Act pmp speed.v=glc feed pump.sp

wait_cycle.v=wait_cycle.v+1

if (wait_cycle.v $<14)\{$ seq $=5\}$ else $\{$ seq $=2\}$ 


\section{IRIS script for B2 processes with intermittent feeding and base control every 10 min and oxygen addition at ambient pressure}

// B2 Fbat with intermittent feeding every $10 \mathrm{~min}$ and intermittent base addition for $\mathrm{pH}$ control every $10 \mathrm{~min}$ // based on $\mathrm{pH}$ measurement every $30 \mathrm{~min}$, interlaced between the base additions by $15 \mathrm{~min}$.

// The necessary base volume to be added is calculated with a PID-like control algorithm.

// Oxygen content in air according to previous profiles:

// $21 \%$ until 3:30; 30\% until 5:30; 50\% until 7:30; 35\% until the end.

// Constant stirrer speed and air flow.

// The variables base_counter and pH_counter are increased by 1 every 20s.

// The variable batch_counter is increased by 1 only during the base addition, i.e. once every $10 \mathrm{~min}$.

$\# 0$, initial_settings, 10

stirrer.sp $=1200$

temp.sp=39

druck.sp $=0$

$\mathrm{pO} 2 . \mathrm{sp}=15$

gas flow. $\mathrm{sp}=3.4$

pH.sp $=6.8$

$\mathrm{pH} \_$value_0.v $=\mathrm{pH} . \mathrm{v}$

$\mathrm{pH} \_$value_1.v $=\mathrm{pH} . \mathrm{v}$

$\mathrm{pH} \_$counter.v $=0$

base_counter.v $=0$

base_vol_0.v $=0$

base_pump.sp=0

base_pump.bv=base_pump.sp

base consumed. $v=0$

batch_counter.v $=0$

glc feed pump.sp=0

glc feed pump.bv=glc feed pump.sp

feed consumed. $v=0$

MFC O2.sp=0

MFC O2.bv=MFC O2.sp

seq $=1$

\#1, base_vol_determination, 1

base_vol_1.v=base_vol_0.v+8*(pH.sp-pH_value_1.v)-6*(pH_value_1.v-pH_value_0.v)

seq $=2$

// Sequence 24 complements the 10 sec for Sequence 6 or $7+8$ or $27+28$

$/ /$ in combination with Sequence 5 and brings the base counter to 29 ( 0 to $29=30 * 20 \mathrm{sec}=10 \mathrm{~min}$ ).

\#24, complement_counter,10

base_counter.v=base_counter.v+1

$\mathrm{pH} \_$counter.v=pH_counter.v+1

seq $=2$ 
// Sequences 2-4 build one 20 sec-unit.

\#2, start_base_pump, 1

// At the END of this cycle the base pump starts (after 1 second)

// and runs for the duration of the following sequence "base_addition".

base_pump.sp=36.06*base_vol_1.v-1.815

IF (base_vol_1.v<=0.05) $\{$ base_pump.sp $=2.0\}$

IF (base_pump.sp>100) \{base_pump.sp=100\}

base_pump.bv=base_pump.sp

base consumed.v=base consumed.v+(base_pump.sp+1.815)/36.06

seq $=3$

\#3, base_addition, 10

// The external base pump is active for the duration of this sequence.

base_pump.sp=0

base_pump.bv=base_pump.sp

seq $=4$

\#4, compl_base_cycle2,9

// The duration for this sequence is

// $20 \mathrm{sec}$ - (duration"base_addition"+1sec).

base_counter.v $=0$

pH_counter.v=pH_counter.v+1

batch_counter.v=batch_counter.v+1

seq $=5$

// Sequences $5,7,8$ or $5+6$ or $5+31$ or $5,27,28$ are each one 20 sec-unit.

\#5, check_counters, 10

if $\left(\mathrm{pH} \_\right.$counter.v==45) $\{\mathrm{seq}=7\}$

if $\left(\left(\mathrm{pH} \_\right.\right.$counter.v<89) and $\left(\mathrm{pH} \_\right.$counter.v!=45) and (base_counter.v<28)) $\{$seq=6

if $\left(\left(\mathrm{pH} \_\right.\right.$counter.v<89) and $\left(\mathrm{pH} \_\right.$counter.v!=45) and (base_counter.v>=28)) $\{\mathrm{seq}=24\}$

if ( $\mathrm{pH} \_$counter.v $\left.>=89\right)\left\{\mathrm{pH} \_\right.$counter.v=0; seq=27\}

\#6, check_batch_conditions, 10

// Check if there is still glucose available after $22 * 10 \mathrm{~min}=220 \mathrm{~min}$ (=almost 4 hours).

// If not, pO2 rises above $120 \%$ and the "feeding phase" of the process begins with Sequence 10.

// Otherwise the process stays in base addition cycle of the batch phase.

// The setpoint of the oxygen flow is calculated based on $3 \mathrm{~L} / \mathrm{min}$ max flow of the MFC.

// Max. flow (100\% MFC) would be reached with a 5V signal (=50\% IRIS signal).

base_counter.v=base_counter.v+1

$\mathrm{pH} \_$counter.vepH_counter.v+1

if $(($ time $(3: 30)<=$ elapsed) AND (elapsed $<$ time $(5: 30)))\{$ MFC O2.sp=6.584 $\}$

$\mathrm{MFC} \mathrm{O} 2 . \mathrm{bv}=\mathrm{MFC} \mathrm{O} 2 . \mathrm{sp}$

O2flow_sp.v=(MFC O2.bv*2)/100*3

if $(($ batch_counter.v $>22)$ and $(p O 2 . v>100))\left\{p H \_v a l u e \_1 . v=p H . v ; s e q=10\right\}$ else $\{$ seq=5 
// Sequences 7-8 together with Sequenz 5 build one 20 sec-unit.

\#7, measure_pH,5

// Base volume adjustment is every $30 \mathrm{~min}$.

// The $\mathrm{pH}$ measurement, on which the adjustment is based,

$/ /$ is interlaced in between two adjustments also every $30 \mathrm{~min}$.

$/ /$ The actual $\mathrm{pH}$ value is updated in the following sequence 8 .

pH_value_0.v=pH_value_1.v

seq $=8$

\#8, pH_update,5

// The actual $\mathrm{pH}$ value is updated.

pH_value_1.v=pH.v

base_counter.v=base_counter.v+1

$\mathrm{pH} \_$counter.v=pH_counter.v+1

seq $=5$

// Sequences 27-28 together with Sequenz 5 build one 20 sec-unit.

\#27, base_vol_reset,5

// Base volume adjustment is every $30 \mathrm{~min}$ (at $\mathrm{pH} \_$counter $=0$ )

$/ /$ and is interlaced in between two $\mathrm{pH}$ measurements (at pH_counter=45).

base_vol_0.v=base_vol_1.v

IF (base_vol_1.v<0) \{base_vol_0.v=0 $\}$

seq $=29$

\#28, base_vol_determination,5

// New base volume determination.

base_vol_1.v=base_vol_0.v+8*(pH.sp-pH_value_1.v)-6*(pH_value_1.v-pH_value_0.v)

base_counter.v=base_counter.v+1

pH_counter.v=pH_counter.v+1

seq $=5$

// Seq10-22: Feeding phase with intermittent base control every 5 min and intermittent feed addtion every 10 min.

\#10, base_vol_determination, 1

base_vol_1.v=base_vol_0.v+8*(pH.sp-pH_value_1.v)-6*(pH_value_1.v-pH_value_0.v)

seq=11

// Sequenz 25 complements the 10sec for Sequenz 6 or $7+8$ or $27+28$ in combination with Sequenz 18

$/ /$ and brings the base counter to 29 ( 0 to $29=30 * 20 \mathrm{sec}=10 \mathrm{~min})$.

\#25, complement_counter, 10

base_counter.v=base_counter.v+1

pH_counter.v=pH_counter.v +1

seq $=11$ 
// Sequences 11-13 build one 20 sec-unit.

\#11, start_base_pump,1

// At the END of this cycle the base pump starts (after 1 second)

// and runs for the duration of the following sequence "base_addition".

base_pump.sp=36.06*base_vol_1.v-1.815

IF (base_vol_1.v<=0.05) \{base_pump.sp=2.0

IF (base_pump.sp>100) \{base_pump.sp=100\}

base_pump.bv=base_pump.sp

base consumed.v=base consumed.v+(base_pump.sp+1.815)/36.06

seq $=12$

\#12, base_addition, 10

// The external base pump is active for the duration of this sequence.

base_pump.sp=0

base_pump.bv=base_pump.sp

seq $=13$

\#13, compl_base_cycle,9

// The duration for this sequence is

// $30 \mathrm{sec}$ - (duration"base_addition"+1sec).

base_counter.v $=0$

$\mathrm{pH} \_$counter.v=pH_counter.v+1

seq $=14$

// Sequences 14-16 build one 20 sec-unit.

\#14, start_feed_pump,1

// At the END of this cycle pump starts (after $1 \mathrm{sec}$ ) and runs for the duration of the following sequence "feeding". glc feed pump.sp=42

glc feed pump.bv=glc feed pump.sp

seq $=15$

\#15, feeding, 14

// The external feed pump is active for the duration of this sequence.

// In addition the value for consumed feed is updated.

glc feed pump.sp $=0$

glc feed pump.bv=glc feed pump.sp

feed consumed.v $=((100.44$-feed balance.v $) / 100 * 0.9947) * 6000$

seq $=16$

\#16, compl_feed_cycle,5

// The duration for compl_feed_cycle=20sec-(feeding duration+1sec).

base_counter.v=base_counter.v+1

$\mathrm{pH} \_$counter.v=pH_counter.v+1

seq $=18$ 
// Sequence $18+20+21$ or $18+19$ or $18+32$ or $18+22+23$ each build one 20 sec-unit.

\#18, check_counters, 10

if ( $\mathrm{pH} \_$counter. $\left.\mathrm{v}==45\right)\{\mathrm{seq}=20\}$

if $\left(\left(\mathrm{pH} \_\right.\right.$counter.v<89) and $\left(\mathrm{pH} \_c o u n t e r . v !=45\right)$ and (base_counter.v<28) $\{$ seq=19\}

if $\left(\left(\mathrm{pH} \_\right.\right.$counter.v<89) and $\left(\mathrm{pH} \_\right.$counter.v!=45) and (base_counter.v $\left.\left.>=28\right)\right)\{$ seq=25

if (pH_counter.v>=89) \{pH_counter.v=0; seq=22\}

\#19, counting, 10

// The setpoint of the oxygen flow is calculated based on 3L/min max flow of the MFC.

// Max. flow (100\% MFC) would be reached with a 5V signal (=50\% IRIS signal).

base_counter.v=base_counter.v+1

$\mathrm{pH} \_$counter.v=pH_counter.v+1

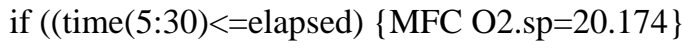

MFC O2.bv=MFC O2.sp

O2flow_sp.v=(MFC O2.bv*2)/100*3

seq $=18$

// Sequences 20-21 together with Sequenz 18 build one 20 sec-unit.

\#20, measure_pH,5

// Base volume adjustment is every $30 \mathrm{~min}$.

// The pH measurement, on which the adjustment is based,

$/ /$ is also every $30 \mathrm{~min}$, interlaced in between two adjustments.

// The actual $\mathrm{pH}$ value is updated in the following sequence 21.

$\mathrm{pH} \_$value_0.v=pH_value_1.v

seq $=21$

\#21, pH_update,5

// The actual $\mathrm{pH}$ value is updated.

$\mathrm{pH} \_$value_1.v=pH.v

base_counter.v=base_counter.v+1

$\mathrm{pH} \_$counter.v $=\mathrm{pH}$ _counter.v+1

seq $=18$

// Sequences 22-23 together with Sequence 18 build one 20 sec-unit.

\#22, base_vol_reset,5

// Base volume adjustment is every $30 \mathrm{~min}$ (at pH_counter=0)

$/ /$ and is interlaced in between two $\mathrm{pH}$ measurements (at pH_counter=45).

base_vol_0.v=base_vol_1.v

IF (base_vol_1.v<0) $\{$ base_vol_0.v=0

seq $=23$

\#23, base_vol_determination,5

// New base volume determination. 
base_vol_1.v=base_vol_0.v+8*(pH.sp-pH_value_1.v)-6*(pH_value_1.v-pH_value_0.v) base_counter.v=base_counter.v +1

$\mathrm{pH} \_$counter.v=pH_counter.v+1 seq $=18$ 

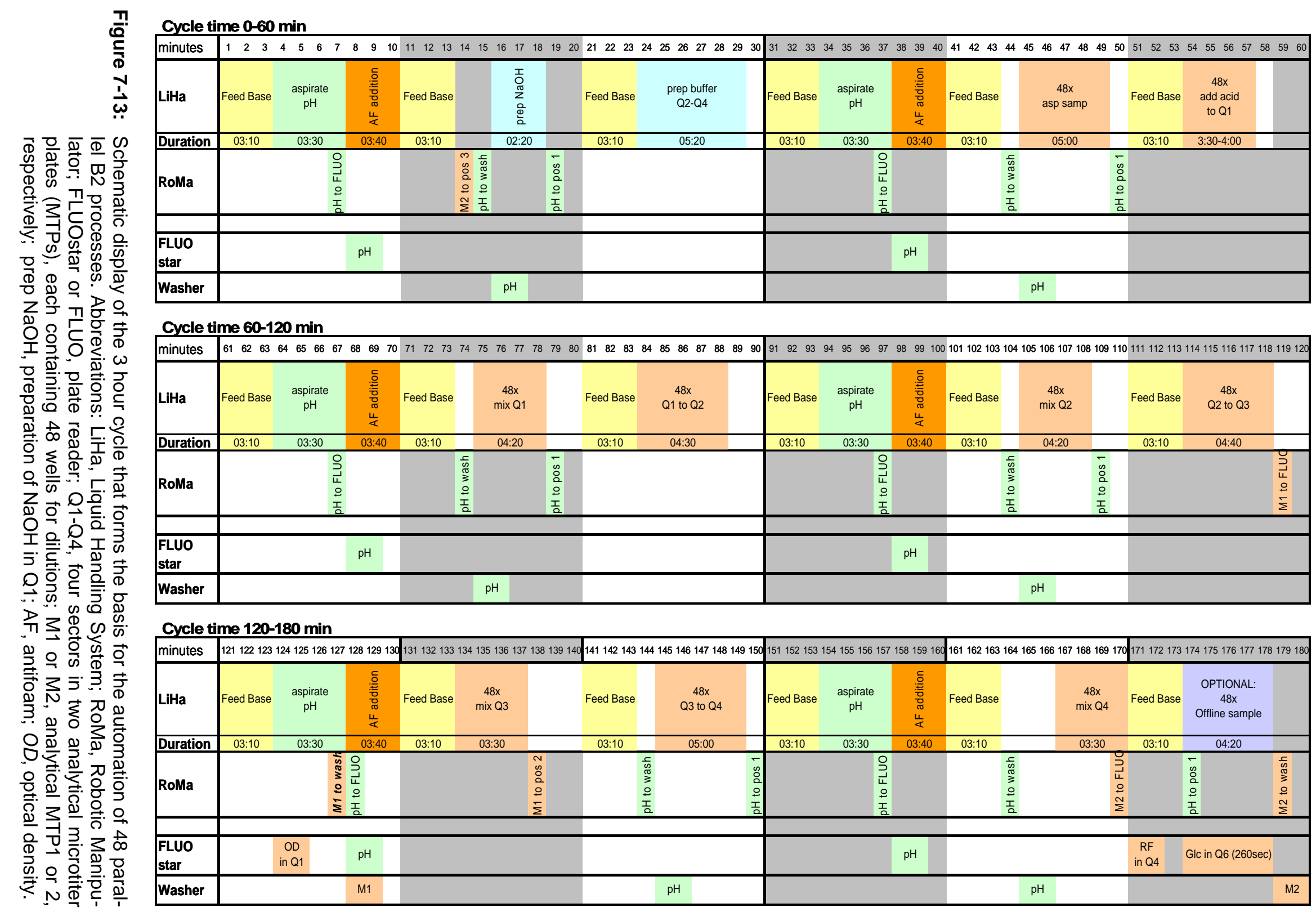

Cycle time 120-180 min

minutes $\quad 121122123124125126127128129130131132133134135136137138139140|141142143144145146147148149150| 151152153154155156157158159160|161162163164165166167168169170| 171172173174175176177178179180$

\begin{tabular}{|c|c|c|c|c|c|c|c|c|c|c|c|c|c|c|c|c|}
\hline LiHa & Feed Base & $\begin{array}{c}\text { aspirate } \\
\mathrm{pH}\end{array}$ & 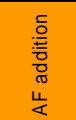 & Feed Base & $\begin{array}{c}48 x \\
\text { mix Q3 }\end{array}$ & & Feed Base & $\begin{array}{c}48 \mathrm{x} \\
\mathrm{Q} \text { to } \mathrm{Q} 4\end{array}$ & Feed Base & $\begin{array}{c}\text { aspirate } \\
\mathrm{pH}\end{array}$ & 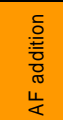 & Feed Base & $\begin{array}{c}48 x \\
\operatorname{mix} Q 4\end{array}$ & Feed Base & $\begin{array}{l}\text { OPTIONAL: } \\
48 \mathrm{x} \\
\text { Offline sample }\end{array}$ & \\
\hline Duration & $03: 10$ & 03:30 & $03: 40$ & $03: 10$ & $03: 30$ & & 03:10 & $05: 00$ & $03: 10$ & $03: 30$ & 03:40 & $03: 10$ & $03: 30$ & $03: 10$ & $04: 20$ & \\
\hline RoMa & & & & & & 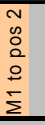 & & & 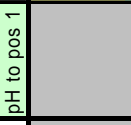 & $\begin{array}{l}\mathrm{O} \\
\mathrm{I} \\
\mathrm{u} \\
\mathrm{I} \\
\mathrm{I}\end{array}$ & & 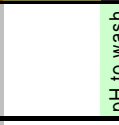 & & & 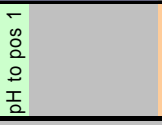 & 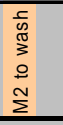 \\
\hline $\begin{array}{l}\text { FLUO } \\
\text { star }\end{array}$ & & $\begin{array}{c}\mathrm{OD} \\
\text { in } \mathrm{Q} 1\end{array}$ & $\mathrm{pH}$ & & & & & & & & $\mathrm{pH}$ & & & $\begin{array}{c}\mathrm{RF} \\
\text { in Q4 }\end{array}$ & Glc in Q6 (260sec) & \\
\hline Washer & & & M1 & & & & & pH & & & & & & & & M2 \\
\hline
\end{tabular}




\subsubsection{LabVIEW routines and GEMINI worklists integrated in GEMINI scripts}

Table 7-8: $\quad$ Software items necessary for the operation of 48-fold parallel milliliter B2 processes

\begin{tabular}{|c|c|}
\hline Name of LabView VI (*.vi) & Actions \\
\hline 48_Measure_pH.vi & Starts pH measurement in Fluostar with given test and layout and saves pH.dat and all_pH.dat (order $1,2,3)$ \\
\hline 48_init_pH.vi & Deletes pH.dat and all_pH.dat; replaces values in 48_prev_pH.dat with desired values \\
\hline 48_read_pH_write_base_worklist.vi & $\begin{array}{l}\text { Reads the measured pH values from pH.dat and generates base_worklist.gwl, base_vol.dat, } \\
\text { prev_base_vol.dat, all_base_vol.dat }\end{array}$ \\
\hline 48_read_pH7,0_write_base_worklist.vi & Same as above, but uses $\mathrm{pH}=7,0$ instead of measured $\mathrm{pH}$ for calculation of base_vol for all reactors \\
\hline 48_init_base.vi & Deletes all_base_vol.dat; replaces values in base_vol.dat with 0.5 , in prev_base_vol.dat with 0 \\
\hline 48_AF_add+wash_worklist.vi & Generates AF_worklist and AF_wash_worklist based on AF setpoint profile \\
\hline Empty_AF_worklist.vi & Generates an empty AF_worklist and AF_wash_worklist and deletes all_AF.dat \\
\hline 48 feed_worklist.vi & Writes feed_worklist via 48fold input vector (is used independently outside of Gemini) \\
\hline Empty_feed_worklist.vi & Generates an empty feed_worklist and deletes all_feed.dat \\
\hline 48_measure_glc.vi & Starts glucose measurment in Fluostar with given test and layout (pump1!) \\
\hline 48 measure_RF.vi & Starts RF measurment in Fluostar with given test and layout \\
\hline 48_measure_OD.vi & Starts OD measurment in Fluostar with given test and layout (change to light ducts for absorbance) \\
\hline motor_geminibase2.vi & $\begin{array}{l}\text { Motor movements in MTP photometer (for changes between absorbance/fluorescence); necessary to be } \\
\text { used initially before each run in order to "prime" motor properly and avoid overheating }\end{array}$ \\
\hline 48_mpw_start.vi & Starts the MTP with the wash program for analytical flat bottom MTPs \\
\hline $48 \_$mpw_pH_start.vi & Starts the MTP with the wash program for HydroPlate (round bottom MTP) \\
\hline Plateln.vi & Opens the measuring chamer of the MTP reader \\
\hline PlateOut.vi & Closes the measuring chamer of the MTP reader \\
\hline ErrorScannerRSP.vi & $\begin{array}{l}\text { Checks error status of Gemini (via pipes) on the local computer every } 5 \mathrm{sec} \text { and suppresses occuring errors; } \\
\text { if error re-occurs, it writes a "1" into the file alarm.dat which can generate an alarm action }\end{array}$ \\
\hline ErrorMonitor.vi & $\begin{array}{l}\text { Checks error status of Gemini (via pipes) from any computer over the net every } 5 \mathrm{sec} \text { (can NOT suppress } \\
\text { occuring errors, but keeps running, if LabView is blocked on the computer on which Gemini is running) }\end{array}$ \\
\hline AlarmClock.vi & Starts playing music (via Mediaplayer) upon a "1" in file alarm.dat (no actions, if status is "0") \\
\hline Data for pH control (*.dat or *.txt) & Remarks \\
\hline $\begin{array}{l}\text { 48_pH_set.txt } \\
\text { (generated by saving } 48 \_p H-\text { set.xls as a textfile) }\end{array}$ & pH setpoint profile for at least the estimated process time \\
\hline base_vol.dat & Contains the actual base volume for 48 reactors \\
\hline prev_base_vol.dat & Contains the previous base volume for 48 reactors \\
\hline prev_pH.dat & Contains the previous $\mathrm{pH}$ of 48 reactors \\
\hline $\begin{array}{l}\text { MUST NOT exist BEFORE pH meas: } \\
\text { pH.dat }\end{array}$ & $\begin{array}{l}\text { pH.dat, containing the actual } \mathrm{pH} \text { values, MUST NOT exist at the beginning of the process } \\
\text { (is always newly generated by each } \mathrm{pH} \text { measurement and deleted after update of base_vol) }\end{array}$ \\
\hline \multicolumn{2}{|l|}{ Necessary worklists (*.gwl) } \\
\hline base_worklist.gwl & Contains actual base volumes to be added to 48 reactors; is created by 48 read_pH_write_base_worklist.vi \\
\hline feed_worklist.gwl & Contains actual feed volumes; empty before start of feeding; $35.6 \mu \mathrm{L}$ for $\mathrm{VR}=10 \mathrm{~mL}$ (for glc $=397 \mathrm{~g} / \mathrm{L}$ ) \\
\hline AF_worklist.gwl & Contains actual antifoam volumes to be added to 48 reactors; is created by 48 AF_add+wash_worklist.vi \\
\hline AF_wash_worklist.gwl & Contains actual wash volumes for 48 reactors; is created by $48 \_A F \_a d d+$ wash_worklist.vi \\
\hline Variables in Gemini & Meaning/function \\
\hline $\mathrm{col}$ & Colums of reactors in X-Block ( $1=8$ reactors, $2=16$ reactors, $\ldots, 6=48$ reactors) \\
\hline Total_time & Total process time expressed in loops of $3 \mathrm{~h}^{\star}$ Offline_frequency $(24 \mathrm{~h}$ when Offline_frequency $=8$ ) \\
\hline Counter_all & For Offline_frequency $=8$ : Counter_all $=1=>$ total proces time $=24 \mathrm{~h} ; 2=>48 \mathrm{~h} ; 3=>72 \mathrm{~h}$ \\
\hline Offline_frequency & Determines at the end of which $3 \mathrm{~h}$-loop offline samples will be taken \\
\hline Offline_counter & Counts from 1 up to Offline_frequency at the end of each $3 \mathrm{~h}$-loop; is set $=0$ after offline sampling \\
\hline counter & Counts from 1 up to 3 at the end of each 10 min-loop within every 30min-loop \\
\hline Name of LabView SubVls & Actions \\
\hline Rearrange_48array_to_1,9,17.vi & rearranges elements in $48 x$ arrays from $1,2,3, \ldots 47,48$ to $1,9,17, \ldots 40,48$ (order from columns to rows) \\
\hline Rearrange_48array_to1,2,3.vi & rearranges elements in $48 x$ arrays from $1,9,17, \ldots 40,48$ to $1,2,3, \ldots 47,48$ (order from rows to columns) \\
\hline BK_Calc_processtime_sec.vi & calculates the actual time in seconds of the day and month \\
\hline Beispiel_File lesen.vi & \\
\hline
\end{tabular}




\subsubsection{Liquid Classes}

The laboratory robot (Tecan, Crailsheim) contained a feature that enabled individual settings for handling liquids with different fluid properties. These settings were saved in individual liquid classes (LCs). Specific liquid classes were established for all fluids used during automated B2 processes in the milliliter setup. They are summarized in Table 7-9, before the individual settings of each liquid class are listed on the following pages. The liquid class "Water 3-15 $\mu \mathrm{L}$ " was not used but is provided for comparison, as it comprises the default settings of the manufacturer.

Table 7-9: $\quad$ Liquid classes (LCs) for automated B2 processes in the milliliter setup.

\begin{tabular}{|c|c|c|c|}
\hline Name of LC & Appropriate fluids & Pipetting mode & Volumetric range \\
\hline BK_sampling & Sample for at-line analysis & single & $5-30 \mu \mathrm{L}$ \\
\hline BK_pHsample & Sample for at-line $\mathrm{pH}$ & single & $5-30 \mu \mathrm{L}$ \\
\hline BK_sampling_offline & Sample for offline analysis & single & $5-30 \mu \mathrm{L}$ \\
\hline BK_VD1 & 1x diluted sample (Q1-Q2) & single & $5-30 \mu \mathrm{L}$ \\
\hline BK_transfer & $3^{\text {rd }}$ and $4^{\text {th }}$ dilution step & single & $5-50 \mu \mathrm{L}$ \\
\hline BK_mix & All mixing steps in Q1-Q4 & single & $15-500 \mu \mathrm{L}$ \\
\hline BK_buffer_prep & $\begin{array}{l}\text { Aquatic solutions (buffer, } \mathrm{NaOH} \text {, } \\
\text { citric acid, } 2-3 x \text { diluted sample) }\end{array}$ & single & all volumes \\
\hline BK_AF_wash & Acetone & single & all volumes \\
\hline Water (Tecan default) & Aquatic solutions & & 3-15 $\mu \mathrm{L}$ \\
\hline BK_feed_50\% & Feed ( $397 \mathrm{~g} \mathrm{~L}^{-1}$ glucose) & multi & $1-500 \mu \mathrm{L}$ \\
\hline BK_NH4OH & $12.5 \% \mathrm{NH}_{4} \mathrm{OH}$ & multi & $\begin{array}{c}0.5-3 \mu \mathrm{L} \\
3-20 \mu \mathrm{L} \\
20-500 \mu \mathrm{L}\end{array}$ \\
\hline BK_AF & $\begin{array}{c}\text { Antifoam Biospumex } 30 \mathrm{~K} \\
\text { (diluted } 1: 4 \text { with water) }\end{array}$ & multi & all volumes \\
\hline
\end{tabular}


BK sampling : Std. \& Low Vol. $<5-30 \mu l>$

Global

Detection Mode Liquid Conductivity Detection speed

Double Distance $4 \mathrm{~mm}$

Liquid Detection

detect simultaneously and twice with all tips good

$60 \mathrm{~mm} / \mathrm{s}$

Clot Detection

Detection speed $50 \mathrm{~mm} / \mathrm{s}$

clot Limit $4 \mathrm{~mm}$

Aspirate

$$
\begin{array}{r}
\text { Aspiration Speed } \\
\text { Delay } \\
\text { System Trailing Airgap } \\
\text { Leading Airgap } \\
\text { Speed } \\
\text { Delay } \\
\text { Trailing Airgap } \\
\text { Speed } \\
\text { Delay } \\
\text { Excess Volume } \\
\text { Replace after } \\
\text { Conditioning Volume }
\end{array}
$$$$
\text { Use Pinch Valve }
$$$$
\text { Use Liquid Detection }
$$$$
\text { Aspiration Position }
$$$$
\text { Use Clot Detection }
$$

Mix before Aspiration

Retract Tips to

Retract speed

Dispense

Dispense speed Breakoff speed

Delay

Trailing Airgap after each Dispense

Use Pinch Valve

Use Liquid Detection

$$
\text { Dispense Position }
$$

Tip Touching

Mix after Dispense

Retract Tips to

Retract speed

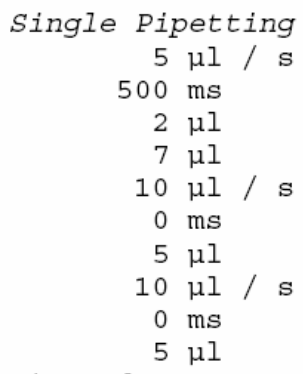

$2 \mu 1$

7 l

$10 \mu l / s$

$0 \mathrm{~ms}$

$5 \mu 1$

$10 \mu \mathrm{l} / \mathrm{s}$

$0 \mathrm{~ms}$

$5 \mu l$

Discard to waste

$1 \mathrm{x}$ Discard

$10 \mu 1$

Back to vessel

yes
Multi Pipetting
$10 \mu \mathrm{l} / \mathrm{s}$
$400 \mathrm{~ms}$
$5 \mu l$
$0 \mu \mathrm{l}$
$10 \mu \mathrm{l} / \mathrm{s}$
$0 \mathrm{~ms}$
$0 \mu \mathrm{l}$
$10 \mu l / \mathrm{s}$
$0 \mathrm{~ms}$
$15 \mu l$

Discard to waste

$2 \times$ Discard

$0.25 \mu l$

Back to vessel yes

no

$z-\max \pm$ offset, no tracking $0 \mathrm{~mm}$

$X$ : center

no

no

$\mathrm{z}$-dispense $0 \mathrm{~mm}$

$20 \mathrm{~mm} / \mathrm{s}$

$$
\begin{gathered}
\text { Single pipetting } \\
10 \mu \mathrm{\mu l} / \mathrm{s} \\
30 \mu \mathrm{\mu l} / \mathrm{s} \\
0 \mathrm{~ms} \\
\text { no } \\
\text { yes }
\end{gathered}
$$

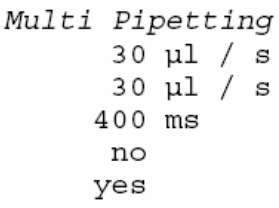

yes

no $\mathrm{z}$-dispense \pm offset, no tracking $-4 \mathrm{~mm}$ $\mathrm{X}$ : center

no tip touching

no

$z$-travel $0 \mathrm{~mm}$

$50 \mathrm{~mm} / \mathrm{s}$ 
BK_sampling_offline : Std. \& Low Vol. $<5-30 \mu l>$

Global

Detection Mode Liquid Conductivity Detection speed Double Distance

Detection speed clot Limit

\section{Aspirate}

$$
\begin{aligned}
& \text { Aspiration speed } \\
& \text { Delay } \\
& \text { System Trailing Airgap } \\
& \text { Leading Airgap } \\
& \text { Speed } \\
& \text { Delay } \\
& \text { Trailing Airgap } \\
& \text { speed } \\
& \text { Delay } \\
& \text { Excess Volume } \\
& \text { Replace after }
\end{aligned}
$$$$
\text { Conditioning Volume }
$$

Use Pinch Valve

Use Liquid Detection Aspiration Position

Use Clot Detection

Mix before Aspiration

Retract Tips to Retract speed

\section{Dispense}

$$
\begin{array}{r}
\text { Dispense } \\
\text { Breakoff } \\
\text { Dpeed } \\
\text { Delay }
\end{array}
$$

Trailing Airgap after each Dispense Use Pinch Valve Use Liquid Detection
Dispense Position

Tip Touching

Mix after Dispense

Retract Tips to
Retract Speed
Liquid Detection

detect simultaneously and twice with all tips good

$60 \mathrm{~mm} / \mathrm{s}$

$4 \mathrm{~mm}$

Clot Detection

$50 \mathrm{~mm} / \mathrm{s}$

$4 \mathrm{~mm}$

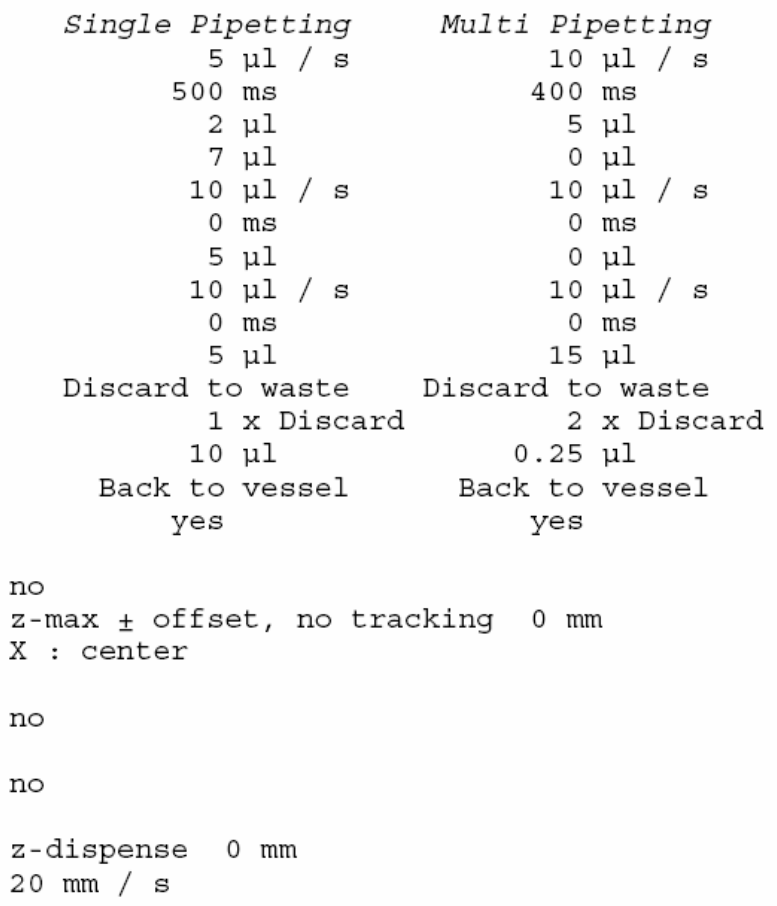

Calibration
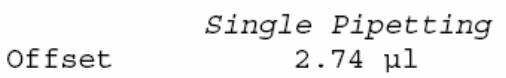
BK_transfer : Std. \& Low Vol. <5 - 50 ul >

Global

$\underline{\text { Aspirate }}$

$$
\begin{aligned}
& \text { Aspiration Speed } \\
& \text { Delay } \\
& \text { System Trailing Airgap } \\
& \text { Leading Airgap } \\
& \text { Speed } \\
& \text { Delay } \\
& \text { Trailing Airgap } \\
& \text { speed } \\
& \text { Delay } \\
& \text { Excess Volume } \\
& \text { Replace after }
\end{aligned}
$$$$
\text { Conditioning Volume }
$$

Use Pinch Valve

Use Liquid Detection

Aspiration Position

Use Clot Detection

Mix before Aspiration

Retract Tips to Retract Speed
Liquid Detection

detect simultaneously and twice with all tips good

$60 \mathrm{~mm} / \mathrm{s}$

$4 \mathrm{~mm}$

Clot Detection

$50 \mathrm{~mm} / \mathrm{s}$

$4 \mathrm{~mm}$

Dispense

Dispense
Breakoff
Speed
Delay

Trailing Airgap after each Dispense
Use Pinch Valve

$\begin{array}{rr}\text { Single Pipetting } & \text { Multi Pipetting } \\ 5 \mu \mathrm{\mu l} / \mathrm{s} & 45 \mu \mathrm{s} / \mathrm{s} \\ 400 \mathrm{~ms} & 500 \mathrm{~ms} \\ 5 \mu l & 7 \mu l \\ 10 \mu l & 0 \mu l \\ 10 \mu l / \mathrm{s} & 10 \mu \mathrm{s} / \mathrm{s} \\ 0 \mathrm{~ms} & 0 \mathrm{~ms} \\ 5 \mu l & 0 \mu l \\ 10 \mu l / \mathrm{s} & 10 \mu \mathrm{s} / \mathrm{s} \\ 0 \mathrm{~ms} & 0 \mathrm{~ms} \\ 10 \mu l & 30 \mu l\end{array}$

Discard to waste Discard to waste

$1 \mathrm{x}$ Discard $2 \mathrm{x}$ Discard

$10 \mu 1$

$1 \mu l$

Back to vessel Back to vessel yes yes

no

$z-\max \pm$ offset, no tracking $0 \mathrm{~mm}$

$\mathrm{X}$ : center

no

no

$\mathrm{z}$-dispense $\quad-10 \mathrm{~mm}$

$20 \mathrm{~mm} / \mathrm{s}$

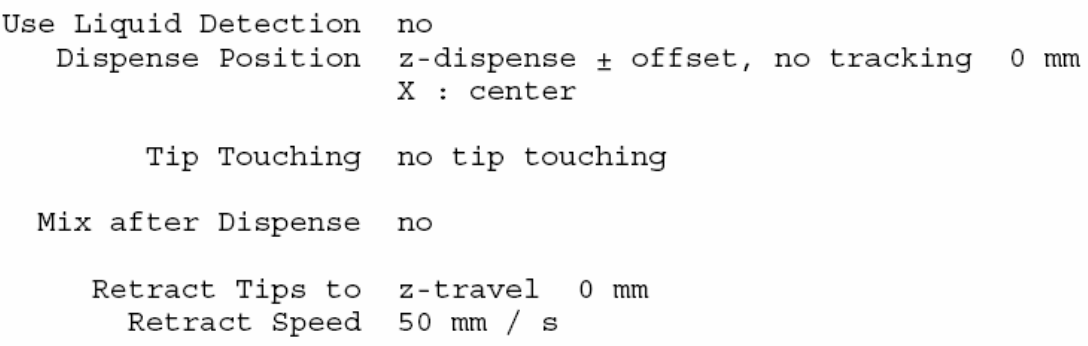

Offiset Factor

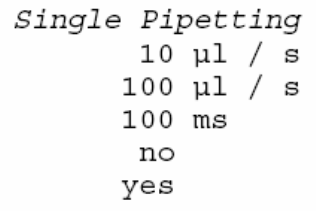


BK_mix : Std. \& Low Vol. <15 - 500 ll>

Global

Liquid Detection

Detection Mode detect simultaneously and twice with all tips

Liquid Conductivity good

Detection speed $60 \mathrm{~mm} / \mathrm{s}$

Double Distance $4 \mathrm{~mm}$

Detection speed $50 \mathrm{~mm} / \mathrm{s}$

Clot Limit $4 \mathrm{~mm}$

\section{Aspirate}

$$
\begin{aligned}
& \text { Aspiration Speed } \\
& \text { Delay } \\
& \text { System Trailing Airgap } \\
& \text { Leading Airgap } \\
& \text { Speed } \\
& \text { Delay } \\
& \text { Trailing Airgap } \\
& \text { Speed } \\
& \text { Delay } \\
& \text { Excess Volume } \\
& \text { Replace after } \\
& \text { Conditioning Volume }
\end{aligned}
$$

Use Pinch Valve

Use Liquid Detection Aspiration Position

Use Clot Detection no

Mix before Aspiration

Retract Tips to Retract speed

\section{$\underline{\text { Dispense }}$}

Dispense Speed Breakoff speed Delay

Trailing Airgap after each Dispense Use Pinch Valve

Use Liquid Detection Dispense Position

Tip Touching

Mix after Dispense

Retract Tips to $\mathrm{z}$-travel $0 \mathrm{~mm}$ Retract speed $50 \mathrm{~mm} / \mathrm{s}$

no

$\mathrm{X}$ : center

no tip touching

no$$
\text { (n) }
$$

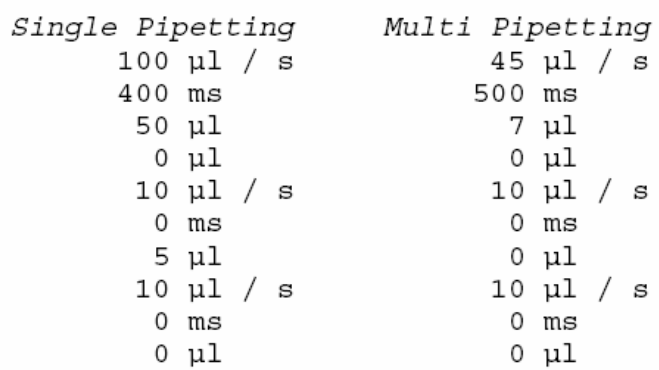

Multi Pipetting

$110 \mu \mathrm{l} / \mathrm{s}$

$110 \mu \mathrm{l} / \mathrm{s}$

$400 \mathrm{~ms}$

yes

yes

$z$-max \pm offset, no tracking $0 \mathrm{~mm}$

\section{Calibration}

Offset 
BK_buffer_prep : Std. \& Low Vol. <all volumes >

Global

Detection Mode Liquid Conductivity

Detection speed

Double Distance

Detection speed

clot Limit

\section{Aspirate}

$$
\begin{array}{r}
\text { Aspiration Speed } \\
\text { Delay } \\
\text { System Trailing Airgap } \\
\text { Leading Airgap } \\
\text { Speed } \\
\text { Delay } \\
\text { Trailing Airgap } \\
\text { Speed } \\
\text { Delay } \\
\text { Excess Volume } \\
\text { Replace after } \\
\text { Conditioning Volume } \\
\text { Use Pinch Valve }
\end{array}
$$

Use Liquid Detection

Aspiration Position

Use Clot Detection

Mix before Aspiration

Retract Tips to Retract speed

\section{Dispense}

Dispense Speed

Breakoff speed

Delay

Trailing Airgap after each Dispense

Use Pinch Valve

Use Liquid Detection Dispense Position

Tip Touching

Mix after Dispense

Retract Tips to

Retract speed
Liquid Detection

detect simultaneously and twice with all tips good

$60 \mathrm{~mm} / \mathrm{s}$

$4 \mathrm{~mm}$

Clot Detection

$50 \mathrm{~mm} / \mathrm{s}$

$4 \mathrm{~mm}$

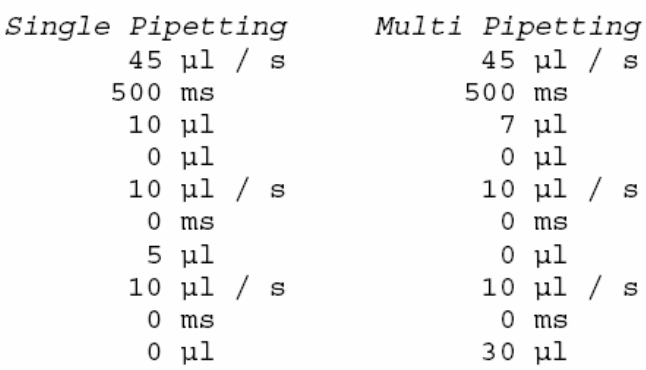

Multi Pipetting

$45 \mu l / s$

$500 \mathrm{~ms}$

$7 \mu 1$

$0 \mu l$

$10 \mu \mathrm{l} / \mathrm{s}$

$0 \mathrm{~ms}$

$0 \mu l$

$10 \mu l / s$

$0 \mathrm{~ms}$

$30 \mu 1$

$1 \times$ Discard $2 \times$ Discard yes

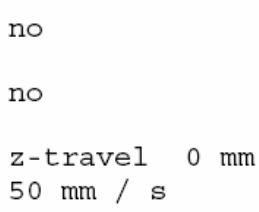

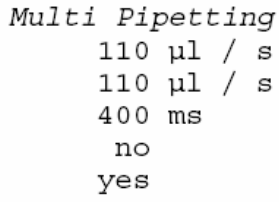

no

$z$-max \pm offset, no tracking $0 \mathrm{~mm}$ $\mathrm{X}$ : center

no tip touching

no

z-travel $0 \mathrm{~mm}$

$50 \mathrm{~mm} / \mathrm{s}$

\section{Calibration}

$$
\text { Single Pipetting }
$$$$
10.1 \mu l
$$$$
1
$$

Multi Pipetting

$0 \mu 1$ 
BK_AF_wash : Std. \& Low Vol. <all volumes>

Global

Detection Mode Liquid Conductivity Detection speed Double Distance

Detection speed clot Limit

\section{$\underline{\text { Aspirate }}$}

$$
\begin{array}{r}
\text { Aspiration Speed } \\
\text { Delay } \\
\text { Trailing Airgap } \\
\text { Leading Airgap } \\
\text { Speed } \\
\text { Delay } \\
\text { Trailing Airgap } \\
\text { Speed } \\
\text { Delay } \\
\text { Excess Volume } \\
\text { Replace after } \\
\text { Conditioning Volume }
\end{array}
$$$$
\text { Use Liquid Detection }
$$$$
\text { Aspiration Position }
$$$$
\text { Use Clot Detection }
$$$$
\text { Mix before Aspiration }
$$

Retract Tips to Retract speed

\section{Dispense}

Dispense Speed Delay

Trailing Airgap after each Dispense Use Pinch Valve

Use Liquid Detection Dispense Position

Tip Touching

Mix after Dispense

Retract Tips to Retract speed
Liquid Detection

detect with odd / even tips twice good

$60 \mathrm{~mm} / \mathrm{s}$

$4 \mathrm{~mm}$

Clot Detection

$50 \mathrm{~mm} / \mathrm{s}$

$4 \mathrm{~mm}$ Breakoff speed

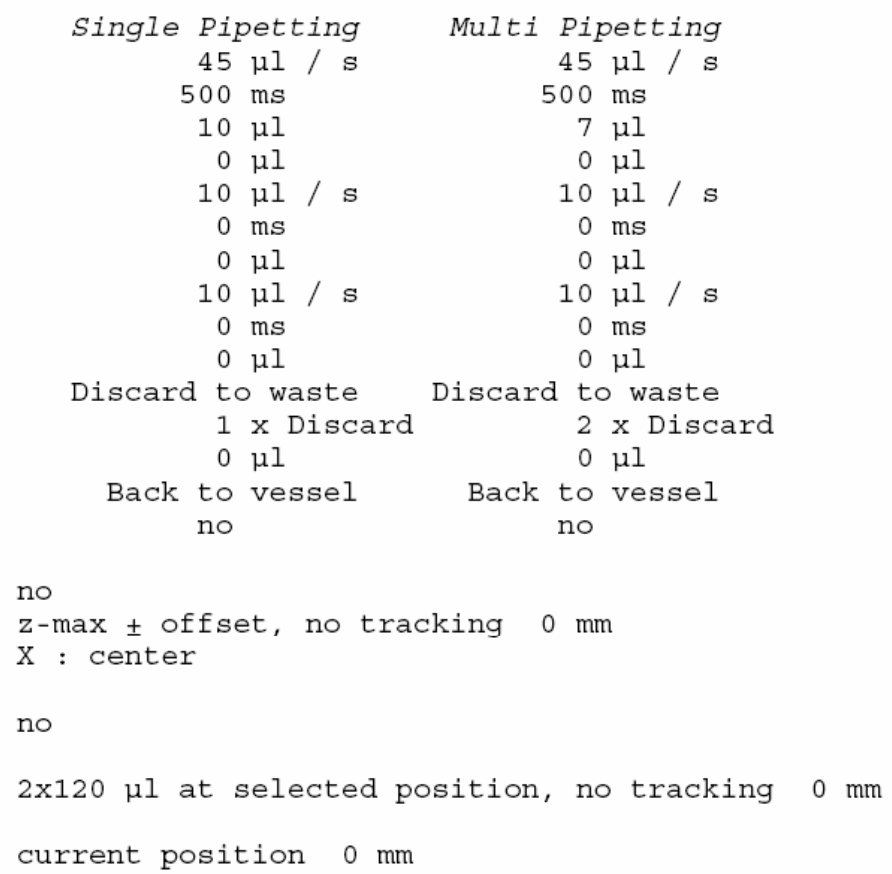

\section{Calibration}

Offset Factor 
Water : Std. \& Low Vol. $<3-15 \mu l>$

Global

$$
\begin{array}{r}
\text { Detection Mode } \\
\text { Liquid Conductivity } \\
\text { Detection Speed } \\
\text { Double Distance } \\
\text { Detection Speed } \\
\text { Clot Limit }
\end{array}
$$

\section{Aspirate}

$$
\begin{aligned}
& \text { Aspiration Speed } \\
& \text { Delay } \\
& \text { System Trailing Airgap } \\
& \text { Leading Airgap } \\
& \text { speed } \\
& \text { Trailing Airgap } \\
& \text { Speed } \\
& \text { Delay } \\
& \text { Excess Volume }
\end{aligned}
$$

Use Liquid Detection Aspiration Position

On Detection Error

Use Clot Detection

Mix before Aspiration Retract Tips to
Retract Speed

\section{Dispense}

$$
\begin{array}{r}
\text { Dispense } \\
\text { Breakoff } \\
\text { Speed } \\
\text { Delay }
\end{array}
$$

\begin{tabular}{|c|c|c|c|}
\hline Single Pip & petting & Multi Pi & ipettins \\
\hline 10 & $\mu l / \mathrm{s}$ & 10 & $0 \mu l$ / \\
\hline 400 & $\mathrm{~ms}$ & 400 & $0 \mathrm{~ms}$ \\
\hline 2 & $\mu 1$ & & $5 \mu l$ \\
\hline 7 & $\mu l$ & & $0 \mu l$ \\
\hline 10 & $\mu l / \mathrm{s}$ & 10 & $0 \mu l /$ \\
\hline 0 & $\mathrm{~ms}$ & & $0 \mathrm{~ms}$ \\
\hline 5 & $\mu l$ & & $0 \mu l$ \\
\hline 10 & $\mu l / \mathrm{s}$ & 10 & $0 \mu l /$ \\
\hline 0 & $\mathrm{~ms}$ & & $0 \mathrm{~ms}$ \\
\hline 0 & $\mu l$ & 15 & $5 \mu l$ \\
\hline
\end{tabular}

Trailing Airgap after each Dispense Use Pinch Valve

$$
\begin{gathered}
\text { Use Liquid Detection } \\
\text { Dispense Position }
\end{gathered}
$$

Tip Touching

Mix after Dispense

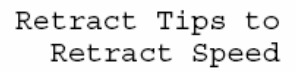

\section{Calibration}

Offset

Factor
Liquid Detection

detect with odd / even tips twice good

$60 \mathrm{~mm} / \mathrm{s}$

$4 \mathrm{~mm}$

Clot Detection

$50 \mathrm{~mm} / \mathrm{s}$

$4 \mathrm{~mm}$
Discard to waste

Discard to waste
$1 \mathrm{x}$ Discard
$2 \times$ Discard

$0 \mu 1$

Back to vessel

no

$0.25 \mu l$

Back to vessel yes

yes

liquid level \pm offset, with tracking $1 \mathrm{~mm}$ $\mathrm{X}$ : center

user prompt

no

no

liquid level $-5 \mathrm{~mm}$

$20 \mathrm{~mm} / \mathrm{s}$

no

$\mathrm{z}$-dispense \pm offset, no tracking $0 \mathrm{~mm}$

$\mathrm{X}$ : center

no tip touching

no

$\mathrm{z}$-dispense $0 \mathrm{~mm}$

$50 \mathrm{~mm} / \mathrm{s}$

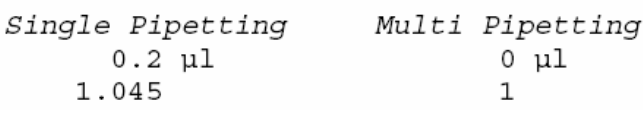

$30 \mu l / \mathrm{s}$

$400 \mathrm{~ms}$

yes 
BK_feed_50\% : Std. \& Low Vol. <1 - 500ul>

Global

Aspirate

Aspiration Speed
Delay
System Trailing Airgap
Leading Airgap
Speed
Delay
Trailing Airgap
Speed
Delay
Excess Volume
Replace after
Conditioning Volume

Use Pinch Valve

Use Liquid Detection Aspiration Position

Use Clot Detection no

Mix before Aspiration

Retract Tips to

Retract Speed $20 \mathrm{~mm} / \mathrm{s}$

\section{Dispense}

Dispense Speed Breakoff speed

Trailing Airgap after each Dispense Use Pinch Valve

Use Liquid Detection Dispense Position

Tip Touching

Mix after Dispense

Retract Tips to $\mathrm{z}$-travel $0 \mathrm{~mm}$ Retract speed $50 \mathrm{~mm} / \mathrm{s}$

no

$\mathrm{X}$ : center

no tip touching

no

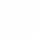

Liquid Detection

detect simultaneously and twice with all tips good

$60 \mathrm{~mm} / \mathrm{s}$

$4 \mathrm{~mm}$

Clot Detection

$50 \mathrm{~mm} / \mathrm{s}$

$4 \mathrm{~mm}$

$\begin{array}{rr}\text { Single Pipetting } & \text { Multi Pipetting } \\ 10 \mu l / \mathrm{s} & 10 \mu \mathrm{s} / \mathrm{s} \\ 500 \mathrm{~ms} & 400 \mathrm{~ms} \\ 2 \mu l & 5 \mu l \\ 7 \mu l & 0 \mu \mathrm{\mu l} \\ 10 \mu l / \mathrm{s} & 10 \mu \mathrm{s} / \mathrm{s} \\ 0 \mathrm{~ms} & 0 \mathrm{~ms} \\ 5 \mu l & 0 \mu \mathrm{s} \\ 10 \mu l / \mathrm{s} & 10 \mu l / \mathrm{s} \\ 0 \mathrm{~ms} & 0 \mathrm{~ms} \\ 10 \mu l & 15 \mu l\end{array}$

Discard to waste

Discard to waste

$0.25 \mu l$

$0.25 \mu l$

Back to vessel Back to vessel

yes

yes

no

$z$-max \pm offset, no tracking $0 \mathrm{~mm}$

$\mathrm{X}$ : center

no

-dispense $-5 \mathrm{~mm}$
Multi Pipetting

$30 \mu l / s$

$30 \mu \mathrm{l} / \mathrm{s}$

$400 \mathrm{~ms}$

no

yes

$\mathrm{z}$-dispense \pm offset, no tracking $0 \mathrm{~mm}$

\section{Calibration}

Offset

Single Pipetting

$2.6 \mu 1$

1.01

Multi Pipetting

$0 \mu l$

Factor$$
\text { 1.01 }
$$

1 
BK_NH4OH : Std. \& Low Vol. $<3-20 \mu l>$

Global

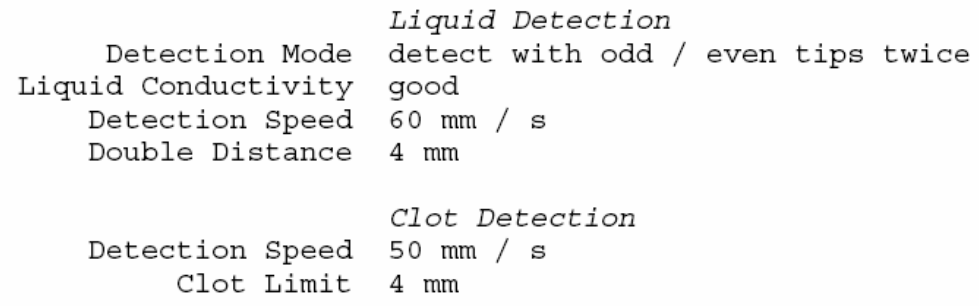

\section{Aspirate}

$$
\begin{aligned}
& \text { Aspiration Speed } \\
& \text { Delay } \\
& \text { System Trailing Airgap } \\
& \text { Leading Airgap } \\
& \text { Speed } \\
& \text { Delay } \\
& \text { Trailing Airgap } \\
& \text { Speed } \\
& \text { Delay }
\end{aligned}
$$
Conditioning Volume

Use Pinch Valve

Use Liquid Detection Aspiration Position

Use Clot Detection

Mix before Aspiration

Retract Tips to Retract Speed

\section{Dispense}

Dispense speed Breakoff speed Delay

Trailing Airgap after each Dispense Use Pinch Valve

Use Liquid Detection Dispense Position

Tip Touching

Mix after Dispense

Retract Tips to Retract speed

\section{Calibration}

Offset Factor

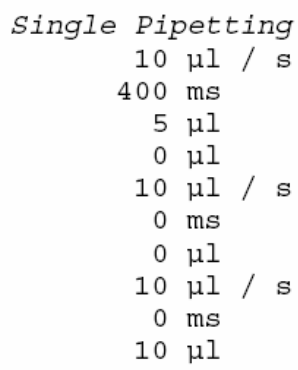

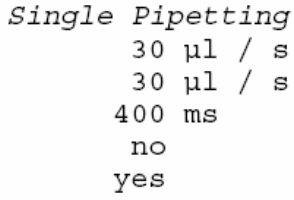


BK_NH4OH : Std. \& Low Vol. $<20-500 \mu l>$

Global

$\begin{aligned} & \text { Liquid Detection } \\ \text { Detection Mode } & \text { detect with odd / even tips twice } \\ \text { Liquid Conductivity } & \text { good } \\ \text { Detection Speed } & 60 \mathrm{~mm} / \mathrm{s} \\ \text { Double Distance } & 4 \mathrm{~mm} \\ & \text { Clot Detection } \\ & 50 \mathrm{~mm} / \mathrm{s} \\ \text { Detection Speed } & 4 \mathrm{~mm} \\ \text { Clot Limit } & 4 \mathrm{~mm}\end{aligned}$

\section{Aspirate}

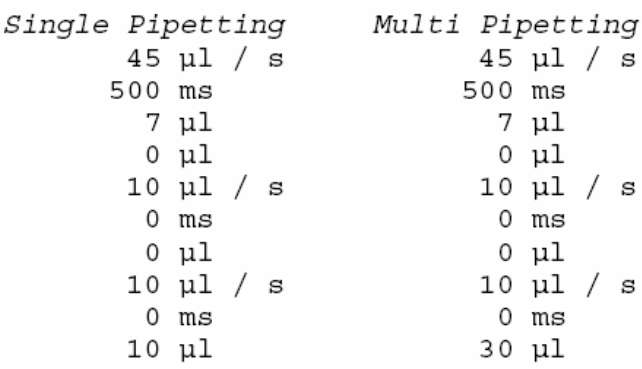

\section{Dispense}

Dispense Speed Breakoff speed Delay Trailing Airgap after each Dispense Use Pinch Valve

Use Liquid Detection Dispense Position

Tip Touching

Mix after Dispense

Retract Tips to Retract Speed

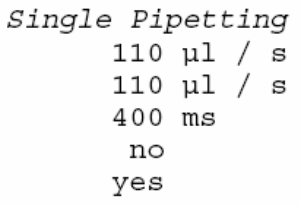

\section{Calibration}

Single Pipetting

$-0.062 \mu \mathrm{I}$

1.008

Multi Pipetting

$-0.062 \mu \mathrm{l}$

1.008 
BK_AF : Std. \& Low Vol. <all volumes>

Global

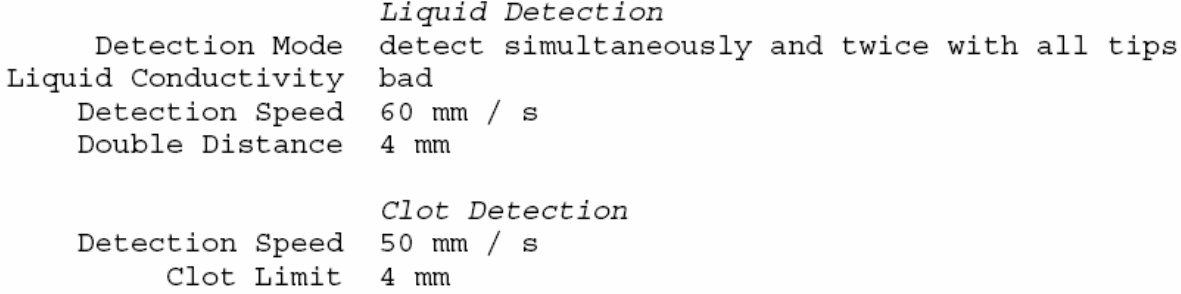

\section{Aspirate}

\begin{tabular}{|c|c|c|c|}
\hline Single $P i$ & petting & Mult $i$ & petting \\
\hline 10 & $\mu l / \mathrm{s}$ & 10 & $\mu l / \mathrm{s}$ \\
\hline 3000 & $\mathrm{~ms}$ & 400 & $\mathrm{~ms}$ \\
\hline 5 & $\mu l$ & 5 & $\mu l$ \\
\hline 0 & $\mu 1$ & 0 & $\mu l$ \\
\hline 10 & $\mu \mathrm{l} / \mathrm{s}$ & 10 & $\mu l / \mathrm{s}$ \\
\hline 0 & $\mathrm{~ms}$ & 0 & $\mathrm{~ms}$ \\
\hline 0 & $\mu l$ & 0 & $\mu l$ \\
\hline 10 & $\mu l / \mathrm{s}$ & 10 & $\mu l$ / \\
\hline 0 & $\mathrm{~ms}$ & 0 & $\mathrm{~ms}$ \\
\hline 0 & $\mu l$ & 0 & $\mu l$ \\
\hline
\end{tabular}

Discard to waste Discard to waste $1 \mathrm{x}$ Discard $1 \mathrm{x}$ Discard

$0 \mu l \quad 0.25 \mu l$

Back to vessel Back to vessel

no no

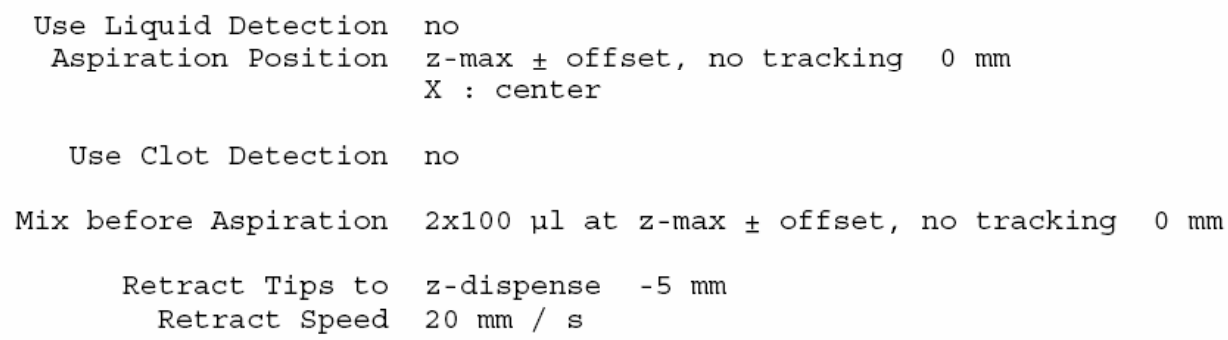

$\underline{\text { Dispense }}$

$$
\begin{array}{r}
\text { Dispense Speed } \\
\text { Breakoff Speed } \\
\text { Delay }
\end{array}
$$

Trailing Airgap after each Dispense

Use Pinch Valve

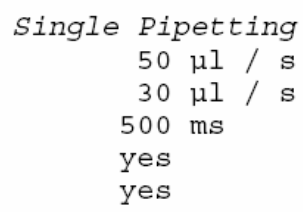

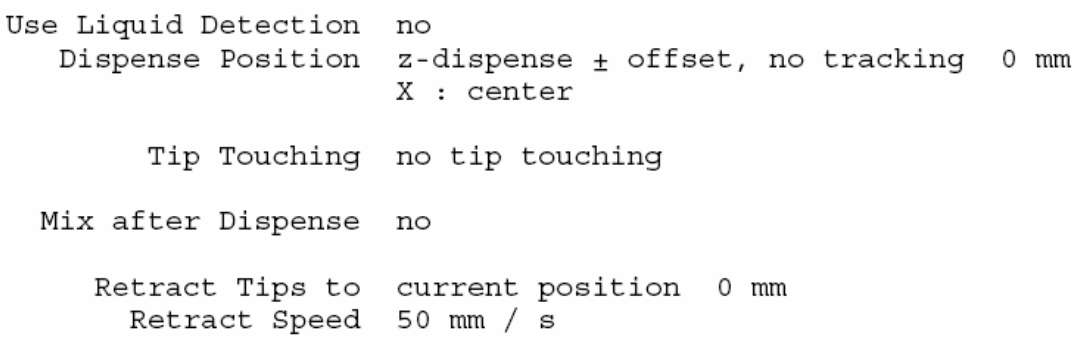


